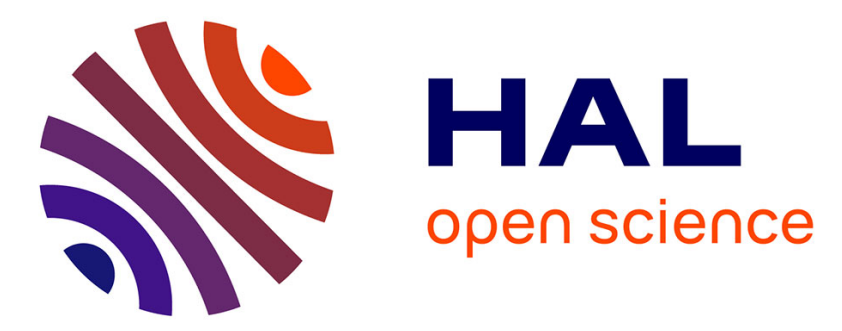

\title{
Exploring Bacterial Colonies in Solid Foods or Model Foods Using Non-Destructive Techniques
}

\author{
Anne Thierry, Sophie Jeanson, Sylvie Lortal
}

\section{To cite this version:}

Anne Thierry, Sophie Jeanson, Sylvie Lortal (Dir.). Exploring Bacterial Colonies in Solid Foods or Model Foods Using Non-Destructive Techniques. Frontiers in Microbiology, Février 2016, 103 p., 2016, Frontiers in Microbiology, 978-2-88919-743-9. 10.3389/978-2-88919-743-9 . hal-01276165

\section{HAL Id: hal-01276165 \\ https://hal.science/hal-01276165}

Submitted on 5 Jun 2020

HAL is a multi-disciplinary open access archive for the deposit and dissemination of scientific research documents, whether they are published or not. The documents may come from teaching and research institutions in France or abroad, or from public or private research centers.
L'archive ouverte pluridisciplinaire HAL, est destinée au dépôt et à la diffusion de documents scientifiques de niveau recherche, publiés ou non, émanant des établissements d'enseignement et de recherche français ou étrangers, des laboratoires publics ou privés. 


\section{(x) \\ EXPLORING BACTERIAL COLONIES IN SOLID FOODS OR MODEL FOODS USING NON-DESTRUGTIVE TECHNIQUES}

EDITED BY: Sophie Jeanson, Sylvie Lortal and Anne Thierry PUBLISHED IN: Frontiers in Microbiology

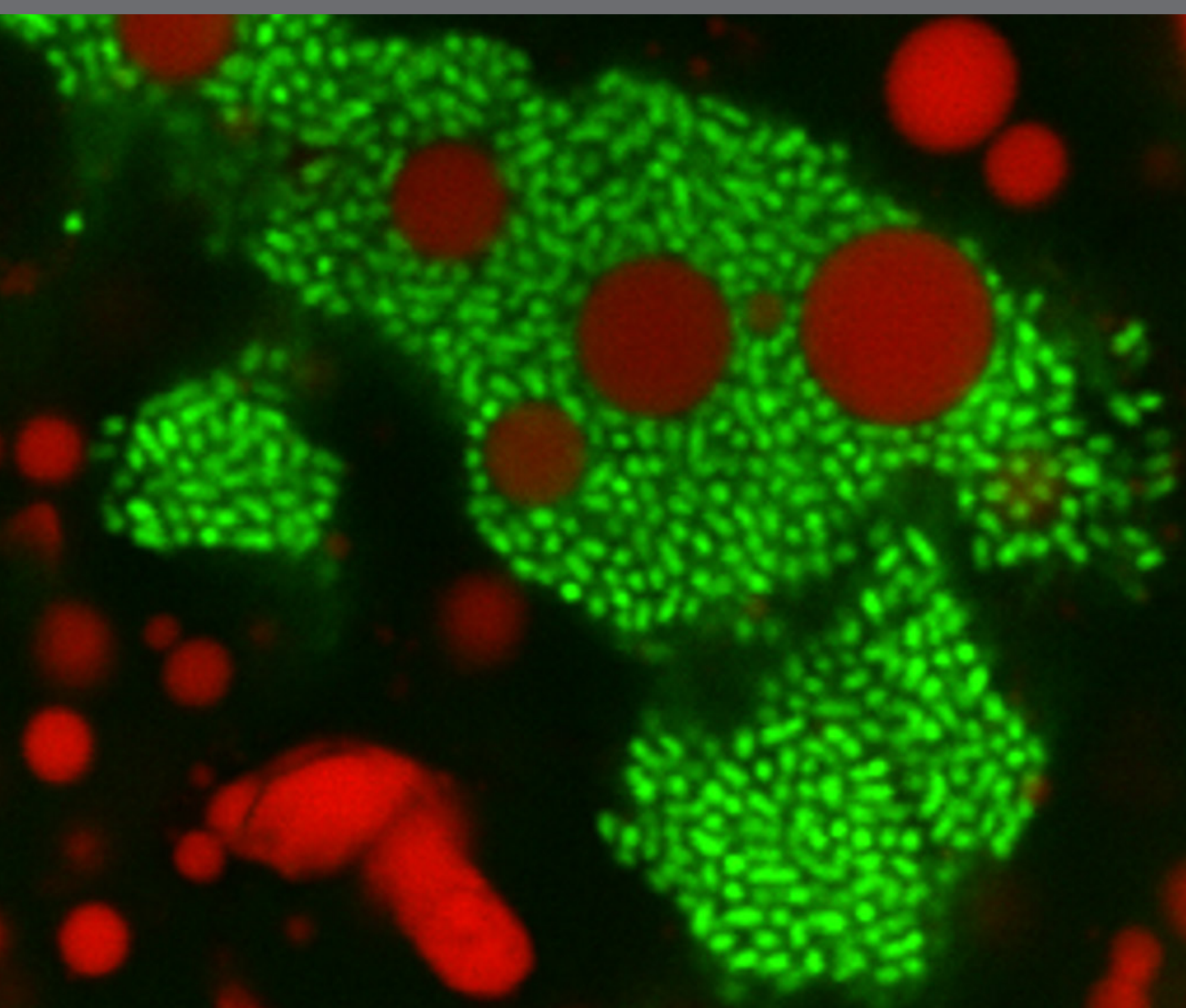




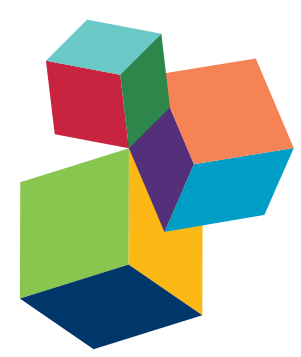

Frontiers Copyright Statement

(c) Copyright 2007-2016 Frontiers Media SA. All rights reserved.

All content included on this site, such as text, graphics, logos, button

icons, images, video/audio clips,

downloads, data compilations and

software, is the property of or is

licensed to Frontiers Media SA

("Frontiers") or its licensees and/or subcontractors. The copyright in the text of individual articles is the property of their respective authors, subject to a license granted to Frontiers.

The compilation of articles constituting

this e-book, wherever published,

as well as the compilation of all other

content on this site, is the exclusive property of Frontiers. For the conditions for downloading and copying of e-books from Frontiers' website, please see the Terms for Website Use. If purchasing Frontiers e-books from other websites or sources, the conditions of the website concerned apply.

Images and graphics not forming part of user-contributed materials may not be downloaded or copied without permission.

Individual articles may be downloaded and reproduced in accordance with the principles of the CC-BY licence subject to any copyright or other notices. They may not be re-sold as an e-book.

As author or other contributor you grant a $C C-B Y$ licence to others to reproduce your articles, including any graphics and third-party materials supplied by you, in accordance with the Conditions for Website Use and subject to any copyright notices which you include in connection with your articles and materials.

All copyright, and all rights therein, are protected by national and international copyright laws.

The above represents a summary only. For the full conditions see the Conditions for Authors and the Conditions for Website Use.

ISSN 1664-8714 ISBN 978-2-88919-743-9

DOI 10.3389/978-2-88919-743-9

\section{About Frontiers}

Frontiers is more than just an open-access publisher of scholarly articles: it is a pioneering approach to the world of academia, radically improving the way scholarly research is managed. The grand vision of Frontiers is a world where all people have an equal opportunity to seek, share and generate knowledge. Frontiers provides immediate and permanent online open access to all its publications, but this alone is not enough to realize our grand goals.

\section{Frontiers Journal Series}

The Frontiers Journal Series is a multi-tier and interdisciplinary set of open-access, online journals, promising a paradigm shift from the current review, selection and dissemination processes in academic publishing. All Frontiers journals are driven by researchers for researchers; therefore, they constitute a service to the scholarly community. At the same time, the Frontiers Journal Series operates on a revolutionary invention, the tiered publishing system, initially addressing specific communities of scholars, and gradually climbing up to broader public understanding, thus serving the interests of the lay society, too.

\section{Dedication to Quality}

Each Frontiers article is a landmark of the highest quality, thanks to genuinely collaborative interactions between authors and review editors, who include some of the world's best academicians. Research must be certified by peers before entering a stream of knowledge that may eventually reach the public - and shape society; therefore, Frontiers only applies the most rigorous and unbiased reviews.

Frontiers revolutionizes research publishing by freely delivering the most outstanding research, evaluated with no bias from both the academic and social point of view. By applying the most advanced information technologies, Frontiers is catapulting scholarly publishing into a new generation.

\section{What are Frontiers Research Topics?}

Frontiers Research Topics are very popular trademarks of the Frontiers Journals Series: they are collections of at least ten articles, all centered on a particular subject. With their unique mix of varied contributions from Original Research to Review Articles, Frontiers Research Topics unify the most influential researchers, the latest key findings and historical advances in a hot research area! Find out more on how to host your own Frontiers Research Topic or contribute to one as an author by contacting the Frontiers Editorial Office: researchtopics@frontiersin.org 


\section{EXPLORING BACTERIAL COLONIES IN SOLID FOODS OR MODEL FOODS USING NON-DESTRUCTIVE TECHNIQUES}

Topic Editors:

Sophie Jeanson, French National Institute for Agricultural Research (INRA), UMR1253, France

Sylvie Lortal, French National Institute for Agricultural Research (INRA), UMR1253, France Anne Thierry, French National Institute for Agricultural Research (INRA), UMR1253, France

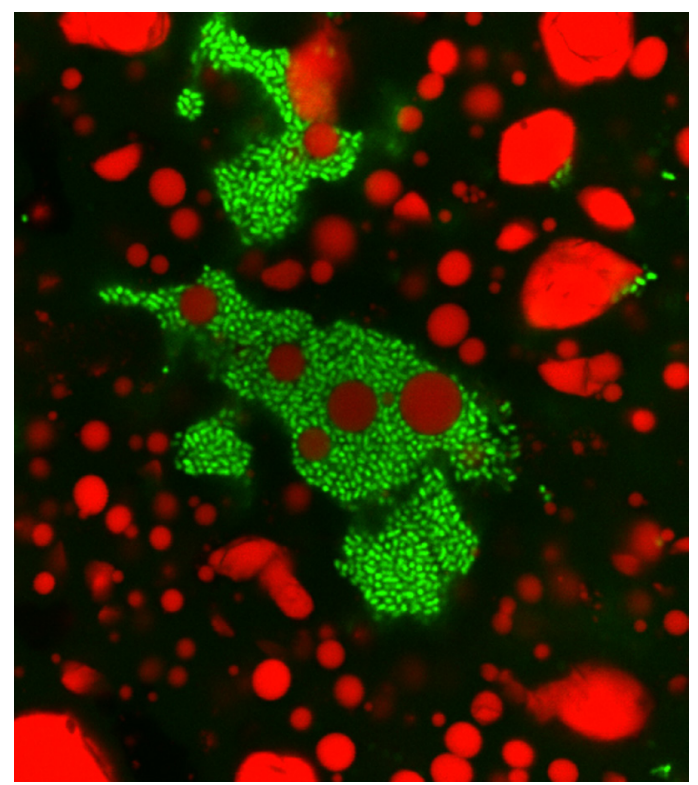

Colony of lactic acid bacteria (Lactococcus lactis) in cheese, surrounded by fat-protein network, observed by confocal laser-scanning microscopy. Bacteria are shown in green, protein matrix in black, lipids in red. Image by Marie-Noëlle Madec, INRA, STLO, France

Bacteria are always present in foods, either as initial contamination or as technological agents. In solid foods, they are immobilized and develop as colonies. So far, there is a lack of knowkedge about the bacteria in colonies, growth and physiology. Non-destructive and resolute techniques, such as fluorescent microscopy, now allow investigating the world of bacteria in colonies and their surroundings in food, at the microscopic scale.

Citation: Jeanson, S., Lortal, S., Thierry, A., eds. (2016). Exploring Bacterial Colonies in Solid Foods or Model Foods Using Non-Destructive Techniques. Lausanne: Frontiers Media. doi: 10.3389/978-2-88919-743-9 


\section{Table of Contents}

04 Editorial: Exploring Bacterial Colonies in Solid Foods or Model Foods Using Non-Destructive Techniques

Sophie Jeanson and Anne Thierry

06 Bacterial Colonies in Solid Media and Foods: A Review on Their Growth and Interactions with the Micro-Environment

Sophie Jeanson, Juliane Floury, Valérie Gagnaire, Sylvie Lortal and Anne Thierry

26 Colonial vs. planktonic type of growth: mathematical modeling of microbial dynamics on surfaces and in liquid, semi-liquid and solid foods

Panagiotis N. Skandamis and Sophie Jeanson

35 Recent trends in non-invasive in situ techniques to monitor bacterial colonies in solid (model) food

María M. Lobete, Estefania Noriega Fernandez and Jan F. M. Van Impe

44 Growth and location of bacterial colonies within dairy foods using microscopy techniques: a review

Cian D. Hickey, Jeremiah J. Sheehan, Martin G. Wilkinson and Mark A. E. Auty

52 In situ examination of Lactobacillus brevis after exposure to an oxidizing disinfectant

Yu Zhao, Susanne Knøchel and Henrik Siegumfeldt

60 Laser-induced speckle scatter patterns in Bacillus colonies

Huisung Kim, Atul K. Singh, Arun K. Bhunia and Euiwon Bae

69 Microcalorimetric study of the growth of Streptococcus thermophilus in renneted milk

Irina Stulova, Natalja Kabanova, Tiina Kriščiunaite, Kaarel Adamberg, Tiiu-Maie Laht and Raivo Vilu

82 Measurement of $\mathrm{pH}$ micro-heterogeneity in natural cheese matrices by fluorescence lifetime imaging

Zuzana Burdikova, Zdenek Svindrych, Jan Pala, Cian D. Hickey, Martin G. Wilkinson, Jiri Panek, Mark A. E. Auty, Ammasi Periasamy and Jeremiah J. Sheehan

92 Diffusion of solutes inside bacterial colonies immobilized in model cheese depends on their physicochemical properties: a time-lapse microscopy study Juliane Floury, Ilham El Mourdi, Juliana V. C. Silva, Sylvie Lortal, Anne Thierry and Sophie Jeanson 


\title{
Editorial: Exploring Bacterial Colonies in Solid Foods or Model Foods Using Non-Destructive Techniques
}

\author{
Sophie Jeanson ${ }^{1,2 *}$ and Anne Thierry ${ }^{1,2}$ \\ ${ }^{1}$ Institut National de la Recherche Agronomique, UMR1253, Science and Technology of Milk and Eggs, Rennes, France, \\ ${ }^{2}$ AGROCAMPUS OUEST, UMR1253, Science and Technology of Milk and Eggs, Rennes, France
}

Keywords: bacterial colonies, growth, physiology, solid foods, modeling, non-destructive techniques

The Editorial on the Research Topic

Exploring Bacterial Colonies in Solid Foods or Model Foods Using Non-Destructive Techniques

Bacteria are present in all foods, whether they are indigenous or inoculated. They can be beneficial to the quality of foods, responsible for food spoilage, or even pathogens. In solid food products, bacteria are immobilized. They thus grow as colonies entrapped within the food products or on the food surfaces. In both cases, bacteria interact with the solid matrix, sometimes facing difficulties to access the nutrients as nutrients have to diffuse from the matrix to the bacterial colonies. Bacterial development can thus be impaired in solid matrices in comparison to planktonic growth. To control the growth of bacteria in solid foods is then of major importance. In the case of pathogens, it is crucial for safety issues to predict how the bacteria present as initial contaminants will develop. In the case of inoculated bacteria, such as lactic acid bacteria, it is also crucial to control their development because they are responsible for the final quality of the food products. However, studies on the growth of bacteria have been essentially focused on growth in liquid media. Resolute techniques, which include both microcopy approaches and quantitative techniques, have now been developed to observe colonies at the microscopic scale. They allow studying the variation of growth in different contexts either in model growth culture media or in model foods.

This Research Topic starts with four review papers. A first comprehensive review about bacterial colonies redraws the history of the studies on colonies, synthetizes the conditions of growth in which growth in colonies differs from planktonic growth, and finally presents concepts of the interaction of bacterial colonies with the food matrix in which they grow, with cheese as an example (Jeanson et al.). The second review demonstrates the importance of modeling the growth of bacterial colonies in solids foods despite a lack of knowledge. The different types of models and their potential consequences on decisions are presented and discussed (Skandamis and Jeanson). The third review shows how non-invasive techniques can be used to observe bacterial growth and also quantify the bacterial metabolism in colonies at the microscopic scale (Lobete et al.). The last review paper shows how microscopy techniques are particularly valuable to increase knowledge about bacterial colonies in model foods and foods. Fluorescent microscopy allows targeting metabolites and understand the physiology of bacteria within colonies (Hickey et al.).

The five following papers present original results in the field. Using an automated microscope coupled with fluorescence dyes, it was possible to demonstrate that the exposure to an oxidizing disinfectant led to different morphologies of cells depending on the strains and that dead cells were randomly distributed within the micro-colonies (Zhao et al.). The following study demonstrates the 
accuracy of a phenotyping technology based on laser-induced speckle scatter patterns in Bacillus colonies. The authors showed that the distribution of speckle size is modified during the growth of colonies (Kim et al.).

The three following studies investigate bacterial colonies of lactic acid bacteria in their food environment in model cheeses or investigate the $\mathrm{pH}$ in their surrounding micro-environment in Cheddar cheeses. Physiology and growth of Streptococcus thermophilus colonies was compared in liquid milk and renneted milk using microcalorimetry. The results showed that above a threshold size of colonies (low inoculation levels), growth rates and metabolism in renneted milk differed from the one observed in liquid milk (Stulova et al.). The use of fluorescence lifetime imaging was shown to be relevant to investigate the $\mathrm{pH}$ microheterogeneity in Cheddar cheeses (Burdikova et al.). These results suggest that the surroundings of bacterial colonies within cheeses could differ from homogeneous growth conditions in liquid medium. The last study questioned the access of bacteria in colonies to their nutrients, through the assessment of diffusion of solutes inside colonies. The diffusion of high molecular weight molecules was assessed inside Lactococcus lactis colonies grown in a model cheese. The results led to the conclusion that diffusion of molecules inside bacterial colonies depends on the physicochemical properties of the molecules (Floury et al.).
In conclusion, since bacteria mainly grow in colonies in foods, increase knowledge on growth and physiology of bacteria growing as colonies is now a crucial issue. This is achieved by the recent development of non-invasive techniques that allows investigating at the microscopic scale with time lapse and quantitative analyses. Knowledge about the growth and the adaptive response of bacteria to the food environment will continue to grow by addressing the remaining questions about interactions between bacterial colonies and their food environment.

\section{AUTHOR CONTRIBUTIONS}

SJ: wrote the editorial. AT: revised and improved the editorial.

Conflict of Interest Statement: The authors declare that the research was conducted in the absence of any commercial or financial relationships that could be construed as a potential conflict of interest.

Copyright $\odot 2015$ Jeanson and Thierry. This is an open-access article distributed under the terms of the Creative Commons Attribution License (CC BY). The use, distribution or reproduction in other forums is permitted, provided the original author(s) or licensor are credited and that the original publication in this journal is cited, in accordance with accepted academic practice. No use, distribution or reproduction is permitted which does not comply with these terms. 


\section{Bacterial Colonies in Solid Media and Foods: A Review on Their Growth and Interactions with the Micro-Environment}

OPEN ACCESS

Edited by:

Jean-Christophe Augustin, Ecole Nationale Vétérinaire d'Alfort,

France

\section{Reviewed by: Ilkin Yucel Sengun, \\ Ege University, Turkey Kostas Koutsoumanis, Aristotle University of Thessaloniki, \\ Greece \\ *Correspondence: \\ Sophie Jeanson \\ sophie.jeanson@rennes.inra.fr}

Specialty section:

This article was submitted to

Food Microbiology,

a section of the journal

Frontiers in Microbiology

Received: 03 September 2015 Accepted: 31 October 2015 Published: 01 December 2015

Citation:

Jeanson S, Floury J, Gagnaire V Lortal S and Thierry A (2015) Bacterial Colonies in Solid Media and Foods: A Review on Their Growth and Interactions with the Micro-Environment. Front. Microbiol. 6:1284. doi: 10.3389/fmicb.2015.01284

\author{
Sophie Jeanson ${ }^{1,2 *}$, Juliane Floury ${ }^{1,2}$, Valérie Gagnaire ${ }^{1,2}$, Sylvie Lortal ${ }^{1,2}$ and \\ Anne Thierry ${ }^{1,2}$ \\ ${ }^{1}$ INRA, UMR1253, Science and Technology of Milk and Eggs, Rennes, France, ${ }^{2}$ AGROCAMPUS OUEST, UMR1253, \\ Science and Technology of Milk and Eggs, Rennes, France
}

Bacteria, either indigenous or added, are immobilized in solid foods where they grow as colonies. Since the 80's, relatively few research groups have explored the implications of bacteria growing as colonies and mostly focused on pathogens in large colonies on agar/gelatine media. It is only recently that high resolution imaging techniques and biophysical characterization techniques increased the understanding of the growth of bacterial colonies, for different sizes of colonies, at the microscopic level and even down to the molecular level. This review covers the studies on bacterial colony growth in agar or gelatine media mimicking the food environment and in model cheese. The following conclusions have been brought to light. Firstly, under unfavorable conditions, mimicking food conditions, the immobilization of bacteria always constrains their growth in comparison with planktonic growth and increases the sensibility of bacteria to environmental stresses. Secondly, the spatial distribution describes both the distance between colonies and the size of the colonies as a function of the initial level of population. By studying the literature, we concluded that there systematically exists a threshold that distinguishes micro-colonies (radius $<100-200 \mu \mathrm{m}$ ) from macro-colonies (radius $>200 \mu \mathrm{m})$. Micro-colonies growth resembles planktonic growth and no $\mathrm{pH}$ microgradients could be observed. Macro-colonies growth is slower than planktonic growth and $\mathrm{pH}$ microgradients could be observed in and around them due to diffusion limitations which occur around, but also inside the macro-colonies. Diffusion limitations of milk proteins have been demonstrated in a model cheese around and in the bacterial colonies. In conclusion, the impact of immobilization is predominant for macro-colonies in comparison with micro-colonies. However, the interaction between the colonies and the food matrix itself remains to be further investigated at the microscopic scale.

Keywords: bacterial colony, spatial distribution, diffusion limitation, porosity, cheese, Growth 


\section{INTRODUCTION}

Bacteria in food products, whether those added as inocula or those naturally present, are always immobilized. They develop as colonies, either on the surface of or embedded within the food matrices and interact with their micro-environment (Hickey et al., 2015). As previously stated (Hills, 2001), the bacterial cells of the colony "consume the nutrients from the surrounding (food) matrix and in return, liberates end-products into the surrounding matrix modifying its micro-environment."

Bacterial colonies and biofilms are both formed by clusters of bacteria. Whilst published research focusing on biofilms is abundant (Flemming and Wingender, 2010), that based on the bacterial colony is relatively scarce, especially with respect to food. The question remains unanswered whether there are different phenotypes of bacteria making up biofilms and colonies (and especially surface colonies). A biofilm is well-defined as: "a microbiologically derived sessile community characterized by cells that are irreversibly attached to a substratum or interface or to each other, are embedded in a matrix of extracellular polymeric substances that they have produced, and exhibit an altered phenotype with respect to growth rate and gene expression" (Donlan and Costerton, 2002). On the other hand, bacterial colonies are not so well-defined. In this review, a bacterial colony is taken as a clonal group of cells developed either on the surface of or embedded within a gel-type solid (culture medium or food) from which it takes its growth substrates. Unlike biofilms, a colony is limited by size displaying a definite maximum radius ranging between a few $\mu \mathrm{m}$ to a few $\mathrm{mm}$. As the production of extracellular polymeric substances has never been investigated in colonies, we considered not to be a mandatory property. This review will focus only on bacterial colonies and exclude biofilms.

The literature on growth and metabolism of bacteria growing in colonies is scarce. Usually, growth and metabolism of food bacteria (whether desirable or undesirable) are studied in broth media, i.e., in planktonic cultures. However, in order to predict the growth of bacteria in food, it is preferable to perform the study in conditions that closely reflect the natural condition, i.e., in solid model foods. Furthermore, it has been shown that the predictive models of growth built from data taken from liquid cultures are not accurate in describing immobilized growth, especially under stressful conditions that exist in a food medium (Pipe and Grimson, 2008; Skandamis and Jeanson, 2015). Although, the context of most studies cited in this review relates to food, all of them were performed using laboratory media, such as agar or gelatine media, mimicking the growth parameters of food ( $a_{\mathrm{w}}, \mathrm{pH}, \mathrm{NaCl}$ concentration, etc.). It is only very recently that model foods, such as a model cheese, have been used to study the growth of bacterial colonies in situ. Both agar/gelatine and foods are matrices in which bacterial colonies can be embedded (submerged colonies), or on which bacterial colonies can attach (surface colonies). However, there is a major difference between agar/gelatine based media and food matrices. Agar/gelatine media are "neutral" matrices because agar and gelatine are not themselves modified by bacteria, whilst food matrices constitute both a structure and a bound substrate for the bacteria. For example, caseins in cheese are a gel-type structure and also provide nitrogen sources to bacteria. This means that food matrices may change by the bacterial activity.

The aim of this review is to describe the growth of colonies by pointing out when and how it differs from the planktonic growth. We particularly discuss the occurrence of variability at the microscopic scale of the physiological states inside the colony, of $\mathrm{pH}$ inside and around colonies and of oxygen around the colonies. The diffusion of substrates within the matrix and the access of bacteria to the substrates is also a major concern for the bacterial activity. The second objective is to build concepts on the different situations when growth of bacteria is impacted by the growth in colonies or not, depending on the initial level of population and two other concepts on the different ways of interacting with a food matrix, i.e., "bubble" or "sponge" concepts. Finally, experimental exploration of these two concepts will be examined in model cheese. Furthermore, a large table assembles the main parameters of growth and size of colonies for different experimental culture conditions studied with several bacterial species (Table 1).

\section{HISTORICAL PERSPECTIVE OF THE SCIENTIFIC COMMUNITY WORKING ON BACTERIAL COLONIES}

As early as the 60 's, Pirt, of the University of London, had started to take into account the immobilization of bacteria in the predictive growth models (Pirt, 1967). More recently the 90's, Wimpenny, from the University of Wales, started to study the consequences for bacteria by growing as colonies. Wimpenny et al. (1995), Thomas and Wimpenny (1996b), and McKay et al. (1997) performed studies on pathogenic bacteria, mostly as large colonies $(>500 \mu \mathrm{m})$, either surface or submerged, on an agar medium. They determined several characteristics of the behavior in colonies comparing with planktonic growth, such as growth rates under different conditions, and $\mathrm{pH}$ gradients within and around colonies of different sizes. Before the research on this topic stopped at the University of Wales, Wimpenny collaborated with Brocklehurst (Walker et al., 1997; Wilson et al., 2002) of the Institute of Food Research (Norwich, UK) who was also working on the immobilized growth of pathogenic bacteria. Brocklehurst and his group (Parker et al., 1998; Wright et al., 2000; Meldrum et al., 2003) developed and patented the Gel Cassette System (Brocklehurst et al., 1995). This system has become the ideal tool to study submerged colonies in gelatine and agar media, which was associated with a nondestructive and in situ microscopic examination. It comprises a $2 \mathrm{~mm}$ thick frame in a PVC sleeve shown to be permeable to gas. The inoculated medium solidifies inside the frame and the immobilized cells develop as colonies within the formed solid gel. Subsequently, Brocklehurst collaborated with Malakar (Wageningen University, Netherlands) who worked on $\mathrm{pH}$ microgradients, introducing imaging techniques (Malakar et al., 2000), and on interactions between colonies of lactic acid bacteria (Malakar et al., 2003) and at a later date with Van Impe (Leuven University, Belgium) whose group still works at improving predictive growth models for immobilized pathogenic 


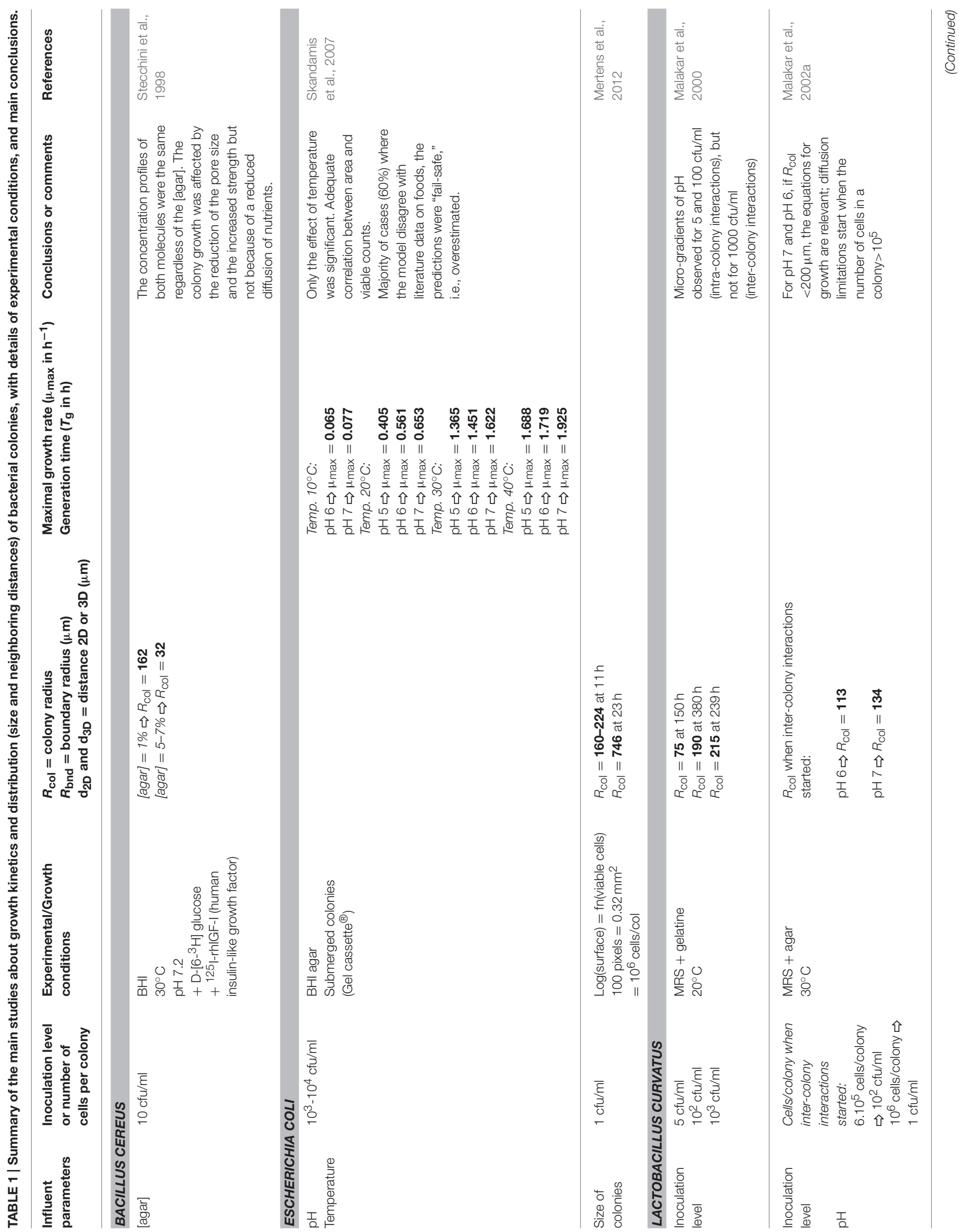




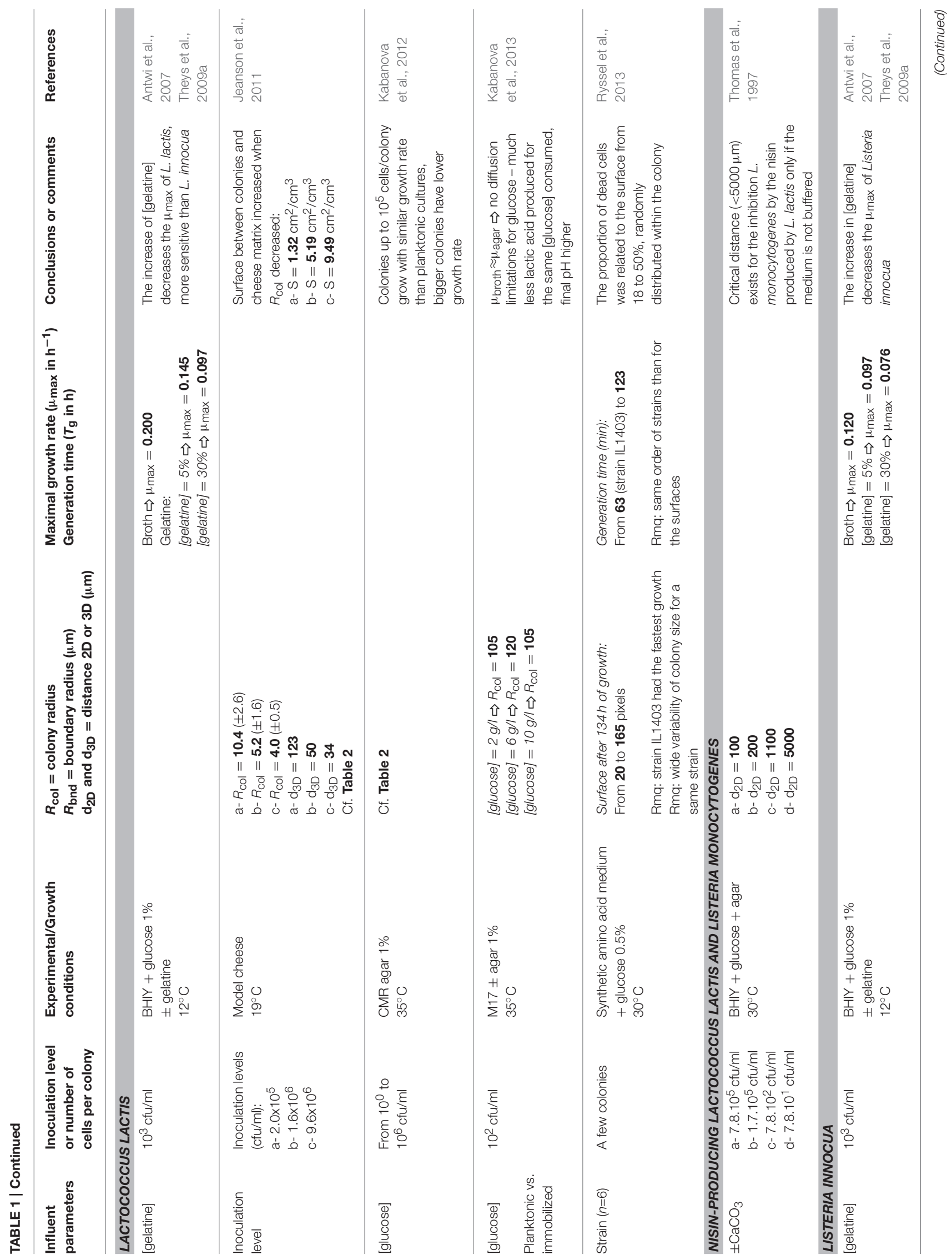




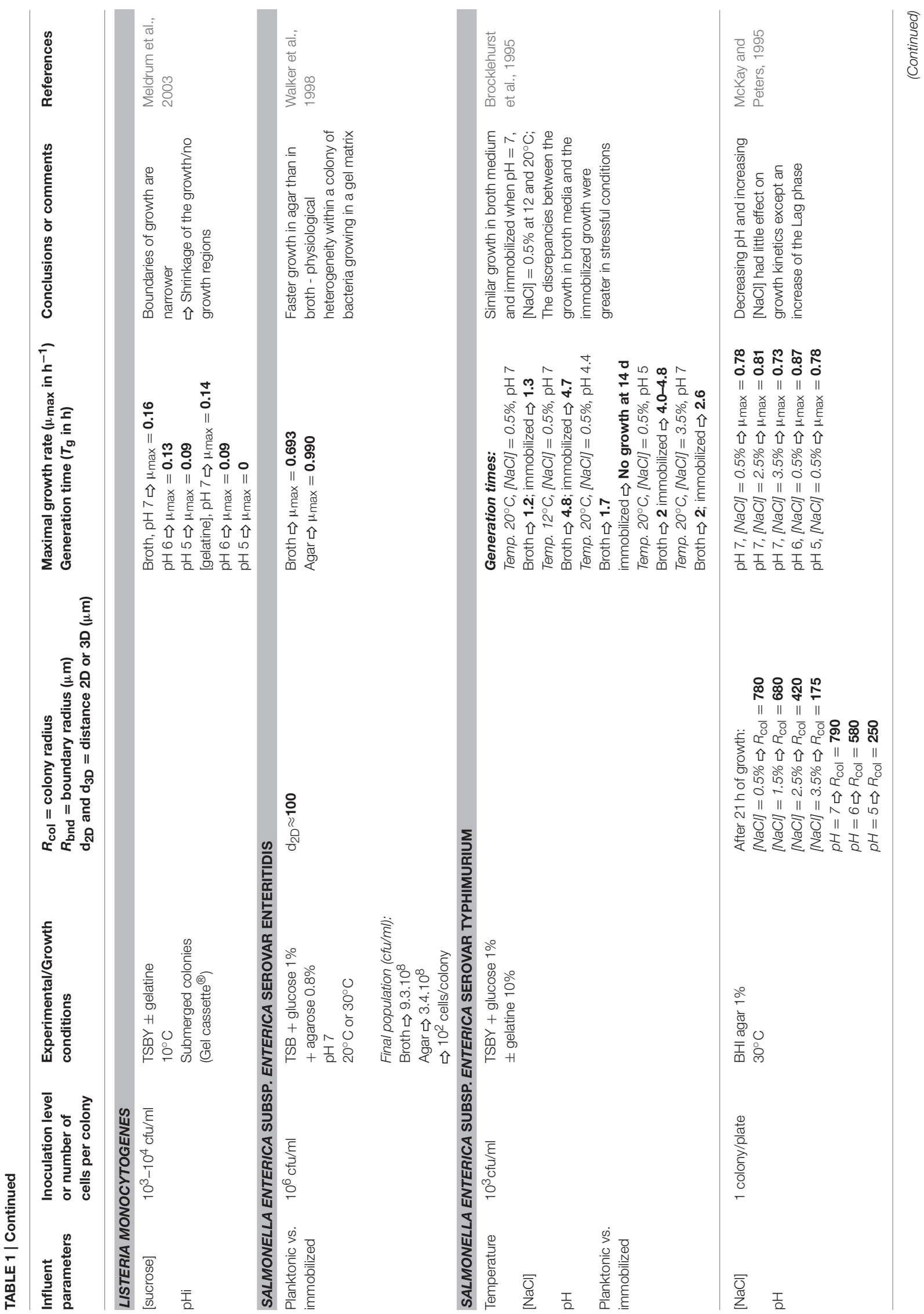




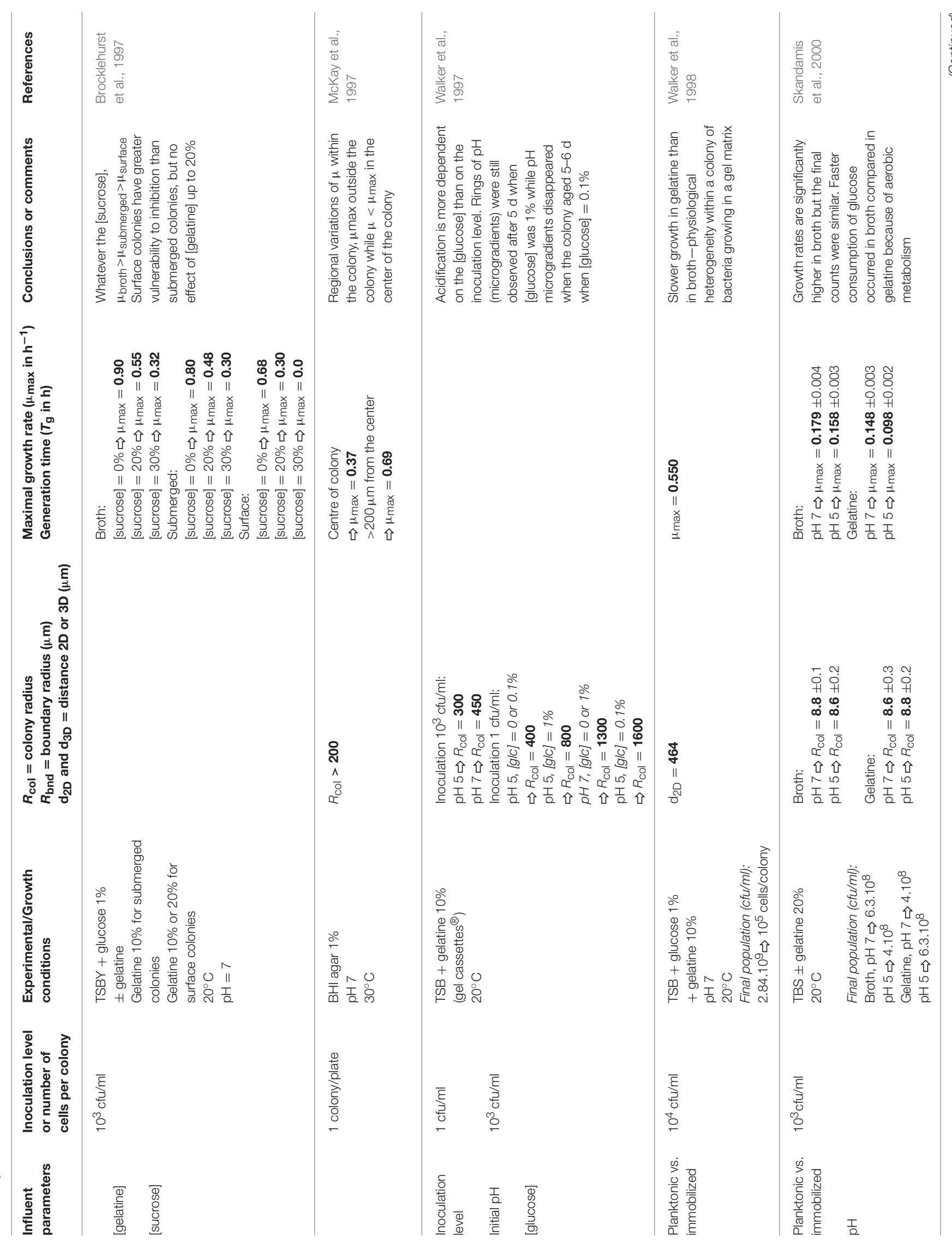




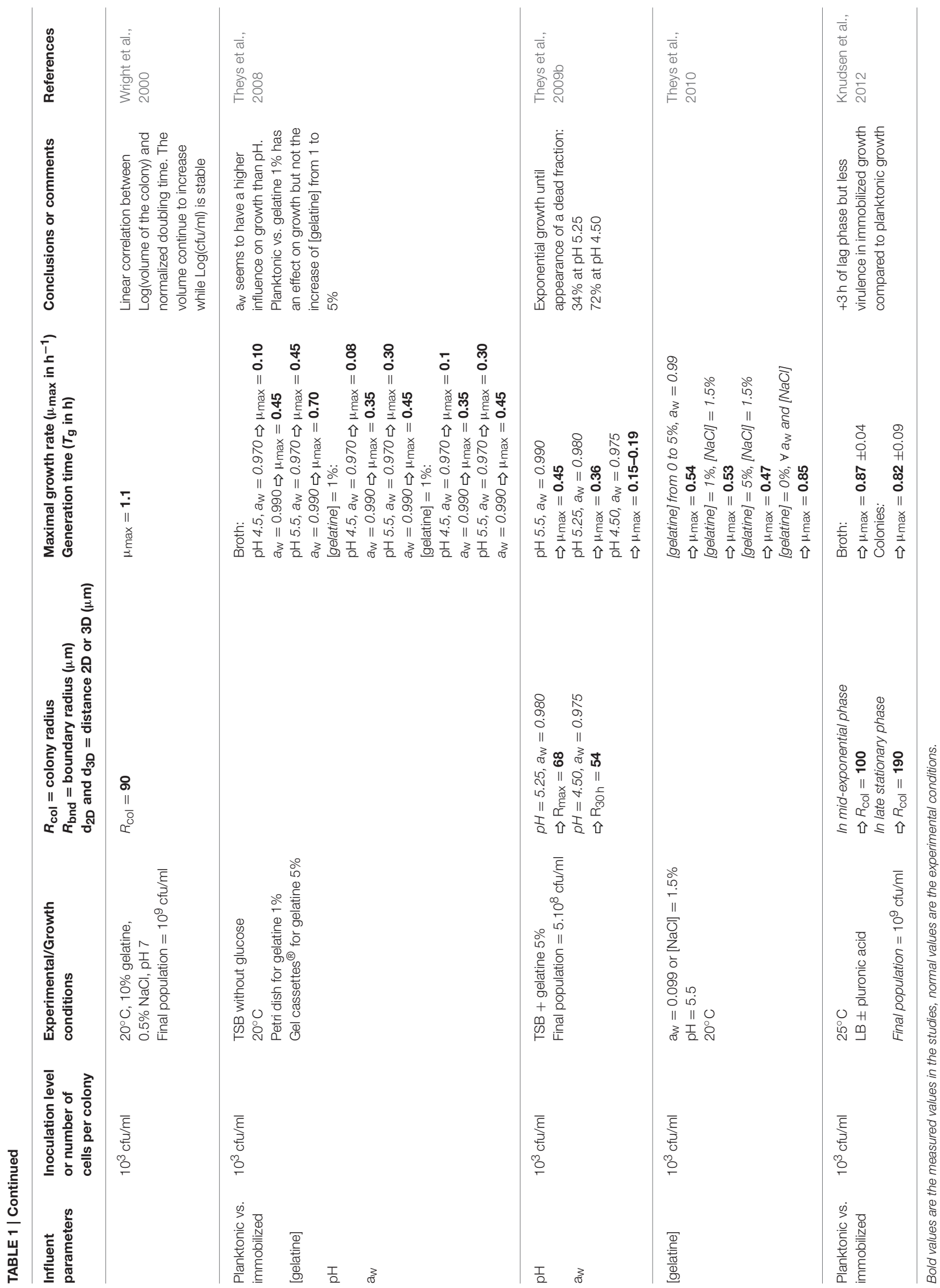


bacteria in gelatine media (Antwi et al., 2007; Mertens et al., 2012; Boons et al., 2013). Van Impe studied mostly large pathogen bacterial colonies grown in agar or gelatine media and used micro-electrodes to measure $\mathrm{pH}$. More recently, high resolution imaging techniques have allowed the (i) exploration of small colonies $(<100 \mu \mathrm{m})$, (ii) measurement of $\mathrm{pH}$ down to a resolution of a few $\mu \mathrm{m}$, and (iii) increasing numbers of monitored parameters like variability of shape, of growth in which single-cell variability, and of metabolism (Bae et al., 2011a; Gonzalez et al., 2012; Knudsen et al., 2012; Koutsoumanis and Lianou, 2013; Ryssel et al., 2013; Vilain et al., 2014). Other research groups have recently compared planktonic and immobilized bacterial growth using molecular techniques to study the difference of gene expression (Knudsen et al., 2012) and protein expression (Knudsen et al., 2012; Vilain et al., 2014). Microcalorimetry has been recently used to study the carbon metabolism at different inoculation levels (Kabanova et al., 2012). The techniques used to study the immobilized bacterial colonies are described in a recent review (Lobete et al., 2015). Imaging fluorescent techniques have allowed the observation of colonies within an opaque matrix such as model cheese. Our group, at the French National Institute for Agricultural Research (INRA, Rennes, France), explores small colonies of lactic acid bacteria (LAB) and their dynamic micro-environment in a model cheese ( $\mathrm{pH}$, diffusion of substrates in and around colonies, etc.) in order to better understand the role of LAB during cheesemaking and ripening at the microscopic scale (Jeanson et al., 2011, 2013; Floury et al., 2013, 2015). We also investigated the role of the size of colonies during ripening by combining omics techniques (Le Boucher et al., 2013, 2015a).

\section{WHAT DOES IMMOBILIZATION IMPLY FOR THE GROWTH OF BACTERIA?}

The growth of colonies has been studied using a qualitative approach and several publications have described how a bacterial colony grew on and within a solid matrix, how they were distributed depending on the inoculation level, and how neighboring colonies interacted with each other either from the same or different species.

\section{Growth of Immobilized Colonies}

Since the first studies, it has been demonstrated that the growth of bacterial colonies on the surface is a concentric pattern (Wimpenny, 1992). Cell division starts from the initial immobilized cell, with the colony expanding progressively at the periphery thus following a concentric pattern (Wimpenny, 1992; Pipe and Grimson, 2008). In the exponential growth phase, the number of cultivable cells is linearly correlated to the Log(colony volume) (Wright et al., 2000; Theys et al., 2009b) for submerged colonies or to the $\log$ (colony area) (Guillier et al., 2006; Skandamis et al., 2007; Mertens et al., 2012) for surface colonies. Image analysis techniques have thus been proposed to replace the time-consuming plating techniques. The height of a bacterial colony growing on a surface of a medium was modeled as a function of the glucose concentration of the medium. Indeed, the glucose concentration is low on the top of the colony. It has been suggested that the growth of bacteria and the development of $\mathrm{pH}$ profiles in and around the colony were determined by the local presence, and diffusion of glucose, in the medium beneath the colony (Wimpenny, 1992). This was the main reason offered to explain why the growth of immobilized cells may be different from that of planktonic cells. It has been demonstrated that most of the mathematical models based on a laboratory broth overestimate the bacterial growth in milk, and even more so its growth in cheese-like media (Theys et al., 2009a).

In conclusion, all the studies on bacterial colony growth have suggested that the growth of colonies (growth rate, final size, and shape) was determined by local concentration of substrates and thus by possible limitations of the diffusion of substrates or end-products in solids (McKay et al., 1997; Walker et al., 1997; Malakar et al., 2002b; Pipe and Grimson, 2008).

\section{Distribution of Colonies: Size of Colonies and Distances between Colonies}

When considering the dimensions of a colony, there are two radii of particular importance: the colony radius from the center of the colony to its periphery $\left(R_{\mathrm{col}}\right)$, and the boundary radius from the center of the colony to the limit of its influence on the medium $\left(R_{\text {bnd }}\right)$ (Malakar et al., 2002a). Figure 1 illustrates these two radii: the colony itself is defined by the radius $\left(R_{\mathrm{col}}\right)$ and its "living space" is defined as the region around the colony $\left(R_{\mathrm{bnd}}\right)$ within which the activity of the bacterial cells is measurable (dashed line), for example by the consumption of substrates and/or production of end-products. The larger the colony (large $R_{\text {col }}$ ), the higher the activity of the colony, the greater the "living spaces" (large $\left.R_{\mathrm{bnd}}\right)$. Furthermore, the larger the radius $R_{\mathrm{bnd}}$, the greater the distance for the substrate to diffuse to reach the colony. The value of $R_{\mathrm{bnd}}$ at the moment of an inoculation of $1 \mathrm{cfu} / \mathrm{ml}$ was estimated to be five times longer that for an inoculation of 100 $\mathrm{cfu} / \mathrm{ml}$ (Malakar et al., 2002a).

The spatial distribution of bacterial colonies is defined by the size of colony and the distances between neighboring colonies. It was measured for the first time in a model cheese, varying with the inoculation levels of a prt ${ }^{-}$strain of Lactococcus lactis ranging from $10^{5}$ to $10^{7} \mathrm{cfu} / \mathrm{ml}$, i.e., within the range used in cheese manufacture (Jeanson et al., 2011). The theoretical distances

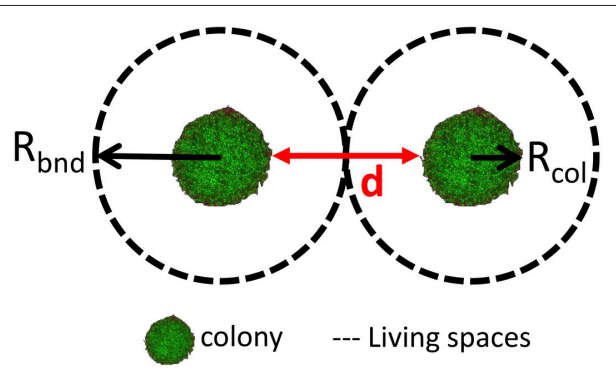

FIGURE 1 | Representation of the colony and its surrounding "living space" (the area within which the colony is active) with the two respective radii $\boldsymbol{R}_{\mathrm{col}}$ and $\boldsymbol{R}_{\mathrm{bnd}} ; \boldsymbol{d}$ is the distance between two neighboring colonies. Adapted from Malakar et al. (2002a) and Wimpenny (1992). 
between colonies were first estimated assuming that (i) all the cells in the inoculum gave rise to a colony and (ii) that they were randomly distributed (Poisson $1 a_{\mathrm{w}}$ ). These two assumptions were confirmed from experimental data obtained by confocal image analysis. It was also demonstrated that the final population was always the same regardless of the inoculation level (Jeanson et al., 2011). As a consequence, the size of colonies was negatively correlated to the level of inoculation, that is, the lower was the inoculation level, the larger the colonies. The distances between neighboring colonies were 123 and $34 \mu \mathrm{m}$ for inoculation levels of $2 \times 10^{5}$ and $9.6 \times 10^{6} \mathrm{cfu} / \mathrm{ml}$, respectively (Table 1 ). A separate study (Kabanova et al., 2012), carried out using agar, gave the spatial distribution parameters from experimental data of a larger scale of inoculation levels of a strain of L. lactis (from $10^{0}$ to $10^{6} \mathrm{cfu} / \mathrm{ml}$ ). The values of distances and radii measured were slightly smaller than those reported by Jeanson et al. (2011) (Table 2). However, the latter used isothermal microcalorimetry which is based on dynamic measurements of heat flow rate. Measurements and calculations of colony radii are mostly in agreement for the strains of the two species (Lactococcus lactis and Streptococcus thermophilus) reaching final populations over $10^{9} \mathrm{cfu} / \mathrm{ml}$ whether they were grown in agar medium or in milk gel/model cheese (Table 2). In the agar medium, the shape of colonies was lenticular; this may explain the difference between the measured and the calculated values. Moreover, the L. lactis strain $\left(\mathrm{lac}^{-} / \mathrm{prt}^{-}\right)$producing Green Fluorescent Protein (GFP) used by Jeanson et al. (2011) produced smaller colonies in the model cheese because it reached a lower final population $\left(5 \times 10^{8}\right.$ $\mathrm{cfu} / \mathrm{ml})$.

For a given inoculation level, the variation of the radii of bacterial colonies followed a Normal distribution centered on the mean radius. Indeed, considering that a colony arises from a single cell, the asynchrony of division of any bacterial culture (Kreft et al., 1998) may explain the variability of the colony radii. Some immobilized cells start their division later than others but all cells stopped to grow at the same time. As a result, different numbers of divisions may occur in neighboring colonies (Koutsoumanis and Lianou, 2013).

\section{Distances between Colonies and Interactions between Different Bacterial Species}

If the distance between two neighboring colonies (denoted as $d$ ) is greater than $R_{\text {bnd }}$, one can consider that there is no interaction between the colonies, but if it is closer one can consider that some level of interaction exists (Figure 2 and Table 1). This applies whether the neighboring colonies comprise the same strain or are formed from different strains or species. Interactions between different species may be in the form of competition for the same substrate (Thomas and Wimpenny, 1996b) or of inhibition because of production of metabolites such as a bacteriocin like nisin (Thomas and Wimpenny, 1996a) or lactic acid (Antwi et al., 2007). This review focuses on the few studies on colonies taking into account the distances between the inhibiting and the affected colonies. Wimpenny et al. (1995) introduced the concept of "propinquity" defined as the maximum distance between neighboring colonies at which there is still interaction.

A strain of Salmonella enterica subsp. enterica serotype Enteritidis (named $S$. Enteritidis thereafter) inhibited a strain of Pseudomonas fluorescens, while a strain L. lactis subsp. lactis inhibited a strain of Listeria monocytogenes on agar media (Wimpenny et al., 1995). The results showed that the inhibition only occurred if the inoculation level of the inhibiting strain was

TABLE 2 | Size of colonies (calculated by microcalorimetric method or measured from micrographs) as a function of different inoculation levels of two different species of lactic acid bacteria grown in agar, milk gels, or in model cheese.

\begin{tabular}{|c|c|c|c|c|c|c|c|}
\hline \multirow{2}{*}{$\begin{array}{l}\text { Inoculation } \\
\text { levels } \\
\text { (cfu/ml) }\end{array}$} & \multicolumn{3}{|c|}{ Agara } & \multicolumn{2}{|c|}{ Model cheese } & \multicolumn{2}{|c|}{ Milk gel $^{d}$} \\
\hline & $\begin{array}{l}R_{\text {col }}(\mu \mathrm{m}) \\
\text { measured }\end{array}$ & $\begin{array}{l}R_{\text {col }}(\mu \mathrm{m}) \\
\text { calculated }\end{array}$ & $\begin{array}{c}\text { Total number } \\
\text { of cells/colony } \\
\text { (calculated) }\end{array}$ & $\begin{array}{c}R_{\text {col }}(\mu \mathrm{m}) \\
\text { measured }^{b}\end{array}$ & $\begin{array}{c}R_{\mathrm{col}}(\mu \mathrm{m}) \\
\text { measured }^{\mathrm{c}}\end{array}$ & $\begin{array}{l}R_{\text {col }}(\mu \mathrm{m}) \\
\text { calculated }\end{array}$ & $\begin{array}{c}\text { Total number } \\
\text { of cells/colon } \\
\text { (calculated) }\end{array}$ \\
\hline $10^{0}$ & 546 & & $1.4 \times 10^{8}$ & & & & \\
\hline $10^{1}$ & $523 \pm 98$ & 150 & $1.2 \times 10^{7}$ & & & $331 \pm 1$ & $1.4 \times 10^{8}$ \\
\hline $10^{2}$ & $192 \pm 16$ & 66 & $2.2 \times 10^{6}$ & & & $160 \pm 4$ & $1.6 \times 10^{7}$ \\
\hline $10^{3}$ & $92 \pm 18$ & 32 & $2.5 \times 10^{5}$ & & $55 \pm 1$ & $74 \pm 0.2$ & $1.6 \times 10^{6}$ \\
\hline $10^{4}$ & $52 \pm 13$ & 14 & $2.3 \times 10^{4}$ & & $32 \pm 4$ & $34 \pm 0.5$ & $1.6 \times 10^{5}$ \\
\hline $10^{5}$ & $25 \pm 3$ & 6 & $1.8 \times 10^{3}$ & $5 \pm 1$ & $16-23$ & $16 \pm 0.3$ & $1.6 \times 10^{4}$ \\
\hline $10^{6}$ & $10 \pm 1$ & 3 & $1.8 \times 10^{2}$ & $3 \pm 1$ & & $7 \pm 0.01$ & $1.6 \times 10^{3}$ \\
\hline $10^{7}$ & & & & $2 \pm 0.2$ & $4 \pm 0.4$ & & \\
\hline
\end{tabular}

a Kabanova et al. (2012): Lactococcus lactis subsp. lactis strain, in CRM agar $35^{\circ} \mathrm{C}, R_{\text {col }}$ and total numbers of cells/colony calculated from the microcalorimetric study for a final population between $10^{9}$ and $10^{10} \mathrm{cfu} / \mathrm{ml}$, $R_{\text {col }}$ measured from micrographs.

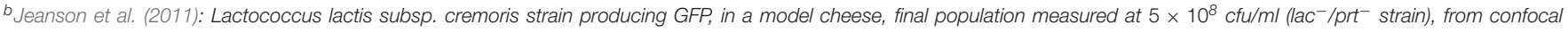
microscopy images.

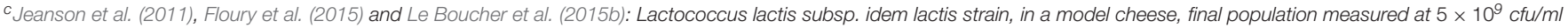
(lac ${ }^{+} / \mathrm{prt}^{+}$strain), from confocal microscopy images.

dStulova et al. (2015): Streptococcus thermophilus strain, in renneted milk gel, calculated final population of between 1.4 and $1.6 \times 10^{9}$ cfu/ml from a microcalorimetric study.

The corresponding total number of cells per colony is also given when calculated in the study. 
above $30-100 \mathrm{cfu} / \mathrm{ml}$ which corresponded to an average distance between the colonies of $1.4-2.2 \mathrm{~mm}$. These results were then confirmed in another study (Thomas et al., 1997) with strains of L. monocytogenes and L. lactis. The Listeria strain was inhibited either by nisin from a nisin-producer Lactococcus strain or, to a lesser extent, by lactic acid production from a non nisin-producer strain. In both cases, the inhibition increased when the distance between colonies of the two species fell from $11 \mathrm{~mm}$ to $100 \mu \mathrm{m}$. A maximum inhibition distance of $5000 \mu \mathrm{m}$ was determined, for the inoculation levels of 12 and 4 cells $/ \mathrm{ml}$ for L. lactis and $L$. monocytogenes, respectively, beyond which there was no further inhibition (Thomas et al., 1997).

In conclusion, as low inoculation levels correspond to the formation of colonies far apart $(d>1.5-5 \mathrm{~mm})$, it has been suggested that for inoculation levels of $100 \mathrm{cfu} / \mathrm{ml}$ and below, no interactions between colonies will occur (Malakar et al., 2000). On the other hand, for an inoculation level greater than 100 $\mathrm{cfu} / \mathrm{ml}$, interactions between colonies can be expected (Figure 2).

\section{GROWTH IN COLONIES: WHEN AND HOW IT DIFFERS FROM PLANKTONIC GROWTH}

The growth of bacteria as colonies is subjected to several constraints that are absent in planktonic cultures, such as a necessary diffusion of substrates through the solid matrix, with potentially limited access to the substrates. Predictive growth models for bacteria have mainly been based around parameters taken from planktonic cultures and led to the observation that they were not applicable for modeling immobilized growth (Pipe and Grimson, 2008; Skandamis and Jeanson, 2015). Attention was thus given to understand when and how immobilized growth differed from planktonic growth, especially under the stressful conditions of the food environment. In this section, two aspects of the consequences of immobilization of bacteria are presented: (i) their responses to conditions of stress and (ii) on the microheterogeneity of the micro-environment inside and around the colonies.

\section{Narrower Boundaries of Growth/No Growth Regions under Stressful Conditions}

The environment existing in food products rarely provides optimal conditions for the growth of microorganisms. The main factors affecting the bacterial growth in food are temperature, $\mathrm{pH}, \mathrm{NaCl}$ concentration, water activity $\left(a_{\mathrm{w}}\right)$ and substrate concentration. Increasing the $\mathrm{NaCl}$ or sucrose concentrations also decreases the $a_{\mathrm{w}}$ and increases the osmotic pressure, with combined negative effects. Several studies have modified these parameters to determine the conditions leading to growth and no growth conditions comparing planktonic and immobilized bacterial growth. Most of these studies have focused on pathogenic species, aiming at predicting or preventing their growth in food. The experimental details and results from the most cited studies in the literature are listed in Table $\mathbf{1 .}$

The growth of a strain of Salmonella enterica subsp. enterica serotype Typhimurium (named $S$. Typhimurium thereafter) in gelatine medium was compared to its growth in broth, at different

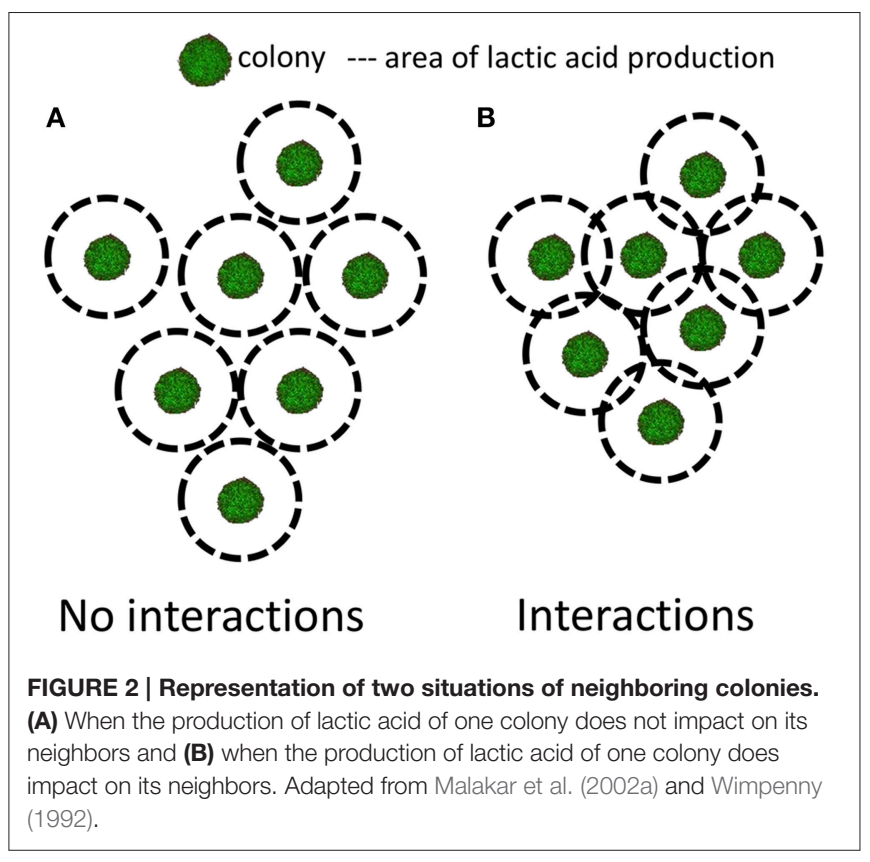

conditions of $\mathrm{pH}$ and $\mathrm{NaCl}$ (Brocklehurst et al., 1995). The results show that $S$. Typhimurium behaves the same when growing in colonies and in a planktonic culture when under optimal conditions ( $\mathrm{pH}=7$ and $\mathrm{NaCl}$ concentration of $0.5 \%$ ). However, the generation time $t(t=\log 2 / \mu$ where $\mu$ is the growth rate) was increased by a factor between 1.3 and 2 in the more stressful conditions ( $\mathrm{pH}=5$ and $\mathrm{NaCl}$ concentration of $3.5 \%$ ). The growth rates of this bacterial strain were thus ordered as follow: $\mu_{\text {planktonic }}>\mu_{\text {submerged }}>\mu_{\text {surface }}$ regardless of the $a_{\mathrm{w}}$ when the $\mathrm{NaCl}$ concentration was $0.5 \%$, and regardless of the $\mathrm{NaCl}$ concentration for maximum $a_{\mathrm{w}}$ (Brocklehurst et al., 1997). The maximum viable cell counts were less affected by a low value of $a_{\mathrm{w}}$ reduced by high sucrose and $\mathrm{NaCl}$ concentrations if the colony was submerged rather than on the surface. An explanation could be that the substrates are only accessible through the small area of the underside of surface colonies, whilst it is accessible all around the colony on a bigger area when submerged. By comparing a strain of $S$. Typhimurium growing as submerged colonies or in planktonic culture, it was shown that the $a_{\mathrm{w}}$ was the most influential parameter on the growth rates (Theys et al., 2008). However, decreasing $a_{\mathrm{w}}$ by increasing $\mathrm{NaCl}$ concentration was relatively more harmful to the growth of colonies, because of the combined effect on osmotic pressure, than by increasing gelatine concentrations of the media (Theys et al., 2010). In agreement with the latter, a lower growth rate of growth was observed in planktonic cultures than in submerged colonies of a strain of $S$. Typhimurium and the growth in colonies increased its sensitivity to the inhibition exerted by oregano oil (Skandamis et al., 2000). Surprisingly, the growth rate of submerged colonies of $S$. Typhimurium was found lower in broth than in agar medium, but lower in gelatine medium than in broth (Walker et al., 1998). Furthermore, when the growth rate was not affected by the immobilization of bacteria, the lag phase was increased in comparison to planktonic growth (Knudsen et al., 2012; Nielsen 
et al., 2013). Figure 3 is an example of the detrimental effect of immobilization of bacteria on their growth when under severe conditions such as low $\mathrm{pH}$ and high concentration of $\mathrm{NaCl}$.

Similarly, a strain of $L$. monocytogenes always displayed a lower growth rate when in submerged colonies than in the planktonic form regardless of the sucrose concentration (ranging from 0 to $60 \%$ ) and the initial $\mathrm{pH}$ of the medium. Furthermore, the minimal $\mathrm{pH}$ for enabling growth was higher $(\mathrm{pH}=5)$ in colonies than in a planktonic culture (Meldrum et al., 2003). L. monocytogenes growth was also shown to be affected by immobilization at low $\mathrm{pH}$ and low $a_{\mathrm{w}}$ (Koutsoumanis et al., 2004) as shown on Figure 4. However, in this study, $a_{\mathrm{w}}$ had been decreased by increasing the $\mathrm{NaCl}$ concentration, the harmful effects of both the $\mathrm{NaCl}$ and a low $a_{\mathrm{w}}$ were thus combined.

The growth of a strain of Listeria innocua inoculated at $10^{3}$ $\mathrm{cfu} / \mathrm{ml}$ in milk and in gelatinized milk was compared. The growth rates substantially decreased when the concentration of gelatine in the medium was raised from 0 to 50\% (Theys et al., 2009a). Under the same conditions, a strain of L. lactis was even more detrimentally affected by the increase in gelatine in pasteurized

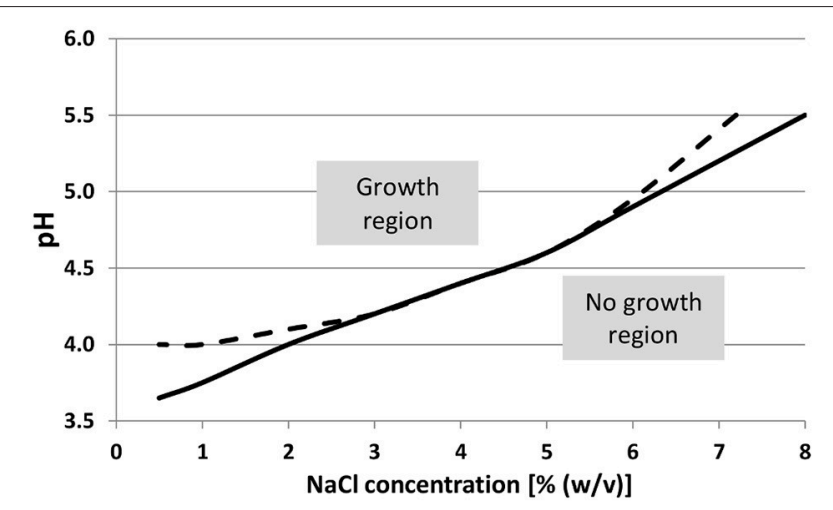

FIGURE 3 | Growth/no growth regions of Salmonella Typhimurium in TSB (tryptic soy broth) at $20^{\circ} \mathrm{C}$ as a function of $\mathrm{pH}$ and $\mathrm{NaCl}$ concentrations, with gelatine concentrations of 0 and $50 \mathrm{~g} / \mathrm{l}$. Adapted from Theys et al. (2010).

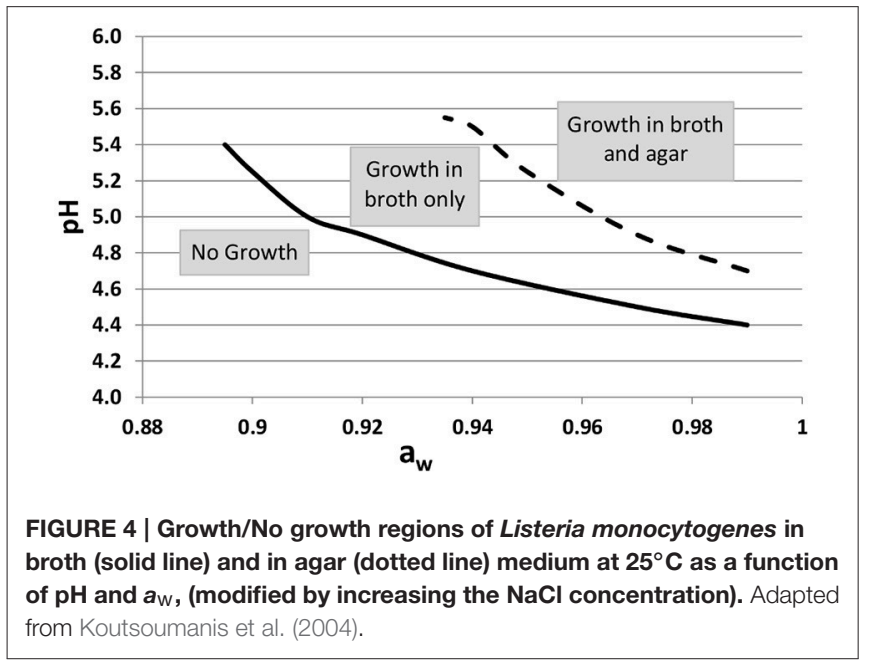

milk (Antwi et al., 2007). The same conclusions were drawn for two LAB strains: the growth rate as colonies was lower than in a broth but only when the inoculation level was lower than $10^{3} \mathrm{cfu} / \mathrm{ml}$ for L. lactis (Kabanova et al., 2012) or lower than $100 \mathrm{cfu} / \mathrm{ml}$ for Lactobacillus curvatus (Malakar et al., 2002a). Two studies, using the same Lactococcus strain grown in M17 media, showed that, for inoculation levels above $10^{2}$ or $10^{4} \mathrm{cfu} / \mathrm{ml}$ (Kabanova et al., 2012, 2013): (i) there was no glucose diffusion limitation in agar at $1 \%$, (ii) the value of $\mu_{\max }$ in agar was similar to that in broth during the exponential growth phase, (iii) the LAB strain switched to a heterofermentative metabolism in agar, thus producing less lactic acid with the same amount of glucose, and stopping growth at a higher $\mathrm{pH}$ in agar (Kabanova et al., 2013).

The conclusion from all these results is that the growth of bacteria in colonies differs from the planktonic growth, (i) below a specific inoculation level (depending on the species or the strain of bacteria) and (ii) especially in stressful conditions because of narrower boundaries of conditions conducive to growth.

\section{Heterogeneity in and Around Colonies (Growth, pH, Oxygen)}

The heterogeneity in and around the colonies results from different aspects of the bacterial activity: growth rates (or lysis), substrate consumption and metabolic activity. The potential existence of microgradients within and around the colony would suggest that the environmental conditions $(\mathrm{pH}$, oxygen, redox potential, etc.) experienced by the cells of the colony are not those of the mean values for the medium (Hills, 2001). The metabolic action, either with respect to the consumption of substrates or the production of end-products, is likely to create microgradients of concentration that cause the heterogeneity of bacterial activity inside the colony. Firstly, the studies about the heterogeneity of growth and metabolic activity inside colonies are discussed. Then, with the technical evolution from micro-electrodes to the recent imaging techniques, the possible existence of microgradients in the environmental parameters inside and around the colony is discussed. These parameters include the $\mathrm{pH}$, resulting from production of lactic acid, and oxygen concentration, resulting from its consumption by bacteria. In order to measure the different types of spatial heterogeneity, all studies were performed on large colonies, mostly on the surface of agar/gelatine media (see Table 1 for details).

\section{Heterogeneity of Growth Rate and Metabolic Activity between Cells of the Colony}

Two types of heterogeneity within the colony have been shown: (i) a gradient of growth rates or metabolite production from the center to the periphery of the colony arising because of the concentrical growth pattern (Wimpenny, 1992), and (ii) a random heterogeneity due to random differences of division or gene expression between cells (Mikkelsen et al., 2007) which has been observed even in small colonies. Different aspects of the heterogeneity can be observed: morphology, growth rates, or metabolic activity (metabolite pattern). 
For large colonies, rings exhibiting different morphologies were described $\left(R_{\mathrm{col}}=750 \mu \mathrm{m}\right)$ for Escherichia coli with cells modifying their morphology when aging (Shapiro, 1987), as well as rings with different cell densities for colonies $\left(R_{\mathrm{col}}=250\right.$ $450 \mu \mathrm{m}$ ) of different species of Bacillus (Kim et al., 2014). The spatial heterogeneity of colony growth, between active growth for the periphery cells and maintenance activity for the central cells where glucose was scarce, was modeled for Bacillus (Kreft et al., 1998). Growth rates were measured in the center and at the periphery of a large $\left(R_{\mathrm{col}}>200 \mu \mathrm{m}\right)$ colony of $S$. Typhimurium (McKay et al., 1997). Soon after the formation of the colony $(13 \mathrm{~h})$, the growth rate at its periphery was twice that of the center, demonstrating that the periphery of a large colony was the region of maximum metabolic activity (Figure 5 and Table $\mathbf{1}$ ). The growth slowed down in the center of the colony due to the accumulation of lactic acid possibly combined with the depletion of glucose or carbon sources.

Metabolic heterogeneity has been described by the observation of gradients in lysis activity, as well as gradients of metabolite production or enzyme activity within the colony. An intense lysis of cells was observed in the center of colonies of Vibrio cholerae by using a vital stain of the cells (Wimpenny, 1992). Large surface colonies $\left(R_{\text {col }} \approx 350 \mu \mathrm{m}\right)$ of Enterobacter cloacae were sliced $(10 \mu \mathrm{m})$ from top to bottom, to measure the $\mathrm{NADH}$ oxidase activity (Wimpenny, 1992). As oxygen is more available at the surface of the colony, higher activities were found in the upper $100 \mu \mathrm{m}$ layer. The same conclusion was drawn from using Fourier transform infrared (FT-IR) microspectroscopic mapping of large colonies of Bacillus megaterium (obligate aerobes) and Legionella bozemanii (microaerophiles). The cells at the top and in the center bottom layers (the "oldest" cells) got the maximum concentrations of capsule components for $B$. megaterium and of poly- $\beta$-hydroxybutyric acid, a storage material present in intracellular granules, for L. bozemanii (Ngo Thi and Naumann, 2007). In E. coli colonies $\left(R_{\mathrm{col}}=1 \mathrm{~mm}\right)$, vibrational spectroscopy spectra also showed that for the oldest cells in the surface layers, the RNA level was lower than that in younger cells in the deeper layers (Choo-Smith et al., 2001).

For smaller colonies, results are less clear. For example, the adenylate pool which includes ATP has been shown to be affected by the growth in submerged colonies of $S$. Typhimurium (Walker

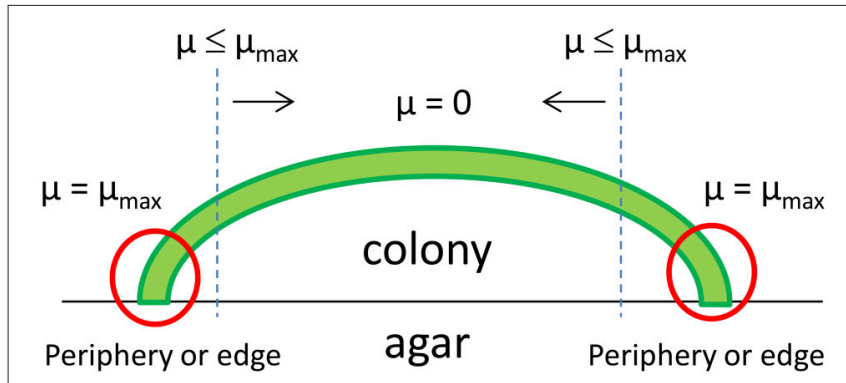

FIGURE 5 | Simplified model illustrating the spatial variations in the specific growth rate $(\mu)$ within a growing bacterial colony of a facultative anaerobe, such as Salmonella Typhimurium. Adapted from Mckay et al. (1997). et al., 1998). The authors suggested that the variation of adenylate production through incubation time, in comparison with broth culture, could be due to an heterogeneity within colonies but this heterogeneity has never been proved. In small colonies $\left(R_{\mathrm{col}}=\right.$ $40-60 \mu \mathrm{m})$ of $E$. coli, three distinct zones could be observed (center, intermediate and edge) using FT-IR spectra (Ngo-Thi et al., 2003). On the other hand, elastic-scattering patterns of small colonies $\left(R_{\mathrm{col}}=50-100 \mu \mathrm{m}\right)$ of L. monocytogenes, $E$. coli, and Salmonella Montevideo, showed no microgradients of metabolite concentration (Ngo-Thi et al., 2003; Bae et al., 2011a), indicating that their mean profile was representative of the whole colony. Small colonies $\left(R_{\mathrm{col}}=25 \mu \mathrm{m}\right)$ of $E$. coli have also been shown to be quite homogeneous using vibrational spectroscopy and it was even suggested that $6 \mathrm{~h}$ colonies were the most suitable for building an identification data base (Choo-Smith et al., 2001). Identification at early stage of growth of bacterial colonies was possible using a new highly sensitive and non-destructive technique, chromatic confocal microscopy (Drazek et al., 2015).

Finally, the variability of phenotype randomly occurs when a sub-population develops under stressful conditions, either in colonies or in planktonic cultures. This phenomenon was observed under acid stress conditions for small colonies of $L$. plantarum (Ingham et al., 2008) and for B. cereus under severe salt stress (den Besten et al., 2007). Heterogeneity of division and shape was observed in small colonies of L. brevis (from an initial cluster of a few cells through to several generations) after exposure to an oxidizing disinfectant (Zhao et al., 2014). In small colonies of six different strains of $L$. lactis, the area of dead cells, measured using propidium iodide in microscopy, correlated with growth rates. The dead cells were randomly distributed until $38 \mathrm{~h}$, and were then concentrated in the center of the colony at $134 \mathrm{~h}$ (Ryssel et al., 2013).

In conclusion, putting aside the natural random variability of phenotype, these results show, by mapping the growth and the metabolites of large colonies $\left(R_{\text {col }}>250 \mu \mathrm{m}\right)$, that cells differentiate during the stage of growth within the colony. For this reason, small colonies are homogeneous because all cells exhibit the same growth state.

\section{Gradients of $\mathrm{pH}$ in and Around Colonies}

The production of lactic acid from bacteria has often been suggested to be the reason why growth stops, due to the accumulation of lactic acid in and around colonies. Using micro-electrodes and then $\mathrm{pH}$-sensitive fluorophores, $\mathrm{pH}$ microgradients were recorded only in the case of large colonies, in and around colonies grown on agar/gelatine. However, the question remained if there were also $\mathrm{pH}$ microgradients around small colonies or in food such as cheese.

Using micro-electrodes, the first $\mathrm{pH}$ profiles were performed only on large colonies because of the poor resolution of the technique. Microgradients of $\mathrm{pH}$ were observed in and around large colonies $\left(R_{\mathrm{col}} \approx 10 \mathrm{~mm}\right)$ of Bacillus cereus (Wimpenny, $1992)$ and large surface colonies $\left(R_{\mathrm{col}}=800 \mu \mathrm{m}\right)$ of $S$. Typhimurium (Walker et al., 1997). Inoculated at $1 \mathrm{cfu} / \mathrm{ml}$ with supplementation of glucose at $1 \%$, a difference of $2 \mathrm{pH}$ units was generated between the center of the colony and the edge of the gel (a distance of $1.2 \mathrm{~mm}$ ); this difference was only $0.5 \mathrm{pH}$ 
units in a medium supplemented with glucose at $0.1 \%$ (Figure 6 and Table 1). For submerged colonies $\left(R_{\mathrm{col}}=200 \mu \mathrm{m}\right)$ of $S$. Typhimurium in agar gels, Wimpenny et al. (1995) observed a span of $\mathrm{pH}$ of $0.8 \mathrm{pH}$ units from the periphery of the colony to the surface of the agar gel (a distance of $3 \mathrm{~mm}$ ). In contrast, colonies of $S$. Typhimurium inoculated at $10^{3} \mathrm{cfu} / \mathrm{ml}\left(R_{\mathrm{col}}=200 \mu \mathrm{m}\right) \mathrm{did}$ not generate measurable $\mathrm{pH}$ gradients but modified the $\mathrm{pH}$ in the whole bulk medium (Walker et al., 1997). Using ratio-imaging fluorescence, $\mathrm{pH}$ microgradients were observed in and around submerged colonies of $L$. curvatus when inoculated at between 5 and $100 \mathrm{cfu} / \mathrm{ml}$ (leading to colonies of $R_{\mathrm{col}}=215$ and $190 \mu \mathrm{m}$, respectively) but no $\mathrm{pH}$ variation was observed when inoculated at $1000 \mathrm{cfu} / \mathrm{ml}\left(R_{\mathrm{col}}=75 \mu \mathrm{m}\right)$ (Malakar et al., 2000).

In order to confront the observations in agar and gelatine to a real food medium, $\mathrm{pH}$ was measured at the microscopic level in a model cheese and in real commercial cheeses. Using ratio-imaging fluorescence, local $\mathrm{pH}$ was measured during the acidification of colonies of $L$. lactis whose radii ranged from 17.5 to $55.5 \mu \mathrm{m}$, corresponding to the lowest inoculation levels possible in cheesemaking, ranging from $1.3 \times 10^{3}$ to $1.6 \times$ $10^{5} \mathrm{cfu} / \mathrm{ml}$, respectively (Jeanson et al., 2013). Regardless of the observed colony size, no $\mathrm{pH}$ microgradients could be observed around colonies (Figure 7). Furthermore, in the same model cheese, the same strain of $L$. lactis displayed no evidence of acid stress at the gene expression level (Cretenet et al., 2011). These results are in agreement with those described above and observed in a gelatine medium for colonies of L. curvatus up to $150 \mu \mathrm{m}$ (Malakar et al., 2000). These consistent results demonstrate that the diffusion of lactic acid was not the limiting factor for growth neither in gelatine nor in a model cheese containing colonies which radius was smaller than $150 \mu \mathrm{m}$. Furthermore, in ripened commercial Cheddar cheeses, $\mathrm{pH}$ microgradients have been observed at the microscopic scale of a few $\mu \mathrm{m}$ using the fluorescence life-time (FLIM), but not especially around colonies

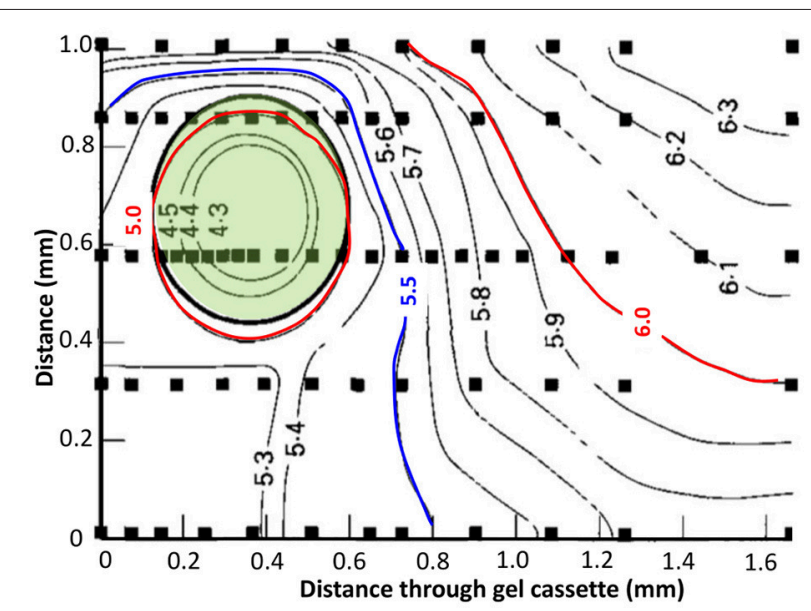

FIGURE 6 | pH profile through a 2-day old colony of Salmonella Typhimurium, inoculum density $1 \mathrm{cell} / \mathrm{ml}$, initial $\mathrm{pH} 7.0$, glucose at $1 \%$ (w/v). Solid squares indicate points where actual measurements were taken. Solid lines indicate $\mathrm{pH}$ isopleths which represent an approximation of where the $\mathrm{pH}$ gradients may lie. The green area shows colony location. Adapted from Walker et al. (1997).
(Burdikova et al., 2015). The accumulation of lactic acid around the colonies has been suggested as the main explanation for the lower growth rate in renneted milk gels when compared with that in liquid milk (Stulova et al., 2015). The simplified composition (no fat, no $\mathrm{NaCl}$ ) and the homogeneous structure of the model cheese (Jeanson et al., 2013) may explain the non-accumulation of lactic acid around small colonies whilst in commercially available cheeses (Burdikova et al., 2015), lactic acid concentration may vary at the microscopic scale because of a more heterogeneous microstructure.

\section{Gradients of Oxygen Concentration around Colonies}

Oxygen $\left(\mathrm{O}_{2}\right)$ is one of the most important parameters for determining the behavior of bacterial growth. Depending on the species, $\mathrm{O}_{2}$ can be favorable to growth (aerobes) or inhibiting (anaerobes), or even "neutral" (microaerophilic). For example, for facultative anaerobes such as $S$. aureus or E. coli, the cell division has been shown to be more intense on the bottom layer of the colony where $\mathrm{O}_{2}$ is scarce and substrates are abundant (Reyrolle and Letellier, 1979). On the other hand, for aerobes such as Pseudomonas putida, the top layer of the colony was the zone of the most intense cell division (Reyrolle and Letellier, 1979). Oxygen gradients were first measured inside a colony of $B$. cereus in 1983 using micro-electrodes (Pipe and Grimson, 2008). It has been measured mainly on large surface colonies because $\mathrm{O}_{2}$ is present over the whole surface of the colony. The $\mathrm{O}_{2}$ concentration decreases with depth moving within the colony and also in depth through the medium below and around the colony in all directions (Wimpenny, 1992).The aerobic zone is considered to exist through $30-40 \mu \mathrm{m}$ depth in a gelatine medium (Walker et al., 1997). However, Tammam et al. (2001), questions the use of micro-electrodes because they can give non-reproducible results due to the poisoning of the platinum electrodes by other ions. Instead, these authors developed in situ mass spectroscopy measurements to investigate the concentrations of $\mathrm{O}_{2}$ and $\mathrm{CO}_{2}$ concentrations in MRS agar inoculated with a strain of $L$. paracasei (Tammam et al., 2001). Their results show that $\mathrm{O}_{2}$

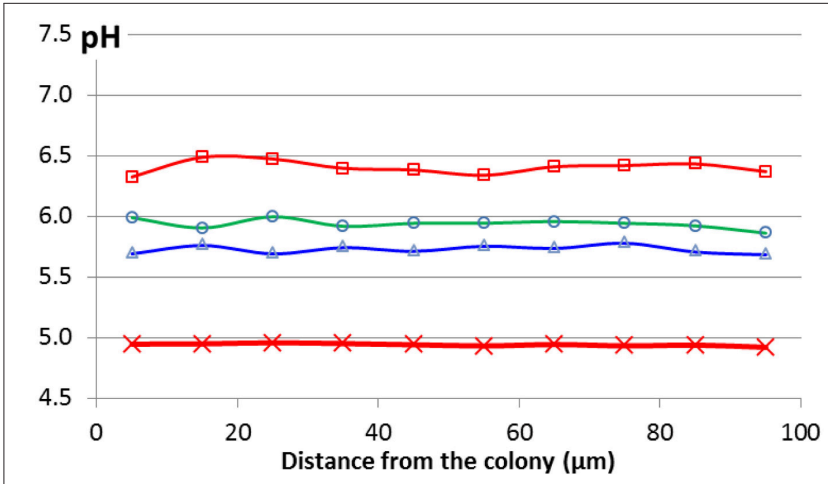

FIGURE 7 | pH profiles measured using a pH-sensitive fluorophore (C-Snarf-4) and confocal microscopy for a colony (radius $=65 \mu \mathrm{m}$ ) growing in a model cheese throughout acidification: $19 \mathrm{~h}(\square), 24 \mathrm{~h}(\bigcirc)$, $26 \mathrm{~h}(\Delta)$, and all measurements from 42 to $72 \mathrm{~h}$ (red bold line, $X$ ). Adapted from Jeanson et al. (2013). 
was rapidly consumed by $\mathrm{LAB}$ metabolism, while $\mathrm{CO}_{2}$ was produced as expected. They showed that in the aerobic zone, there was a gradient of $\mathrm{O}_{2}$ concentration through a $5 \mathrm{~mm}$ depth in agar after $24 \mathrm{~h}$ of inoculation whilst gradients of $\mathrm{CO}_{2}$ concentration occurred in the same zone but through a $20 \mathrm{~mm}$ depth (Figure 8).

For the first time in Cheddar cheeses, these authors also investigated the evolution of the concentrations of $\mathrm{O}_{2}$ and $\mathrm{CO}_{2}$ at depth just below the rind (Tammam et al., 2001). These innovative results concluded that the $\mathrm{O}_{2}$ concentration ranged between 350 and $0 \mu \mathrm{M}$ between the surface of the cheese and $16 \mathrm{~mm}$ depth, respectively, after 2 days of ripening. After 15 days, no $\mathrm{O}_{2}$ could be measured at a depth of $4 \mathrm{~mm}$ (Figure 9). The small colonies of lactococci, observed within the curd by confocal microscopy, were suggested as responsible for the consumption of $\mathrm{O}_{2}$ leading to the decrease of the redox potential known in Cheddar cheese manufacture, for example (Caldeo and McSweeney, 2012). The $\mathrm{CO}_{2}$ concentration was also directly linked to the heterofermentation of lactococci colonies, which produced up to $16 \mathrm{mM}$ of $\mathrm{CO}_{2}$ after 200 days of ripening at a depth of $15 \mathrm{~mm}$. A chemically reducing environment, (i.e., low redox potential), in cheese has been suggested to be essential in the development of flavor and stability (Kristoffersen, 1985). However, in contrast to $\mathrm{pH}$, local variation of the redox potential around colonies has never been investigated at the microscopic scale.

In conclusion, it seems clear that heterogeneity can occur within and around the colonies of bacteria with respect to several parameters directly linked to the bacterial metabolic activity. However, the size of the colonies, and thus the inoculation level, is a major factor determining heterogeneity and the existence of such microgradients.
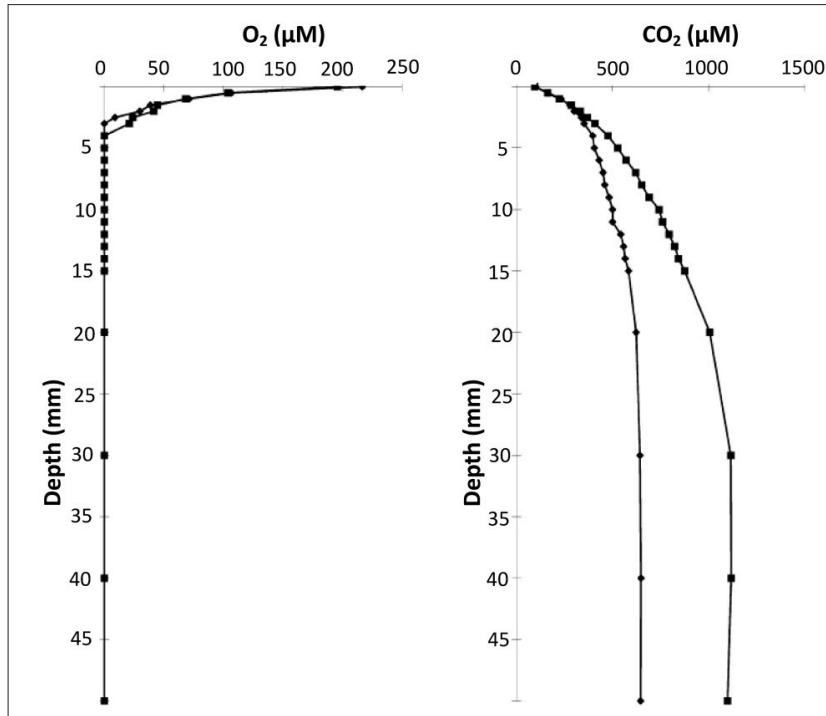

FIGURE $8 \mid \mathrm{CO}_{2}$ and $\mathrm{O}_{2}$ concentration profiles with depth at $24 \mathrm{~h}(\diamond)$ and $48 \mathrm{~h}(\square)$ after inoculation with Lactobacillus paracasei CI3 in MRS $0.1 \%$ agar. A MIMS (membrane inlet mass spectrometric) probe was inserted through column of growth. Adapted from Tammam et al. (2001).

\section{DIFFUSION LIMITATIONS WITHIN THE SOLID MATRICES}

To sustain the growth of bacteria in colonies, substrates have to diffuse from the solid (food) matrix to the colony. At the same time, end-products have to diffuse away from the colony to the matrix, especially if they inhibit bacterial growth such as lactic acid.

The existence of diffusion limitations is the first hypothesis put forth to explain slower growth of the cells in the center of the colony and the microgradients arising in and around the colony. This paradigm has been widely used by different groups to explain their results (Brocklehurst et al., 1997; McKay et al., 1997; Walker et al., 1997; Stecchini et al., 1998; Malakar et al., 2000; Pipe and Grimson, 2008; Kabanova et al., 2012). Even if microgradients of $\mathrm{pH}$ and $\mathrm{O}_{2}$ have been measured, to our knowledge, microgradients of redox potential, inhibitors, or substrates have not, and their existence is still to be shown. Furthermore, some of these studies initially suggested diffusion limitations of the substrates, but then concluded, in the case of numerous and small colonies in favorable growth conditions, that there were no mass transfer limitations of substrates and lactic acid (Stecchini et al., 1998; Malakar et al., 2002b; Kabanova et al., 2012, 2013). For instance, Malakar et al. (2002b) concluded after they measured the effective diffusion coefficient of lactic acid in gelatine medium that the diffusion of lactic acid was not limiting for growth, and that the growth rate was determined only by the generation time of $L$. curvatus, a LAB strain. They obtained a mean diffusion coefficient of $2.81 \times 10^{-10} \mathrm{~m}^{2} / \mathrm{s}$ in MRS with $10 \%$ gelatine at $20^{\circ} \mathrm{C}$, which they compared with

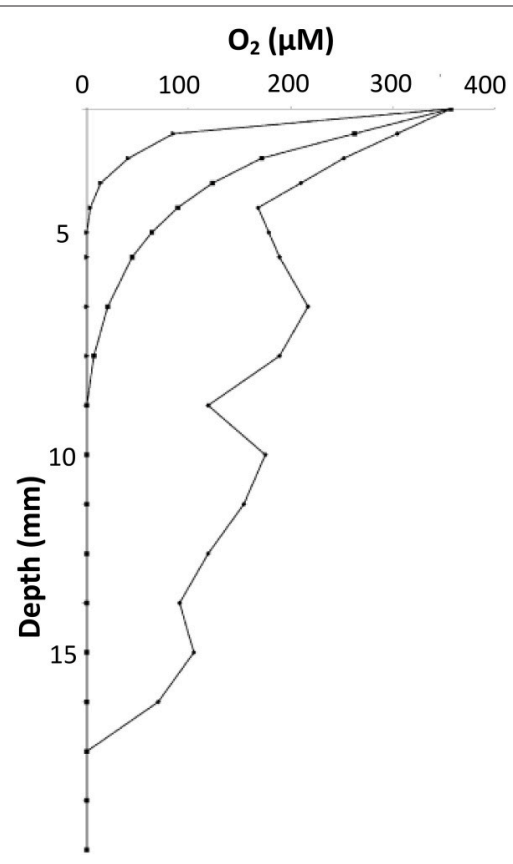

FIGURE $9 \mid \mathrm{O}_{2}$ concentration profiles under the rind of Cheddar cheese at 2 days $(\$), 9$ days $(\square)$, and 15 days $(\Delta)$ of maturation. Adapted from Tammam et al. (2001). 
a diffusion coefficient of $1.74 \times 10^{-10} \mathrm{~m}^{2} / \mathrm{s}$ in water at $25^{\circ} \mathrm{C}$ previously measured by Cussler (1997). Therefore, Malakar et al. (2002b) concluded that because both values were of the same order of magnitude in the gel and in the aqueous solution, lactic acid produced by bacteria can easily diffuse through gels. This conclusion is questionable though, because others studies such as those conducted by Ribeiro et al. (2005) and Øyaas et al. (1995) reported values of diffusion coefficient of lactic acid in water much higher than that of Cussler (1997) of between $7 \times 10^{-10}$ and $10 \times 10^{-10} \mathrm{~m}^{2} / \mathrm{s}$ at $25^{\circ} \mathrm{C}$, meaning that diffusion of lactic acid in the gel media was around 4-6 times lower than in the aqueous solution. It may only be for large colonies (more than $10^{5}$ cells/colony or $100 \mathrm{cfu} / \mathrm{ml}$ ), producing a large amount of lactic acid, that mass transfer limitations can be significant (Malakar et al., 2002a). Furthermore, the diffusion coefficient of glucose at $5^{\circ} \mathrm{C}$ in a $0.8 \%$ agar medium was $3.27 \times 10^{-10} \mathrm{~m}^{2} / \mathrm{s}$ and was found to decrease linearly with an increase of the agar concentration (Mignot and Junter, 1990). These results demonstrated that the diffusion coefficient of glucose was dependent on the gel microstructure because it decreased with the pore size of the gel network. On the other hand, the diffusion rate of glucose and a small protein (insulin-like growth factor) was shown to be independent of the pore size of the gel with an increased concentration of agar (Stecchini et al., 1998). Finally, the little number of studies on diffusion in gels does not allow clear conclusions on the limiting effect of diffusion of substrates or inhibitors.

In cheese, diffusion of small molecules (water, $\mathrm{NaCl}$, lactose) has been studied while knowledge on diffusion of macro-molecules lacks of data (Floury et al., 2010). Recently, Fluorescence Recovery After Photobleaching was adapted to a model cheese (Floury et al., 2012) in order to measure the diffusion coefficients of fluorescent dextrans of different molecular sizes as well as a range of milk proteins. The major conclusion was that the dextrans (which are flexible and charge-neutral molecules) as large as $2000 \mathrm{kDa}$ were able to diffuse through the model cheese as well as the milk proteins (which are rigid and charged molecules). However, the milk proteins were more hindered in the cheese protein network than dextran molecules of similar hydrodynamic radii (Silva et al., 2013). From these studies, it remains very difficult to draw specific conclusions about the potential effects of diffusion limitations of substrates or end-products on bacterial growth and metabolic activity. Indeed, these diffusion rates have now to be compared to enzymatic reaction rates in immobilized conditions, which are, to our knowledge, still unknown and difficult to determine experimentally. We can only suggest that diffusion within the model cheese matrix is probably not the most limiting factor for the growth of cells at the periphery of colonies where the concentration of the substrates is very high. However, one can wonder what happens to the molecules, especially large molecules, upon reaching the center of the colony. In other words, is the colony porous enough to large molecules, either to penetrate the colony or to be expelled from the colony when released after bacterial lysis?

\section{INTEGRATED ANALYSIS AND NEW CONCEPTS OF THE BEHAVIOR OF BACTERIAL COLONIES}

This section outlines the consequences of the immobilization of bacteria in colonies on their growth and metabolic activity in order to identify general principles and theoretical concepts of importance for fermented food products.

\section{How the Spatial Distribution of Colonies Has a Crucial Impact on Growth}

When immobilized as colonies in a solid matrix, bacteria experience multiple constraints on their growth pattern: they develop as colonies and diffusion limitations may limit their access to the substrates. Micro-colonies have previously been defined as colonies displaying a radius $R_{\mathrm{col}}$ as small as $1.5 \mu \mathrm{m}$ up to $100 \mu \mathrm{m}$ (Choo-Smith et al., 2001; Bae et al., 2011b; Zhao et al., 2014) and macro-colonies as those with a radius as large as $2.5 \mathrm{~mm}$ (Ngo Thi and Naumann, 2007). However, all these studies were either focused on micro- or on macrocolonies but never integrated data on both. The present overview of literature led to the conclusion that micro- and macrocolonies were two different conditions of growth depending on a threshold of size, determined by the initial level of population. Figure 10 illustrated the two conditions of colonies along with the planktonic form of culture for comparison, defined as follows:

- Large colonies or macro-colonies $=>$ colony radii that are generally above a threshold of $100-200 \mu \mathrm{m}\left(R_{\mathrm{col}}>100-\right.$ $200 \mu \mathrm{m}$ ), or typically more than $10^{5}$ cells per colony, usually generated by inoculation levels or initial populations below $10^{2}-10^{3} \mathrm{cfu} / \mathrm{ml}$

- Small colonies or micro-colonies $=>$ colony radii that are generally below $100-200 \mu \mathrm{m}\left(R_{\mathrm{col}}<100-200 \mu \mathrm{m}\right)$, or typically less than $10^{4}$ cells per colony, usually generated by inoculation levels or initial populations above $10^{3}-10^{4} \mathrm{cfu} / \mathrm{ml}$.

The threshold between micro-colonies and macro-colonies is determined by the inoculation level above which growth in optimal conditions resembles to planktonic growth. The precise threshold depends on the bacterial species, but implies an inoculation level of between $10^{2}$ and $10^{4} \mathrm{cfu} / \mathrm{ml}$.

The hypothesis of diffusion limitations around colonies seems relevant for macro-colonies but not for micro-colonies as the growth rate of bacteria is then comparable to that in the exponential phase of planktonic growth (McKay and Peters, 1995; Malakar et al., 2002a; Kabanova et al., 2012, 2013). On the one hand, if micro-colonies on agar/gelatine media display the same growth rate as that for planktonic growth, the most likely hypothesis is that the substrates can penetrate inside microcolonies so that all the cells have access to the substrates. On the other hand, if there is heterogeneity of growth rates inside the macro-colonies, the hypothesis is that some of the substrates, most likely the larger molecules, do not reach the center of the colony so that those cells cannot access such substrates. These 


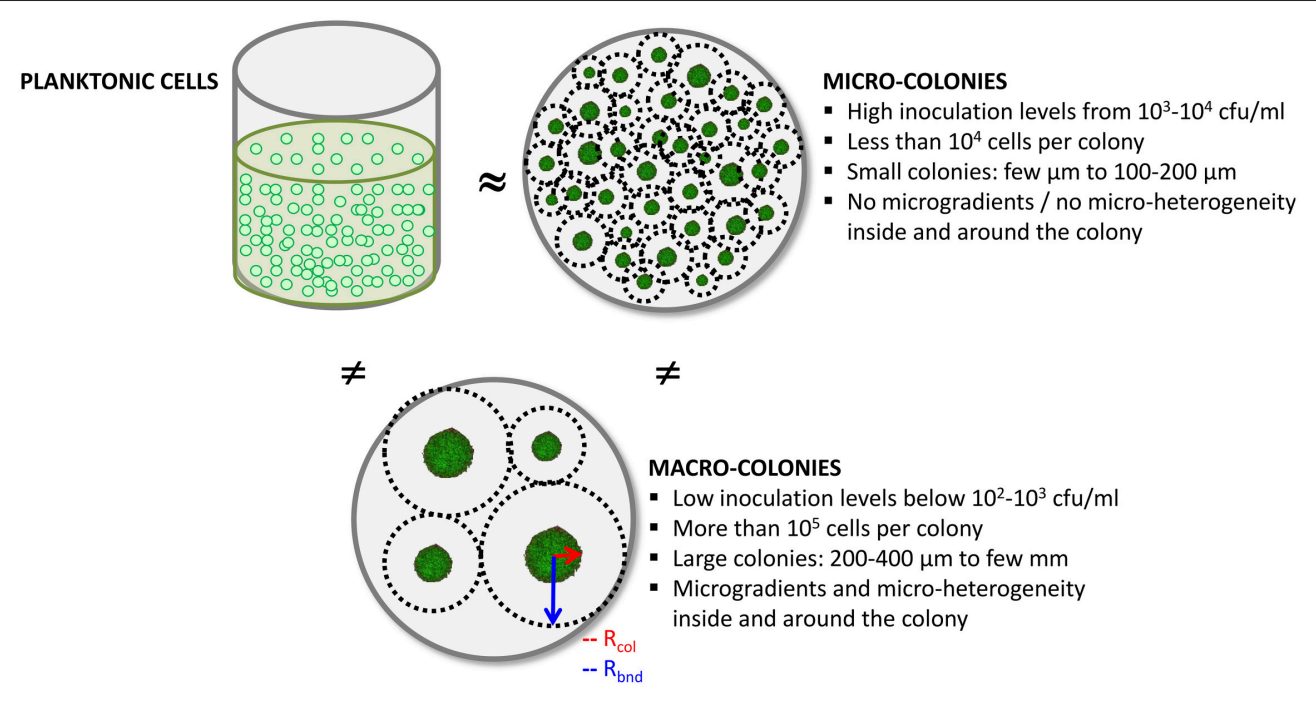

FIGURE 10 | Schematic diagram of the three culture conditions for bacterial cells and their main characteristics; planktonic culture conditions are the most studied.

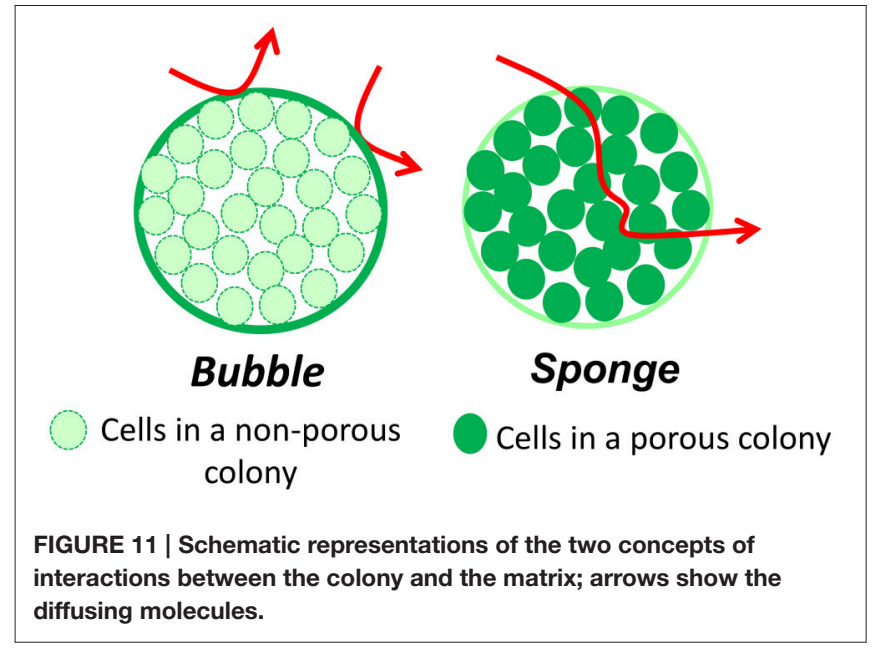

hypotheses lead to the question: are colonies porous to large molecules?

\section{Two Possible Concepts for the Interactions between a Colony and the Surrounding Matrix: "Bubble" or "Sponge"}

We imagined two extreme concepts of the colony (Figure 11): (i) the colony acts as a "bubble" impermeable to molecules and only the periphery cells are in contact with all of the substrates available from the matrix, (ii) or the colony acts as a "sponge" permeable to all the molecules, representing both substrates and end-products which migrate freely through the colony.

If we consider first the "sponge" scenario, the colony is then a group of individual cells all in contact with its microenvironment. The exchange between the micro-environment and the colony is thus that of each of the cells and depends neither on the size of the colony, nor on their number. This concept is close to the planktonic condition in term of interaction of bacteria with the medium. On the contrary, in the "bubble" scenario, the colony can be considered as a tight cluster of cells and only those at the periphery of the colony are in contact with the micro-environment. Thus, for a given number of bacteria, the total exchange area is then determined by the size and the number of colonies, and is of major importance in governing the activity of the colonies within the matrix. The exchange surface (overall exchange surface per unit of medium volume) increases with the number of colonies as their size decreases (Jeanson et al., 2011). The activity of the colonies within the matrix will thus be increased by an increasing exchange surface if the colonies behave as in the "bubble" scenario whereas there will be no effect if colonies behave as in the "sponge" one. As a consequence, in the "bubble" concept, two different inoculation levels will result in two different values for the exchange surface, and thus two different activities for colonies of different sizes containing the same total number of cells. In the case of two different spatial distributions, labeled 1 and 2, the terms $S_{1}$ and $S_{2}$ represent two different exchange surfaces resulting from the two different inoculation levels $I_{1}$ and $I_{2}$. We assume that (i) the packing density of cells and the volume of individual cells inside the colonies are equal for both spatial distributions; (ii) the inoculation level is equal to the number of colonies (one cell gives one colony). Theoretically, for the same final populations, the ratio $S_{1} / S_{2}$ follows the following equation:

$$
\mathrm{S}_{1} / \mathrm{S}_{2}=\left(\mathrm{I}_{1} / \mathrm{I}_{2}\right)^{1 / 3}
$$

However, as seen on Figure 12, the experimental data tend to overestimate the ratio of exchange surfaces $\left(S_{1} / S_{2}\right)$ for a given ratio of inoculation levels $\left(\mathrm{I}_{1} / \mathrm{I}_{2}\right)$ when compared to the theoretical model. The low precision of the experimental measurements may explain this difference. These concepts are 


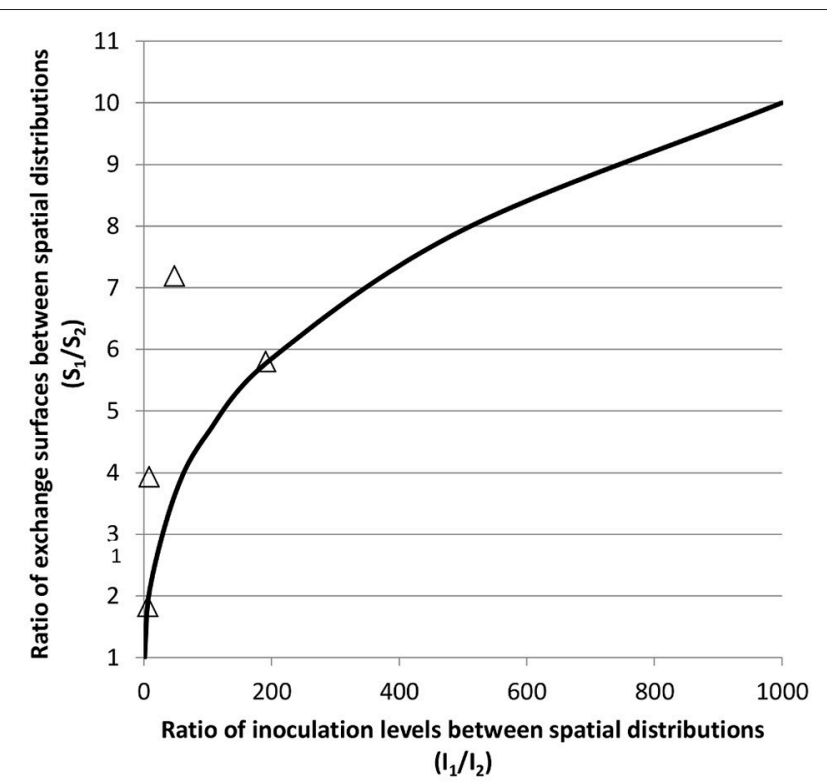

FIGURE 12 | Theoretical relation (black line) for two different spatial distributions, 1 and 2 , between the ratio of the exchange surfaces $\left(S_{1} / S_{2}\right)$ and the ratio of inoculation levels $\left(I_{1} / I_{2}\right) ;(\Delta)$ experimental data either manually measured or obtained from image analysis of confocal microscopy images from Jeanson et al. (2011) and from Le Boucher et al. (2013).

theoretical but may be of great value in food processing. It is thus very important to experimentally explore the question: is the colony functioning as a "bubble" or a "sponge"?

\section{Experimental Exploration of the Two Concepts "Bubble" and "Sponge" The Porosity of Colonies with Respect to Different Types of Molecules}

As described above, milk proteins and dextrans molecules up to $2000 \mathrm{kDa}$ can diffuse within in a model cheese, but are these large molecules able to also diffuse in to the colony?

A first study explored the resistance to diffusion exerted by cells of E. coli and Rhodospirillum rubrum homogeneously immobilized in agar through which solutions of glucose and Lmalic acid could diffuse (Mignot and Junter, 1990). The results showed that the diffusion of glucose and L-malic acid was negatively and linearly correlated with the increase in cell density in the agar, with an increasing resistance to diffusion over the range from $10^{4}$ to $10^{8}$ cells $/ \mathrm{ml}$, probably due to the increased tortuosity imposed by the higher density of cells. Considering the packing density of cells in a colony of about $10^{11}$ cells $/ \mathrm{ml}$ (Malakar et al., 2002a), the resistance to diffusion of molecules within the colony might be expected to be even higher than for a cell density of $10^{8}$ cells $/ \mathrm{ml}$. We thus investigated the porosity of a bacterial colony to molecules of different sizes (Floury et al., 2013). The results showed that dextran molecules from 4 to $155 \mathrm{kDa}$ could penetrate through lactococcal colonies $\left(R_{\text {col }}=15-55 \mu \mathrm{m}\right)$ immobilized in an agar gel and in a model cheese. Indeed, dextran molecules as big as $155 \mathrm{kDa}$ (larger than milk proteins) can diffuse through a bacterial colony but their diffusion coefficient could be limited by their size. On the other hand, milk proteins such as bovine serum albumin, lactoferrin and $\alpha_{s 1}$-casein did not penetrate inside the lactococcal colonies in a model cheese (Floury et al., 2015). We observed the same results with strains of Lactobacillus rhamnosus and L. plantarum (Jeanson, personal communication).

In conclusion, it was clearly demonstrated that the diffusion behavior of macromolecules through bacterial colonies immobilized in a model cheese did not depend so much on the size of the diffusing solute molecules, but mainly on their physicochemical properties (Floury et al., 2015). The colony acts like a "sponge" for the neutral and flexible dextran molecules whilst the colony acts like a "bubble" for all the tested proteins.

\section{Consequences of the Porosity of Bacterial Colonies in Food Fermentation: Example of Cheese}

In cheese, carbon sources such as lactose are soluble and can diffuse freely as in agar or gelatine medium. On the contrary, nitrogen-based substrates are mostly caseins which are bound up in the network and cannot diffuse, except for a minor proportion of free caseins. Assimilable nitrogen substrates are peptides produced from the activity of bacterial cell-wall proteases. In the case of colonies embedded within cheese, only the cells on the periphery can theoretically access the caseins in the network. Taking cheese as an example, this raises the questions: (i) how does the spatial distribution of colonies influence the bacterial metabolism and (ii) how do the cells at the center of the colony access the nitrogen substrates, i.e., the caseins and the caseinderived peptides. If caseins are bound up, one might expect the "bubble" scenario but could the colony act as a "sponge" with respect to the peptides? In order to explore this hypothesis, we measured the influence of two different spatial distributions of micro-colonies of $L$. lactis on the cheese metabolomes during ripening. The inoculations levels, respectively, $1.6 \times 10^{5}$ and $3.1 \times$ $10^{7} \mathrm{cfu} / \mathrm{ml}$ thus $\mathrm{I}_{1} / \mathrm{I}_{2}=191$, generated two sets of model cheeses called small colonies cheeses with $R_{\mathrm{col}}=3.9 \pm 0.2 \mu \mathrm{m}$ and big colonies cheeses with $R_{\text {col }}=26.8 \pm 0.2 \mu \mathrm{m}$ (Le Boucher et al., 2015a). For exactly the same lactococci viable population in the two sets of cheeses, the results showed that lactococci distributed as "small" colonies tended to accelerate proteolysis during ripening in comparison with "big" colonies. As a consequence, small colonies cheeses contained higher amounts of amino acids and some of the peptides than big colonies cheeses. Nevertheless, the increase in concentration of metabolites between small and big colonies cheeses ranged from 1.2 to 2.0 for a ratio of $S_{1} / S_{2}$ equal to 5 (Le Boucher et al., 2015b). Under the hypothesis of a "bubble" scenario, there should have been an increase in the metabolite concentration close to the ratio $S_{1} / S_{2}$, i.e., 5 . Under the hypothesis of a "sponge" scenario, there should not have been observed any changes between the proteolysis of the two different spatial distributions. The results obtained with the small and big colony cheeses are in agreement with the observations on the porosity of colonies. They suggest that the colony acts either as a "sponge" or a "bubble" according to the diffusing molecules, and that some molecules such as peptides may diffuse inside the 
colony reaching the cells located in the center of the colony. The relatively small proportion of cells in the periphery could produce enough peptides for the cells of the whole colony. It has been previously demonstrated for milk that $10 \%$ of a $\mathrm{prt}^{+}$strain of L. lactis could sustain the growth of $90 \%$ of the isogenic prt strain (Juillard and Richard, 1994). Another study performed in a renneted milk gel showed that the overall concentration of total free amino acids was 1.15 times higher than in liquid milk, both being inoculated with a strain of Streptococcus thermophilus at $10^{5} \mathrm{cfu} / \mathrm{ml}$ (Stulova et al., 2015). Even if rennet increased the hydrolysis of caseins into peptides, this result supports the idea that peptides diffuse inside the colony where they are further degraded into amino acids by intracellular aminopeptidases. Furthermore, for inoculation levels from $10^{2}$ to $10^{6} \mathrm{cfu} / \mathrm{ml}$, the growth rates were similar in renneted milk gel and liquid milk during the first exponential phase when the bacteria use the nonprotein nitrogen sources initially present in the milk. However, the growth rates were subsequently lower in the milk gels than in milk during the second exponential phase when the bacterial strain had to synthesize its own cell-wall protease to sustain growth (Stulova et al., 2015). The hypothesis given by the authors was that there was an accumulation of lactic acid around colonies. However, the fact that the total number of bacteria was $13 \%$ higher in the milk gels than in milk at the end of the exponential growth phase, demonstrates that the growth rate was slower but growth lasted longer (Stulova et al., 2015). This result also supports the argument of a limited access to caseins, (leading to the slower growth rate), but to a free access to the peptides.

In conclusion, the interaction of the colony with its surrounding matrix is extremely complex and there are no simple mechanisms that describe how and when the "sponge" and "bubble" conceptions apply. Most likely, the colony acts as a selective filter depending on the properties of the diffusing molecules with a greater preference for flexible and neutral molecules regardless of their size.

\section{CONCLUSIONS}

The objective of this review was a comprehensive understanding based on published literature of the impact of bacterial growth

\section{REFERENCES}

Antwi, M., Bernaerts, K., Van Impe, J. F., and Geeraerd, A. H. (2007). Modelling the combined effects of structured food model system and lactic acid on Listeria innocua and Lactococcus lactis growth in mono- and coculture. Int. J. Food Microbiol. 120, 71-84. doi: 10.1016/j.ijfoodmicro.2007.04.015

Bae, E., Aroonnual, A., Bhunia, A. K., and Hirleman, E. D. (2011a). On the sensitivity of forward scattering patterns from bacterial colonies to media composition. J. Biophotonics 4, 236-243. doi: 10.1002/jbio.201000051

Bae, E., Bai, N., Aroonnual, A., Bhunia, A. K., and Hirleman, E. D. (2011b). Labelfree identification of bacterial microcolonies via elastic scattering. Biotechnol. Bioeng. 108, 637-644. doi: 10.1002/bit.22980

Boons, K., Mertens, L., Van Derlinden, E., David, C. C., Hofkens, J., and Van Impe, J. F. (2013). Behavior of Escherichia coli in a heterogeneous gelatin-dextran mixture. Appl. Environ. Microbiol. 79, 3126-3128. doi: 10.1128/AEM.03782-12

Brocklehurst, T. F., Mitchell, G. A., Ridge, Y. P., Seale, R., and Smith, A. C. (1995). The effect of transient temperatures on the growth of Salmonella typhimurium as colonies in a food context. Overall, the term "bacterial colonies" embrace different situations depending on the spatial distribution of colonies (size and number of colonies) in the matrix. Finally, the spatial distribution emerges as the most crucial parameter in determining whether the immobilization of bacteria has an impact or not. The conclusions differ widely: (i) if colonies are small and numerous (micro-colonies), the implications of growing in colonies rather than as free planktonic growth are minor; (ii) whereas if colonies are large and relatively few in number (macro-colonies), the implications of such immobilization become significant, mostly in terms of a relatively lower growth rates and their lower resistance when under conditions of stress. In the case of bacterial contamination or indigenous microflora, the initial population is low and colonies thus develop as macro-colonies. It is thus important to increase the understanding on the behavior of pathogenic bacteria in solid matrices in order to improve the predictive growth models in solid foods. In the case of LAB in fermented foods, the inoculation levels are high and one can wonder if the growth in micro-colonies really impacts on the growth and the metabolic activity of bacteria in foods by comparison with that as planktonic growth. However, in fermented foods, the interactions between bacterial colonies and the food matrix itself remain unexplained and inadequately studied using agar/gelatine media. Moreover, interactions and even communication between colonies, like quorum sensing, is still unexplored in solid food media (Skandamis and Nychas, 2012). The newly available imaging techniques may open a great field of research in this respect.

\section{AUTHOR CONTRIBUTIONS}

SJ: design and wrote the review manuscript. JF: expert in the diffusion of molecules in cheese and porosity of colonies; improved the review manuscript. VG: expert in proteolysis by bacteria; improved the review manuscript. SL: initiated the topic in the lab; improved the review manuscript. AT: head of the research group; design and extensively improved the review manuscript.

LT2 in gelatin gel. Int. J. Food Microbiol. 27, 45-60. doi: 10.1016/01681605(94)00152-V

Brocklehurst, T. F., Mitchell, G. A., and Smith, A. C. (1997). A model experimental gel-surface for the growth of bacteria on foods. Food Microbiol. 14, 303-311. doi: 10.1006/fmic.1997.0098

Burdikova, Z., Svindrych, Z., Pala, J., Hickey, C. D., Wilkinson, M. G., Panek, J., et al. (2015). Measurement of pH micro-heterogeneity in natural cheese matrices by fluorescence lifetime imaging. Front. Microbiol. 6:183. doi: 10.3389/fmicb.2015.00183

Caldeo, V., and McSweeney, P. L. H. (2012). Changes in oxidationreduction potential during the simulated manufacture of different cheese varieties. Int. Dairy J. 25, 16-20. doi: 10.1016/j.idairyj.2012. 02.002

Choo-Smith, L. P., Maquelin, K., van Vreeswijk, T., Bruining, H. A., Puppels, G. J., Ngo Thi, N. A., et al. (2001). Investigating microbial (micro)colony heterogeneity by vibrational spectroscopy. Appl. Environ. Microbiol. 67, 1461-1469. doi: 10.1128/AEM.67.4.1461-1469.2001 
Cretenet, M., Laroute, V., Ulvé, V., Jeanson, S., Nouaille, S., Even, S., et al. (2011). Dynamic analysis of the Lactococcus lactis transcriptome in cheeses made from milk concentrated by ultrafiltration reveals multiple strategies of adaptation to stresses. Appl. Environ. Microbiol. 77, 247-257. doi: 10.1128/AEM.01174-10

Cussler E. L. (1997). Diffusion: Mass Transfer in Fluid Systems, 2nd Edn. Cambridge University Press. Part of Cambridge Series in Chemical Engineering.

den Besten, H. M. W., Ingham, C. J., van Hylckama Vlieg, J. E. T., Beerthuyzen, M. M., Zwietering, M. H., and Abee, T. (2007). Quantitative analysis of population heterogeneity of the adaptive salt stress response and growth capacity of Bacillus cereus ATCC 14579. Appl. Environ. Microbiol. 73, 4797-4804. doi: 10.1128/AEM.00404-07

Donlan, R. M., and Costerton, J. W. (2002). Biofilms: survival mechanisms of clinically relevant microorganisms. Clin. Microbiol. Rev. 15, 167-193. doi: 10.1128/CMR.15.2.167-193.2002

Drazek, L., Tournoud, M., Derepas, F., Guicherd, M., Mahé, P., Pinston, F., et al. (2015). Three-dimensional characterization of bacterial microcolonies on solid agar-based culture media. J. Microbiol. Meth. 109, 149-156. doi: 10.1016/j.mimet.2014.12.011

Flemming, H. C., and Wingender, J. (2010). The biofilm matrix. Nat. Rev. Microbiol. 8, 623-633. doi: 10.1038/nrmicro2415

Floury, J., El Mourdi, I., Silva, J. V. C., Lortal, S., Thierry, A., and Jeanson, S. (2015). Diffusion of solutes inside bacterial colonies immobilized in model cheese depends on their physicochemical properties: a time-lapse microscopy study. Front. Microbiol. 6:366. doi: 10.3389/fmicb.2015.00366

Floury, J., Jeanson, S., Aly, S., and Lortal, S. (2010). Determination of the diffusion coefficients of small solutes in cheese: a review. Dairy Sci. Technol. 90, 477-508. doi: $10.1051 / \mathrm{dst} / 2010011$

Floury, J., Jeanson, S., Madec, M. N., and Lortal, S. (2013). Porosity of Lactococcus lactis subsp. lactis LD61 colonies immobilised in model cheese. Int. J. Food Microbiol. 163, 64-70. doi: 10.1016/j.ijfoodmicro.2013.02.014

Floury, J., Madec, M. N., Waharte, F., Jeanson, S., and Lortal, S. (2012). First assessment of diffusion coefficients in model cheese by fluorescence recovery after photobleaching (FRAP). Food Chem. 133, 551-556. doi: 10.1016/j.foodchem.2012.01.030

Gonzalez, D. J., Xu, Y., Yang, Y. L., Esquenazi, E., Liu, W. T., Edlund, A., et al. (2012). Observing the invisible through imaging mass spectrometry, a window into the metabolic exchange patterns of microbes. J. Proteomics 75, 5069-5076. doi: 10.1016/j.jprot.2012.05.036

Guillier, L., Pardon, P., and Augustin, J. C. (2006). Automated image analysis of bacterial colony growth as a tool to study individual lag time distributions of immobilized cells. J. Microbiol. Meth. 65, 324-334. doi: 10.1016/j.mimet.2005.08.007

Hickey, C. D., Sheehan, J. J., Wilkinson, M. G., and Auty, M. A. E. (2015). Growth and location of bacterial colonies within dairy foods using microscopy techniques: a review. Front. Microbiol. 6:99. doi: 10.3389/fmicb.2015.00099

Hills, B. P. (2001). “The power and pitfalls of deductive modelling," in Food Process Modelling, eds L. M. M. Tijskens, M. L. A. T. M. Hertog, and B. M.Nicolaï (Cambridge: Woodhead Publishing Limited), 3-17.

Ingham, C. J., Beerthuyzen, M., and van Hylckama Vlieg, J. (2008). Population heterogeneity of Lactobacillus plantarum WCFS1 microcolonies in response to and recovery from acid stress. Appl. Environ. Microbiol. 74, 7750-7758. doi: 10.1128/AEM.00982-08

Jeanson, S., Chadoeuf, J., Madec, M.-N., Aly, S., Floury, J., Brocklehurst, T. F., et al. (2011). Spatial distribution of bacterial colonies in a model cheese. Appl. Environ. Microbiol. 77, 1493-1500. doi: 10.1128/AEM.02233-10

Jeanson, S., Floury, J., Issulahi, A. A., Madec, M. N., Thierry, A., and Lortal, S. (2013). Microgradients of $\mathrm{pH}$ do not occur around Lactococcus colonies in a model cheese. Appl. Environ. Microbiol. 79, 6516-6518. doi: 10.1128/AEM.01678-13

Juillard, V., and Richard, J. (1994). Mixed cultures in milk of a proteinase-positive and a proteinase-negative variant of Lactococcus lactis subsp lactis: influence of initial percentage of proteinase-positive cells on the growth parameters of each strain and on the rate of acidification. Lait 74, 3-12. doi: 10.1051/lait:199411

Kabanova, N., Stulova, I., and Vilu, R. (2012). Microcalorimetric study of the growth of bacterial colonies of Lactococcus lactis IL1403 in agar gels. Food Microbiol. 29, 67-79. doi: 10.1016/j.fm.2011.08.018

Kabanova, N., Stulova, I., and Vilu, R. (2013). Microcalorimetric study of growth of Lactococcus lactis IL1403 at low glucose concentration in liquids and solid agar gels. Thermochim. Acta 559, 69-75. doi: 10.1016/j.tca.2013.02.013
Kim, H., Singh, A. K., Bhunia, A. K., and Bae, E. (2014). Laser-induced speckle scatter patterns in Bacillus colonies. Front. Microbiol. 5:537. doi: 10.3389/fmicb.2014.00537

Knudsen, G. M., Nielsen, M. B., Grassby, T., Danino-Appleton, V., Thomsen, L. E., Colquhoun, I. J., et al. (2012). A third mode of surface-associated growth: immobilization of Salmonella enterica serovar Typhimurium modulates the RpoS-directed transcriptional programme. Environ. Microbiol. 14, 1855-1875. doi: 10.1111/j.1462-2920.2012.02703.x

Koutsoumanis, K. P., Kendall, P. A., and Sofos, J. N. (2004). A comparative study on growth limits of Listeria monocytogenes as affected by temperature, $\mathrm{pH}$ and aw when grown in suspension or on a solid surface. Food Microbiol. 21, 415-422. doi: 10.1016/j.fm.2003.11.003

Koutsoumanis, K. P., and Lianou, A. (2013). Stochasticity in colonial growth dynamics of individual bacterial cells. Appl. Environ. Microbiol. 79, 2294-2301. doi: 10.1128/AEM.03629-12

Kreft, J. U., Booth, G., and Wimpenny, J. W. T. (1998). BacSim, a simulator for individual-based modelling of bacterial colony growth. Microbiology 144, 3275-3287. doi: 10.1099/00221287-144-12-3275

Kristoffersen, T. (1985). Development of flavor in cheese. Milchwissenschaft 40, 197-199.

Le Boucher, C., Courant, F., Jeanson, S., Chereau, S., Maillard, M. B., Royer, A. L., et al. (2013). First mass spectrometry metabolic fingerprinting of bacterial metabolism in a model cheese. Food Chem. 141, 1032-1040. doi: 10.1016/j.foodchem.2013.03.094

Le Boucher, C., Courant, F., Royer, A.-L., Jeanson, S., Lortal, S., Dervilly-Pinel, G. et al. (2015a). LC-HRMS fingerprinting as an efficient approach to highlight fine differences in cheese metabolome during ripening. Metabolomics 11, 1117-1130. doi: 10.1007/s11306-014-0769-0

Le Boucher, C., Gagnaire, V., Briard-Bion, V., Jardin, J., Maillard, M.-B., DervillyPinel, G., et al. (2015b). The spatial distribution of Lactococcus lactis colonies modulates the production of major metabolites during the ripening of a model cheese. Appl. Environ. Microbiol. doi: 10.1128/AEM.02621-15. [Epub ahead of print].

Lobete, M. M., Fernandez, E. N., and Van Impe, J. F. M. (2015). Recent trends in non-invasive in situ techniques to monitor bacterial colonies in solid (model) food. Front. Microbiol. 6:148. doi: 10.3389/fmicb.2015.00148

Malakar, P. K., Barker, G. C., Zwietering, M. H., and van't Riet, K. (2003). Relevance of microbial interactions to predictive microbiology. Int. J. Food Microbiol. 84, 263-272. doi: 10.1016/S0168-1605(02)00424-5

Malakar, P. K., Brocklehurst, T. F., MacKie, A. R., Wilson, P. D. G., Zwietering, M. H., and van't Riet, K. (2000). Microgradients in bacterial colonies: use of fluorescence ratio imaging, a non-invasive technique. Int. J. Food Microbiol. 56, 71-80. doi: 10.1016/S0168-1605(00)00222-1

Malakar, P. K., Martens, D. E., van Breukelen, W., Boom, R. M., Zwietering, M. H., and van 't Riet, K. (2002a). Modeling the interactions of Lactobacillus curvatus colonies in solid medium: consequences for Food Quality and Safety. Appl. Environ. Microbiol. 68, 3432-3441. doi: 10.1128/AEM.68.7.3432-3441.2002

Malakar, P. K., Zwietering, M. H., Boom, R. M., Brocklehurst, T. F., Wilson, P. D., MacKie, A. R., et al. (2002b). Diffusion of lactic acid in a buffered gel system supporting growth of Lactobacillus curvatus. J. Sci. Food Agric. 82, 1729-1734. doi: $10.1002 /$ jsfa.1256

McKay, A. L., and Peters, A. C. (1995). The effect of sodium chloride concentration and $\mathrm{pH}$ on the growth of Salmonella typhimurium colonies on solid medium. J. Appl. Bacteriol. 79, 353-359. doi: 10.1111/j.1365-2672.1995.tb03148.x

McKay, A. L., Peters, A. C., and Wimpenny, J. W. T. (1997). Determining specific growth rates in different regions of Salmonella typhimurium colonies. Lett. Appl. Microbiol. 24, 74-76. doi: 10.1046/j.1472-765X.1997.00354.x

Meldrum, R. J., Brocklehurst, T. F., Wilson, D. R., and Wilson, P. D. G. (2003). The effects of cell immobilization, $\mathrm{pH}$ and sucrose on the growth of Listeria monocytogenes Scott A at $10^{\circ}$ C. Food Microbiol. 20, 97-103. doi: 10.1016/S0740-0020(02)00083-7

Mertens, L., Van Derlinden, E., and Van Impe, J. F. (2012). A novel method for high-throughput data collection in predictive microbiology: optical density monitoring of colony growth as a function of time. Food Microbiol. 32, 196-201. doi: 10.1016/j.fm.2012.04.001

Mignot, L., and Junter, G. A. (1990). Diffusion in immobilized-cell agar layers: influence of microbial burden and cell morphology on the diffusion coefficients of L-malic acid and glucose. Appl. Microbiol. Biotechnol. 32, 418-423. doi: 10.1007/BF00903776 
Mikkelsen, H., Duck, Z., Lilley, K. S., and Welch, M. (2007). Interrelationships between colonies, biofilms, and planktonic cells of Pseudomonas aeruginosa. J. Appl. Bacteriol. 189, 2411-2416. doi: 10.1128/JB.01687-06

Ngo Thi, N. A., and Naumann, D. (2007). Investigating the heterogeneity of cell growth in microbial colonies by FTIR microspectroscopy. Anal. Bioanal. Chem. 387, 1769-1777. doi: 10.1007/s00216-006-0829-z

Ngo-Thi, N., Kirschner, C., and Naumann, D. (2003). Characterization and identification of microorganisms by FT-IR microspectrometry. J. Mol. Struct. 661-662, 371-380. doi: 10.1016/j.molstruc.2003.08.012

Nielsen, M. B., Knudsen, G. M., Danino-Appleton, V., Olsen, J. E., and Thomsen, L. E. (2013). Comparison of heat stress responses of immobilized and planktonic Salmonella enterica serovar Typhimurium. Food Microbiol. 33, 221-227. doi: 10.1016/j.fm.2012.09.020

Øyaas, J., Storrø, I., Svendsen, H., and Levine, D. W. (1995). The effective diffusion coefficient and the distribution constant for small molecules in calcium-alginate gel beads. Biotechnol. Bioeng. 47, 492-500. doi: 10.1002/bit.260470411

Parker, M. L., Gunning, P. A., Macedo, A. C., Malcata, F. X., and Brocklehurst, T. F. (1998). The microstructure and distribution of micro-organisms within mature Serra cheese. J. Appl. Microbiol. 84, 523-530. doi: 10.1046/j.13652672.1998.00375.x

Pipe, L. Z., and Grimson, M. J. (2008). Spatial-temporal modelling of bacterial colony growth on solid media. Mol. Biosyst. 4, 192-198. doi: 10.1039/b708241j

Pirt, S. J. (1967). A kinetic study of the mode of growth of surface colonies of bacteria and fungi. J. Gen. Microbiol. 47, 181-197. doi: 10.1099/00221287-472-181

Reyrolle, J., and Letellier, F. (1979). Autoradiographic study of the localization and evolution of growth zones in bacterial colonies. J. Gen. Microbiol. 111, 399-406. doi: 10.1099/00221287-111-2-399

Ribeiro, A. C. F., Lobo, V. M. M., Leaist, D. G., Natividade, J. J. S., Veríssimo, L. P., Barros, M. C. F., et al. (2005). Binary diffusion coefficients for aqueous solutions of lactic acid. J. Solution Chem. 34, 1009-1016. doi: 10.1007/s10953-005-6987-3

Ryssel, M., Duan, Z., and Siegumfeldt, H. (2013). In situ examination of cell growth and death of Lactococcus lactis. FEMS Microbiol. Lett. 343, 82-88. doi: 10.1111/1574-6968.12134

Shapiro, J. A. (1987). Organization of developing Escherichia coli colonies viewed by scanning electron microscopy. J. Bacteriol. 169, 142-156.

Silva, J. V. C., Peixoto, P., Lortal, S., and Floury, J. (2013). Transport phenomena in a model cheese: the influence of the charge and the shape of solutes on diffusion. J. Dairy Sci. 96, 6186-6198. doi: 10.3168/jds.2013-6552

Skandamis, P. N., Brocklehurst, T. F., Panagou, E. Z., and Nychas, G. -J. E. (2007). Image analysis as a mean to model growth of Escherichia coli O157:H7 in gel cassettes. J. Appl. Microbiol. 103, 937-947. doi: 10.1111/j.13652672.2007.03358.x

Skandamis, P. N., and Jeanson, S. (2015). Colonial vs planktonic type of growth: mathematical modeling of microbial dynamics on surfaces and in liquid, semiliquid and solid foods. Front. Microbiol. 6:1178. doi: 10.3389/fmicb.2015.01178

Skandamis, P. N., and Nychas, G. J. (2012). Quorum sensing in the context of food microbiology. Appl. Environ. Microbiol. 78, 5473-5482. doi: 10.1128/AEM.00468-12

Skandamis, P., Tsigarida, E., and Nychas, G. (2000). Ecophysiological attributes of Salmonella typhimurium in liquid culture and within a gelatin gel with or without the addition of oregano essential oil. World J. Microbiol. Biotechnol. 16, 31-35. doi: 10.1023/A:1008934020409

Stecchini, M. L., Del Torre, M., Sarais, I., Saro, O., Messina, M., and Maltini, E. (1998). Influence of structural properties and kinetic constraints on Bacillus cereus growth. Appl. Environ. Microbiol. 64, 1075-1078.

Stulova, I., Kabanova, N., Krišciunaite, T., Adamberg, K., Laht, T. M., and Vilu, R. (2015). Microcalorimetric study of the growth of Streptococcus thermophilus in renneted milk. Front. Microbiol. 6:79. doi: 10.3389/fmicb.2015.00079

Tammam, J. D., Williams, A. G., Banks, J., Cowie, G., and Lloyd, D. (2001). Membrane inlet mass spectrometric measurement of $\mathrm{O}_{2}$ and $\mathrm{CO}_{2}$ gradients in cultures of Lactobacillus paracasei and a developing Cheddar cheese ecosystem. Int. J. Food Microbiol. 65, 11-22. doi: 10.1016/S0168-1605(00)00438-4

Theys, T. E., Geeraerd, A. H., Devlieghere, F., and Van Impe, J. F. (2009b). Extracting information on the evolution of living- and dead-cell fractions of Salmonella Typhimurium colonies in gelatin gels based on microscopic images and plate-count data. Lett. Appl. Microbiol. 49, 39-45. doi: 10.1111/j.1472765X.2009.02623.x
Theys, T. E., Geeraerd, A. H., Devlieghere, F., and Van Impe, J. F. (2010). On the selection of relevant environmental factors to predict microbial dynamics in solidified media. Food Microbiol. 27, 220-228. doi: 10.1016/j.fm.2009.10.005

Theys, T. E., Geeraerd, A. H., and Van Impe, J. F. (2009a). Evaluation of a mathematical model structure describing the effect of (gel) structure on the growth of Listeria innocua, Lactococcus lactis and Salmonella Typhimurium. J. Appl. Microbiol. 107, 775-784. doi: 10.1111/j.1365-2672.2009.04256.x

Theys, T. E., Geeraerd, A. H., Verhulst, A., Poot, K., van Bree, I., Devlieghere, F., et al. (2008). Effect of $\mathrm{pH}$, water activity and gel micro-structure, including oxygen profiles and rheological characterization, on the growth kinetics of Salmonella Typhimurium. Int. J. Food Microbiol. 128, 67-77. doi: 10.1016/j.ijfoodmicro.2008.06.031

Thomas, L. V., and Wimpenny, J. W. (1996a). Investigation of the effect of combined variations in temperature, $\mathrm{pH}$, and $\mathrm{NaCl}$ concentration on nisin inhibition of Listeria monocytogenes and Staphylococcus aureus. Appl. Environ. Microbiol. 62, 2006-2012.

Thomas, L. V., and Wimpenny, J. W. T. (1996b). Competition between Salmonella and Pseudomonas species growing in and on agar, as affected by $\mathrm{pH}$, sodium chloride concentration and temperature. Int. J. Food Microbiol. 29, 361-370. doi: 10.1016/0168-1605(95)00077-1

Thomas, L. V., Wimpenny, J. W. T., and Barker, G. C. (1997). Spatial interactions between subsurface bacterial colonies in a model system: a territory model describing the inhibition of Listeria monocytogenes by a nisin-producing lactic acid bacterium. Microbiology 143, 2575-2582. doi: 10.1099/00221287-143-82575

Vilain, S., Cosette, P., Hubert, M., Lange, C., Junter, G. A., and Jouenne, T. (2014). Comparative proteomic analysis of planktonic and immobilized Pseudomonas aeruginosa cells: a multivariate statistical approach. Anal. Biochem. 329, 120-130. doi: 10.1016/j.ab.2004.02.014

Walker, S. L., Brocklehurst, T. F., and Wimpenny, J. W. T. (1998). Adenylates and adenylate-energy charge in submerged and planktonic cultures of Salmonella enteritidis and Salmonella typhimurium. Int. J. Food Microbiol. 44, 107-113. doi: 10.1016/S0168-1605(98)00126-3

Walker, S. L., Brocklehurst, T. F., and Wimpenny, J. W. T. (1997). The effects of growth dynamics upon $\mathrm{pH}$ gradient formation within and around subsurface colonies of Salmonella typhimurium. J. Appl. Microbiol. 82, 610-614. doi: 10.1111/j.1365-2672.1997.tb02869.x

Wilson, P. D. G., Brocklehurst, T. F., Arino, S., Thuault, D., Jakobsen, M., Lange, M., et al. (2002). Modelling microbial growth in structured foods: towards a unified approach. Int. J. Food Microbiol. 73, 275-289. doi: 10.1016/S01681605(01)00660-2

Wimpenny, J. W. (1992). "Microbial systems-patterns in time and space," in Advances in Microbial Ecology, ed K. C. Marshall (New York, NY: Plenum Press), 469-522.

Wimpenny, J. W. T., Leistner, L., Thomas, L. V., Mitchell, A. J., Katsaras, K., and Peetz, P. (1995). Submerged bacterial colonies within food and model systems: their growth, distribution and interactions. Int. J. Food Microbiol. 28, 299-315. doi: 10.1016/0168-1605(95)00065-8

Wright, K. M., Coleman, H. P., MacKie, A. R., Parker, M. L., Brocklehurst, T. F., Wilson, D. R., et al. (2000). Determination of mean growth parameters of bacterial colonies immobilized in gelatin gel using a laser gel-cassette scanner. Int. J. Food Microbiol. 57, 75-89. doi: 10.1016/S0168-1605(00) 00229-4

Zhao, Y., Knøchel, S., and Siegumfeldt, H. (2014). In situ examination of Lactobacillus brevis after exposure to an oxidizing disinfectant. Front. Microbiol. 5:623. doi: 10.3389/fmicb.2014.00623

Conflict of Interest Statement: The authors declare that the research was conducted in the absence of any commercial or financial relationships that could be construed as a potential conflict of interest.

Copyright (C) 2015 Jeanson, Floury, Gagnaire, Lortal and Thierry. This is an openaccess article distributed under the terms of the Creative Commons Attribution License (CC BY). The use, distribution or reproduction in other forums is permitted, provided the original author(s) or licensor are credited and that the original publication in this journal is cited, in accordance with accepted academic practice. No use, distribution or reproduction is permitted which does not comply with these terms. 


\section{OPEN ACCESS}

Edited by:

Jean-christophe Augustin, Ecole Nationale Vétérinaire d'Alfort,

France

Reviewed by:

Laurent Guillier,

Agence Nationale de Sécurité

Sanitaire de l'Alimentation, de l' Environnement et du Travail, France Juan Aguirre, Universidad Complutense, Madrid, Spain

${ }^{*}$ Correspondence: Panagiotis N. Skandamis pskan@aua.gr

Specialty section:

This article was submitted to Food Microbiology,

a section of the journal

Frontiers in Microbiology

Received: 09 July 2015 Accepted: 12 October 2015 Published: 29 October 2015

Citation: Skandamis PN and Jeanson S (2015) Colonial vs. planktonic type of growth: mathematical modeling of microbial dynamics on surfaces and in liquid, semi-liquid and solid foods.

Front. Microbiol. 6:1178. doi: 10.3389/fmicb.2015.01178

\section{Colonial vs. planktonic type of growth: mathematical modeling of microbial dynamics on surfaces and in liquid, semi-liquid and solid foods}

\author{
Panagiotis N. Skandamis ${ }^{1 *}$ and Sophie Jeanson ${ }^{2,3}$ \\ ${ }^{1}$ Laboratory of Food Quality Control and Hygiene, Department of Food Science and Human Nutrition, University of Athens, \\ Athens, Greece, ${ }^{2}$ Institut National de la Recherche Agronomique, UMR1253 Science and Technology of Milk and Eggs, \\ Rennes, France, ${ }^{3}$ AGROCAMPUS OUEST, UMR1253 Science and Technology of Milk and Eggs, Rennes, France
}

Predictive models are mathematical expressions that describe the growth, survival, inactivation, or biochemical processes of foodborne bacteria. During processing of contaminated raw materials and food preparation, bacteria are entrapped into the food residues, potentially transferred to the equipment surfaces (abiotic or inert surfaces) or cross-contaminate other foods (biotic surfaces). Growth of bacterial cells can either occur planktonically in liquid or immobilized as colonies. Colonies are on the surface or confined in the interior (submerged colonies) of structured foods. For low initial levels of bacterial population leading to large colonies, the immobilized growth differs from planktonic growth due to physical constrains and to diffusion limitations within the structured foods. Indeed, cells in colonies experience substrate starvation and/or stresses from the accumulation of toxic metabolites such as lactic acid. Furthermore, the micro-architecture of foods also influences the rate and extent of growth. The micro-architecture is determined by (i) the non-aqueous phase with the distribution and size of oil particles and the pore size of the network when proteins or gelling agent are solidified, and by (ii) the available aqueous phase within which bacteria may swarm or swim. As a consequence, the micro-environment of bacterial cells when they grow in colonies might greatly differs from that when they grow planktonically. The broth-based data used for modeling (lag time and generation time, the growth rate, and population level) are poorly transferable to solid foods. It may lead to an over-estimation or under-estimation of the predicted population compared to the observed population in food. If the growth prediction concerns pathogen bacteria, it is a major importance for the safety of foods to improve the knowledge on immobilized growth. In this review, the different types of models are presented taking into account the stochastic behavior of single cells in the growth of a bacterial population. Finally, the recent advances in the rules controlling different modes of growth, as well as the methodological approaches for monitoring and modeling such growth are detailed.

Keywords: bacterial colony, stochastic, stress response, lag time, microstructure, micro-environment 


\section{INTRODUCTION TO PREDICTIVE MICROBIOLOGY}

Predictive food microbiology is a sub-discipline of food microbiology that uses models (i.e., mathematical equations) to describe the growth, survival, or inactivation of microbes in food systems. Mathematical models refer to a set of basic hypotheses supporting the target (bio-) processes which are to be simulated and are possibly algebraic functions and/or differential equations (Baranyi and Roberts, 1995). Therefore, with predictive microbiology, all the knowledge of microbial responses in different environmental conditions is summarized as mathematical equations. McMeekin et al. (2008), stated that "the model is often a simplified description of relationships between observations of the system (responses) and the factors that are believed to cause the observed responses."

The long roadmap of predictive microbiology over the last four decades, along with the advances toward understanding and quantifying microbial responses down to single cell level, have led to the appointment of predictive modeling as one of the most promising decision-support methodologies for food safety assessment by the Food Industry and competent authorities. Predictive modeling has been greatly benefitted by the technological and scientific evolution in both collection and processing of data, through the introduction of-omics (Rantsiou et al., 2011; Brul et al., 2012), the deployment of advanced microscopy techniques (e.g., confocal laser microscopy and fluorophores fused in the genome; Locke and Elowitz, 2009; Cox et al., 2010; Bridier et al., 2015), the application of chemometrics, data mining and the emergence of advanced data modeling techniques (e.g., artificial neural networks; Argyri et al., 2010; Panagou et al., 2011). As a next step, the rising trend for application of predictive modeling in daily practice has intensified the need to systematically exploit the vast number of available predictive models so far. Meeting this demand is being markedly achieved through the development of collective predictive modeling repositories (e.g., ComBase, Pathogen Modeling Program, iRisk, Food Spoilage and Safety Predictor, Sym'Previus, etc.). They are equipped with search engines for guided-retrieval of the appropriate food-specific or generic models (i.e., not food-specific) associated with particular hazards and built-in fitting or simulation modules, in order to visualize and numerically express the model outputs in comprehensive and ready to use formats. Such a variety of predictive models and risk assessment/risk ranking software tools may indeed help the food producers, researchers and food safety inspectors to apply the concepts of predictive modeling in quality-by-design, identification of safe product formulations and evaluation of products compliance with safety standards and microbiological criteria. A comprehensive and quite extensive review of the available software tools can be found in the study by Tenenhaus-Aziza and Ellouze (2014).

Models alone should be applied in caution and with proper disclaimers in the context of decision-making during Hazard Analysis Critical Control Point (HACCP) plan development. Use of models requires experience and judgment, both in modeling and food microbiology. Therefore, it is of vital importance to clearly perceive that the predictive models and associated software tools should not replace the expert opinion, but rather assist the experts (and sometimes even the non-experts) to elicit a food safety plan (Tenenhaus-Aziza and Ellouze, 2014). When models alone are used to make a decision, those models must be shown to be valid for the food in question and should take into consideration lot-to-lot variation. Validation may be based on published or unpublished data for very similar or identical foods. Nonetheless, even in cases when the available predictions are obtained from lab-media based models, which may potentially overlook some significant food-specific impacts on microbial behavior, such predictions are still very useful in guiding more focused and targeted challenge testing (Baranyi and Roberts, 1995; McDonald and Sun, 1999; McMeekin et al., 2008).

\section{MODEL TYPES AND CLASSIFICATION}

Based on microbial responses, expressed as change in numbers and stress tolerance, the combinations of intrinsic and extrinsic environmental determinants to which microorganisms may be exposed, are divided into the following major domains: the growth era and the domain including the combinations that allow survival or cause death of microorganisms (Booth, 2002). The conditions that lie between these two domains refer to a zone where microbial responses are uncertain and characterized by the growth/no growth interface (Le Marc et al., 2005). This zone is strongly associated with the so-called cardinal values $\left(T, \mathrm{pH}, \mathrm{a}_{\mathrm{w}}\right.$, etc.) for growth and outlines the bio-kinetic range of microbial proliferation. Such values are species- or even strain-dependent and thus, introduce significant variability in the assessment of the impact of marginal growth conditions on microbial growth, an issue commonly encountered in quantitative microbial risk assessment. To remedy that, models have been proposed which embed the theoretical growth-limiting values for critical hurdles, such as temperature, $\mathrm{a}_{\mathrm{w}}, \mathrm{pH}, \mathrm{CO}_{2}$ and preservatives as biological meaningful parameters in the model structure. Notably, a theoretical interface also exists between survival and inactivation separating combinations that cause growth cessation but not cellular death from those that are lethal (McKellar et al., 2002).

Depending on the conceptual modeling approach applied to the target biochemical process and the final algebraic form, the models can be characterized as empirical or phenomenological, which mathematically describe specific behavior, and mechanistic or theoretical models with a biological basis, which search for the underlying mechanisms driving already observed phenomena. Polynomial equations are the most common empirical models. These models are easy to use, straightforward and no knowledge of a particular process is required. However, polynomial models have no theoretical foundation and have numerous parameters without biological meaning. Therefore, they do not offer any knowledge to mechanisms underlying a process. Polynomial models are commonly represented as quadratic response surfaces describing the environment dependence of a parameter of a bacterial population (Gibson et al., 1988).

Based on the type of dependent variable that is predicted, the models can be classified as kinetic or probabilistic. Kinetic 
models predict the extent and rate of growth or inactivation of a microorganism. The growth rate of a microorganism can be modeled in order to be used for making predictions based on the exponential growth of the corresponding microbial population. Kinetic models can be used to predict changes in microbial numbers with time, even if a controlling variable, which can affect growth, is changing (McDonald and Sun, 1999). This type of models constitutes a fundamental model category in predictive microbiology, especially for ready-to-eat foods, since they may assess the exposure of consumers to the doses (levels) of pathogenic bacteria at the time of consumption. The purpose of kinetic models is to estimate the time required for a specified growth or inactivation response to occur under certain intrinsic or extrinsic conditions. Such conditions include temperature, $\mathrm{pH}, \mathrm{a}_{\mathrm{w}}$, packaging atmosphere (e.g., $\mathrm{CO}_{2}$ levels), redox potential (Eh), the rheological properties of the food (structure-associated variables), relative humidity, nutrient content and the concentration of antimicrobial compounds (Theys et al., 2009b; Mejlholm et al., 2010; Møller et al., 2013). Thermal inactivation was the first microbial inactivation process modeled since 1920 by the canned food industry, in order to control the risk of Clostridium botulinum toxigenesis. First-order inactivation models were used to describe a log-linear trend of C. botulinum spores in low acid canned food. Through the slope of inactivation curves the thermal death time was estimated and particularly in low acid canned foods, a 12-decimal reduction (12D) of C. botulinum spores was shown to require exposure to $121^{\circ} \mathrm{C} / 15$ psi for $15 \mathrm{~min}$. Over the last decades the microbial inactivation modeling was expanded to account for nonthermal inactivation (Buchanan et al., 1997). In addition to the classical linear inactivation curve, the concept of biphasic death, associated with the pre-existence or emergence of a resistant sub-populations throughout exposure to lethal conditions was modeled with non-linear models (Whiting, 1993; Geeraerd et al., 2005). Probabilistic models constitute the corner stone of predicting microbial dynamics, acting as the filter, i.e., likelihoodbased decision of the primary microbial response (growth or inactivation) and guiding the selection of the subsequent kinetic modeling tool, i.e., growth or inactivation model, for predicting the change in microbial numbers in time. As such, the fate of microbial populations in foods is eventually dependent on the probability of growth or inactivation phenomena defined by the intrinsic and extrinsic factors of foods and processing environment. From a closer perspective, the behavior of an isogenic (homogeneous) population is the cumulative and stochastic outcome of its individual cells (microscopic level; Kutalik et al., 2005). Each cell within a microbial population is characterized by a variable probability for growth initiation (Koutsoumanis, 2008), followed by a stochastically defined lag time, i.e., sampled from a probability distribution (Francois et al., 2005, 2006a, 2007; Guillier et al., 2005, 2006), both resulting in a fractional growth of the total population with various subpopulations (mesoscopic level) initiating growth on different times (McKellar and Knight, 2000; McKellar, 2001). It has been suggested that under given conditions the geometric lag, i.e., the intersection of the slope at exponential phase with horizontal asymptote at the initial population level, is essentially dependent on the cumulative behavior of the fraction(s) of the initial population, which either possesses the shortest lag time (i.e., the earliest growth starters), and/or the fastest generation time (McKellar and Knight, 2000; Koutsoumanis, 2008). As a mirror image, under lethal conditions, e.g., $\mathrm{pH}<3.0$, or $\mathrm{T}>60^{\circ} \mathrm{C}$, the inactivation curve of a microbial population, represented by a curve of survivors (\%) vs. time, is the result of the cumulative distribution of the individual cell death time, i.e., the time required to kill every single cell (Aspridou and Koutsoumanis, 2014). In explicit terms, probability models can be used to predict the likelihood of the occurrence of a microbial response as a function of intrinsic and extrinsic factors of foods and processing environment (Ross and Dalgaard, 2004). Microbial responses which have been modeled with this approach include spore germination, toxin formation by C. botulinum, growth initiation and survival or death of bacteria as a result of lethal $\mathrm{pH}$ and organic acid combinations. In the context of industrial practice, such models together with cardinal growth models may be of great assistance to HACCP, by offering science-based numerical evidence for setting critical limits, establishing process or product criteria and assessing the compliance of a given process to these limits or the legislative microbiological criteria (e.g., EC Regulation 2073/2005).

All the above model types may be further divided into the following categories, based on the combination of dependent (predicted) and independent (explanatory) variable (Whiting and Buchanan, 1993; McDonald and Sun, 1999):

i. the primary models, which are used to describe the changes of the microbial population density as a function of time using a limited number of kinetic parameters (e.g., lag time, growth or inactivation rate and maximum population density);

ii. the secondary models expressing the effect of environmental variables (e.g., temperature, $\mathrm{NaCl}, \mathrm{pH}$, etc.) on the kinetic parameters estimated by the primary models;

iii. the tertiary models, which are computer tools that integrate the primary and secondary models into user-friendly units. The wider use of models in the food industry and research depends on the availability of user-friendly software (Psomas et al., 2011; http://www.aua.gr/psomas/ gropin/), which encompass predictive models and allow different users to retrieve information from them in a rapid and convenient way (McMeekin et al., 2008, 2013).

The impact on microbial growth of the aforementioned intrinsic and extrinsic variables described by the models is strongly dependent on the structure of food or the model substrate. Based on that, in the following lines, a review is performed of existing modeling approaches accounting for different forms of microbial growth on surfaces, or in the interior of food matrices, either in suspension or immobilized in colonies.

\section{Growth Rate of Microorganisms in Different Forms of Growth}

In foods, microbial growth occurs in the aqueous phase. The structural characteristics (e.g., viscosity, 3D structured grid, also called "micro-architecture") of this phase, resulting from 
hydrophilic structure-inducing agents, in combination with the total concentration and dispersion of water compared to fat phase determine the form and rate of growth, i.e., the spatiotemporal microbial dynamics. Food may be characterized as liquid (e.g., juices), gelled (e.g., jellies, cottage, marmelades), oil-in-water emulsions (e.g., mayonnaise, milk), or water-in-oil emulsions (e.g., butter and margarine) and the composite form of gelled emulsions (i.e., an immobilized oil-in-water emulsion). The type of emulsion determines the distribution of available water (Møller et al., 2013). The growth rate of microorganisms in response to food structure, for a given set of intrinsic and extrinsic parameters, is dependent on the motility of cells in the aqueous phase, the extent of resulting (micro-)colony immobilization and the diffusion kinetics of nutrient, oxygen, and metabolites. Three different status of growth may occur depending on the food structure:

(1) If bacteria are suspended in liquids, their growth is planktonic and the motility of microorganisms may enable taxis to certain nutrient-rich sites of the food (Wilson et al., 2002). Access of cells to nutrients and transfer of metabolites away from cells contribute to the formation of a temporarily uniform environment, until the resources are depleted, or the microbial metabolites are accumulated at self-toxic levels.

(2) If bacteria are growing in structured aqueous phase, e.g., due to addition of thickeners, or gelling (structureinducing) agents, such as gelatin, pectins, starch, gums, etc., microbial cells are immobilized within the gelled regions and constrained to grow as submerged colonies in three dimensions. Their growth rates as colonies tend to be lower than that of planktonically growing cells (Wilson et al., 2002; Theys et al., 2008; Boons et al., 2013a,b, 2014; Aspridou et al., 2014). This can be further enhanced by increasing the fat concentration on the expense of water phase, thereby increasing the size of oil droplets with concomitant trend of reversal of oil-in-water emulsion. At low fat concentrations and in the absence of any dense (3D structured) network, e.g., such as that formed by proteins, the water phase may allow cell motility that resembles planktonic growth. As the fat concentration increases and compresses water, growth is constrained and becomes colonial. In a homogeneous protein network, cocci bacterial colonies are spherical while when if fat is added within a protein network, such as cheese, cocci bacterial colonies display an irregular shape. A similar effect can be obtained by adding a structureinducing agent, such as gelatin, instead of proteins. The type and density of the gelling agent impact the growth rate of bacteria by influencing the diffusion of nutrients and metabolites to and from the colonies, respectively, as well as through the interaction of the gelling agent with inhibitory compounds, i.e., quenching or reducing diffusivity (due to entrapment) of antimicrobial agents, or due to bound of $\mathrm{NaCl}$ to the gelling agent (Boons et al., 2013b; Tack et al., 2015). The size and maximum achievable population of viable cells in colonies immobilized inside a structured matrix are affected by the proximity of colonies as well as the oxygen diffusion, with growth rate reduced in hypoxic or anoxic microenvironments (Noriega et al., 2008). The spatial distribution of cells of a population determines a critical population density level per colony, that renders the limitations in nutrient diffusion and the inhibition by metabolites sensible to adjacent colonies, leading to growth cessation (Malakar et al., 2003). Conversely, at population densities lower than the growth-limiting level, colony-tocolony interactions are negligible and cells divide without constraints. The latter critical level highly varies with the strain and the structure-inducing agent which in turn determines the chemical diffusion properties of the growth matrix.

(3) If bacteria are growing on the surface of foods, such as meat and vegetables, growth is also colonial, initially in two dimensions (mono-layer), whereas the center of colony gradually develops in the third dimension most likely upward, depending on aeration and nutrient availability. Replenishment of nutrients takes place only from the bottom or the perimeter of the colony and soon cells in the center of colony experience starvation and selftoxication. This places growth constraints to the surface colony as a whole and causes suppression of the growth rate as compared to submerged growth within the food matrix or planktonic growth. Thus, the growth rate of the aforementioned different forms of growth is known to follow the order: planktonic $\geq$ submerged $>$ surface (Wilson et al., 2002; Theys et al., 2008). It needs to be noted however, that the differences in growth rates between these three modes of growth likely range from significant (i.e., 0.5-or 1 -fold difference) to non-significant and/or not consistent, depending on the microorganism and the structure-inducing agent (Smet et al., 2015). The same accounts for lag times. In contrast, unequivocal increase in growth rate is induced by aeration of the growth medium, e.g., in shaking culture.

These observations have also been explored in relation to the stochastic behavior of individual cells growing in liquid media or immobilized inside or on the surface of solid media (Guillier et al., 2005, 2006; Manios et al., 2012; Koutsoumanis and Lianou, 2013; Tack et al., 2015). Starting from single cell level and simulating the formation of colony or the proliferation to high numbers in planktonic state, may assist in drawing useful conclusions on the expected behavior of large populations. These aspects are further discussed in the following paragraphs.

\section{Individual-based Modeling of Planktonic or Immobilized Cells}

The growth of a microbial population depends on the cumulative behavior of individual cells. As described above, a great variability is commonly evident in the growth responses, i.e., lag time, generation time and probability of growth, among individual cells of a homogeneous (or isogenic) population, due to the so-called "noise" (Locke and Elowitz, 2009). This biological variability (also termed "biovariability"; Billon et al., 1997) markedly impacts the dynamics, e.g., geometrical lag and germination time and time to reach detectable levels, of low 
populations such as $1-50$ cells and increases with the intensity of environmental stresses (Billon et al., 1997; Smelt et al., 2002, 2008; Francois et al., 2005, 2006a; Guillier et al., 2005; Guillier and Augustin, 2006; Dupont and Augustin, 2009). For instance, the distribution of germination times (lag) of individual $C$. botulinum spores became less peaky (i.e., with lower kyrtosis coefficient) and less positively skewed (i.e., skeweness factor close to 1) as the incubation temperature decreased from 37 to $20^{\circ} \mathrm{C}$, suggesting that the variance of germination times increased with the intensity of temperature stress (Billon et al., 1997; Stringer et al., 2011; Smelt et al., 2013). Variability in single cells behavior might be the result of diverse physiology of individual cells due to diverse exposure to environmental conditions in time and space of each daughter cell after the division of the mother cell. To eliminate the latter spatiotemporal diversity of the micro-environment of individual cells, microfluidic Lab-On-A-Chip systems have been proposed, such as Envirostat 2.0 (Dusny et al., 2012), which standardizes the experimental substrate under which the behavior of single cells is investigated.

However, the variable behavior of single cells is masked by the massive behavior of large populations, e.g., $>500$ cells or it is almost eliminated at optimal conditions (Llaudes et al., 2001; Smelt et al., 2002; Métris et al., 2006). Most of the available predictive models quantify the response of high microbial populations at a given set of conditions, which may be constant or varying with time. In order to model the variability of single cells (or single spores), stochastic modeling, i.e., where the input values are provided in the form of probability distributions describing the variability and uncertainty of the independent variables, may be applied, which is also the common approach in Quantitative Microbial Risk Assessment (Pérez-Rodríguez et al., 2007). Therefore, deterministic models, models based on input of single values for the independent variables, apply to population level, whereas stochastic models may describe the population dynamics taking into account the variability in both the input variables (i.e., extrinsic and intrinsic food parameters) and the responses (e.g., lag time and generation time) of microbial populations, either in large scale or at individual cell level.

Although deterministic models average the behavior of individual cells, the characteristics of the latter cannot be deduced from population measurements (Kutalik et al., 2005). Indeed, the growth of a population may be simulated by superimposing the evolution of independent subpopulations derived from single cells, each receiving a lag time value also termed "physiological lag," different from the geometrical population lag (Baranyi et al., 2009), from a specific probability distribution. The evolution of a microbial population can be modeled as a Poisson birth process with constant birth intensity parameter $\mu$ (Baranyi, 1998; Baranyi and Pin, 2001). A cell capable of dividing, will divide after an initial delay consisting of the physiological lag and the generation time of the cell. Then each cell produces a subpopulation which consists of cells growing independently in the same habitat with a constant growth rate (Baranyi, 1998; McKellar, 2001). However, deviations from this rule are likely as a result of some novel non-thermal microbial inactivation treatments, such as pulsed light and electron beam irradiation, which may diversify the growth rate, due to injury, of cell clusters within a homogeneous population exposed to the treatment (Aguirre et al., 2013, 2015). For simplification purposes, to model the process of consecutive generations of cells, it is assumed that (Baranyi, 2002; Métris et al., 2003): (i) after the first division of each cell, the daughter cells enter directly in the exponential phase, suggesting that the daughter cells have no additional lag time; (ii) daughter cells do not interact by any means, e.g., competition or quorum sensing. Both assumptions were applicable when the experimental method used to describe the variability in lag times was the time to detect visible changes in the optical density of the liquid medium containing a single cell derived by a series of 2-or 10-fold dilutions of a standard concentrated microbial suspension, or even by sorting with flow cytometry (Smelt et al., 2002, 2008; Francois et al., 2003; Standaert et al., 2005; Baranyi et al., 2009). Indeed, when the population is extremely low (i.e., down to a few cells) and the volume of the liquid surrounding medium is large, it is reasonable to speculate that the interaction between floating cells is negligible or that the probability of each cell being affected by the presence and metabolic activity of adjacent cells is very low. Individual lag times commonly follow Weibull, Gamma, Exponential, or Normal distribution (Francois et al., 2005, 2006a, 2007; Kutalik et al., 2005; Métris et al., 2006; Standaert et al., 2007). The development of sophisticated image analysis systems for realtime monitoring of single cell division (or spore germination) under the microscope, during continuous exposure of attached cells to flowing liquid media, allowed further insight in the variability assessment of single cells (Billon et al., 1997; Elfwing et al., 2004). By targeting specific cells, it was observed that the generation time of daughter cells removed after division, are not the same for all cells but they follow a distribution, the variance of which, decreases with the number of consecutive divisions (Kutalik et al., 2005; Métris et al., 2005, 2006; Pin and Baranyi, 2006).

Exposure of bacterial populations to stresses (sublethal or lethal), such as chlorine, heat shock, $\mathrm{pH}$, osmotic stresses, those related to minimal processing, such as irradiation and pulsed light, as well as sub-optimal conditions in a new environment shift the distribution of the time to first division to higher mean values (i.e., movement of mean to the right) and increase its variance (Francois et al., 2005, 2006a; Guillier et al., 2005; Guillier and Augustin, 2006; Dupont and Augustin, 2009; Aguirre et al., 2012, 2015). Furthermore, stress may decrease the probability of a single cell to initiate growth and increase the number of cells needed for growth initiation (Koutsoumanis, 2008; Dupont and Augustin, 2009). As a result, both extension of individual lag times and reduction of single cell growth probability may lead to false negative detection, due to insufficient growth above the threshold level of enrichment or no growth at all during enrichment (Dupont and Augustin, 2009). Given that stress increases the biological variability, interactions between cells within colonies (e.g., due to competition for nutrients, or the release of inhibitory metabolic products) may be an additional indigenous stress factor, which possibly increases cell lag variation, while retarding the growth of the total population (Guillier et al., 2006). 


\section{Real Time Monitoring of Single Cells and Derived Micro-colonies}

Even though the variability of growth responses (e.g., individual lag times and generation times) of planktonic cells has been extensively characterized with $\mathrm{OD}$ or microscopic measurements, the variability in relevant parameters of colonial growth (i.e., doubling of cells attached on biotic surfaces) associated with intra-colony cell-to-cell interactions are rarely quantified due to technical difficulties (Aguirre et al., 2012), nor even by direct imaging of cells when the daughter cell is removed after division. Thus, as an alternative, direct time-lapse imaging of microbial populations growing on agar surfaces of different intrinsic properties has enabled the characterization of population heterogeneity taking into account the interactions between adjacent cells (Koutsoumanis and Lianou, 2013) and colonies (Guillier et al., 2006). It may also depict the history of cells residing in different sites of a colony and their physiological adaptations, resulting from exposure to stresses, such as starvation or anoxia and affecting their subsequent resistance to inimical factors, e.g., sanitizers or lethal acid stress (Zhao et al., 2014; Tack et al., 2015). Experimental protocols for direct imaging of surface-growing cells include the gel-cassette system (Brocklehurst et al., 1997), the systems introduced by Billon et al. (1997), Niven et al. (2006), and later on adopted by Koutsoumanis and Lianou (2013), consisting of an agar layer on top of a microscope slide, covered by a cover slip, sealed with paraffin wax and placed under the microscope and the anopore strips (Ingham et al., 2005). Applications of these methods at single cell or colony level may be found for E. coli $\mathrm{O} 157: \mathrm{H} 7$, for which a comparison between growth rates estimated from viable count data and changes in colony area (in pixels) is made (Skandamis et al., 2007), Bacillus cereus in response to salinity (den Besten et al., 2007, 2010) and Salmonella showing the distribution of living and dead cells, during the evolution of colonies in time and space (Theys et al., 2009a). These techniques may also allow for identification of injured or even dead sub-populations within bacterial micro-colonies as a result of exposure to stresses or entrance into stationary phase of growth. This is achievable through the use of well-established fluorophores, such as SYTO 9, that stains living cells and propidium iodide that stains cells with impaired membranes (Bunthof et al., 2001). For instance, coupling direct cell imaging with viability staining, made possible to monitor the evolution of injury of various Lactobacillus brevis cells growing on the surface of de Man Rogosa Sharp agar, in parallel to colony growth kinetics, expressed through $\mu_{\max }$, after short exposure to peroxy-acetic acid (PAA), i.e., an strong oxidizing agent (Zhao et al., 2014). The experimental approach applied was able to unravel some interesting physiological responses that are impossible to detect by classical plate counting. In particular, not all cells that were unable to divide after exposure to PAA were appeared red (i.e., injured), suggesting that growth inhibition was not associated with membrane damage (Zhao et al., 2014). In addition, elongation or clumps of cells experiencing starvation is a very common cellular response that denotes a highly stressful physiological state of cells that cannot be detected by plate counting and probably characterize the emergence of a viable-but-not culturable sub-population (Koutsoumanis and Lianou, 2013; Zhao et al., 2014; Tack et al., 2015).

Advances in individual-based modeling (IbM) have suggested that apart from the population measurements, the complete characterization of lag time also requires the evolution of total biomass and thus, the geometrical definition of lag time is not quite reliable (Prats et al., 2008). Furthermore, since geometrical lag depends on the time required by total viable population to exceed the detection limit of the enumeration method, a part of geometrical lag does not have practically biological meaning and can also be termed "pseudo-lag" (Koutsoumanis, 2008), because growth initiation of a fast growing sub-population, which will eventually give the detection signal, might have started quite earlier.

\section{Individual-based Modeling in Foods}

The well-established variability of single cells in laboratory media is expected even more pronounced in natural food ecosystems (Ferrier et al., 2013). This may be attributable to the combination of multiple stress factors in foods, such as limitations in nutrient diffusion, competition with natural flora, accumulation of inhibitory metabolic products, structural constraints, and spatio-structural variability of microenvironments where microorganisms are located (Noriega et al., 2010). Despite the low number of studies dealing with single cell variability in foods, a common conclusion is that the behavior of low inocula (e.g., $<10 \mathrm{CFU} / \mathrm{g}$ ) cannot be accurately approximated by models based on the responses of higher inocula on the same food nor by broth-based models (Schvartzman et al., 2010; Manios et al., 2012). For instance, the time that $L$. monocytogenes required for a 100 -fold increase on vacuum packaged frankfurters stored at 4 and $8^{\circ} \mathrm{C}$, starting from 0.007 to $0.1 \mathrm{CFU} / \mathrm{g}$ was markedly higher than that expected based on the responses of 10-20 CFU/g on the safe food (Pal et al., 2009). Likewise, the simulated variability in log-numbers of L. monocytogenes cells in liver pâté at $7^{\circ} \mathrm{C}$ or lettuce and cabbage fresh cut salads, based on broth data, differed from the observed number (Francois et al., 2006b; Manios et al., 2012). Notably, Monte Carlo simulation based on stochastic description of lag times of individual L. monocytogenes cells from broth data slightly over-predicted the growth of single cells of $L$. monocytogenes after 12 days on lettuce. For instance, the model predicted that there was $60 \%$ likelihood a single cell of the pathogen to reach $1.5 \log \mathrm{CFU} / \mathrm{g}$, while the observed growth under the same probability was $1 \log \mathrm{CFU} / \mathrm{g}$ (Manios et al., 2012). In contrast, remarked under-estimation of the observed growth in cabbage was recorded, as the predictions showed that $60 \%$ of the individual cells could grow at $0.5 \log \mathrm{CFU} / \mathrm{g}$, whereas the observed growth was $2.6 \log \mathrm{CFU} / \mathrm{g}$ (Manios et al., 2012). It is imperative that the evaluation of the response of single cells in foods should receive more focus in parallel to the optimization of laboratory media assays, which provide further theoretical aspects under controlled conditions. Furthermore, improving our ability to quantitatively characterize the micro-environment surrounding single cells, e.g., by measuring the micro-scale $\mathrm{pH}$, water activity, nutrient, etc., might increase the robustness of 
predictions for growth or inactivation of these cells and explain their variable behavior based on spatio-temporal distribution of each cell (or cluster of cells) in the food matrix (Ferrier et al., 2013).

\section{CONCLUSION-FUTURE ASPECTS}

The deviation of broth-based predictions from the observed growth in foods is a well-known challenge of numerous predictive models, including some pioneer models of the last decades, addressing the issue of poor transferability of brothbased data to foods. Indeed, broth-based data are collected easily and under controlled conditions, thereby requiring low labor costs and assuring high reproducibility. However, such models do not adequately encompass the effect of critical factors explaining bacterial behavior in real foods, especially structured foods. Such critical factors may be associated with the constraints of colonial growth because of food microstructure, cell-to-cell or colony-to-colony (inter-/intra-species) interactions (Habimana et al., 2011), along with the limitations due to availability of nutrients and oxygen and/or the removal of bacterial metabolites away from colonies. The impact of these factors is amplified at low microbial populations, manifested by the stochastic behavior of single cells. Thus, extrapolating broth-based predictions of microbial growth from single cells to foods may lead to significant over- or under-estimation of actual microbial behavior in foods, with important consequences on food safety and spoilage. In this context, one of the current trends in predictive microbiology is to define food micro-architecture in quantifiable (metric) variables, so that their impact on microbial

\section{REFERENCES}

Aguirre, J., Rodríguez, M. R., González, R., and García de Fernando, G. (2013). Ebeam irradiation affects the maximum specific growth rate of Bacillus cereus. Int. J. Food Sci. Technol. 48, 382-386. doi: 10.1111/j.1365-2621.2012.03199.x

Aguirre, J. S., de Fernando, G. G., Hierro, E., Hospital, X. F., Ordóñez, J. A., and Fernández, M. (2015). Estimation of the growth kinetic parameters of Bacillus cereus spores as affected by pulsed light treatment. Int. J. Food Microbiol. 202, 20-26. doi: 10.1016/j.ijfoodmicro.2015.02.020

Aguirre, J. S., Ordóñez, J. A., and García de Fernando, G. D. (2012). A comparison of the effects of E-beam irradiation and heat treatment on the variability of Bacillus cereus inactivation and lag phase duration of surviving cells. Int. J. Food Microbiol. 153, 444-452. doi: 10.1016/j.ijfoodmicro.2011.12.012

Argyri, A. A., Panagou, E. Z., Tarantilis, P. A., Polysiou, M., and Nychas, G. J. E. (2010). Rapid qualitative and quantitative detection of beef fillets spoilage based on Fourier transform infrared spectroscopy data and artificial neural networks. Sens. Actuators B Chem. 145, 146-154. doi: 10.1016/j.snb.2009.11.052

Aspridou, Z., and Koutsoumanis, K. P. (2014). Individual cell heterogeneity as variability source in population dynamics of microbial inactivation. Food Microbiol. 45, 216-221. doi: 10.1016/j.fm.2014.04.008

Aspridou, Z., Moschakis, T., Biliaderis, C. G., and Koutsoumanis, K. P. (2014). Effect of the substrate's microstructure on the growth of Listeria monocytogenes. Food Res. Int. 64, 683-691. doi: 10.1016/j.foodres.2014.07.031

Baranyi, J., George, S. M., and Kutalik, Z. (2009). Parameter estimation for the distribution of single cell lag times. J. Theor. Biol. 259, 24-30. doi: 10.1016/j.jtbi.2009.03.023

Baranyi, J., and Pin, C. (2001). A parallel study on bacterial growth and inactivation. J. Theor. Biol. 210, 327-336. doi: 10.1006/jtbi.2001.2312 growth or inactivation is quantitatively described. Advances in relevant instrumentation, such as Nuclear Magnetic Resonance (Møller et al., 2013) for assessing the distribution of water and oil particles in emulsified foods, or the development of microelectrodes technology (Ferrier et al., 2013) measuring ion fluxes, $\mathrm{pH}$ or $\mathrm{a}_{\mathrm{w}}$ in the food microenvironment (i.e., measurements in micro-scale; Lobete et al., 2015) have enabled the collection of useful data for the above purpose. In addition, bottom-up approaches are more and more adopted by food microbiologists in the context of predictive modeling, i.e., investigating the behavior of single cells forming adjacent micro-colonies, as a means to predict the behavior of larger microbial populations in 2 or 3 dimensions, including biofilm formation (Habimana et al., 2011). Deep insights in this area, such as stochastic description of individual lag times in the form of probability distributions, are feasible via the use of time-lapse microscopy (e.g., confocal laser scanning microscope), coupled with the use of viability- respiratory activity- or gene expression-associated fluorophores at single cell level (Habimana et al., 2011; Bridier et al., 2015; Lobete et al., 2015). The latter, apart from assisting in visualization of cellular division and monitoring changes in cell number in real time, they may reveal physiological trends (e.g., virulence, stress resistance, protein expression, etc.) of single cells resulting from their interaction with the liquid or solid substrate they habituate. Finally, the challenge of integrating-omics data into predictive modeling is still open and microbiologists thrive to exploit the vast amount of such type of data collected so far so as to unravel the most critical aspects of the interaction between microorganisms and foods or between cell-to-cell interactions.

Baranyi, J., and Roberts, T. A. (1995). Mathematics of predictive food microbiology. Int. J. Food Microbiol. 26, 199-218. doi: 10.1016/01681605(94)00121-L

Baranyi, J. (1998). Comparison of stochastic and deterministic concepts of bacterial lag. J. Theor. Biol. 192, 403-408. doi: 10.1006/jtbi.1998.0673

Baranyi, J. (2002). Stochastic modelling of bacterial lag phase. Int. J. Food Microbiol. 73, 203-206. doi: 10.1016/S0168-1605(01)00650-X

Billon, C. M.-P., McKirgan, C. J., McClure, P. J., and Adair, C. (1997). The effect of temperature on the germination of single spores of Clostridium botulinum 62A. J. Appl. Microbiol. 82, 48-56. doi: 10.1111/j.1365-2672.1997.tb03296.x

Boons, K., Mertens, L., Van Derlinden, E., David, C. C., Hofkens, J., and Van Impe, J. F. (2013a). Behavior of Escherichia coli in a heterogeneous gelatindextran mixture. Appl. Environ. Microbiol. 79, 3126-3128. doi: 10.1128/AEM. 03782-12

Boons, K., Noriega, E., Van den Broeck, R., David, C. C., Hofkens, J., and Van Impe, J. F. (2014). Effect of microstructure on population growth parameters of Escherichia coli in gelatin-dextran systems. Appl. Environ. Microbiol. 80, 5330-5339. doi: 10.1128/AEM.00817-14

Boons, K., Van Derlinden, E., Mertens, L., Peeters, V., and Van Impe, J. F. (2013b). Effect of immobilization and salt concentration on the growth dynamics of Escherichia coli K12 and Salmonella typhimurium. J. Food Sci. 78, 567-574. doi: $10.1111 / 1750-3841.12067$

Booth, I. R. (2002). Stress and the single cell: intra-population diversity is a mechanism to ensure survival upon exposure to stress. Int. J. Food Microbiol. 78, 19-30. doi: 10.1016/S0168-1605(02)00239-8

Bridier, A., Hammes, F., Canette, A., Bouchez, T., and Briandet, R. (2015). Fluorescence-based tools for single-cell approaches in food microbiology. Int. J. Food Microbiol. 213, 2-16. doi: 10.1016/j.ijfoodmicro.2015.07.003 
Brocklehurst, T. F., Mitchell, G. A., and Smith, A. C. (1997). A model experimental gel surface for the growth of bacteria on foods. Food Microbiol. 14, 303-311. doi: 10.1006/fmic.1997.0098

Brul, S., Bassett, J., Cook, P., Kathariou, S., McClure, P., Jasti, P. R., et al. (2012). "Omics" technologies in quantitative microbial risk assessment. Trends Food Sci. Technol. 27, 12-24. doi: 10.1016/j.tifs.2012.04.004

Buchanan, R. L., Golden, M. H., and Phillips, J. G. (1997). Expanded models for the non-thermal inactivation of Listeria monocytogenes. J. Appl. Microbiol. 82, 567-577. doi: 10.1111/j.1365-2672.1997.tb03587.x

Bunthof, C. J., van Schalkwijk, S., Meijer, W., Abee, T., and Hugenholtz, J. (2001). Fluorescent method for monitoring cheese starter permeabilization and lysis. Appl. Environ. Microbiol. 67, 4264-4271. doi: 10.1128/AEM.67.9.42644271.2001

Cox, R. S., Dunlop, M. J., and Elowitz, M. B. (2010). A synthetic three-color scaffold for monitoring genetic regulation and noise. J. Biol. Engin. 4:10. doi: 10.1186/1754-1611-4-10

den Besten, H. M. W., Garcia, D., Moezelaar, R., Zwietering, M. H., and Abee, T. (2010). Direct-imaging-based quantification of Bacillus cereus ATCC 14579 population heterogeneity at a low incubation temperature. Appl. Environ. Microbiol. 76, 927-930. doi: 10.1128/AEM.01372-09

den Besten, H. M. W., Ingham, C. J., van Hylckama Vlieg, J. E. T., Beerthuyzen, M. M., Zwietering, M. H., and Abee, T. (2007). Quantitative analysis of population heterogeneity of the adaptive salt stress response and growth capacity of Bacillus cereus ATCC 14579. Appl. Environ. Microbiol. 73, 4797-4804. doi: 10.1128/AEM.00404-07

Dupont, C., and Augustin, J.-C. (2009). Influence of stress on single-cell lag time and growth probability for Listeria monocytogenes in Half Fraser broth. Appl. Environ. Microbiol. 75, 3069-3076. doi: 10.1128/AEM.02864-08

Dusny, C., Fritzsch, F. S. O., Frick, O., and Schmid, A. (2012). Isolated microbial single cells and resulting micropopulations grow faster in controlled environments. Appl. Environ. Microbiol. 78, 7132-7136. doi: 10.1128/AEM.01624-12

Elfwing, A., LeMarc, Y., Baranyi, J., and Ballagi, A. (2004). Observing growth and division of large numbers of individual bacteria by image analysis. Appl. Environ. Microbiol. 70, 675-678. doi: 10.1128/AEM.70.2.675-678.2004

Ferrier, R., Hezard, B., Lintz, A., Stahl, V., and Augustin, J.-C. (2013). Combining individual-based modeling and food microenvironment descriptions to predict the growth of Listeria monocytogenes on smear soft cheese. Appl. Environ. Microbiol. 79, 5870-5881. doi: 10.1128/AEM.01311-13

Francois, K., Devlieghere, F., Smet, K., Standaert, A. R., Geeraerd, A. H., Van Impe, J. F., et al. (2005). Modelling the individual cell lag phase: effect of temperature and $\mathrm{pH}$ on the individual cell lag distribution of Listeria monocytogenes. Int. J. Food Microbiol. 100, 41-53. doi: 10.1016/j.ijfoodmicro.2004.10.032

Francois, K., Devlieghere, F., Standaert, A. R., Geeraerd, A. H., Van Impe, J. F., and Debevere, J. (2003). Modelling the individual cell lag phase. Isolating single cells: protocol development. Lett. Appl. Microbiol. 37, 26-30. doi: 10.1046/j.1472-765X.2003.01340.x

Francois, K., Devlieghere, F., Standaert, A. R., Geeraerd, A. H., Van Impe, J. F., and Debevere, J. (2006a). Effect of environmental parameters (temperature, $\mathrm{pH}$ and aw) on the individual cell lag phase and generation time of Listeria monocytogenes. Int. J. Food Microbiol. 108, 326-335. doi: 10.1016/j.ijfoodmicro.2005.11.017

Francois, K., Devlieghere, F., Uyttendaele, M., Standaert, A. R., Geeraerd, A. H., Nadal, P., et al. (2006b). Single cell variability of L. monocytogenes grown on liver pâté and cooked ham at $7^{\circ} \mathrm{C}$ : comparing challenge test data to predictive simulations. J. Appl. Microbiol. 100, 800-812. doi: 10.1111/j.13652672.2006.02833.x

Francois, K., Valero, A., Geeraerd, A. H., Van Impe, J. F., Debevere, J., GarcíaGimeno, R. M., et al. (2007). Effect of preincubation temperature and $\mathrm{pH}$ on the individual cell lag phase of Listeria monocytogenes, cultured at refrigeration temperatures. Food Microbiol. 24, 32-43. doi: 10.1016/j.fm.2006.03.011

Geeraerd, A. H., Valdramidis, V. P., and Van Impe, J. F. (2005). GInaFiT, a freeware tool to assess non-log-linear microbial survivor curves. Int. J. Food Microbiol. 102, 95-105. doi: 10.1016/j.ijfoodmicro.2004.11.038

Gibson, A. M., Bratchell, N., and Roberts, T. A. (1988). Predicting microbial growth: growth responses of salmonellae in a laboratory medium as affected by $\mathrm{pH}$, sodium chloride and storage temperature. Int. J. Food Microbiol. 6, 155-178. doi: 10.1016/0168-1605(88)90051-7

Guillier, L., and Augustin, J.-C. (2006). Modelling the individual cell lag time distributions of Listeria monocytogenes as a function of the physiological state and the growth conditions. Int. J. Food Microbiol. 111, 241-251. doi: 10.1016/j.ijfoodmicro.2006.05.011

Guillier, L., Pardon, P., and Augustin, J.-C. (2005). Influence of stress on individual lag time distributions of Listeria monocytogenes. Appl. Environ. Microbiol. 71, 2940-2948. doi: 10.1128/AEM.71.6.2940-2948.2005

Guillier, L., Pardon, P., and Augustin, J.-C. (2006). Automated image analysis of bacterial colony growth as a tool to study individual lag time distributions of immobilized cells. J. Microbiol. Methods 65, 324-334. doi: 10.1016/j.mimet.2005.08.007

Habimana, O., Guillier, L., Kulakauskas, S., and Briandet, R. (2011). Spatial competition with Lactococcus lactis in mixed-species continuous-flow biofilms inhibits Listeria monocytogenes growth. Biofouling 27, 1065-1072. doi: 10.1080/08927014.2011.626124

Ingham, C. J., van den Ende, M., Pijnenburg, D., Wever, P. C., and Schneeberger, P. M. (2005). Growth and multiplexed analysis of microorganisms on a subdivided, highly porous, inorganic chip manufactured from Anopore. Appl. Environ. Microbiol. 71, 8978-8981. doi: 10.1128/AEM.71.12.8978-89 81.2005

Koutsoumanis, K. (2008). A study on the variability in the growth limits of individual cells and its effect on the behavior of microbial populations. Int. J. Food Microbiol. 128, 116-121. doi: 10.1016/j.ijfoodmicro.2008.07.013

Koutsoumanis, K. P., and Lianou, A. (2013). Stochasticity in colonial growth dynamics of individual bacterial cells. Appl. Environ. Microbiol. 79, 2294-2301. doi: 10.1128/AEM.03629-12

Kutalik, Z., Razaz, M., and Baranyi, J. (2005). Connection between stochastic and deterministic modelling of microbial growth. J. Theor. Biol. 232, 285-299. doi: 10.1016/j.jtbi.2004.08.013

Le Marc, Y., Pin, C., and Baranyi, J. (2005). Methods to determine the growth domain in a multidimensional environmental space. Int. J. Food Microbiol. 100, 3-12. doi: 10.1016/j.ijfoodmicro.2004.10.003

Llaudes, M. K., Zhao, L., Duffy, S., and Schaffner, D. W. (2001). Simulation and modelling of the effect of small inoculum size on time-to-spoilage by Bacillus stearothermophilus. Food Microbiol. 18, 395-405. doi: 10.1006/fmic.2001.0418

Lobete, M. M., Fernandez, E. N., and Van Impe, J. F. M. (2015). Recent trends in non-invasive in situ techniques to monitor bacterial colonies in solid (model) food. Front. Microbiol. 6:148. doi: 10.3389/fmicb.2015.00148

Locke, J. C. W., and Elowitz, M. B. (2009). Using movies to analyse gene circuit dynamics in single cells. Nat. Rev. Microbiol. 7, 383-392. doi: 10.1038/nrmicro2056

Malakar, K., Barker, G. C., Zwietering, M. H., and van't Riet, K. (2003). Relevance of microbial interactions to predictive microbiology. Int. J. Food Microbiol. 84 263-272. doi: 10.1016/S0168-1605(02)00424-5

Manios, S. G., Konstantinidis, N., Gounadaki, A. S., and Skandamis, P. N. (2012). Dynamics of low (1-4 cells) vs high populations of Listeria monocytogenes and Salmonella Typhimurium in fresh-cut salads and their sterile liquid or solidified extracts. Food Control 29, 318-327. doi: 10.1016/j.foodcont.2012.04.023

McDonald, K., and Sun, D.-W. (1999). Predictive food microbiology for the meat industry: a review. Int. J. Food Microbiol. 52, 1-27. doi: 10.1016/S01681605(99)00126-9

McKellar, R. C., and Knight, K. (2000). A combined discrete-continuous model describing the lag phase of Listeria monocytogenes. Int. J. Food Microbiol. 54, 171-180. doi: 10.1016/S0168-1605(99)00204-4

McKellar, R. C., Lu, X., and Delaquis, P. J. (2002). A probability model describing the interface between survival and death of Escherichia coli O157:H7 in a mayonnaise model system. Food Microbiol. 19, 235-247. doi: 10.1006/fmic. 2001.0449

McKellar, R. C. (2001). Development of a dynamic continuous-discretecontinuous model describing the lag phase of individual bacterial cells. J. Appl. Microbiol. 90, 407-413. doi: 10.1046/j.1365-2672.2001.01258.x

McMeekin, T., Bowman, J., McQuestin, O., Mellefont, L., Ross, T., and Tamplin, M. (2008). The future of predictive microbiology: strategic research, innovative applications and great expectations. Int. J. Food Microbiol. 128, 2-9. doi: 10.1016/j.ijfoodmicro.2008.06.026

McMeekin, T., Olley, J., Raktowsky, D., Corkrey, R., and Ross, T. (2013). Predictive microbiology theory and application: is it all about rates? Int. J. Food Microbiol. 29, 290-299. doi: 10.1016/j.foodcont.2012. 06.001

Mejlholm, O., Gunvig, A., Borggaard, C., Blom-Hanssen, J., Mellefont, L., Ross, T., et al. (2010). Predicting growth rates and growth boundary of Listeria monocytogenes - An international validation study with focus on processed 
and ready- to-eat meat and seafood. Int. J. Food Microbiol. 141, 137-150. doi: 10.1016/j.ijfoodmicro.2010.04.026

Métris, A., George, S. M., and Baranyi, J. (2006). Use of optical density detection times to assess the effect of acetic acid on single-cell kinetics. Appl. Environ. Microbiol. 72, 6674-6679. doi: 10.1128/AEM.00914-06

Métris, A., George, S. M., Peck, M. W., and Baranyi, J. (2003). Distribution of turbidity detection times produced by single cellgenerated bacterial populations. J. Microbiol. Methods 55, 821-827. doi: 10.1016/j.mimet.2003.08.006

Métris, A., Le Marc, Y., Elfwing, A., Ballagi, A., and Baranyi, J. (2005). Modelling the variability of lag times and the first generation times of single cells of Escherichia coli. Int. J. Food Microbiol. 100, 13-19. doi: 10.1016/j.ijfoodmicro.2004.10.004

Møller, S. M., Bertrama, H. C., Andersen, U., Lillevang, S. K., Rasmussen, A., and Hansen, T. B. (2013). Physical sample structure as predictive factor in growth modeling of Listeria innocua in a white cheese model system. Food Microbiol. 36, 90-102. doi: 10.1016/j.fm.2013.04.013

Niven, G. W., Fuks, T., Morton, J. S., Rua, S. A. C. G., and Mackey, B. M. (2006). A novel method for measuring lag times in division of individual bacterial cells using image analysis. J. Microbiol. Methods 65, 311-317. doi: 10.1016/j.mimet.2005.08.006

Noriega, E., Laca, A., and Díaz, M. (2008). Modelling of diffusion-limited growth to predict Listeria distribution in structured model foods. J. Food Eng. 87 247-256. doi: 10.1016/j.jfoodeng.2007.11.035

Noriega, E., Laca, A., and Díaz, M. (2010). Decisive role of structure in food microbial colonization and implications for predictive microbiology. J. Food Prot. 73, 938-951.

Pal, A., Labuza, T. P., and Diez-Gonzalez, F. (2009). Safety-based shelf life model for frankfurters based on time to detect Listeria monocytogenes with initial inoculum below detection limit. J. Food Prot. 72, 1878-1884.

Panagou, E. Z., Mohareb, F. R., Argyri, A. A., Bessant, C. M., and Nychas, G. J. E. (2011). A comparison of artificial neural networks and partial least squares modelling for the rapid detection of the microbial spoilage of beef fillets based on Fourier transform infrared spectral fingerprints. Food Microbiol. 28, 782-790. doi: 10.1016/j.fm.2010.05.014

Pérez-Rodríguez, F., van Asselt, E. D., Garcia-Gimeno, R. M., Zurera, G., and Zwietering, M. H. (2007). Extracting additional risk managers information from a risk assessment of Listeria monocytogenes in deli meats. J. Food Prot. $70,1137-1152$.

Pin, C., and Baranyi, J. (2006). Kinetics of single cells: observation and modelling of a stochastic process. Appl. Environ. Microbiol. 72, 2163-2169. doi: 10.1128/AEM.72.3.2163-2169.2006

Prats, C., Giró, A., Ferrer, J., López, D., and Vives-Rego, J. (2008). Analysis and IbM simulation of the stages in bacterial lag phase: basis for an updated definition. J. Theor. Biol. 252, 56-68. doi: 10.1016/j.jtbi.2008.01.019

Psomas, A. N., Nychas, G.-J., Haroutounian, S. A., and Skandamis, P. N. (2011). Development and validation of a tertiary simulation model for predicting the growth of the food microorganisms under dynamic and static temperature conditions. Comput. Electron. Agric. 76, 119-129. doi: 10.1016/j.compag.2011.01.013

Rantsiou, K., Mataragas, M., Jespersen, L., and Cocolin, L. (2011). Understanding the behavior of foodborne pathogens in the food chain: new information for risk assessment analysis. Trends Food Sci. Technol. 22, S21-S29. doi: 10.1016/j.tifs.2011.03.002

Ross, T., and Dalgaard, P. (2004). "Chapter 3: Secondary models," in Modeling Microbial Responses in Foods, eds R. C. McKellar and X. Lu (Boca Raton, FL: CRC Press), 63-150.

Schvartzman, M. S., Belessi, X., Butler, F., Skandamis, P., and Jordan, K. (2010). Comparison of growth limits of Listeria monocytogenes in milk, broth and cheese. J. Appl. Microbiol. 107, 1790-1799. doi: 10.1111/j.13652672.2010.04807.x

Skandamis, P. N., Brocklehurst, T. F., Panagou, E. Z., and Nychas, G.-J. E. (2007). Image analysis as a mean to model growth of Escherichia coli O157:H7 in gel cassettes. J. Appl. Microbiol. 103, 937-947. doi: 10.1111/j.13652672.2007.03358.x

Smelt, J. P., Stringer, S. C., and Brul, S. (2013). Behaviour of individual spores of non proteolytic Clostridium botulinum as an element in quantitative risk assessment. Food Control 29, 358-363. doi: 10.1016/j.foodcont.2012.04.021
Smelt, J. P. P. M., Bos, A. P., Kort, R., and Brul, S. (2008). Modelling the effect of sub(lethal) heat treatment of Bacillus subtilis spores on germination rate and outgrowth to exponentially growing vegetative cells. Int. J. Food Microbiol. 128, 34-40. doi: $10.1016 /$ j.ijfoodmicro.2008.08.023

Smelt, J. P. P. M., Otten, G. D., and Bos, A. P. (2002). Modelling the effect of sublethal injury on the distribution of the lag times of individual cells of Lactobacillus plantarum. Int. J. Food Microbiol. 73, 207-212. doi: 10.1016/S0168-1605(01)00651-1

Smet, C., Van Derlinden, E., Mertens, L., Noriega, E., and Van Impe, J. F. (2015). Effect of cell immobilization on the growth dynamics of Salmonella Typhimurium and Escherichia coli at suboptimal temperatures. Int. J. Food Microbiol. 208, 75-83. doi: 10.1016/j.ijfoodmicro.2015.05.011

Standaert, A. R., Francois, K., Devlieghere, F., Debevere, J., Van Impe, J. F., and Geeraerd, A. H. (2007). Modeling individual cell lag time distributions for Listeria monocytogenes. Risk Anal. 27, 241-254. doi: 10.1111/j.15396924.2006.00873.x

Standaert, A. R., Geeraerd, A. H., Bernaerts, K., Francois, K., Devlieghere, F., Debevere, J., et al. (2005). Obtaining single cells: analysis and evaluation of an experimental protocol by means of a simulation model. Int. J. Food Microbiol. 100, 55-66. doi: 10.1016/j.ijfoodmicro.2004.10.033

Stringer, S. C., Webb, M. D., and Peck, M. W. (2011). Lag time variability in individual spores of Clostridium botulinum. Food Microbiol. 28, 228-235. doi: 10.1016/j.fm.2010.03.003

Tack, I. L. M. M., Logist, F., Noriega Fernández, E., and Van Impe, J. F. M. (2015). An individual-based modeling approach to simulate the effects of cellular nutrient competition on Escherichia coli K-12 MG 1655 colony behavior and interactions in aerobic structured food systems. Food Microbiol. 45, 179-188. doi: $10.1016 /$ j.fm.2014.05.003

Tenenhaus-Aziza, F., and Ellouze, M. (2014). Software for predictive microbiology and risk assessment: a description and comparison of tools presented at the ICPMF8 Software Fair. Food Microbiol. 45, 290-299. doi: 10.1016/j.fm.2014.06.026

Theys, T. E., Geeraerd, A. H., Devlieghere, F., and Van Impe, J. F. (2009a). Extracting information on the evolution of living- and dead-cell fractions of Salmonella Typhimurium colonies in gelatine gels based on microscopic images and plate-count data. Lett. Appl. Microbiol. 49, 39-45. doi: 10.1111/j.1472765X.2009.02623.x

Theys, T. E., Geeraerd, A. H., and Van Impe, J. F. (2009b). Evaluation of a mathematical model structure describing the effect of (gel) structure on the growth of Listeria innocua, Lactococcus lactis and Salmonella Typhimurium. J. Appl. Microbiol. 107, 775-784. doi: 10.1111/j.1365-2672.2009.04256.x

Theys, T. E., Geeraerd, A. H., Verhulst, A., Poot, K., Van Bree, I., Devlieghere, F., et al. (2008). Effect of $\mathrm{pH}$, water activity and gel micro-structure, including oxygen profiles and rheological characterization, on the growth kinetics of Salmonella Typhimurium. Int. J. Food Microbiol. 128, 67-77. doi: 10.1016/j.ijfoodmicro.2008.06.031

Whiting, R. C., and Buchanan, R. L. (1993). A classification of models for predictive microbiology. Food Microbiol. 10, 175-177.

Whiting, R. C. (1993). Modeling bacterial survival in unfavorable environments. J. Ind. Microbiol. 12, 240-246. doi: 10.1007/BF01584196

Wilson, P. D. G., Brocklehurst, T. F., Arino, S., Thuault, D., Jakobsen, M., Lange, M., et al. (2002). Modelling microbial growth in structured foods: towards a unified approach. Int. J. Food Microbiol. 73, 275-289. doi: 10.1016/S01681605(01)00660-2

Zhao, Y., Knøchel, S., and Siegumfeldt, H. (2014). In situ examination of Lactobacillus brevis after exposure to an oxidizing disinfectant. Front. Microbiol. 5:623. doi: 10.3389/fmicb.2014.00623

Conflict of Interest Statement: The authors declare that the research was conducted in the absence of any commercial or financial relationships that could be construed as a potential conflict of interest.

Copyright $\odot 2015$ Skandamis and Jeanson. This is an open-access article distributed under the terms of the Creative Commons Attribution License (CC BY). The use, distribution or reproduction in other forums is permitted, provided the original author(s) or licensor are credited and that the original publication in this journal is cited, in accordance with accepted academic practice. No use, distribution or reproduction is permitted which does not comply with these terms. 


\title{
Recent trends in non-invasive in situ techniques to monitor bacterial colonies in solid (model) food
}

\author{
María M. Lobete ${ }^{1,2}$, Estefania Noriega Fernandez ${ }^{1,2}$ and Jan F. M. Van Impe ${ }^{1,2}$ * \\ 1 Flemish Cluster Predictive Microbiology in Foods, Leuven, Belgium \\ ${ }^{2}$ Chemical and Biochemical Process Technology and Control, Department of Chemical Engineering, Katholieke Universiteit Leuven, Leuven, Belgium
}

\section{Edited by:}

Sophie Jeanson, Institut National de la Recherche Agronomique, France

Reviewed by:

Raivo Vilu, Tallinn University of Technology, Estonia

Jean-Christophe Augustin, Ecole

Nationale Vétérinaire d'Alfort, France

*Correspondence:

Jan F. M. Van Impe, Chemical and Biochemical Process Technology

and Control, Department of

Chemical Engineering, Katholieke

Universiteit Leuven, Willem de

Croylaan 46, B-3001 Leuven,

Belgium

e-mail: jan.vanimpe@cit.

kuleuven.be
Planktonic cells typically found in liquid systems, are routinely used for building predictive models or assessing the efficacy of food preserving technologies. However, freely suspended cells often show different susceptibility to environmental hurdles than colony cells in solid matrices. Limited oxygen, water and nutrient availability, metabolite accumulation and physical constraints due to cell immobilization in the matrix, are main factors affecting cell growth. Moreover, intra- and inter-colony interactions, as a consequence of the initial microbial load in solid systems, may affect microbial physiology. Predictive food microbiology approaches are moving toward a more realistic resemblance to food products, performing studies in structured solid systems instead of liquids. Since structured systems promote microbial cells to become immobilized and grow as colonies, it is essential to study the colony behavior, not only for food safety assurance systems, but also for understanding cell physiology and optimizing food production processes in solid matrices. Traditionally, microbial dynamics in solid systems have been assessed with a macroscopic approach by applying invasive analytical techniques; for instance, viable plate counting, which yield information about overall population. In the last years, this approach is being substituted by more mechanistically inspired ones at mesoscopic (colony) and microscopic (cell) levels. Therefore, non-invasive and in situ monitoring is mandatory for a deeper insight into bacterial colony dynamics. Several methodologies that enable highthroughput data collection have been developed, such as microscopy-based techniques coupled with image analysis and OD-based measurements in microplate readers. This research paper provides an overview of non-invasive in situ techniques to monitor bacterial colonies in solid (model) food and emphasizes their advantages and inconveniences in terms of accuracy, performance and output information.

Keywords: bacterial colonies, growth dynamics, solid food (model) system, image analysis, food structure, optical density, microcalorimetry

\section{EFFECT OF SOLID STRUCTURES ON THE GROWTH OF BACTERIAL POPULATIONS}

Microbial behavior is determined by structure as much as by environmental conditions and the chemical composition of the media (Robins and Wilson, 1994; Wilson et al., 2002; Antwi et al., 2006, 2007; Theys et al., 2008). In solid structures, substrates diffuse into the colonies and metabolic products outside, triggering concentration gradients in and around the colony, that concern cells availability to oxygen and nutrients (Wimpenny and Coombs, 1983; Wimpenny et al., 1995; Walker et al., 1997; Malakar et al., 2000, 2003; Kabanova et al., 2013). The outwards diffusion of metabolic acids in bacterial colonies gives rise to $\mathrm{pH}$ gradients, causing a potential degree of biochemical heterogeneity within and around the colony cells (Walker et al., 1997; Malakar et al., 2000). Studies focused on oxygen penetration's depth and its diffusion through the matrix have revealed to play also a key role on bacterial colonies growth, regarding their surface or submerged character (Wimpenny and Coombs, 1983; Peters et al., 1987;
Robinson et al., 1991). Solid structures has also been reported to affect certain bacterial characteristics, e.g., cell development, morphology, membrane permeability, surface tension, osmotic pressure and metabolism, as well as cell viability and physiological state (Dervakos and Webb, 1991; Wilson et al., 2002; Meldrum et al., 2003). Some authors stated that cell immobilization in a solid environment can also generate changes in cell susceptibility to antimicrobial compounds (Walker et al., 1997; Malakar et al., 2000; Skandamis et al., 2000; Dens and Van Impe, 2001) as well as acid tolerance responses (Malakar et al., 2000; Antwi et al., 2007).

Therefore, it seems of outmost importance to study colony behavior in solid structures, since most of the studies in literature have focused on planktonic growth in liquid systems. In fact, trends in predictive microbiology are moving toward a more realistic approach of microbial behavior in structured food products, including the study of surface and submerged colonies. This work gives an overview of the different available 
techniques to characterize colony growth in solid model food systems.

\section{STUDYING BACTERIAL COLONIES IN SOLID MODEL FOOD SYSTEMS \\ INVASIVE TECHNIOUES}

To date, several approaches have been developed for a better understanding of bacterial colony behavior in solid systems. Most traditional measuring techniques provide information of overall microbial populations through invasive procedures, like viable plate count and microelectrodes. Traditional techniques enable growth dynamics estimation, the measurement of environmental conditions, etc. Hereunder are compiled some representative achievements in this field.

To study colony behavior at laboratory scale, bacterial colonies are often obtained from the inoculation of different solid culture media prepared by adding different gelling agents. Agar and gelatine are widely used in food microbiology, although their relevance is restricted to products with gelled microstructure like frankfurters (Mertens et al., 2009). In order to cover a wider range of model food systems, some authors assayed with different hardening agents such as xanthan gum, Carbopol, $\kappa$-carrageenan (Mertens et al., 2009, 2011; Noriega et al., 2010; Boons et al., 2013a) and Maxiren 180 (DSM Food Specialities, France), a coagulant agent used by Jeanson et al. (2011) for a model cheese. Studies on real solid food have also been performed, e.g., Katsaras and Leistner (1988) described for the first time the growth of submerged bacteria in fermented sausages; Noriega et al. (2010) described the role of the food structure in bacterial colonization of poultry products. The study of bacterial colonies also requires an adequate experimental setup to hold the solid structure in which colonies will be cultured, e.g., plastic or glass petri dishes and conical tubes. Special attention draws the gel-cassette system designed by Brocklehurst et al. (1995) at the Institute of Food Research (IFR, Norwich, UK). This system consists of a frame sealed with gas-permeable plastic film that holds the inoculated gelled media between two PVC transparent windows. The gelled media needs a separate preparation in order to be inoculated and introduced in the system before solidifying. Once the cassette is filled, it is sealed with the plastic films, being then ready for incubation. Sampling for viable count experiments from the gel-cassette requires blending the media to take an aliquot; while for colony observation it can be directly couple to a microscopy device. The gel-cassette is widely used to develop either submerged or surface colonies and has helped assessing overall population dynamics of several pathogenic bacteria, e.g., Salmonella typhimurium (Brocklehurst et al., 1995), Listeria monocytogenes (Meldrum et al., 2003) and mixed bacterial co-cultures (Tsigarida et al., 2003).

Among the traditional techniques to determine bacterial growth in solid structures, viable plate count remains as the most commonly used procedure. This technique generally entails the sampling of an aliquot from the inoculated system under study, its homogenization and dilution. Finally, the sample is spread on the corresponding agar plates for the following viable counting. This technique has been used to determine the overall growth dynamics of bacterial populations and thus, to elucidate the potential effect of different environmental factors. Brocklehurst et al. (1995) assessed the effect of transient temperatures on the growth dynamics of S. Typhimurium. Noriega et al. (2013) used global viable counts to determine the effect of medium structure on the sublethal injure of S. Typhimurium, L. monocytogenes and Escherichia coli colonies. In Skandamis et al. (2007) viable plate count was compared with a non-invasive technique to study the effect of temperature and $\mathrm{pH}$ on the growth of $E$. coli colonies cultured in the gel-cassette. Although this methodology is widely applied in the study of bacterial colonies, viable plate count gives information of overall population dynamics, but not from the individual colonies; moreover, it requires a tedious, time consuming and costly work (Guillier et al., 2006; Jeanson et al., 2011; Mertens et al., 2012; Koutsoumanis and Lianou, 2013). Additionally, the heterogeneous behavior among individual cells is driving researches to study cell colonies individually, instead of as a whole population.

As previously described, colony growth in solid systems is affected by several diffusion limitations responsible for gradients of $\mathrm{pH}$, oxygen, metabolites, etc. For a deeper insight in these localized gradients, an invasive technique based on direct measurements with microelectrodes has been developed. Wimpenny and Coombs (1983) published the first work in which microelectrodes were applied to measure oxygen penetration. Colonies of Bacillus cereus were cultured on the surface of tryptone soya broth agar (TSBA) contained in petri dishes and oxygen measurements were performed with an oxygen-sensitive microelectrode mounted on a prior micromanipulator. On the other hand, it has been reported the potential inaccuracy in the use of microelectrodes, due to oxygen leakage around the electrode and poisoning tip (Tammam et al., 2001). Microelectrodes have also been applied for measuring $\mathrm{pH}$ gradients, e.g., Walker et al. (1997) described the $\mathrm{pH}$ gradients within and around surface colonies of S. Typhimurium grown in an adapted gelatin cassette system. This technique is usually combined with viable plate count, so that, information about colony growth assist in interpreting the obtained measurements. Nowadays, few tools are available to monitor and identify the metabolic release patterns of growing microbial colonies. Among them, matrixassisted laser desorption/ionization-time of light (MALDI-TOF) imaging mass spectrometry (IMS) has recently been proposed as a valuable source to detect and to (2D) and (3D) visualize the distribution of metabolites produced by microbial colonies (Gonzalez et al., 2012; Fang and Dorrestein, 2014). Through IMS, the whole bacterial colony is examined including the surrounding agar medium, defining a raster composed of greater that 1000 laser points of data collection, increasing thus the likelihood of detecting unique, discrete ion distribution patterns and hidden molecular phenotypes (Gonzalez et al., 2012). In addition, its combination with MALDI-TOF imaging unravels to visualize the spatial distribution of the detected compounds.

The study of microbial colonies and their peculiarities has also been extended to microbial identification and characterization. It is worth to mention certain techniques that although invasive, approach microbial colonies from an individual point of view. For instance, Fourier transform infra-red (FT-IR) spectrometry provides fingerprint-like patterns of microorganisms that enable to identify different species and strains due to their specific 
spectra. The efficiency of this method has been demonstrated for several bacterial species such as Staphylococcus, Streptococcus, Clostridium and Legionella (Helm et al., 1991), among others. Thi et al. (2003) assessed an improvement of this technique by coupling the FT-IR spectrometer with a light microscope and obtain microscopic spectra that provide information on the number, size and shape of the micro-colony cells. Furthermore, this technique was extended in Thi and Naumann (2007) to study the spatial heterogeneity of cell growth within macro-colonies. Cells were grown in agar under optimal conditions in order to get macrocolonies with a diameter of about $3-5 \mathrm{~mm}$. These macro-colonies were excised from the agar, frozen at $-70^{\circ} \mathrm{C}$ and cryosectioned in slices of $20 \mu \mathrm{m}$ for further FT-IR mapping measurements. Obtained spectra revealed a substantial spatial heterogeneity of cell composition among the microorganisms studied (Legionella bozemanii, Bacillus megateriuma and Candida albicans).

Invasive techniques have clearly contributed to increase the knowledge about bacterial colonies behavior; however, their invasive character, the subsequent sample discard and the lack of individual information gathered at the colony level are disadvantages to be overcome. In order to collect more accurate information and fundamental knowledge, non-invasive analytical techniques focused on mesoscopic and microscopic assessments of colony growth, are replacing the traditional ones (Mertens et al., 2012).

\section{NON-INVASIVE AND IN SITU TECHNIQUES}

As previously described, most studies involving bacterial colonies are based on colony estimation of overall cell population dynamics in solid systems; however, an individual approach is required for a deeper insight in single colony behavior. Latest trends are moving toward the development of non-invasive techniques that enable to monitor bacterial colonies without altering their environment or subsequent sample discard. These techniques offer the advantage of obtaining quantitative data, faster and more accurately. Hereunder some of these novel techniques are grouped by the applied principle, which to date are mainly imaging techniques, optical density (OD) and microcalorimetric techniques. Nevertheless, the transition from traditional to novel techniques is gradually occurring since the latter also present some limitations. Under this situation, it can be observed that in many of the works here reviewed, novel techniques are either combined or compared with traditional ones, such as viable plate count.

\section{Imaging techniques}

The use of microscopy-based methods enables to monitor growth of bacterial colonies in real time and to estimate different parameters related to colony evolution. This can be obtained through a further analysis of the obtained images. These methods are gaining interest in the field of food microbiology, since image analysis programs and automation software tools make data processing more feasible (Skandamis et al., 2007). Hereunder are some of the most applied techniques in this field detailed.

Light microscopy and digital camera coupling. Direct colony observation through a light microscope provides a useful tool to monitor certain colony parameters such as the colony radius and their area evolution on the surface. The combination of a light microscope with a digital camera offers the possibility to immortalize the visualized image for further process and study. Several studies based on these techniques have been conducted to estimate colony dynamics, i.e., growth rate and lag phase, which are generally the most representative parameters to be identified (Guillier et al., 2006; Rasch et al., 2007; Skandamis et al., 2007; Koutsoumanis and Lianou, 2013). With this aim, Guillier et al. (2006) introduced a new method to estimate and compare individual lag times of either stressed or exponentially growing colonies of L. monocytogenes. Obtained results were afterwards compared with the individual lag time distributions observed in broth. In this work, the setup for surface colony development was agar plates, on which the colonies were monitored with a digital camera PixelFly (Photon Lines) and an automatic image processing system. Initially, suspensions of L. monocytogenes cells were disposed with a spiral plater on plates of tryptone soya agar enriched with yeast extract (TSAYE). During incubation, images were taken at constant time intervals of $10 \mathrm{~min}$. The imaging set-up for this study consisted of a circular motorized platform OptoSigma (Photon Lines, Marly-le-Roy, France), that was coupled with the digital camera and enabled to study four plates simultaneously. While the colonies were growing, the intensity of the pixels in the obtained images was also increasing and this increment was then related to the lag phase. In this context, the time required for a colony to reach a surface threshold is considered proportional to the lag time of the cell initiating the colony, as also assumed for turbidity-based methods. This work presents a valuable method for studying colony growth dynamics since results showed means and standard deviation values similar to those obtained in broth, as well as no clear difference between liquid and solid conditions.

One of the main systems used for obtaining and visualizing submerged and surface colonies, is the gel-cassette. The gel cassette set-up was already widely used to estimate population growth dynamics in solid systems and it was at the beginning of the 21st century when its specific configuration with two transparent windows, inspired the coupling with microscopy techniques, enabling thus in vivo colony visualization. Skandamis et al. (2007) applied this combination of devices, a light microscope coupled with a digital camera and the gel-cassette to determine the effect of temperature and $\mathrm{pH}$ on the growth rate of $E$. coli O157:H7 colonies. The gel-cassette system was aseptically filled in with brain heart infusion agar (BHIA) adjusted at different $\mathrm{pH}$ values and then inoculated. Afterwards, it was coupled to a microscope with a high-sensitivity SSC-DC50AP digital camera (Sony Corp, Tokyo, Japan) that allowed to capture images of the growing colonies. The evolution of the colony surface was monitored with $40 \times$ objective lens from a light microscope and expressed in $\log _{10}$ transformed pixels. Surface area data were used to estimate maximum growth rates $\left(\mu_{\max }\right)_{\mathrm{A}}$ by applying the model of Baranyi and Roberts (1994). The observed $\left(\mu_{\max }\right)_{\mathrm{A}}$ values were fitted to a quadratic secondary model to express the natural logarithm of the $\left(\mu_{\max }\right)_{\mathrm{A}}$ as a function of temperature and $\mathrm{pH}$. Based on a correlation between the observed $\left(\mu_{\max }\right)_{\mathrm{A}}$ and the observed viable count-based $\mu_{\max }\left(\mu_{\max }\right)_{\mathrm{VC}}$, the predicted $\left(\mu_{\max }\right)_{\mathrm{A}}$ values were transformed into predicted $\left(\mu_{\max }\right)_{\mathrm{VC}}$. This model was validated by graphical comparison of the predicted 
$\left(\mu_{\max }\right)_{\mathrm{VC}}$ with published $\left(\mu_{\max }\right)_{\mathrm{VC}}$ values from E. coli O157:H7 in broth and different foods, as well as by mathematical means, i.e., the bias and accuracy performance indices (Ross, 1996). These data were gathered from ComBase database. Results showed that the model performed well with published responses of E. coli O157:H7 in broth, although a poorer agreement of the model was obtained from the correspondent responses of $E$. coli $\mathrm{O} 157: \mathrm{H} 7 \mathrm{in}$ food. It can be concluded that the use of $\left(\mu_{\max }\right)_{\mathrm{A}}$ is an adequate alternative in the development of predictive models to describe the $\left(\mu_{\max }\right)_{\mathrm{VC}}$ and a potential alternative to traditional viable plate count.

A further step in surface colony image monitoring with a light microscope was presented by Koutsoumanis and Lianou (2013). This research evaluated image series obtained at short time intervals, sequentially compiled and transformed into a video. The aim of this study was to assess the heterogeneity in the growth dynamics of micro-colonies emerging from single cells of S. Typhimurium investigation. Bacterial colonies from individual cells were grown on TSA disposed on a glass slide, which was covered with a coverslip. This set-up allowed to directly observe under the z-motorized microscope (Olympus BX61; Olympus, Tokyo, Japan), equipped with a high resolution device camera. Images were obtained with phase-contrast time-lapse microscopy and compiled to create the video. This video was a useful tool to characterize the cells in terms of division times, cell length and growth kinetics. Results showed a high heterogeneity in the growth behavior if individual cells are compared. This heterogeneity became higher for those cells that gave rise to small colonies. Generally, the experimental error from the application of monitoring methods, remains as the main constrain at describing colonial behavior. Time-lapsed microscopy method described in this paper, eliminated this limitation since it allowed to count each cell with time in each micro-colony.

Fluorescence and confocal laser scanning microscopy. Fluorescence microscopy technique is based on detecting the emitted fluorescence by fluorescent molecules. The excitation of these molecules is followed by an emission of a band of light with a specific wavelength, being the excitation-emission spectra specific for each molecule. The use of fluorophores allows to stain target components such as, proteins, oil droplets, carbohydrates, etc., and thus, to visualize them. Several works have been presented to study bacterial colonies by applying this principle.

Malakar et al. (2000) presented one of the first works involving gel-cassette with a fluorescence microscope to monitor bacterial colonies and experimentally confirming the presence of $\mathrm{pH}$ microgradients within and around them. In this study, colonies of Lactobacillus curvatus were grown in De Man, Rogosa, Sharpe (MRS) broth with bovine gelatine as gelling agent and supplemented with Oregon Green ${ }^{\mathrm{TM}}$, a fluorescent dye which intensity was linearly related to a $\mathrm{pH}$ range; once prepared the mixture, it was aseptically introduced in the gel-cassette system for further analysis. Subsequently, a fluorescence microscope (Nikon, Surrey, UK) with the following filters, excitation $(440 \pm 10 \mathrm{~nm}$, $510 \pm 11.5 \mathrm{~nm})$, emission $(535 \pm 11.5 \mathrm{~nm})$ and a dichroic mirror (540 nm, long pass) was set up with the gel-cassette system and serial measurements of fluorescence intensity in the inoculated media were taken. These images were obtained after focusing on a defined plane between the bottom and top of each cassettewindow and exciting this area with defined light and wavelengths. Furthermore, light transmission images were taken in order to determine the colony radius, once they were visible and put into focus. By representing the measured radius and $\mathrm{pH}$ values, $2 \mathrm{D}$ $\mathrm{pH}$ maps were elaborated. Finally, the superimposition of the $\mathrm{pH}$ maps with the transmission images, helped to reveal the $\mathrm{pH}$ profiles in and around the colony. As the authors stated, this technique was the first approach that allowed to monitor colony growth non-invasively, as well as to reinforce the concept of $\mathrm{pH}$ gradients in solid systems, being more representative of what happens in a colony in real time.

CLSM works on the same principle as fluorescence microscopy although it presents certain advantages, e.g., better resolution images due to the pinhole that eliminates out-of-focus light and the possibility to observe thicker samples, making optical sections at different depths that enables to reconstruct a 3D-image. This device, coupled with the gel-cassette was applied by Jeanson et al. (2011) to study colony size and distribution as a function of the inoculum level in a model cheese. In literature, has often been object of study the effect of the initial microbial load on colony size and distribution. High initial inoculum levels promote small colonies closely located; in contrast, low initial cell densities lead to large colonies separated by long distances (Malakar et al., 2002, 2003; Theys et al., 2009). As a consequence, inter- and intracolony interactions appear, affecting bacterial behavior. Intercolony interactions are typically found between small colonies separated by short distances in which the fast diffusive transport has been described as comparable phenomena to convection in broth (Malakar et al., 2002, 2003). In contrast, intra-colony interactions are dominant in large colonies formed from a low inoculation levels. In this scenario, diffusion becomes a constraint since it is not able to cope with the demands of the cells situated in the center of the colony; as a consequence, this delayed molecular transport within the colony triggers the interaction between the inner cells (Malakar et al., 2002, 2003). In this context, the work of Jeanson et al. (2011) aimed at describing this phenomenon in a cheese matrix with immobilized bacteria whose metabolism is the main responsible for its ripening. The model cheese matrix was developed at laboratory level with the retentate from milk ultrafiltration (UF) and coagulant agent Maxiren 180 (DSM Food Specialities, France). This model system was afterwards introduced in a gel cassette and inoculated with different levels of Lactococcus lactis, in order to observe the bacterial distribution under the CLSM. The selected strain produces a green fluorescent protein (GFP) that can be linked to the metabolically active state of the cells and therefore, observed under the microscope. Several CLSM photographs of the fluorescent colonies were taken aiming at characterizing their spatial distribution in the model cheese. This distribution resulted to be random in the whole structure, independently of the inoculum level. Nevertheless, the initial density was found to determine the spatial separation between the colonies, as previously reported by Malakar et al. (2003). Traditional viable plate counting was also carried out, confirming that independently of the initial inoculation level, the final stationary population remains equal. Besides colony 
size and distribution, Floury et al. (2013) used CLSM coupled with the gel-cassette system to study the potential porosity of the L. lactis colonies to certain compounds in a cheese model system. As above described, cheese ripening depends on the metabolism of immobilized bacteria. Thus, cheese ripening can be affected by diffusional limitations of nutrients and metabolites within and around the colonies. For this case study, colony porosity to different sizes of dextran molecules (from 4.4 to $155 \mathrm{kDa}$ ) was assessed in the model cheese system and in M17-agar. Both media were aseptically prepared, inoculated and disposed in mini gel-cassette systems for directly observation under CLSM. For the porosity observation, the chosen dextrans were fluorescently labeled with Rhodamine B isothiocyanate (Sigma Aldrich, SaintQuentin Falavier, France) and viable and total bacterial counts were performed with the LIVE/DEAD ${ }^{\circledR}$ Bacterial Viability Kit (BacLigh $^{\mathrm{TM}}$, Molecular Probes, Invitrogen). Obtained images were also used to study the different morphologies of the colonies depending on the media; while in the model cheese they were spherical, colonies grown in M17-agar showed under the same growing conditions, a lenticular shape.

Besides the gel-cassette, other setups like chambered cover glass are applied for CLSM studies. Jeanson et al. (2013) used CLMS with CoverWell imaging chambers (Sigma-Aldrich, Quentin Fallavier, France) to assess the $\mathrm{pH}$ around colonies of L. lactis inoculated in the cheese model system with different inoculum levels. Bacterial colonies were visualized with the fluorescent dye SYTO-9 (Molecular Probes, Invitrogen, Villebonsur-Yvette, France), which was excited at $488 \mathrm{~nm}$ and detected at $515 \pm 15 \mathrm{~nm}$. This visualization confirmed that the low inoculum levels gave rise to bigger colonies. A pH sensitive probe C-SNARF4F (Molecular Probes, Invitrogen, Villebon-sur-Yvette, France) was used to monitor micro-pH around the colonies. This CSNARF-4F was excited at $543 \mathrm{~nm}$ and detected at $590 \pm 15 \mathrm{~nm}$ and over $650 \mathrm{~nm}$, enabling afterwards to calculate the ratio $(R)$ from these two peaks. Measurements were taken along $100 \mu \mathrm{m}$ from the edge of the colony at $10 \mu \mathrm{m}$ steps from opposite sides of the colony. In order to correlate $R$ values with $\mathrm{pH}$, macro-pH measurements were performed with a $\mathrm{pH}$ meter (Inlab $\mathrm{pH}$ level 1; WTW, Germany) and pH electrode (Sebtix 41; WTW, Germany) in flasks containing the inoculated model cheese and incubated under the same conditions as the imaging chambers. Results showed that lower inoculum levels yielded to lower acidification kinetics; however, from the micro-pH measurements around the colonies, no microgradients were identified independently of the size of the studied colonies. The absence of these microgradients reveals, for the first time, that the $\mathrm{pH}$ perceived by the cells in a model cheese while acidification, is actually the macropH. Boons et al. (2013b) also used CLSM with imaging well chambers (Nunc Lab-Tek (USA) chambered borosilicate coverglass system) to determine the effect of the media composition and microstructure. This paper describes for the first time the behavior of $E$. coli in a heterogeneous protein-polysaccharide solid media with CLSM. The main novelty in this work was to use a gelatine-dextran mixture at different concentration ratios, which led to different microstructures characterized by a phaseseparation, affecting thus bacterial behavior. This phenomenon was observed in the system thanks to the Rhodamine B, which enabled gelatine phase visualization. Initially, increasing gelatine concentrations promoted the transition from a homogeneous to a heterogeneous structure in which dextran spheres appeared in the gelatine matrix. However, if the gelatine phase concentration was also increased, a phase inversion occurred and gelatine spheres appeared in the dextran matrix. This phase inversion induced E. coli colonies to grow as diffuse strings instead as defined spheres. This morphological characterization was observed thanks to in the Venus fluorescent protein included in the E. coli strain, which expression enabled to visualize the bacterial cells with CLMS. In spite of these morphological changes, colony development occurred exclusively in the dextran phase. The same imaging technique was applied in Boons et al. (2014), although viable plate counting was also performed to estimate the microbial dynamics of $E$. coli in the heterogeneous microstructure, in order to decipher relation between micro- and macroscale results.

Imaging techniques for identification. Besides monitoring microbial growth, imaging techniques are also a useful tool to study specific microbial colony characteristic, such as strain identification. In this context, label-free bacterial colony phenotyping technology, also called bacterial rapid detection using optical scattering technology (BARDOT), has shown promising results in bacterial identification and classification. Kim et al. (2014) presented a theoretical model in order to explain the underlying mechanism of speckle formation by the colonies from Bacillus. Laser-based optical sensor interrogated the entire volume of the colonies in order to gather information that could be encoded in the far-field scatter patterns. This technique was also assessed by Bae et al. (2010) on bacterial colonies of S. Montevideo, L. monocytogenes and E. coli, obtaining results that correlate colony morphology and growing characteristics with characteristic diffraction patterns.

It can be concluded that a wide range of applications are available for studying bacterial colonies with non-invasive imaging methods, although many authors still include traditional invasive techniques in their studies, like viable plate count, to enumerate total cell population. Experimental setups based on microscopy devices coupled with high resolution cameras and, e.g., the gelcassette system, provide a valuable and promising tool to monitor individual cell colonies. However, their routine application in food microbiology remains a challenge since they also have some limitations.

For instance, the use of the gel-cassette limits the number of experimental conditions that can be assessed simultaneously (Mertens et al., 2012), as well as the tedious required work for the data analysis. These limitations point out the need for further studies to optimize available non-invasive methods to monitor colony behavior.

\section{Alternative methods}

Optical density measurements have been traditionally applied as an indirect method to monitor individual cell growth, which is related to the media absorbance evolution. Based on Beer's law, OD is proportional to microbial concentration, as described by Koch (1981). In diluted suspensions, most bacteria have nearly 
the same absorbance per unit of dry weight concentration, independently of cell size. This method appeared as an alternative to more laborious and time consuming techniques such as viable plate count. OD-based methods represent a useful tool for growth parameter estimation due to its non-destructive character and its ability to fast provide high-throughput data; furthermore, it is not expensive and relatively easy to automate in comparison to other classical methods (Mertens et al., 2012). Several authors have applied this method to address microbial growth of freely suspended cells (McClure et al., 1993; Dalgaard et al., 1994; Nerbrink et al., 1999; Dalgaard and Koutsoumanis, 2000; Métris et al., 2006). Traditionally, measurements were taken one by one in spectrophotometers, requiring hard work and limited number of samples; however, this technique has evolved and more advanced devices, such as the Bioscreen $C^{\circledR}$, Versamax ${ }^{\circledR}$, etc., have appeared in the market. These latest set-ups are provided with reading chambers for microtiter plates that contain numerous wells; moreover, temperature can be programmed as well as the number of measurements and the interval time. These characteristics enable to simultaneously study different environmental conditions due to the multiple and independent wells per plate and a high-throughput non-invasively data collection. For a direct data processing, several software packages have been developed that can be coupled with the mentioned experimental devices.

OD-based techniques have been recently applied for colony growth characterization. Mertens et al. (2011) used the SpectraMax $\mathrm{M} 2^{\mathrm{e}}$ microplate reader to assess the effect of a solid environment on the growth/no growth (G/NG) boundaries of Zygosaccharomyces bailii, in comparison to liquid media. This work supposed the first approach of an OD method to study microbial colonies. In this work, the structured model system was based on Carbopol and xanthan gum as gelling agents, which were independently tested and, in the case of Carbopol, prepared at different concentration ratios. Rheological measurements were applied to characterize the different media based on the Carbopol concentration. From this measurements it was shown that higher concentration levels gave rise to highly structured systems and those with intermediate levels of Carbopol induced low structured systems. After preparation, the different media were inoculated and distributed in the 48-well microtiter plates. Prior to inoculation, a preculture of $Z$. bailii was obtained under optimal growing conditions and serially diluted to reach an inoculation level of $5 \times 10^{4} \mathrm{CFU} / \mathrm{mL}$. Due to the planktonic character of the cells in the preculture, the last dilution before inoculation was carried out in the same structured model system used afterwards. This specific model system, developed by Mertens et al. (2009), aims at obtaining similar conditions to those found in acidic sauces like ketchup, which is commonly spoiled by $Z$. bailii. Including these compounds as gelling agents supposed a novelty in the area, as well as the use of $Z$. bailii in solids, since the majority of studies to date have focused on agar or gelatine and on pathogenic bacteria, respectively. Once the microtiter plates were filled in with the inoculated media and the equipment conditions settled, measurements were programmed to be taken at regular time intervals. For the experiments with planktonic cells, OD was determined at one single point of each well in the microtiter plate; however, in the case of the solid structure, the scan method was performed by measuring every well at nine different positions. Afterwards, obtained values were used to plot OD curves. Results showed that yeast colonies development depended on the structured degree of the media. In one hand, intermediately structured Carbopol media led to numerous and homogeneously distributed colonies, easily identified with a shift in the OD-based growth curves. On the other hand, highly structured Carbopol media limited colony formation to certain environmental conditions. For environmental conditions close to the G/NG boundaries, between none and small few colonies appeared and remained mainly undetectable. This supposes a limitation of the method since just one single cell can yield a colony, risking food safety and quality. Authors concluded that it would be more realistic to independently consider the nine measurements taken at different positions of each well, instead of as mean values, for more accurate identification and definition of colonial behavior. This method was improved in Mertens et al. (2012) to study the colony growth of $E$. coli at optimal temperature $\left(30^{\circ} \mathrm{C}\right)$. As in the previous study, the area scan method was applied by measuring OD at nine different points of each well; however, a much higher resolution was applied this time in order to characterize colony dynamics and measuring points were individually considered. This study was performed on submerged and surface individual colonies after inoculating the wells with an initial level of 1-2 cells, which was achieved after performing the adequate decimal dilutions from the preculture. To obtain surface colonies, the spot inoculation method was carried out by pipetting a single drop from the preculture on the agar surface. OD measurements were performed with a FilterMax ${ }^{\mathrm{TM}}$ F5 microplate reader with MultiMode Analysis software at a wave length of $590 \mathrm{~nm}$. Results provided the necessary information to calculate the colony area by establishing 100 pixels as the minimum number to consider an object a colony. Authors reported a correspondence of this value with approximately $6 \log$ (CFU/colony). Through the scan method the evolution of the area of colonies was followed, which was linearly expanding with time and showed a similar trend between the independent colonies at early stages. At an individual level, an OD increment at the center of the colonies showed an increase in their height. The length of the experiments lasted for $23 \mathrm{~h}$, although this time is not the absolute limit of growth for a colony, the limited dimensions of the individual wells determined the experiments duration. The authors concluded that besides the limitations of the method, OD is an easy-to-use and flexible technique for monitoring bacterial colonies.

OD-based techniques provide many advantages for studying bacterial colonies, but there are still some limitations that it is necessary to overcome such us, the extended time required for the adequate data analysis, the uncertainty of the measured cell state (live, dead, sublethally injured, etc.), as well as some discrepancies between OD and viable count methods.

Another novel non-invasive technique to monitor bacterial colonies is based on microcalorimetric measurements. As defined by Braissant et al. (2010), isothermal calorimetry measures the heat flow of biological processes, which is proportional to the rate at which a chemical or physical process takes place. Microcalorimeters are the specific devices used in this field that measure heat flow of less than a micro-watt. The high sensitivity 
of the microcalorimeters favors their application in microbiology, since they can determine the heat released by bacterial cells while growing. This heat is represented as a function of time and the obtained curves are called calorimetric thermograms or powertime curves. Information gathered from this curves has been quantitatively correlated with biomass generation, changes in the number of cells, uptake oxygen, or substrates, etc., (Birou et al., 1987). This method presents several advantages for microbial studies, like the little preparation of the samples, non-invasive character (Braissant et al., 2010) and a continuous signal in real time. As a consequence, the applications of this method have been widely spread in different fields, more specifically for clinical (Trampuz et al., 2007), environmental and food microbiology (Gram and Sögaard, 1985; Alklint et al., 2005).

In the last years, microcalorimetric methods are drawing attention for studying bacterial colonies. The application of this technique to monitor and describe the growth of bacterial colonies in solid model systems, was presented for the first time by Kabanova et al. (2012). The main objective of this work was to assess the growth of individual colonies of L. lactis of different sizes. To obtain colonies of different sizes, serial inoculation levels were prepared from $10^{0}$ to $10^{6} \mathrm{CFU} / \mathrm{mL}$ by serially diluting the corresponding preculture. Colonies were grown in carbohydrate restricted medium (CMR) agar, which was initially inoculated and afterwards aseptically introduced in the specific ampoules for the microcalorimetric analysis. This analysis was carried out in a multi-channel thermal activity monitor TAM III, in which the heat is measured with a heat conduction calorimeter. From the resulting power-time curves different growth phases were identified, i.e., lag, exponential and deceleration or stationary phase (Kabanova et al., 2012, 2013). Maximum growth rate showed a dependency on the inoculum level, i.e., low inoculum levels led to higher values than those obtained from high inoculum levels. Although methods based on calorimetry allowed to study bacterial colonies non-invasively, the estimated growth dynamics gave information of the overall population. Thus, in Kabanova et al. (2012) the dimensions of the colonies were measured with a microscope Zeiss Axiovert $200 \mathrm{M}$ coupled with AxioCam MRc5 camera and Zeiss AxioVs40 V45.0.0 software. Colonies up to $10^{4}$ $\mathrm{CFU} / \mathrm{mL}$ showed a lenticular shape. This morphology was related to the agar gel structure and the size of the pores in which colonies grow. Once the colony grew over the pore size, the bonds between chains of the gel agar were broken, triggering a lenticular colony formation. Furthermore, this microscopically analysis revealed a homogeneous distribution of bacterial colonies in the media with the same characteristics as reported by Jeanson et al. (2011).

It can be concluded that microcalorimetry is a powerful technique to collect information of cell growth in solid (food) systems. Moreover, Kabanova et al. (2012) showed that in combination with other methods like the imaging-based ones a complete description of microbial behavior in solid (food) systems can be obtained.

\section{CONCLUSION}

Bacteria in solid (food) systems are known to grow forming colonies. This structured environment constraints cell mobility as well as nutrient and oxygen availability, giving rise to $\mathrm{pH}$ and oxygen gradients, due to diffusion limitations inside and around the colonies. Since studies of bacterial behavior have been mainly performed in liquid systems with planktonic cells, it comes of outmost importance to study colony growth dynamics in solid (food) systems, for an effective design of food safety assurance systems. Traditional techniques like viable plate counting, have widely contribute to increase the knowledge about bacterial growth in solid systems. However, these techniques provide information of the overall population but not of individual colonies and additionally, their invasive character requires the consequent sample discard. In order to overcome these disadvantages, new non-invasive techniques are being developed. Most of these novel techniques are based on processing microscopic images obtained from monitoring bacterial colony growth. From image processing, information regarding, e.g., morphology, the colony radius and colony area is gathered and related to bacterial growth. Other techniques based on OD measurements and microcalorimetry, provide information of colony appearance and evolution. Additionally, microcalorimetry has been shown to provide similar information to viable plate counting, thus, power-time curves can be used together with other measurements. Nevertheless, these techniques also show some disadvantages, i.e., the extended time required for data analysis with OD-based techniques and the limited number of experimental conditions that can be assessed with the gel-cassette, etc. In order to overcome these disadvantages, novel techniques are usually combined with viable plate counting. Further research is required to get more accurate knowledge about colony growth dynamics.

\section{ACKNOWLEDGMENTS}

This work was supported by project PFV/10/002 (Center of Excellence OPTEC-Optimization in Engineering) of the KU Leuven Research Council FWO-1518913N (KaN Multi-Objective Dyn. Opt.) project G.0930.13 of the Fund for Scientific ResearchFlanders, and the Belgian Program on Interuniversity Poles of Attraction, initiated by the Belgian Federal Science Policy Office (IAP Phase VII-19 DYSCO).

\section{REFERENCES}

Alklint, C., Wadsö, L., and Sjöholm, I. (2005). Accelerated storage and isothermal microcalorimetry as methods of predicting carrot juice shelf-life. J. Sci. Food Agr. 85, 281-285. doi: 10.1002/jsfa.1942

Antwi, M., Bernaerts, K., Van Impe, J. F., and Geeraerd, A. H. (2007). Modelling the combined effects of structured food model system and lactic acid on Listeria innocua and Lactococcus lactis growth in mono- and coculture. Int. J. Food Microbiol. 120, 71-84. doi: 10.1016/j.ijfoodmicro.2007.04.015

Antwi, M., Geeraerd, A. H., Vereecken, K., Jenne, R., Bernaerts, K., and Van Impe, J. F. (2006). Influence of a gel microstructure as modified by gelatin concentration on Listeria innocua growth. Innov. Food Sci. Emerg. Technol. 7, 124-131. doi: 10.1016/j.ifset.2005.08.001

Bae, E., Bai, N., Aroonnual, A., Bhunia, A. B., and Hirleman, E. D. (2010). Labelfree identification of bacterial microcolonies via elastic scattering. Biotechnol. Bioeng. 108, 637-644. doi: 10.1002/bit.22980

Baranyi, J., and Roberts, T. A. (1994). A dynamic approach to predicting bacterial growth in food. J. Food Microbiol. 23, 277-294. doi: 10.1016/0168-1605(94) 90157-0

Birou, B., Marison, I. W., and Von Stockar, U. (1987). Calorimetric investigation of aerobic fermentations. Biotechnol. Bioeng. 30, 650-660. doi: 10.1002/bit. 260300509

Boons, K., Noriega, E., Van de Broeck, R., David, C. C., Hofkens, J., and Van Impe, J. F. (2014). Effect of microstructure on population growth parameters of 
Escherichia coli in gelatin-dextran systems. Appl. Environ. Microbiol. 80, 53305339. doi: 10.1128/AEM.00817-14

Boons, K., Van der Linden, E., Mertens, L., Peeters, V., and Van Impe, J. F. (2013a). Effect of immobilization and salt concentration on the growth dynamics of Escherichia coli K12 and Salmonella Typhimurium. J. Food Sci. 78, 567-574. doi: 10.1111/1750-3841.12067

Boons, K., Mertens, L., Van der Linden, E., David, C. C., Hofkens, J., and Van Impe, J. F. (2013b). Behaviour of Escherichia coli in a heterogeneous gelatindextran mixture. Appl. Environ. Microbiol. 79, 3126-3128. doi: 10.1128/AEM. 03782-12

Braissant, O., Wirz, D., Goepfert, B., and Daniels, A. U. (2010). Use of isothermal microcalorimetry to monitor microbial activities. FEMS Microbiol. Lett. 303, 18. doi: 10.1111/j.1574-6968.2009.01819.x

Brocklehurst, T. F., Mitchell, G. A., Ridge, Y. P., Seale, R., and Smith, C. A. (1995). The effect of transient temperatures on the growth of Salmonella Typhimurium LT2 in gelatin gel. Int. J. Food Microbiol. 27, 45-60. doi: 10.1016/0168-1605(94) 00152-V

Dalgaard, P., and Koutsoumanis, K. P. (2000). Comparison of maximum specific growth rates and lag times estimated from absorbance and viable count data by different mathematical models. J. Microbiol. Methods 43, 183-196. doi: 10.1016/ S0167-7012(00)00219-0

Dalgaard, P., Ross, T., Kamperman, L., Neumeyer, K., and McMeekin, T. A. (1994). Estimation of bacterial growth rates from turbidimetric and viable count data. Int. J. Food Microbiol. 23, 391-404. doi: 10.1016/0168-1605(94)90165-1

Dens, E., and Van Impe, J. F. (2001). On the need for another type of predictive model in structured foods. Int. J. Food Microbiol. 64, 247-260. doi: 10.1016/ S0168-1605(00)00472-4

Dervakos, G. A., and Webb, C. (1991). On the merits of viable-cell immobilisation. Biotechnol. Adv. 9, 559-612. doi: 10.1016/0734-9750(91)90733-C

Fang, J., and Dorrestein, P. C. (2014). Emerging mass spectrometry techniques for the direct analyses of microbial colonies. Curr. Opin. Microbiol. 19, 120-129. doi: 10.1016/j.mib.2014.06.014

Floury, J., Jeanson, S., Madec, M. N., and Lortal, S. (2013). Porosity of Lactococcus lactis subsp lactis LD61 colonies immobilized in model cheese. Int. J. Food Microbiol. 163, 64-70. doi: 10.1016/j.ijfoodmicro.2013.02.014

Gonzalez, D. J., Xu, Y., Yang, Y. L., Esquenazi, E., Liu, W. T., Edlund, A., et al. (2012). Observing the invisible imaging mass spectrometry, a window into metabolic exchange patterns of microbes. J. Proteomics 75, 5069-5076. doi: 10.1016/j.jprot. 2012.05.036

Gram, L., and Sögaard, H. (1985). Microcalorimetry as a rapid method for estimation of bacterial levels in ground meat. J. Food Protect. 48, 341-345.

Guillier, L., Pardon, P., and Augustine, J. C. (2006). Automated image analysis of bacterial colony growth as a tool to study individual lag time distributions of immobilized cells. J. Microbiol. Methods 65, 324-334. doi: 10.1016/j.mimet. 2005.08.007

Helm, D., Labischinski, H., Schallehn, G., and Naumann, D. (1991). Classification and identification of bacteria by Fourier-transform infrared-spectroscopy. J. Gen. Microbiol. 137, 69-79. doi: 10.1099/00221287-137-1-69

Jeanson, S., Chadoeuf, J., Madec, M. N., Aly, S., Floury, J., Brocklehurst, T. F., et al. (2011). Spatial distribution of bacterial colonies in a model cheese. Appl. Environ. Microbiol. 77, 1493-1500. doi: 10.1128/AEM.02233-10

Jeanson, S., Floury, J., Issulahi, A. A., Madec, M. N., Thierry, A., and Lortal, S. (2013). Microgradients of $\mathrm{pH}$ do not occur around Lactococcus colonies in a model cheese. Appl. Environ. Microbiol. 79, 6516-6518. doi: 10.1128/AEM. 01678-13

Kabanova, N., Stulova, I., and Vilu, R. (2012). Microcalorimetric study of the growth of bacterial colonies of Lactococcus lactis IL1403 in agar gels. Food Microbiol. 29, 67-79. doi: 10.1016/j.fm.2011.08.018

Kabanova, N., Stulova, I., and Vilu, R. (2013). Microcalorimetric study of growth of Lactococcus lactis IL1403 at low glucose concentration in liquids and solid agar gels. Thermochim. Acta 59, 69-75. doi: 10.1016/j.tca.2013.02.013

Katsaras, K., and Leistner, L. (1988). Distribution and development of bacterial colonies in fermented sausages. Biofouling 5, 115-124. doi: 10.1080/ 08927019109378233

Kim, H., Singh, A. K., Bhunia, A. K., and Bae, E. (2014). Laser-induced speckle scatter patterns in Bacillus colonies. Front. Microbiol. 5:537. doi: 10.3389/fmicb. 2014.00537

Koch, A. L. (1981). "Growth measurements," in Manual of Methods for General Bacteriology, eds P. Gerhardt, R. G. E. Murray, R. N. Costilow, E. W. Nester,
W. A. Wood, N. R. Krieg, and G. R. Phillips (Washington, DC: American Society for Microbiology), 179-207.

Koutsoumanis, K. P., and Lianou, A. (2013). Stochasticity in colonial growth dynamics of individual bacterial cells. Appl. Environ. Microbiol. 79, 2294-2301. doi: 10.1128/AEM.03629-12

Malakar, P. K., Barker, G. C., Zwietering, M. H., and van’t Riet, K. (2003). Relevance of microbial interactions to predictive microbiology. Int. J. Food Microbiol. 84, 263-272. doi: 10.1016/S0168-1605(02)00424-5

Malakar, P. K., Brocklehurst, T. F., Mackie, A. R., Wilson, P. D. G., Zwietering, M. H., and van't Riet, K. (2000). Microgradients in bacterial colonies: use of fluorescence ratio imaging, a non-invasive technique. Int. J. Food Microbiol. 56, 71-80. doi: 10.1016/S0168-1605(00)00222-1

Malakar, P. K., Martens, D. E., van Breukelen, W., Boom, R. M., Zwietering, M. H., and van't Riet, K. (2002). Modeling interactions of Lactobacillus curvatus colonies in solid medium: consequences for food quality and safety. Appl. Environ. Microbiol. 68, 3432-3441. doi: 10.1128/AEM.68.7.3432-3441. 2002

McClure, P. J., Cole, M. B., Davies, K. W., and Anderson, W. A. (1993). The use of automated turbidimetric data for the construction of kinetic models. J. Industr. Microbiol. 12, 277-285. doi: 10.1007/BF01584203

Meldrum, R. J., Brocklehurst, T. F., Wilson, D. R., and Wilson, P. D. G. (2003). The effects of cell immobilization, $\mathrm{pH}$ and sucrose on the growth of Listeria monocytogenes Scott A at $10^{\circ} \mathrm{C}$. Food Microbiol. 20, 97-103. doi: 10.1016/S07400020(02)00083-7

Mertens, L., Geeraerd, A. H., Dang, T. D. T., Vermeulen, A., Sneerls, K., Van Derlinden, E., et al. (2009). Design of an experimental viscoelastic food model system for studying Zygosaccharomyces bailii spoilage in acidic sauces. Appl. Environ. Microbiol. 75, 7060-7069. doi: 10.1128/AEM.01045-09

Mertens, L., Van Derlinden, E., Dang, T. D. T., Cappuyns, A. M., Vermeulen, A., Debevere, J., et al. (2011). On the critical evaluation of growth/no growth assessment of Zygosaccharomyces bailii with optical density measurements: liquid versus structured media. Int. J. Food Microbiol. 28, 736-745. doi: 10.1016/ j.fm.2010.05.032

Mertens, L., Van Derlinden, E., and Van Impe, J. F. (2012). A novel method for high-throughput data collection in predictive microbiology: optical density monitoring of colony growth as a function of time. Food Microbiol. 32, 196-201. doi: 10.1016/j.fm.2012.04.001

Métris, A., George, S. M., and Baranyi, J. (2006). Use of optical density detection time to assess the effect of acetic acid on single-cell kinetics. Appl. Environ. Microbiol. 72, 6674-6679. doi: 10.1128/AEM.00914-06

Nerbrink, E., Borch, E., Blom, H., and Nesbakken, T. (1999). A model based on absorbance data on the growth rate of Listeria monocytogenes and including the effects of $\mathrm{pH}, \mathrm{NaCl}, \mathrm{Na}-$ lactate and $\mathrm{Na}$-acetate. Int. J. Food Microbiol. 47, 99-109. doi: 10.1016/S0168-1605(99)00021-5

Noriega, E., Laca, A., and Diaz, M. (2010). Decisive role of structure in food microbial colonization and implications for predictive microbiology. J. Food Protect. 73, 938-951.

Noriega, E., Velliou, E., Van Derlinden, E., Mertens, L., and Van Impe, J. F. (2013). Effect of cell immobilization on heat-induced sublethal injury of Escherichia coli, Salmonella Typhimurium and Listeria innocua. Food Microbiol. 36, 355-364. doi: 10.1016/j.fm.2013.06.015

Peters, A. C., Wimpenny, J. W. T., and Combs, J. P. (1987). Oxygen profiles in, and in the agar beneath, colonies of Bacillus cereus, Staphylococcus albus and Escherichia coli. J. Gen. Microbiol. 133, 1257-1263.

Rasch, M., Métris, A., Baranyi, J., and Bjørn Budde, B. (2007). The effect of reuterin on the lag time of single cells of Listeria innocua grown on a solid agar surface at different $\mathrm{pH}$ and $\mathrm{NaCl}$ concentrations. Int. J. Food Microbiol. 113, 35-40. doi: 10.1016/j.iffoodmicro.2006.07.012

Robins, M. M., and Wilson, P. D. G. (1994). Food structure and microbial growth. Trends. Food. Sci. Technol. 5, 289-293 doi: 10.1016/0924-2244(94) 90137-6

Robinson, T. P., Wimpenny, J. W. T., and Earnshaw, R. G. (1991). pH gradients through colonies of Bacillus cereus and the surrounding agar. J. Gen. Microbiol. 137, 2885-2889. doi: 10.1099/00221287-137-12-2885

Ross, T. (1996). Indices for performance evaluation of predictive models in food microbiology. J. Appl. Microbiol. 81, 501-508. doi: 10.1111/j.1365-2672.1996. tb01946.x

Skandamis, P., Tsigarida, E., and Nychas, G.-E. (2000). Ecophysiological attributes of Salmonella Typhimurium in liquid culture and within gelatin gel with or 
without the addition of oregano essential oil. World J. Microbiol. Biotechnol. 16, 31-35. doi: 10.1023/A:1008934020409

Skandamis, P. N., Brocklehurst, T. F., Panagou, E. Z., and Nychas, G.-J. E. (2007). Image analysis as a mean to model growth of Escherichia coli O157:H7 in gel cassettes. J. Appl. Microbiol. 103, 937-947. doi: 10.1111/j.1365-2672.2007. 03358.x

Tammam, J. D., Williams, A. G., Banks, J., Cowie, G., and Lloyd, D. (2001). Membrane inlet mass spectrometric measurements of $\mathrm{O}_{2}$ and $\mathrm{CO}_{2}$ gradients in cultures of Lactobacillus paracasei and developing Cheddar cheese ecosystem. Int. J. Food Microbiol. 65, 11-22. doi: 10.1016/S0168-1605(00)00438-4

Theys, T., Geeraerd, A., Devlieghere, F., and Van Impe, J. (2009). Extracting information on the evolution of living and dead cell fractions of Salmonella Typhimurium colonies in gelatin gels based on microscopic images and plate count data. Lett. Appl. Microbiol. 49, 39-45. doi: 10.1111/j.1472-765X.2009. 02623.x

Theys, T. E., Geeraerd, A. H., Verhulst, A., Poot, K., Van Bree, I., Devlieghere, F., et al. (2008). Effect of pH, water activity and gel micro-structure, including oxygen profiles and rheological characterization, on the growth kinetics of Salmonella Typhimurium. Int. J. Food Microbiol. 128, 67-77 doi: 10.1016/ j.ijfoodmicro.2008.06.031

Thi, N. N., Kirschner, C., and Naumann, D. (2003). Characterization and identification of microorganisms by FT-IR microspectrometry. J. Mol. Struct. 661, 371-380. doi: 10.1016/j.molstruc.2003.08.012

Thi, N. N., and Naumann, D. (2007). Investigating the heterogeneity of cell growth in microbial colonies by FTIR microspectroscopy. Anal. Bioanal. Chem. 387, 1769-1777. doi: 10.1007/s00216-006-0829-z

Trampuz, A., Salzmann, S., Antheaume, J., and Daniels, A. U. (2007). Microcalorimetry: a novel method for detection of microbial contamination in platelet products. Transfusion 547, 1643-1650. doi: 10.1111/j.1537-2995.2007.01336.x

Tsigarida, E., Boziaris, I. S., and George-John E. Nychas, G. J. E. (2003). Bacterial synergism or antagonism in a gel cassette system. Appl. Environ. Microbiol. 69, 7204-7209. doi: 10.1128/AEM.69.12.7204-7209.2003
Walker, S. L., Brocklehurst, T. F, and Wimpenny, J. W. T. (1997). The effect of growth dynamics upon $\mathrm{pH}$ gradient formation within and around subsurfaces of Salmonella Typhimurium. J. Appl. Microbiol. 82, 610-614. doi: 10.1111/j.13652672.1997.tb02869.x

Wilson, P. D. G., Brocklehurst, T. F., Arino, S., Thuault, D., Jakobsen, M., Lange, M., Farkas, J., et al. (2002). Modelling microbial growth in structured foods: towards a unified approach. Int. J. Food Microbiol. 73, 275-289. doi: 10.1016/S01681605(01)00660-2

Wimpenny, J., and Coombs, J. (1983). Penetration of oxygen into bacterial colonies. J. Gen. Microbiol. 129, 1239-1242.

Wimpenny, J. W. T., Leistner, L., Thomas, L. V., Mitchell, A. J., Katsaras, K., and Peetz, P. (1995). Submerged bacterial colonies within food and model systems: their growth, distribution and interactions. Int. J. Food Microbiol. 28, 299-315. doi: 10.1016/0168-1605(95)00065-8

Conflict of Interest Statement: The authors declare that the research was conducted in the absence of any commercial or financial relationships that could be construed as a potential conflict of interest.

Received: 30 September 2014; accepted: 09 February 2015; published online: 06 March 2015.

Citation: Lobete MM, Noriega Fernandez E and Van Impe JFM (2015) Recent trends in non-invasive in situ techniques to monitor bacterial colonies in solid (model) food. Front. Microbiol. 6:148. doi: 10.3389/fmicb.2015.00148

This article was submitted to Food Microbiology, a section of the journal Frontiers in Microbiology.

Copyright $\odot 2015$ Lobete, Noriega Fernandez and Van Impe. This is an open-access article distributed under the terms of the Creative Commons Attribution License (CC $B Y)$. The use, distribution or reproduction in other forums is permitted, provided the original author(s) or licensor are credited and that the original publication in this journal is cited, in accordance with accepted academic practice. No use, distribution or reproduction is permitted which does not comply with these terms. 


\title{
Growth and location of bacterial colonies within dairy foods using microscopy techniques: a review
}

\author{
Cian D. Hickey ${ }^{1,2}$, Jeremiah J. Sheehan ${ }^{1}$, Martin G. Wilkinson ${ }^{2}$ and Mark A. E. Auty ${ }^{1}$ * \\ 'Teagasc Food Research Centre, Fermoy, Ireland \\ 2 University of Limerick, Limerick, Ireland
}

\section{Edited by:}

Sophie Jeanson, Institut National de la Recherche Agronomique, France

\section{Reviewed by:}

Louis Coroller, Université de Brest France

IIkin Yucel Sengun, Ege University, Turkey

${ }^{*}$ Correspondence

Mark A. E. Auty, Teagasc Food Research Centre, Fermoy, Ireland e-mail: mark.auty@teagasc.ie
The growth, location, and distribution of bacterial colonies in dairy products are important factors for the ripening and flavor development of cheeses, yogurts, and soured creams. Starter, non-starter, spoilage, and pathogenic bacteria all become entrapped in the developing casein matrix of dairy foods. In order to visualize these bacterial colonies and the environments surrounding them, microscopy techniques are used. The use of various microscopy methods allow for the rapid detection, enumeration, and distribution of starter, non-starter and pathogenic bacteria in dairy foods. Confocal laser scanning microscopy is extensively utilized to identify bacteria location via the use of fluorescent dyes. Further study is needed in relation to the development of micro- gradients and localized ripening parameters in dairy products due to the location of bacteria at the protein-fat interface. Development in the area of bacterial discrimination using microscopy techniques and fluorescent dyes/tags is needed as the benefits of rapidly identifying spoilage/pathogenic bacteria early in product manufacture would be of huge benefit in relation to both safety and financial concerns.

Keywords: lactic acid bacteria, milk fermentation, bacterial location, cheese, microscopy, fat-protein interface

\section{BACTERIA WITHIN DAIRY PRODUCTS}

Bacteria are naturally present and are used extensively across all areas of dairy and food fermentation, either as natural microflora, or as starter cultures added under controlled conditions (Yang et al., 2012). Their fermentative ability, especially that of lactic acid bacteria (LAB) is based on the creation of an acidic environment through the breakdown of carbohydrates such as lactose, maltose, lactulose and sucrose thereby ensuring preservation of food stuffs. Fermented dairy products are often not manufactured under sterile conditions or with sterile milk (unpasteurized) and this can allow non-starter LAB as well as spoilage or pathogenic bacteria access to the fermenting food system (Montville and Matthews, 2005). LAB's commonly found in dairy products include strains of Streptococcus, Lactococcus, Lactobacilli, Bifidobacteria, Enterococcus, and Pediococci. Within these species there are numerous strain types which can be used in fermentation processes to give specific acidification and flavor profiles to the final product.

Bacteria associated with dairy fermentations can grow over a wide temperature range from 4 to $50^{\circ} \mathrm{C}$. Mesophilic bacteria have an optimum growth range of $25-35^{\circ} \mathrm{C}$, while thermophilic species have an optimum range of $37-45^{\circ} \mathrm{C}$ (Johnson and Steele, 2013). The growth of bacterial cells within dairy foods is heavily influenced by parameters such as $\mathrm{pH}$, water activity and salt-in-moisture levels as well as temperature.

The use of starter bacteria is needed in order to acidify the cheese milk before and during dairy food production. These starter bacteria are inoculated into the milk at their optimum growth temperature (described above) and then stored post manufacture at temperatures ranging from 4 to $12^{\circ} \mathrm{C}$ (depending on the type of product) in order to slow the growth and acidification of these bacteria. Adjunct cultures such as Propionibacterium become active via exposure to warmer temperature ranging from 20 to $25^{\circ} \mathrm{C}$ for a set period of time and are directly involved in the metabolism of lactate to propionic and acetic acid, water, and $\mathrm{CO}_{2}$ (Choisy et al., 2000; Hayaloglu and McSweeney, 2014).

\section{LACTIC ACID BACTERIA}

Lactic acid bacteria are the most common and important starter cultures used in fermented dairy products and may originate from the microflora of raw milks (e.g., bovine, ovine, caprine) but more frequently are inoculated intentionally during product manufacture. The initial role of $\mathrm{LAB}$ is to control $\mathrm{pH}$ of ripening milk and subsequent dairy products via the conversion of naturally occurring lactose found in milk to lactic acid (glycolysis). The rapid reduction (4-8 h) of $\mathrm{pH}$ to below 5.3 in cheese or 4.6 in fermented milk products allows for the control of non-starter microflora as only acid-tolerant bacteria can survive in those conditions (Johnson and Steele, 2013). The secondary function of LAB's in dairy fermentations is flavor development. Intracellular enzymes released by starter and non-starter bacteria during manufacture and ripening are the main contributors to flavor development via the three main biochemical pathways (glycolysis, lipolysis, and proteolysis). The breakdown of caseins is the most important pathway for flavor development in hard and semi- hard type cheese, which LAB contribute heavily to with the formation of small peptides and free amino acids which can then be further converted to form various alcohols, aldehydes, acids, and esters (Smit et al., 2005). Examples of dairy foods produced through LAB fermentations include cheeses, yogurts and sour creams such 
as crème fraiche. Examples of common starter LAB used in the dairy industry include Lactococcus lactis spp. lactis, L. lactis spp. cremoris (Cheddar), Lactobacillus helveticus, Lb. delbruecki spp. bulgaricus and Lb. casei (Swiss-/Italian-type cheese), Streptococcus thermophilus (Swiss-type cheese/yogurts) and Lb. acidophilus (yogurts, soured creams; Leroy and De Vuyst, 2004). These various starter cultures can often be used on their own or as part of a culture mix, incorporating positive aspects from various bacterial strains. Swiss type cheeses display the symbiotic role of mixed starter cultures. Lactose is converted to galactose and L- lactic acid by S. thermophilus and L. helveticus metabolizes this galactose to Land D- lactic acid as S. thermophilus is incapable of doing so (Fox et al., 1990).

Due to food safety concerns, commercially produced dairy products such as cheeses and yogurts are increasingly manufactured from pasteurized milk. Pasteurization inactivates pathogenic bacteria, but also results in a significant reduction, or inactivation of, naturally occurring microflora populations. Further control is achieved through competitive inhibition. Non-starter lactic acid bacteria (NSLAB) contribute toward flavor development in dairy foods. They are described as adventitious species which, in the case of dairy products, can originate from the factory environment or from the raw milk where they are present as adventitious contaminants (Crow et al., 2001). The most common NSLAB species found in the dairy industry are variants of Lactobacilli (Lactobacillus casei, L. paracasei, L. plantarum, L. curvatus, L. brevis, and L. fermentum) and common non-Lactobacillus species include Pediococcus acidilactici, $P$. pentosaceus, Enterococcus durans, E. faecalis, and E. faecium (Settanni and Moschetti, 2010).

While LAB account for the majority of bacteria present in dairy foods, either naturally occurring or through deliberate inoculation, other types of bacteria are also used within the dairy industry. These include adjunct cultures, defined as those added to fermented products for reasons other than acid formation (Giraffa, 2003), such as Staphylococci, Micrococci, Enterococci, and Propionibacterium (Chamba and Irlinger, 2004). The latter are used extensively in the manufacture of Swiss- type cheeses in order to produce the distinctive nutty flavor and metabolize lactate to acetate and carbon dioxide $\left(\mathrm{CO}_{2}\right)$ creating the characteristic eyes associated with these types of cheeses. Propionibacterium are responsible for high levels of lipolysis associated with Swisstype cheese and have been shown to have up to 100 times more lipolytic activity than LAB's (Chamba and Perreard, 2002; Chamba and Irlinger, 2004). Other adjunct culture types are not directly involved in cheese manufacture but become active only during ripening and include yeasts (Geotrichum candidum, Saccharomyces cerevisiae) and molds (Penicillium camemberti and P. roqueforti; Chamba and Irlinger, 2004).

\section{PATHOGENIC AND CONTAMINANT BACTERIA}

Bacteria may also be present in dairy foods as undesirable food spoilage or pathogenic agents. These undesirable bacteria may include psychrotophic bacteria (Pseudomonas fluorescens and $P$. putrefaciens) Listeria monocytogenes, Salmonella spp., Escherichia coli, Staphylococcus aureus, Clostridium botulinum, C. perfringens, C. tyrobutyricum, and Vibrio cholera (Giraffa, 2003; Oliver et al.,
2005; Gálvez et al., 2008; Machado et al., 2013). Proteases and lipases released by psychrotrophic bacteria in milk such as Pseudomonas fluorescens and P. putrefaciens can cause bitterness and off flavors in dairy products. These enzymes are heat stable and therefore unaffected by standard pasteurization temperatures (72$74^{\circ} \mathrm{C}$ for $15-30 \mathrm{~s}$ ), allowing for the development of off flavors in fermented dairy products (Sheehan, 2013).

The main pathogenic bacteria of concern in the dairy industry are those which are capable of surviving the manufacturing process of cheeses, yogurts and soured creams. L. monocytogenes is a gram positive bacterium responsible for causing gastroenteritis along with listeriosis, which in turn can cause serious illness through sepsis or meningitis. The fatality rate from extreme listeriosis ranges from 20 to 30\% (WHO, 2004; Carpentier and Cerf, 2011). E. coli is a gram negative bacterium which occurs naturally in the lower intestine but certain strains can cause gastroenteritis and urinary tract infections. E. coli in dairy products such as yogurts results from post pasteurization contamination. E. coli O157 has been shown to be able to survive the acidic conditions associated with yogurt manufacture thus causing serious health risks to consumers (Cirone et al., 2013). Salmonella spp. consists of several gram negative species each capable of causing food borne illness. Two of the most common found in dairy foods are S. typhimurium and S. enteritidis (Leyer and Johnson, 1992). S. aureus is a gram positive bacterium and one of the world's leading causes of food borne illness, such as gastroenteritis which is caused by the ingestion of enterotoxins produced by the bacteria. Therefore, even if the bacteria are killed via heat or pressure treatment, the heat resistant enterotoxin remains (Gálvez et al., 2008; Fleurot et al., 2014). The acidic environment created by the fermentation of dairy products, high levels of cleanliness and hygiene practice throughout manufacturing plants and correct storage of dairy products help prevent the growth and contamination of products with these undesirable pathogenic and food spoilage bacteria.

\section{BACTERIOCINS AND PROBIOTICS}

Bacteriocins are described as low-molecular mass proteins or peptides produced via bacterial ribosomal synthesis. They have an antimicrobial mode of action usually restricted to related Gram positive bacteria (Caplice and Fitzgerald, 1999; Leroy and De Vuyst, 2004). Bacteriocins are used as a mode of biopreservation. This is the extension of a food's storage life and increased food safety due the antibacterial products of natural or controlled microflora (Stiles, 1996; Gálvez et al., 2008). Many LAB are bacteriocin producers along with certain species of Enterococci (E. faecalis and E. faecium) which produce enteriocins (Giraffa, 2003). Examples of two commercially produced bacteriocins are nisin (Nisaplin in commercial form) which is produced by fermentation of milk based substrates by strains of Lactococcus lactis and Pediocin PA-1/AcH (ALTA 2341 in commercial form) which is produced from Pediococcus acidilactici (Gálvez et al., 2008). Nisin is used extensively throughout the dairy industry to prevent gas blowing in semi-hard and hard type cheeses caused by C. tyrobutyricum and as an antimicrobial agent in clotted and heat treated creams preventing the growth of Bacillus cereus even at low concentrations and in sliced cheeses it reduces the populations of 
Listeria innocula and S. aureus (Scannell et al., 2000; Gálvez et al., 2008).

Probiotics improve the nutritional value of a food and when administered in certain quantities provide health benefits to the host (Granato et al., 2010). Many LAB's display probiotic characteristics. The most common certified probiotic bacteria strains used in foods today consist of Gram-positive Lactobacillus and Bifidobacterium species, both of which are found heavily within the human gastrointestinal tract. The afore mentioned bacteria are linked to health benefits such as reduced lactose intolerance, relief from some diarrhoeas, lower blood cholesterol, increased immune response and preventions of certain cancers (Savadogo et al., 2006). The numbers of live bacteria reaching the gut is critical for probiotic efficacy and for this reason, quantifying and visualizing the bacterial colonies in a food prior to ingestion is very important.

\section{LOCATION OF BACTERIA}

The location of bacteria within dairy food matrices is of interest due to the possible effects these bacteria and their enzymes may have on ripening, particularly at the microscopic level in cheese and yogurts. The effect of bacterial colony size, distribution, and where colonies locate may all have an effect on the rate of enzyme release and localized ripening. In order to investigate these possibilities we need to visualize the behavior of developing bacterial colonies using microscopy techniques as discussed below. Bacterial distribution is not homogenous throughout dairy products as all types of bacterial cells (starter, non-starter, spoilage, pathogenic) become entrapped in the developing protein matrix resulting in random distribution of bacterial colonies (Fitzsimons et al., 2001). The immobilization of bacteria therefore requires the diffusion of substrates to the bacterial colony location and resulting metabolites must also diffuse through the protein matrix, resulting in the potential developments of micro-gradients in $\mathrm{pH}$ and water activity in and around the bacterial colonies (Floury et al., 2010). Initial studies suggest an interaction between milk fat globules and starter bacterial cells/colonies due to the regular location of bacterial colonies in close proximity or in direct contact with milk fat globules and their membranes (Laloy et al., 1996). Bacteria have since been shown to preferentially locate at the fat-protein interface and sometimes within whey pockets in dairy products.

\section{IMPORTANCE OF BACTERIA IN DAIRY FERMENTATIONS}

The growth of bacteria within fermenting food products is of extreme importance as this growth rate determines final cell numbers, acidification rates and thus the intensity of the fermentation process. Starter bacteria are also responsible for releasing intracellular enzymes upon death and subsequent lysis of the cell membrane. These enzymes catalyze a wide range of metabolic pathways (lipolysis, proteolysis, and glycolysis) which result in the formation of flavor compounds adding to flavor development in many cases of fermented foods (Wilkinson et al., 1994; Wilkinson and Kilcawley, 2005; Steele et al., 2013).

Each bacterial cell is believed to grow and form a colony within the food matrix after inoculation and in certain cases immobilization within the matrix (Jeanson et al., 2011). It is the activity, location, and environment of these colonies which are of interest in this review. To date a lot of research has been undertaken with relation to bacterial growth in various conditions in dairy foods. This review will focus on colony growth, location, and influence on the surrounding environment of starter, non-starter $\mathrm{LAB}$, spoilage and pathogenic bacteria in dairy products such as cheeses, sour creams and yogurts using microscopy to accurately quantify and visualize the bacteria in the food matrices.

\section{ANALYTICAL TECHNIQUES}

Methods for analysis of food can be separated into separate categories such as microbiological, microscopy, sensory, physical, and physico-chemical. These methods can be destructive or nondestructive in their procedure. For this review we will focus on the microscopy methods associated with dairy food analysis. Examples of non-destructive microscopic methods include confocal laser scanning microscopy (CLSM), cryo- and regular scanning electron microscopy (SEM), and transmission electron microscopy (TEM) which have helped to map the location and distribution of bacterial colonies in dairy foods.

Other rapid and reliable methods for enumerating and identifying bacterial cells which include polymerase chain reaction (PCR) amplification and its variants including PCR-denaturing gradient gel electrophoresis (PCR-DGGE) and PCR-temporal
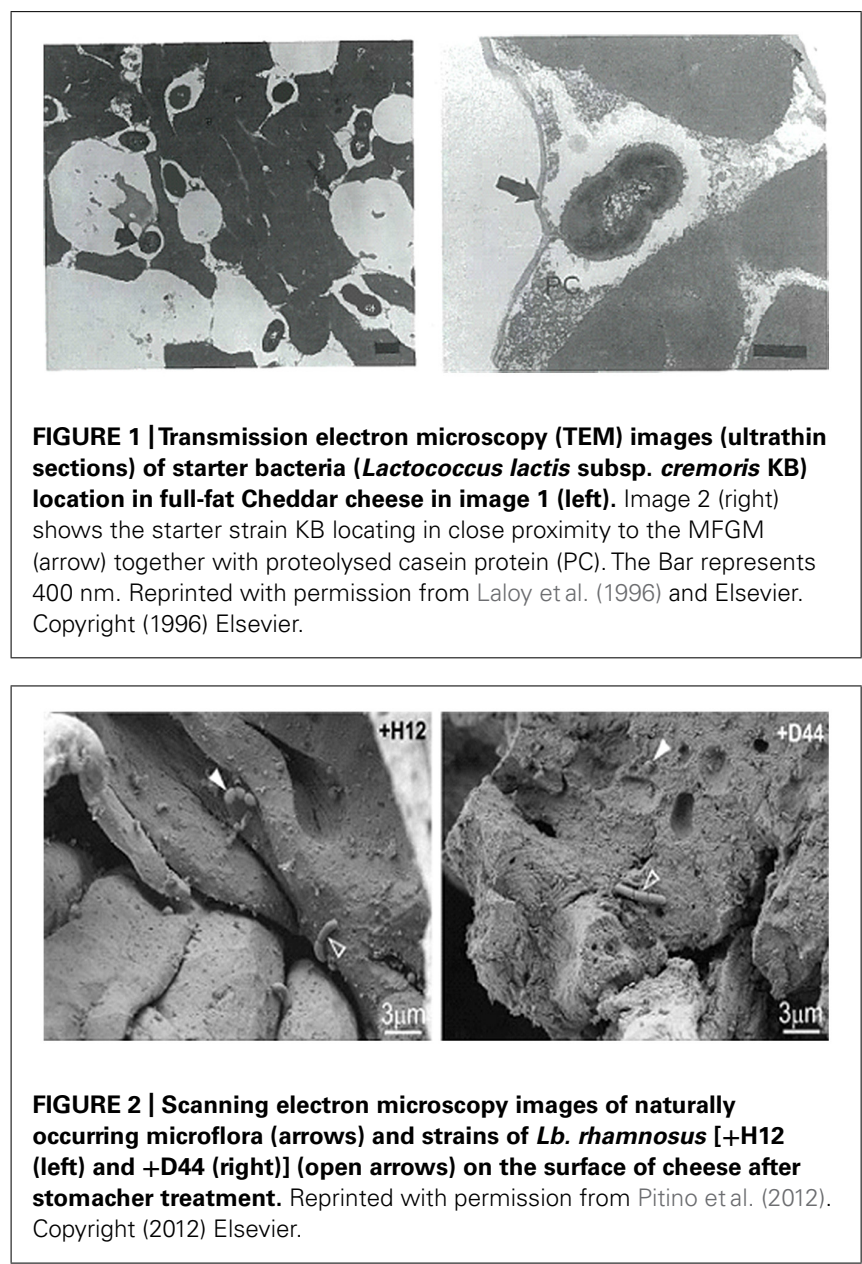


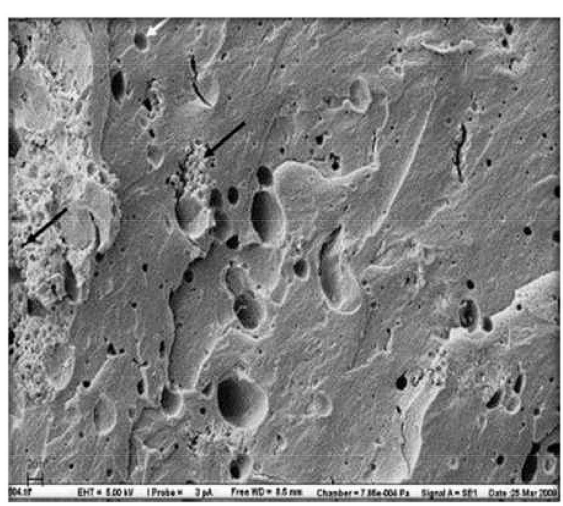

FIGURE 3 | Scanning electron microscopy (left) image of low-fat cheddar cheese matrix showing colonies of starter cultures (L. lactis) (black arrows) and areas previously occupied by fat globules (white arrows);

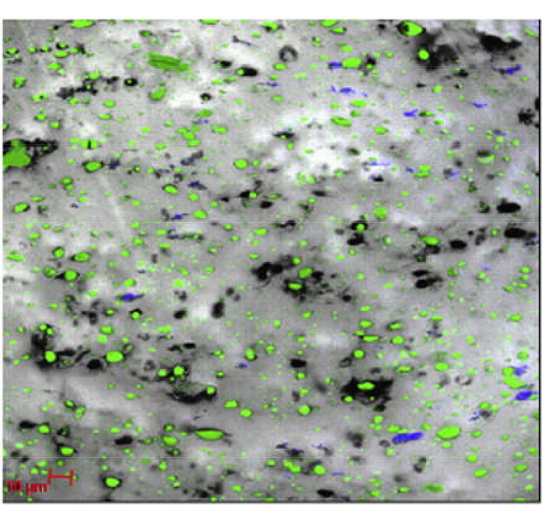

scale bar $=\mathbf{2 0} \boldsymbol{\mu} \mathbf{m}$. CLSM (right) image of cheese matrix with protein in gray, fat coded in green and bacterial colonies in blue; scale bar $=10 \mu \mathrm{m}$. Reprinted with permission from Romeih et al. (2012). Copyright (2012) Elsevier.

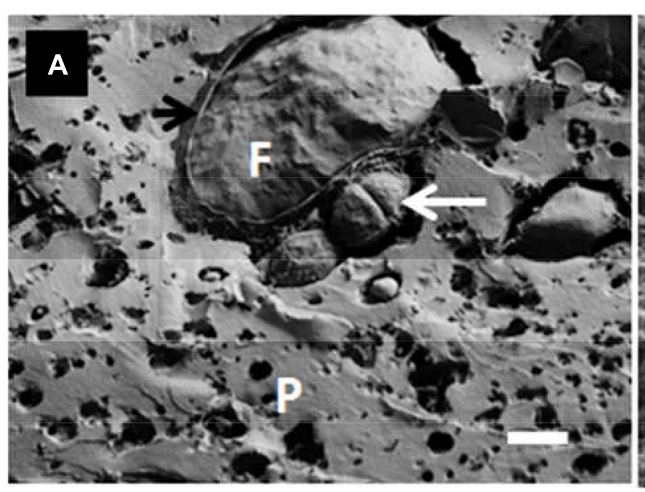

FIGURE 4 | (A) Cryo-SEM image of a Cheddar-type cheese showing the location of the starter bacteria ( $S$. thermophilus) (white arrow) and fat globule (F), including fractured MFGM (black arrow), within the protein network $(P)$ on day 12 of ripening; scale bar $=1 \mu \mathrm{m}$. (B) Cryo-SEM image

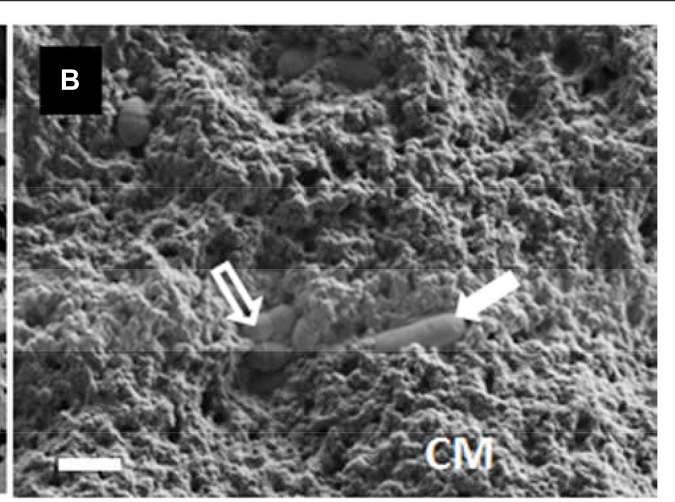

of starter bacteria in yogurt showing both $S$. thermophilus (open arrow) and Lb. delbruecki subsp. bulgaricus (closed arrow) entrapped within the acidified casein-based matrix $(\mathrm{CM})$; scale bar $=2 \mu \mathrm{m}$. Auty, unpublished results. temperature gradient gel electrophoresis (PCR-TTGE; Ndoye et al., 2011; Postollec et al., 2011; O'Sullivan et al., 2013). PCR targets a specific sequence such as the 16S rRNA gene in conjunction with genera/species specific primers. The $16 \mathrm{~S}$ rRNA gene is universal amongst bacteria and large databases exist for specific species of most food related bacteria. They have been used to determine the presence and quantity of undesirable bacteria such as C. tyrobutyricum and certain Lactobacillus communities in cheese. However, the issue with these methods of bacterial detection is the lack of information we obtain relating to bacterial colony location and distribution within dairy foods. For these reasons, this review focuses on microscopy methods allowing for the visualization of bacterial colony location and distribution.

\section{CONFOCAL LASER SCANNING MICROSCOPY}

The use of CLSM in food analysis has been at the forefront in recent years due to its ability to image individual components within a food matrix via the use of various fluorescent dyes (Auty et al., 2001, 2013; Romeih et al., 2012). This method is favored as a result of its ability to visualize thin optical sections below the surface of a sample due to the laser scanning function. CLSM uses argon and/or helium-neon lasers to individually obtain images from thin sections of sample which can then be stacked together in order to create a three dimensional (3D) image of a without disturbing the internal structure (De Freitas et al., 2007; Ong et al., 2011b; El-Bakry and Sheehan, 2014). Another advantage is its ability to analyze various components simultaneously via the use of fluorescent labels and stains, allowing for fat, protein, and bacterial colony location to be identified from one sample (Ong et al., 2010, 2011a, 2012, 2013b; Abhyankar et al., 2011). Commonly used fluorescent dyes in relation to dairy products and their components are fast green and rhodamine for the protein fractions, nile red for fats, and Oregon green 488/514 for determining localized $\mathrm{pH}$. Bacterial viability is commonly determined using a LIVE/DEAD BacLight viability kit consisting of two fluorescent nucleic acid stains SYTO9 (green) and propridium iodide (PI; red). SYTO9 permeates both viable and non-viable cell membranes, while PI only permeates damaged cell membranes which in turn negate the SYTO9 fluorescence. Thus, viable bacterial cells fluoresce green 


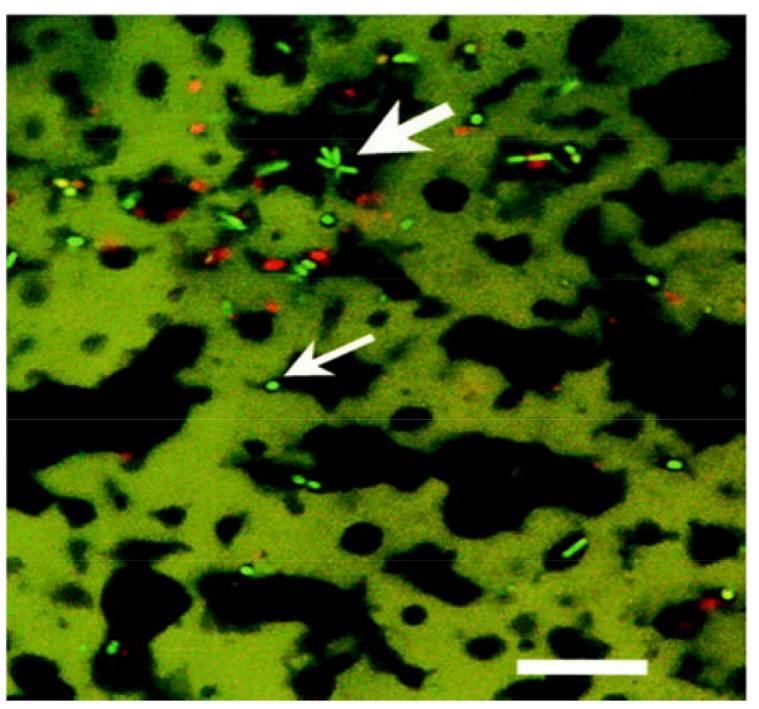

FIGURE 5 | Confocal laser scanning microscopy image of probiotic cheddar cheese showing star shaped clusters of live (bright green), presumptive Bifidobacteria, (large arrow), and dead (red) bacterial cells at fat (black)/protein (green) interface and presumptive NSLAB bacteria (small arrow). Scale bar $=25 \mu \mathrm{m}$. Reprinted with permission from Auty et al. (2001) and American Society for Microbiology (ASM).
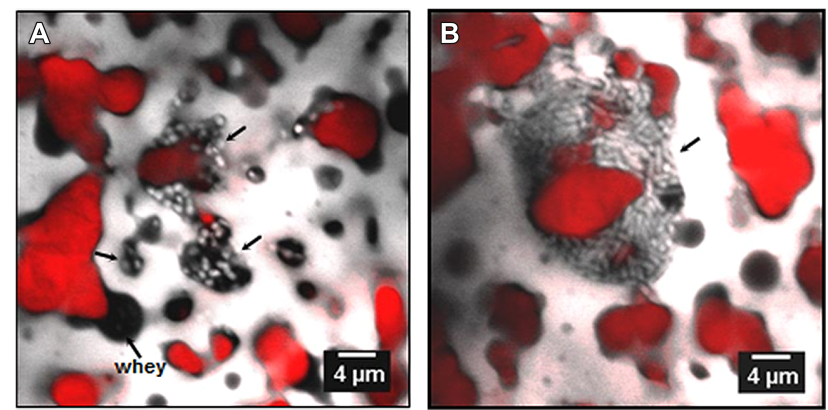

FIGURE 6 | Confocal laser scanning microscopy images of bacteria in Emmenal cheese after 1 day of ripening, showing location of bacterial colonies (light color) in whey pockets ( $A$, black areas) and at the interface (B) between fat (red) and protein (gray). Adapted from Lopez etal. (2006) with permission from the authors.

and those with a damaged or non-viable membrane fluoresce red (Auty et al., 2001). Bacterial location alone can be measured using Acridine orange which is a fluorescent dye which stains the DNA of bacteria (Lopez et al., 2006). This methodology has also been utilized recently to identify and track pathogenic bacterial growth in dairy foods (Fleurot et al., 2014).

\section{SCANNING ELECTRON MICROSCOPY}

Conventional SEM involves the generation of an electron beam which interacts with a given sample resulting in the emission of multiple secondary electrons. The image obtained is based on the electrons which scatter back when the electron beam strikes the surface of the sample (McMullan, 2006). In order to obtain a high number of secondary electrons and therefore give a clear image, a conducting layer is often placed over the sample surface to prevent charging (El-Bakry and Sheehan, 2014). The dehydration of the sample using a series of ethanol concentrations is necessary prior to examination, which must also be carried out under vacuum. This method has been used to study food microstructure for many years, offering a clear concise image of a samples surface showing fat, protein, and bacterial location (Pitino et al., 2012). The limitations associated with this methodology are the labor intensive sample preparation and the ability to only view the topographical area of a sample in addition to artifacts (Tunick et al., 2002).

Cryo-SEM comprises conventional SEM with a cryo-chamber attached allowing for the microscopic examination of dairy foods high in moisture, fat or air, i.e., cheese, yogurts, and soured creams. Operating at temperatures below $-80^{\circ} \mathrm{C}$, it utilizes liquid nitrogen in order to ultra-freeze samples. The advantages of this method over conventional SEM include a substantially reduced sample preparation time and a greater ability to view fat components which can become distorted due to the dehydration and defatting steps associated with conventional SEM. The use of this method has increased in recent years, in conjunction with techniques such as CLSM, for the study of dairy food microstructure and microbial population (Hassan et al., 2003; Romeih et al., 2012; Martinovic et al., 2013; Ong et al., 2013a).

\section{TRANSMISSION ELECTRON MICROSCOPY}

Transmission electron microscopy is similar to its scanning counterpart in that a beam of electrons are used but in this case the electrons pass through (transmit) the sample and the image generated is based on the scatter of these electrons, therefore samples need to be very thin $(0.1-0.2 \mu \mathrm{m})$. In relation to dairy analysis, replica type TEM is most commonly used. This involves either resin embedding and ultrathin sectioning or freeze-fractured replicas (Kaláb et al., 1995). Advantages include the best resolution of all electron based microscopy techniques allowing for greater examination of a samples ultra-structure (Laloy et al., 1996) and was first used on cheese by Green et al. (1981). Disadvantages consist of high cost, labor intensive sample preparation and possible presence of artifacts due to the use of osmium tetraoxide, which can cause fat and proteins to be misinterpreted due to inadequate fixing of the structures during sample preparation (Reis and Malcata, 2011; Auty et al., 2013; El-Bakry and Sheehan, 2014).

The use of these microscopy techniques in relation to bacterial location, survival and distribution in cheeses, yogurts and soured creams are discussed below in relation to the various studies which have been conducted on this important topic.

\section{LOCATION OF BACTERIAL COLONIES IN DAIRY PRODUCTS}

Location and distribution of various types of starter bacteria, NSLAB, contamination bacteria, and spoilage bacterial strains in a number of cheese varieties has been widely studied but rarely visualized using microscopic methods. (Hannon et al., 2006; Lopez et al., 2006, 2007; Jeanson et al., 2011). The ability to visualize the location of these bacteria within the developing protein matrix is of huge importance in relation to food quality, consistency and safety. 

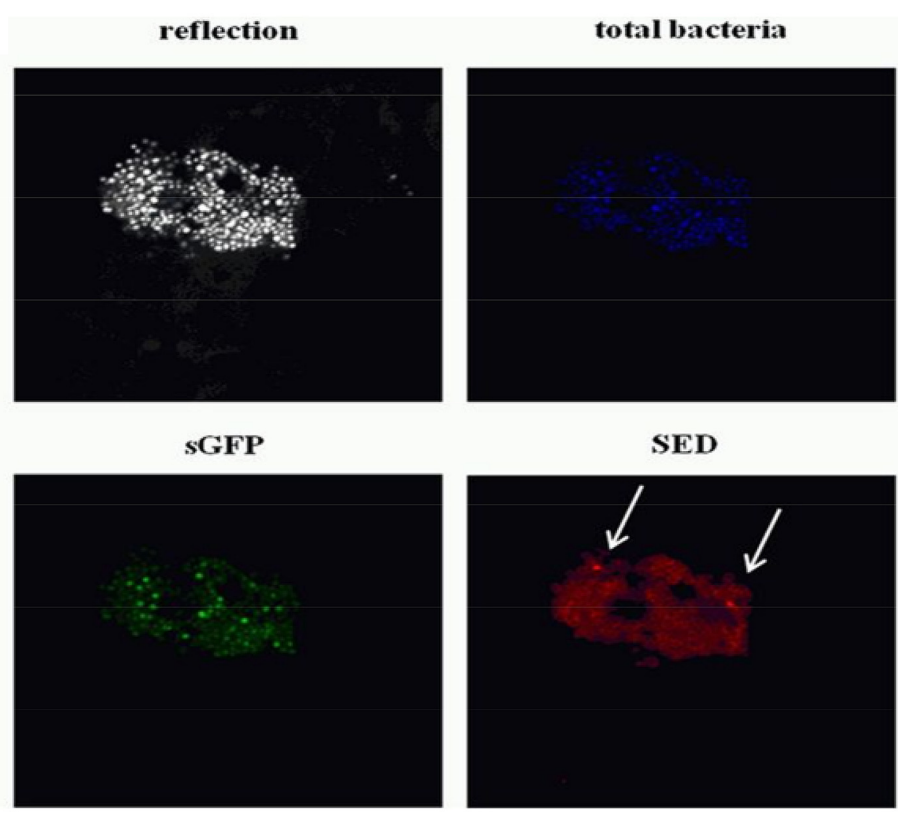

S. aureus

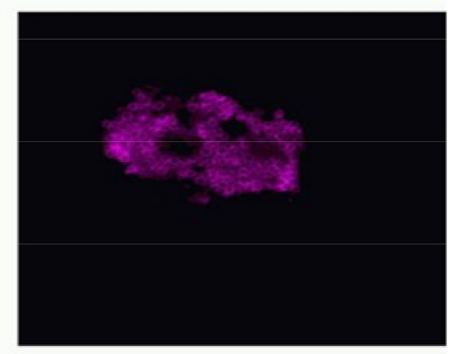

merge

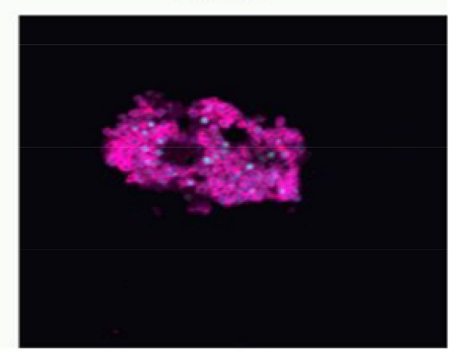

FIGURE 7|Confocal laser scanning microscopy images of dairy starter bacteria (Blue), synthetic green fluorescent protein (sGFP) (Green), Staphylococcal enterotoxin D (SED) (Red) and $S$. aureus (magenta) on the cheese surface 14 days into ripening. The cheese sample structure is visualized by the reflection of the 405-nm laser diode in a grayscale image. Reprinted (adapted) with permission from Fleurot etal. (2014). Copyright (2014) ASM.
Jeanson et al. (2011) studied bacterial distribution in a real food matrix (cheese) showing the spatial distribution of bacterial colonies at various levels of inoculation using CLSM. Bacterial colonies were shown to be randomly distributed which fit the proposed Poisson model. Their results supported the theory that increased inoculation levels $\left(10^{7} \mathrm{CFU} / \mathrm{g}\right)$ resulted in smaller colonies and displayed a sevenfold increase in the interfacial area of exchange with the cheese matrix compared to colonies formed at lower inoculation levels $\left(10^{4} \mathrm{CFU} / \mathrm{g}\right)$ as shown previously by McKay et al. (1997). Colonies can consist of bacterial cells in various physiological states of growth and McKay et al. (1997) have previously shown bacterial cells which are in the exponential phase of growth to be located on the colony exterior touching the matrix, most likely resulting in a high level of metabolic activity. This hypothesis allows for the assumption that the larger the interfacial area, the greater the bacterial activity on the food matrix which will in turn influence ripening. At higher levels of inoculation $\left(10^{6}\right.$ and $10^{7} \mathrm{cfu} / \mathrm{g}$ ) colonies were located extremely close together with mean distances of 25-30 $\mu \mathrm{m}$ between colonies (Jeanson et al., 2011).

Studies have been conducted into the possible location of bacterial micro-colonies within the cheese matrix. Laloy et al. (1996) used TEM to observe the location and distribution of starter bacteria in fat-free, $50 \%$ reduced fat and full fat Cheddar cheese (Figure 1). They found bacterial populations to be directly related to the fat content of the cheese. Compared to fat- free cheese, starter populations were $30-100 \%$ and $4-10$ fold higher in 50\% reduced fat and full fat cheese, respectively. Bacteria were found to be located in direct contact with the milk fat globular membrane (MFGM) or located at the casein-fat interface. As ripening progressed ( $>1-2$ months) bacteria seemed to become imbedded within or located inside the MFGM itself.

Pitino et al. (2012) utilized both SEM and CLSM in order to track the survival of $L b$. rhamnosus inoculated in cheese during simulated human digestion. They showed the interaction between Lb. rhamnosus and the cheese starter bacteria both of which appear to form colonies at the fat-protein interface or in contact with whey pockets (Figure 2).

Romeih etal. (2012) observed the location of L. lactis ssp. lactis ML8 using SEM in conjunction with CLSM (Figure 3), while Ong etal. (2013a) used cryo-SEM to detect the location of a mixture of mesophilic starter bacteria in full-fat Cheddar cheese. Auty (unpublished results) also used the same technique to show exopolysaccharide-producing lactic acid bacteria in Cheddar cheese and starter bacteria in yogurt, respectively, (Figure 4). As in the case of many others, the images appear to show preferential location of bacteria at the protein-fat interface. Images produced of yogurt show the entrapment of bacterial cells amongst the acidified casein-based matrix.

Auty etal. (2001), Hannon etal. (2006), and Lopez et al. (2006, 2007) used CLSM to visualize bacterial colonies in various cheeses such as Cheddar- type and Swiss- type cheeses made with ultrafiltered milk, respectively. In each case bacterial colonies were observed at the fat-protein interface also (Figures $\mathbf{5}$ and $\mathbf{6}$, respectively).

Fleurot et al. (2014) employed CLSM in order to locate the food borne pathogenic bacteria $S$. aureus in a cheese system. They found $S$. aureus formed colonies on the exterior surface of the cheese with colonies in the core a rarity, leading to the conclusion that $S$. aureus entrapped in the interior of the cheese did not multiply, 
while those on the aerated surface continued to multiply and form large colonies (Figure 7).

The application of microscopic techniques to investigate bacteria localization in dairy foods has been limited and in cases where it has been used, bacterial location is often observed as a result of investigating components such as fat content. Studies rarely focus on bacterial location and distribution specifically (Lopez et al., 2006,2007; Romeih et al., 2012; Ong et al., 2013a). Despite many of the aforementioned studies confirming that bacteria in dairy foods invariably locate on or in close proximity to the fat-protein interface or in contact with whey pockets, the effect this localization has on micro-gradients ( $\mathrm{pH}$, water activity), flavor development and overall product quality is still relatively unknown. Fleurot et al. (2014) is, to date, the only published work which uses CLSM in conjunction with fluorescent stains in order to discriminate between starter and pathogenic bacteria.

\section{CONCLUSION}

This review provides an overview on current information regarding use of microscopic techniques to investigate the growth and localization of bacteria within dairy based fermented foods. Such studies on bacterial colony location and distribution have not yet addressed the relationship between colony location and on product quality, consistency and on ripening parameters. However, the use of microscopy has made the visualization of bacteria in food matrices possible and allows for the enumeration, location, and distribution of starter LAB, NSLAB, spoilage, and pathogenic bacteria via non-destructive methods. Microscopy methods such as CLSM and cry-SEM allow for rapid sample analysis and CLSM allows for the detection of spoilage and pathogenic bacteria via the use of specific fluorescent dyes. Further study is greatly needed in this area regarding the influence bacterial location at the fat-protein interface has on localized micro-gradients and ripening parameters in a wide variety of dairy products. Microscopy allows for bacteria to be visualized within a solid food system and this tool is key to understanding bacterial behavior and influences on ripening fermented products. The discrimination of minority spoilage or pathogenic bacterial populations from the highly dense starter and non-starter dairy populations is an area which requires immediate attention as the benefits of possible rapid analysis and discrimination would be of great benefit to the entire food industry. Recent developments in superresolution optical microscopy and confocal Raman microscopy could be used in future to further characterize the microflora of solid foods and the localized biochemical transformations they influence.

\section{REFERENCES}

Abhyankar, A. R., Mulvihill, D. M., and Auty, M. A. E. (2011). Combined microscopic and dynamic rheological methods for studying the structural breakdown properties of whey protein gels and emulsion filled gels. Food Hydrocoll. 25, 275-282. doi: 10.1016/j.foodhyd.2010.05.012

Auty, M. A. E., Gardiner, G. E., Mcbrearty, S. J., O'sullivan, E. O., Mulvihill, D. M., Collins, J. K., et al. (2001). Direct in situ viability assessment of bacteria in probiotic dairy products using viability staining in conjunction with confocal scanning laser microscopy. Appl. Environ. Microbiol. 67, 420-425. doi: 10.1128/aem.67.1.420-425.2001

Auty, M., Morris, V., and Groves, K. (2013). "Confocal microscopy: principles and applications to food microstructures," Food Microstructures:
Microscopy, Measurement and Modelling, eds V. Morris and K. Groves (Cambridge, MA: Woodhead Publishing), 96-131. doi: 10.1533/978085709 8894.1.96

Caplice, E., and Fitzgerald, G. F. (1999). Food fermentations: role of microorganisms in food production and preservation. Int. J. Food Microbiol. 50, 131-149. doi: 10.1016/S0168-1605(99)00082-3

Carpentier, B., and Cerf, O. (2011). Review - Persistence of Listeria monocytogenes in food industry equipment and premises. Int. J. Food Microbiol. 145, 1-8. doi: 10.1016/j.ijfoodmicro.2011.01.005

Chamba, J. F., and Irlinger, F. (2004). "Secondary and adjunct cultures," in Cheese: Chemistry, Physics and Microbiology, Vol. 1, General Aspects, 3rd Edn., eds P. F. Fox, P. L. H. McSweeney, T. M. Cogan, and T. P. Guinee (London: Elsevier Academic Press), 191-206.

Chamba, J.-F., and Perreard, É. (2002). Contribution of propionic acid bacteria to lipolysis of Emmental cheese. Lait 82, 33-44. doi: 10.1051/lait:2001003

Choisy, C., Desmazeaud, M., Gueguen, M., Lenoir, J., Scmidt, J. L., and Tourneur, C. (2000). "Microbial phenomena," in Cheesmaking: From Science to Quality Assurance, 2nd Edn., eds A. Eck and J.-C. Gillis (Hampshire,: Intercept limited), 353-417.

Cirone, K., Huberman, Y., Morsella, C., Méndez, L., Jorge, M., and Paolicchi, F. (2013). Growth of Mycobacterium avium subsp. paratuberculosis, Escherichia coli, and Salmonella enteritidis during preparation and storage of yogurt. ISRN Microbiol. 247018, 7. doi: 10.1155/2013/247018

Crow, V., Curry, B., and Hayes, M. (2001). The ecology of non-starter lactic acid bacteria (NSLAB) and their use as adjuncts in New Zealand Cheddar. Int. Dairy J. 11, 275-283. doi: 10.1016/S0958-6946(01)00057-7

De Freitas, I., Pinon, N., Thierry, A., Lopez, C., Maubois, J.-L., and Lortal, S. (2007). In depth dynamic characterisation of French PDO Cantal cheese made from raw milk. Lait 87, 97-117. doi: 10.1051/lait:2007007

El-Bakry, M., and Sheehan, J. (2014). Analysing cheese microstructure: a review of recent developments. J. Food Eng. 125, 84-96. doi: 10.1016/j.jfoodeng.2013.10.030

Fitzsimons, N., Cogan, T., Condon, S., and Beresford, T. (2001). Spatial and temporal distribution of non-starter lactic acid bacteria in Cheddar cheese. J. Appl. Microbiol. 90, 600-608. doi: 10.1046/j.1365-2672.2001.01285.x

Fleurot, I., Aigle, M., Fleurot, R., Darrigo, C., Hennekinne, J.-A., Gruss, A., et al. (2014). Following pathogen development and gene expression in a food ecosystem: the case of a Staphylococcus aureus isolate in cheese. Appl. Environ. Microbiol. 80, 5106-5115. doi: 10.1128/aem.01042-14

Floury, J., Jeanson, S., Aly, S., and Lortal, S. (2010). Determination of the diffusion coefficients of small solutes in cheese: a review. Dairy Sci. Technol. 90, 477-508. doi: $10.1051 / \mathrm{dst} / 2010011$

Fox, P., Lucey, J., and Cogan, T. (1990). Glycolysis and related reactions during cheese manufacture and ripening. Crit. Rev. Food Sci. Nutr. 29, 237-253. doi: 10.1080/10408399009527526

Gálvez, A., López, R. L., Abriouel, H., Valdivia, E., and Omar, N. B. (2008). Application of bacteriocins in the control of foodborne pathogenic and spoilage bacteria. Crit. Rev. Biotechnol. 28, 125-152. doi: 10.1080/073885508021 07202

Giraffa, G. (2003). Functionality of enterococci in dairy products. Int. J. Food Microbiol. 88, 215-222. doi: 10.1016/S0168-1605(03)00183-1

Granato, D., Branco, G. F., Cruz, A. G., Faria, J. D. A. F., and Shah, N. P. (2010). Probiotic dairy products as functional foods. Compr. Rev. Food Sci. Food Saf. 9, 455-470. doi: 10.1111/j.1541-4337.2010.00120.x

Green, M. L., Turvey, A., and Hobbs, D. G. (1981). Development of structure and texture in cheddar cheese. J. Dairy Res. 48, 343-355. doi: 10.1017/S0022029900021774

Hannon, J., Lopez, C., Madec, M.-N., and Lortal, S. (2006). Altering Renneting pH changes microstructure, cell distribution, and lysis of $<\mathrm{i}>$ Lactococcus lactis $</ \mathrm{i}>$ AM2 in cheese made from ultrafiltered milk. J. Dairy Sci. 89, 812-823. doi: 10.3168/jds.S0022-0302(06)72144-0

Hassan, A. N., Frank, J. F., and Elsoda, M. (2003). Observation of bacterial exopolysaccharide in dairy products using cryo-scanning electron microscopy. Int. Dairy J. 13, 755-762. doi: 10.1016/S0958-6946(03) 00101-8

Hayaloglu, A., and McSweeney, P. (2014). "Primary biochemical events during cheese ripening," in Dairy Microbiology and Biochemistry: Recent Developments, eds O. Barbaros and A.-E. Gülsün (Boca Raton, FL: CRC Press, Taylor \& Francis), 134-166. doi: 10.1201/b17297-8 
Jeanson, S., Chadæuf, J., Madec, M., Aly, S., Floury, J., Brocklehurst, T., et al. (2011). Spatial distribution of bacterial colonies in a model cheese. Appl. Environ. Microbiol. 77, 1493-1500. doi: 10.1128/AEM.02233-10

Johnson, M., and Steele, J. (2013). "Fermented dairy products," in Food Microbiology: Fundamentals and Frontiers, eds M. P. Doyle and R. L. Buchanan (Washington, DC: ASM Press), 581-594.

Kaláb, M., Allan-Wojtas, P., and Miller, S. S. (1995). Microscopy and other imaging techniques in food structure analysis. Trends Food Sci. Tech. 6, 177-186. doi: 10.1016/S0924-2244(00)89052-4

Laloy, E., Vuillemard, J.-C., El Soda, M., and Simard, R. E. (1996). Influence of the fat content of Cheddar cheese on retention and localization of starters. Int. Dairy J. 6, 729-740. doi: 10.1016/0958-6946(95)00068-2

Leroy, F., and De Vuyst, L. (2004). Lactic acid bacteria as functional starter cultures for the food fermentation industry. Trends Food Sci. Technol. 15, 67-78. doi: 10.1016/j.tifs.2003.09.004

Leyer, G. J., and Johnson, E. A. (1992). Acid adaptation promotes survival of Salmonella spp. in cheese. Appl. Environ. Microbiol. 58, 2075-2080.

Lopez, C., Camier, B., and Gassi, J.-Y. (2007). Development of the milk fat microstructure during the manufacture and ripening of Emmental cheese observed by confocal laser scanning microscopy. Int. Dairy J. 17, 235-247. doi: 10.1016/j.idairyj.2005.12.015

Lopez, C., Maillard, M.-B., Briard-Bion, V., Camier, B., and Hannon, J. A. (2006). Lipolysis during ripening of Emmental cheese considering organization of fat and preferential localization of bacteria. J. Agric. Food Chem. 54, 5855-5867. doi: 10.1021/jf0602141

Machado, S. G., Bazzolli, D. M. S., and Vanetti, M. C. D. (2013). Development of a PCR method for detecting proteolytic psychrotrophic bacteria in raw milk. Int. Dairy J. 29, 8-14. doi: 10.1016/j.idairyj.2012.09.007

Martinovic, A., Moe, K. M., Romeih, E., Aideh, B., Vogensen, F. K., Østlie, H., et al. (2013). Growth of adjunct Lactobacillus casei in Cheddar cheese differing in milk fat globule membrane components. Int. Dairy J. 31, 70-82. doi: 10.1016/j.idairyj.2013.02.009

McKay, A. L., Peters, A. C., and Wimpenny, J. W. T. (1997). Determining specific growth rates in different regions of Salmonella typhimurium colonies. Lett. Appl. Microbiol. 24, 74-76. doi: 10.1046/j.1472-765X.1997.00354.x

McMullan, D. (2006). Scanning electron microscopy 1928-1965. Scanning 17, 175185. doi: 10.1002/sca.4950170309

Montville, T. J., and Matthews, K. R. (2005). "Fermentative organisms," in Food Microbiology: An Introduction, eds T. J. Montville and K. R. Matthews (Washington, DC: ASM Press), 223-239.

Ndoye, B., Rasolofo, E., Lapointe, G., and Roy, D. (2011). A review of the molecular approaches to investigate the diversity and activity of cheese microbiota. Dairy Sci. Technol. 91, 495-524. doi: 10.1007/s13594-011-0031-8

Oliver, S. P., Jayarao, B. M., and Almeida, R. A. (2005). Foodborne pathogens in milk and the dairy farm environment: food safety and public health implications. Foodbourne Pathog. Dis. 2, 115-129. doi: 10.1089/fpd.2005.2.115

Ong, L., Dagastine, R., Auty, M. E., Kentish, S., and Gras, S. (2011a). Coagulation temperature affects the microstructure and composition of full fat Cheddar cheese. Dairy Sci. Technol. 91, 739-758. doi: 10.1007/s13594-011-0033-6

Ong, L., Dagastine, R. R., Kentish, S. E., and Gras, S. L. (2011b). Microstructure of milk gel and cheese curd observed using cryo scanning electron microscopy and confocal microscopy. LWT Food Sci. Technol. 44, 1291-1302. doi: 10.1016/j.lwt.2010.12.026

Ong, L., Dagastine, R. R., Kentish, S. E., and Gras, S. L. (2010). The effect of milk processing on the microstructure of the milk fat globule and rennet induced gel observed using confocal laser scanning microscopy. J. Food Sci. 75, E135-E145. doi: 10.1111/j.1750-3841.2010.01517.x

Ong, L., Dagastine, R. R., Kentish, S. E., and Gras, S. L. (2012). The effect of pH at renneting on the microstructure, composition and texture of Cheddar cheese. Food Res. Int. 48, 119-130. doi: 10.1016/j.foodres.2012.02.020

Ong, L., Dagastine, R., Kentish, S., and Gras, S. (2013a). Microstructure and composition of full fat Cheddar cheese made with ultrafiltered milk retentate. Foods 2, 310-331. doi: 10.3390/foods2030310

Ong, L., Dagastine, R. R., Kentish, S. E., and Gras, S. L. (2013b). The effect of calcium chloride addition on the microstructure and composition of Cheddar cheese. Int. Dairy J. 33, 135-141. doi: 10.1016/j.idairyj.2013.03.002
O’Sullivan, D. J., Giblin, L., Mcsweeney, P. L., Sheehan, J. J., and Cotter, P. D. (2013). Nucleic acid-based approaches to investigate microbial-related cheese quality defects. Front. Microbiol. 4:1. doi: 10.3389/fmicb.2013.00001

Pitino, I., Randazzo, C. L., Cross, K. L., Parker, M. L., Bisignano, C., Wickham, M. S., et al. (2012). Survival of $<\mathrm{i}>$ Lactobacillus rhamnosus $</ \mathrm{i}>$ strains inoculated in cheese matrix during simulated human digestion. Food Microbiol. 31, 57-63. doi: 10.1016/j.fm.2012.02.013

Postollec, F., Falentin, H., Pavan, S., Combrisson, J., and Sohier, D. (2011). Recent advances in quantitative PCR (qPCR) applications in food microbiology. Food Microbiol. 28, 848-861. doi: 10.1016/j.fm.2011.02.008

Reis, P., and Malcata, F. (2011). Ripening-related changes in Serra da Estrela cheese: a stereological study. J. Dairy Sci. 94, 1223-1238. doi: 10.3168/jds.2010-3416

Romeih, E. A., Moe, K. M., and Skeie, S. (2012). The influence of fat globule membrane material on the microstructure of low-fat Cheddar cheese. Int. Dairy J. 26, 66-72. doi: 10.1016/j.idairyj.2012.03.008

Savadogo, A., Ouattara, A. C., Bassole, H. I., and Traore, S. A. (2006). Bacteriocins and lactic acid bacteria-a minireview. Afr. J. Biotechnol. 5, 678-683.

Scannell, A. G., Hill, C., Ross, R., Marx, S., Hartmeier, W., and Arendt, E. K. (2000). Development of bioactive food packaging materials using immobilised bacteriocins Lacticin 3147 and Nisaplin $<$ sup $>{ }^{\circledR}</$ sup $>$. Int. J. Food Microbiol. 60, 241-249. doi: 10.1016/S0168-1605(00)00314-7

Settanni, L., and Moschetti, G. (2010). Non-starter lactic acid bacteria used to improve cheese quality and provide health benefits. Food Microbiol. 27, 691-697. doi: 10.1016/j.fm.2010.05.023

Sheehan, J. J. (2013). Milk quality and cheese diversification. Irish J. Agric. Food Res. 52, 243-253.

Smit, G., Smit, B. A., and Engels, W. J. M. (2005). Flavour formation by lactic acid bacteria and biochemical flavour profiling of cheese products. FEMS Microbiol. Rev. 29, 591-610. doi: 10.1016/j.fmrre.2005.04.002

Steele, J., Broadbent, J., and Kok, J. (2013). Perspectives on the contribution of lactic acid bacteria to cheese flavor development. Curr. Opin. Biotechnol. 24, 135-141. doi: 10.1016/j.copbio.2012.12.001

Stiles, M. (1996). Biopreservation by lactic acid bacteria. Antonie Van Leeuwenhoek 70, 331-345. doi: 10.1007/BF00395940

Tunick, M. H., Van Hekken, D. L., Cooke, P. H., and Malin, E. L. (2002). Transmission electron microscopy of mozzarella cheeses made from microfluidized milk. J. Agric. Food Chem. 50, 99-103. doi: 10.1021/jf010633c

WHO. (2004). "Risk assessment of Listeria monocytogenes in ready-to-eat foods," in MRA Series, ed. World Health Orginization (Rome: WHO).

Wilkinson, M. G., Guinee, T. P., and Fox, P. F. (1994). Factors which may influence the determination of autolysis of starter bacteria during cheddar cheese ripening. Int. Dairy J. 4, 141-160. doi: 10.1016/0958-6946(94)90065-5

Wilkinson, M. G., and Kilcawley, K. N. (2005). Mechanisms of incorporation and release of enzymes into cheese during ripening. Int. Dairy J. 15, 817-830. doi: 10.1016/j.idairyj.2004.08.021

Yang, E., Fan, L., Jiang, Y., Doucette, C., and Fillmore, S. (2012). Antimicrobial activity of bacteriocin-producing lactic acid bacteria isolated from cheeses and yogurts. AMB Express 2, 1-12. doi: 10.1186/2191-0855-2-48

Conflict of Interest Statement: The authors declare that the research was conducted in the absence of any commercial or financial relationships that could be construed as a potential conflict of interest.

Received: 08 October 2014; accepted: 26 January 2015; published online: 18 February 2015.

Citation: Hickey CD, Sheehan JJ, Wilkinson MG and Auty MAE (2015) Growth and location of bacterial colonies within dairy foods using microscopy techniques: a review. Front. Microbiol. 6:99. doi: 10.3389/fmicb.2015.00099

This article was submitted to Food Microbiology, a section of the journal Frontiers in Microbiology.

Copyright (c) 2015 Hickey, Sheehan, Wilkinson and Auty. This is an open-access article distributed under the terms of the Creative Commons Attribution License (CC BY). The use, distribution or reproduction in other forums is permitted, provided the original author(s) or licensor are credited and that the original publication in this journal is cited, in accordance with accepted academic practice. No use, distribution or reproduction is permitted which does not comply with these terms. 


\section{In situ examination of Lactobacillus brevis after exposure to an oxidizing disinfectant}

\section{Yu Zhao, Susanne Knøchel and Henrik Siegumfeldt*}

Food Microbiology, Department of Food Science, Faculty of Science, University of Copenhagen, Frederiksberg C, Denmark

\section{Edited by:}

Sophie Jeanson, Institut National de la Recherche Agronomique, France

\section{Reviewed by:}

Carmen Wacher, Universidad Nacional Autónoma de México, Mexico

Laurent Guillier, Agence Nationale de Sécurité Sanitaire de l'Alimentation, de l' Environnement et du Travail, France

\section{*Correspondence:}

Henrik Siegumfeldt, Food Microbiology, Department of Food Science, Faculty of Science, University of Copenhagen, Rolighedsvej 26, 1958

Frederiksberg C, Denmark e-mail: siegum@food.ku.dk
Beer is a hostile environment for most microorganisms, but some lactic acid bacteria can grow in this environment. This is primarily because these organisms have developed the ability to grow in the presence of hops. It has been speculated that hop resistance is inversely correlated to resistance against oxidation, and this would have great impact on the use of various disinfectants in the brewing industry. In this study, we cultivated bacteria under aerobic and anaerobic conditions, and then investigated the in situ outgrowth of individual cells into microcolonies on de Man Rogosa Sharpe (MRS) agar after exposure to the oxidizing agent peracetic acid (PAA). An automated microscope stage allowed us to analyse a much larger number of cells over extended periods of incubation. After PAA treatment, the lag time increased markedly, and extensive variation in morphology, $\mu_{\max }$ as well as stress resistance was observed between and within the tested Lactobacillus brevis strains. The results suggest that aerobic cultivation increased the oxidative stress tolerance in Lactobacillus brevis. The results also show that dead cells are randomly distributed in a microcolony and the majority of non-growing individual cells do not stain with a membrane impermanent dye (Propidium iodide), which indicates that PAA may not destroy the plasma membrane. In conclusion, the developed microscopic analysis of individual cells on MRS agar can provides faster results and more details of cell physiology compared to the traditional CFU method.

Keywords: Lactobacillus brevis, aerobic cultivation, anaerobic cultivation, peracetic acid, solid surface, microscopic method, heterogeneity

\section{INTRODUCTION}

Lactic acid bacteria (LAB) are fermentative organisms which have been generally regarded as anaerobic bacteria, but most of them can grow under aerobic conditions (Sakamoto and Komagata, 1996). Beer is a relatively hostile medium for most microorganisms. The low $\mathrm{pH}$ will prevent most Gram-negative bacteria from growing, and the addition of hops will usually prevent LAB from spoiling the beer (Vaughan et al., 2005; Suzuki et al., 2006; Menz et al., 2010; Suzuki, 2011). However, some LAB possess a level of hop resistance, and therefore also possess the ability to spoil beer. $60-90 \%$ of bacteria isolated from spoiled beer are LAB (Suzuki, 2011). Among those LAB, Lactobacillus brevis is the most common bacteria and frequently detected in breweries (Hollerová and Kubizniaková, 2001; Suzuki et al., 2008a; Menz et al., 2010).

Recently, it has been suggested that hop resistance in LAB is inversely correlated to resistance toward oxidative compounds (Behr and Vogel, 2010). Consequently, it would be interesting to investigate the response of beer spoilage isolates toward oxidative compounds, as some of these are utilized as sanitizers in the food industry (Rossoni and Gaylarde, 2000; Kitis, 2004).

Another potential challenge is the relatively slow growth of the beer spoilage organisms. This means that often they are not detected readily in various culture media (Suzuki et al., 2008b). One way of facilitating the detection would be to use detection of growth of individual cells into micro-colonies, as the formations of macrocolonies require a longer incubation period in traditional CFU method. Recently, some studies have developed bioimaging methods for detecting the growth of individual cells in/on a solid matrix. Elfwing et al. (2004) designed a flow chamber microscopic method to observe growth and proliferation of single cells of Escherichia coli and Listeria innocua. Niven et al. (2006) developed a phase-contrast microscopy method to determine the first division time and individual lag times on agar media. Mertens et al. (2012) studied the colony growth dynamics based on optical density measurements on solid medium in microtiter plates. Koutsoumanis and Lianou (2013) used timelapse microscopy videos to count the cells and to observe the division of Salmonella single cells directly on agar media. Ryssel et al. (2013) developed a microscopy method to monitor growth and death of individual Lactococcus lactis cells based on staining with propidium iodide (PI) in the agar media. In addition, another advantage of investigating individual cells growing on a solid substrate is the ability to analyze the heterogeneity of a given population, as each individual cell gives rise to a unique microcolony.

The current study therefore investigates the impact of oxidizing substances on the survival of beer spoilage LAB. The study describes an automated image-acquisition microscopic method that enables the analysis of growth as well as the death of individual cells while growing on the surface of a semisolid substrate. 


\section{MATERIALS AND METHODS}

\section{BACTERIAL STRAINS AND GROWTH CONDITIONS}

The strains of Lactobacillus brevis used in this study are listed in Table 1. All experiments were initiated by inoculating $10 \mathrm{ml}$ de Man Rogosa Sharpe (MRS) broth (Merck, pH 5.7) from a frozen stock culture, followed by incubation at $30^{\circ} \mathrm{C}$ overnight. Subsequently, $100 \mu \mathrm{l}$ culture was subcultured into $10 \mathrm{ml}$ fresh MRS broth. For aerobic cultivation, the tubes was shaken around $300 \mathrm{rpm}$ at $30^{\circ} \mathrm{C}$. For anaerobic cultivation, the tubes were incubated at $30^{\circ} \mathrm{C}$ in an anaerobic jar, and incubated until an approximate $\mathrm{OD}_{600}$ value of 1.5 . The cultures were subsequently exposed to oxidizing agents as described below.

\section{TREATMENTS OF LAB WITH OXIDIZING AGENTS}

Two kinds of disinfectants were used in this study: peracetic acid (PAA, Sigma, 101272695) and sodium hypochlorite ( $\mathrm{NaClO}$, Sigma, 101292621). The final concentration of PAA solution during exposure was $0.0014 \%$, and the final concentration of $\mathrm{NaClO}$ solution was $0.0021 \%$. $0.5 \mathrm{ml}$ of the aerobic cultures or anaerobic cultures were added into three $15 \mathrm{~mm} \times 18 \mathrm{~cm}$ glass tubes containing either $12 \mathrm{ml}$ saline (control), $12 \mathrm{ml}$ PAA solution or $12 \mathrm{ml} \mathrm{NaClO}$ solution, mixed with a whirlimixer for $20 \mathrm{~s}$ and leave them for $10 \mathrm{~min}$ at $23^{\circ} \mathrm{C}$. Subsequently, $0.5 \mathrm{ml}$ of each cell suspension was diluted into $4.5 \mathrm{ml}$ saline and mixed as described above in order to rapidly reduce the toxicity of oxidizing agents greatly (Grönholm et al., 1999). Subsequently, the surviving cells were enumerated by CFU.

\section{DETERMINATION OF CFU}

Cell suspensions were serially diluted in saline (0.9\%, pH 5.8) and transferred to MRS agar plates (Merck), then incubated at $30^{\circ} \mathrm{C}$ for 5 days, with analysis on day 3-5.

\section{MICROSCOPIC METHOD}

Peracetic acid was chosen as the oxidizing agent for microscopic analysis, but in order to reduce the number of killed cells, the concentration of PAA was decreased to $0.001 \%$. The treatment was otherwise the same as previously described.

Table 1 | Overview of the strains used in this study.

\begin{tabular}{llll}
\hline Abbreviation & Strain & origin & Type \\
\hline JK09 & $\begin{array}{l}\text { Lactobacillus brevis } \\
\text { JK09 }\end{array}$ & Danish craft beer & Wild type \\
JK09-horA & $\begin{array}{l}\text { Lactobacillus brevis } \\
\text { JK09-horA* }\end{array}$ & Danish craft beer & Plasmid cured \\
MI2158 & Lactobacillus brevis & DSM20054T & Wild type \\
& MI2158 & & \\
HF01 & Lactobacillus brevis & Danish craft beer & Wild type \\
& HF01 & & \\
HF02 & Lactobacillus brevis & Danish craft beer & Wild type \\
& HF02 & & \\
\hline
\end{tabular}

*This strain was cured from the horA plasmid.
The microscope set-up was the same as described by Ryssel et al. (2013). After the treatment with PAA, $5 \mu \mathrm{l}$ of the cell suspension was transferred to the bottom of a well in an Ibidi $\mu$-Slide 8 -well chamber (hydrophobic, uncoated, sterile, ibidi GmbH, München, Germany). The dead cell impermanent dye PI (Molecular Probes, Invitrogen, Oregon) was previously added to molten MRS agar to a final concentration of $2.0 \mu \mathrm{g} / \mathrm{ml}$ at $45^{\circ} \mathrm{C}$, and $300 \mu \mathrm{l}$ of the molten MRS-PI agar medium was added slowly to the well (to prevent the cells from leaving the bottom surface). The addition of MRS-PI agar constituted time zero for the experiment. After the agar solidified, the chamber was placed in the automated microscope stage, and a random spot was chosen in each well as the starting position. Subsequently, a total of 49 positions were recorded in a $7 \times 7$ grid, which was programmed into the software. The pre-programmed grid was used to avoid user bias when selecting appropriate spots in the specimen. Furthermore, the 49 positions enabled us to obtain more valid information about the heterogeneity of the specimen. The recording of all 49 positions were then repeated throughout the experiment. Brightfield images and PI fluorescent images were captured using MetaMorph 7.0 software package (Molecular Devices Inc., Silicon Valley, CA, USA). The chamber was left on the microscope stage at $23^{\circ} \mathrm{C}$ during the entire experiment.

\section{DATA ANALYSIS}

\section{Calculation of log reduction}

Log reduction was defined as the difference between the log CFU count of saline treatment (control) and disinfectant treatment.

\section{Calculation of survival in percentage}

In order to compare the microscopic analysis and the CFU, the survival percentage was calculated as follows.

$$
\text { Survival }_{\mathrm{CFU}}=\mathrm{CFU}_{\text {Treatment }} / \mathrm{CFU}_{\mathrm{Control}} \text { (Treatment }
$$
is control treatment or PAA treatment)

Survival $l_{\text {microscope }}=$ Num $_{\text {dividing cells }} / \mathrm{Num}_{\text {Total cells at time zero }}$

\section{Cell size analysis}

The brightfield images were analyzed with the free image analysis software image J [version 1.48; National Institutes of Health $(\mathrm{NIH})$, Bethesda, MD, USA $\left.{ }^{1}\right]$. Before cell division occurs, the size of individual cells is measured directly by pixels, but after cell division, the areas of the growing microcolonies were measured.

\section{Calculation of lag time and $\mu_{\max }$}

Growth data (time and cell size) were analyzed using the DMFit software available on the Combase website ${ }^{2}$. Growth data were fitted to the model proposed by Baranyi and Roberts (1994) for estimation of lag time ( $\lambda$, hour) and maximum specific growth rates ( $\mu_{\max }$, Ln pixels/hour) of each growth curve.

\section{RESULTS}

\section{EFFECT OF OXIDIZING AGENTS ON LAB}

Figure 1 shows the log reduction after treatment with PAA and $\mathrm{NaClO}$ on five Lactobacillus brevis strains. The initial log CFU of

\footnotetext{
${ }^{1}$ http://imagej.nih.gov/ij/

${ }^{2}$ http://www.combase.cc/index.php/en/
} 


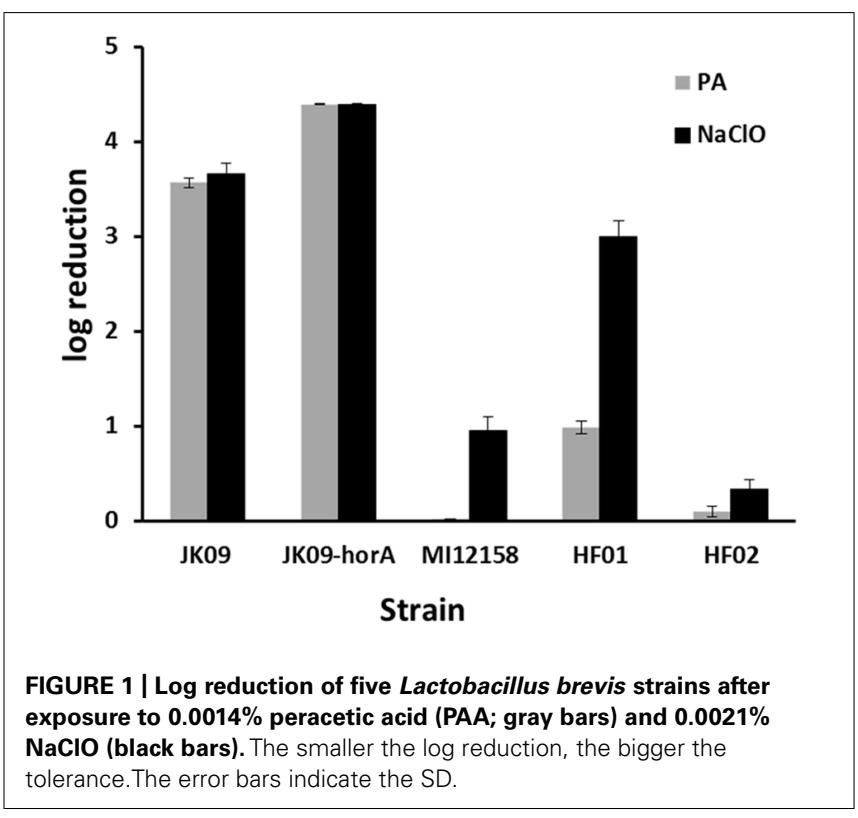

all the strains after anaerobic cultivation was $8.72 \pm 0.10$ (mean value $\pm \mathrm{SD}$ ). The two disinfectants exhibited different effectiveness against the different strains. After PAA treatment, JK09-hor A, which is a plasmid-cured strain, was the most sensitive strain, while the non-beer associated bacteria MI12158 was the most tolerant one; for $\mathrm{NaClO}$ treatment, JK09-hor A was still the most sensitive and HF02 was the most tolerant.

The two strains HF01 and JK09 were subsequently chosen for further experiments in a microscopic set-up, because they are both beer spoilage bacteria, and exhibited varying levels of sensitivity toward PAA, which was selected as the oxidizing agent for the microscopic analysis. The isolate HF02 was very tolerant toward the oxidizing agents, which would impede the microscopic analysis, and on the other hand, $\mathrm{NaClO}$ did not produce a pronounced difference between HF01 and JK09.

\section{GROWTH UNDER AEROBIC AND ANAEROBIC CULTIVATIONS}

Both HF01 and JK09 grew better under aerobic cultivation. For HF01, the aerobic culture reached $\mathrm{OD}_{600}$ of 1.5 after16h and the anaerobic culture after $21 \mathrm{~h}$. For JK09, the time was $14 \mathrm{~h}$ and $18 \mathrm{~h}$ under aerobic and anaerobic cultivations, respectively. In addition, the $\mathrm{pH}$ at $\mathrm{OD}_{600}$ of 1.5 under aerobic and anaerobic cultivations were 4.91 and 4.94 for HF01, whereas the $\mathrm{pH}$ of JK09 were 4.85 and 4.93 , respectively.

\section{COMPARISON BETWEEN CFU AND MICROCOLONY FORMATION}

For all treatments, colony forming units were detected up to 5 days by traditional CFU method, and microcolonies were observed for up to 2 days in the microscopic method. In the control experiments of HF01 and JK09, the size of the individual (macro) colonies on the plates was comparatively large and very similar and the number of colonies would not increase after 3 days of incubation. In contrast, the number of colonies increased for up to 5 days after exposure to PAA, and the size of colonies were heterogenous, since some colonies were as large as in the control, and other
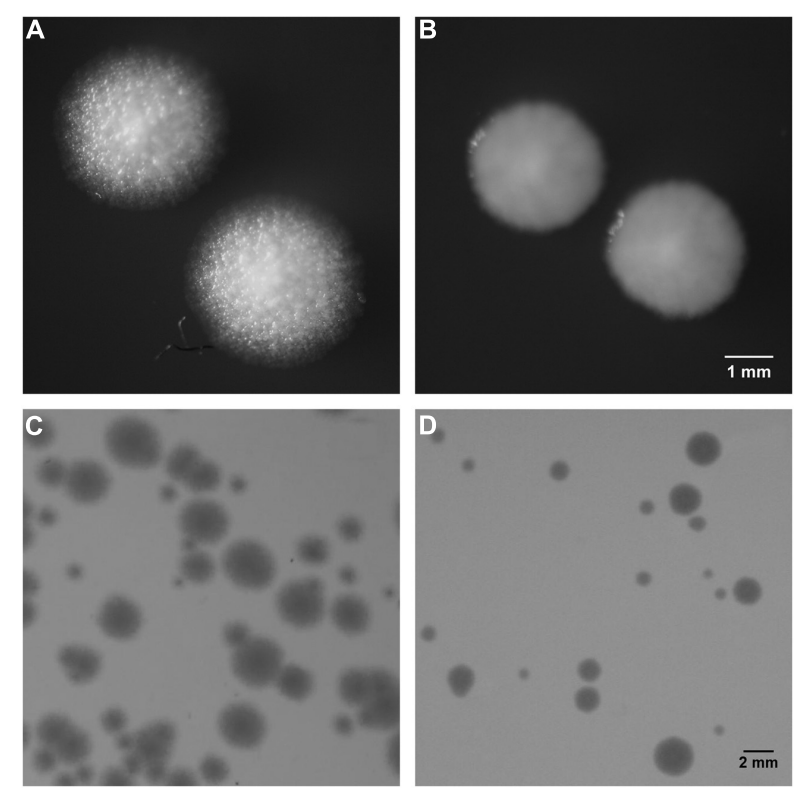

FIGURE 2 | Colony morphology of HF01 and JK09 on MRS agar after anaerobic cultivation. The colonies were photographed after 5 days of incubation. HF01 control (A), JK09 control (B), HF01 exposed to PAA (C), JK09 exposed to PAA (D). Strain HF01 grows as rough colonies while JK09 grows as smooth colonies. For both HF01 and JK09, the colonies without treatment were uniform in size, in contrast, the colonies after treatment with PAA were varying in size. (A) and (B) were illuminated from above to highlight the surface structure; (C) and (D) were illuminated from below to visualize the size difference of the colonies.

colonies were still much smaller on day 5. Figure 2 is an example of colony morphologies after anaerobic cultivation, the colony morphologies were similar after aerobic cultivation (results not shown).

A clear difference in colony morphology between HF01 and JK09 could be observed both on the plates (Figures 2A,B) and in the microscope (Figure 3). For HF01, the surface of (macro)colonies was rough and the edge appeared fluffy (Figure 2A). In the microscope, the microcolonies did not develop in all directions equally, but in a more random fashion and there were sometimes empty space within a microcolony (Figure 3E). For JK09, the surface of the (macro)colonies was smoother, and the edge was rounder (Figure 2B), with the growth of the microcolony expanding more equally in all directions (Figure $\mathbf{3 H}$ ).

Although we conventionally assume that all untreated cells (i.e., control) would grow and form colonies, it was observed that some individual cells never started to divide under the microscope (Figure 6), and therefore the calculation of the survival in the microscopic method will not reach $100 \%$.

Table 2 shows that there is good reproducibility of both methods, although the variation between repetitions of CFU could be up to $17.4 \%$, where the variation between repetitions of the microscopic method was up to $12.8 \%$. In general, for the control, the survival of the CFU method is slightly higher than that of the microscopic method, from $1.9 \%$ to $7.1 \%$. But in most cases, after PAA, the survival of the microscopic method is higher, from $-0.7 \%$ to $25.9 \%$. We can also 


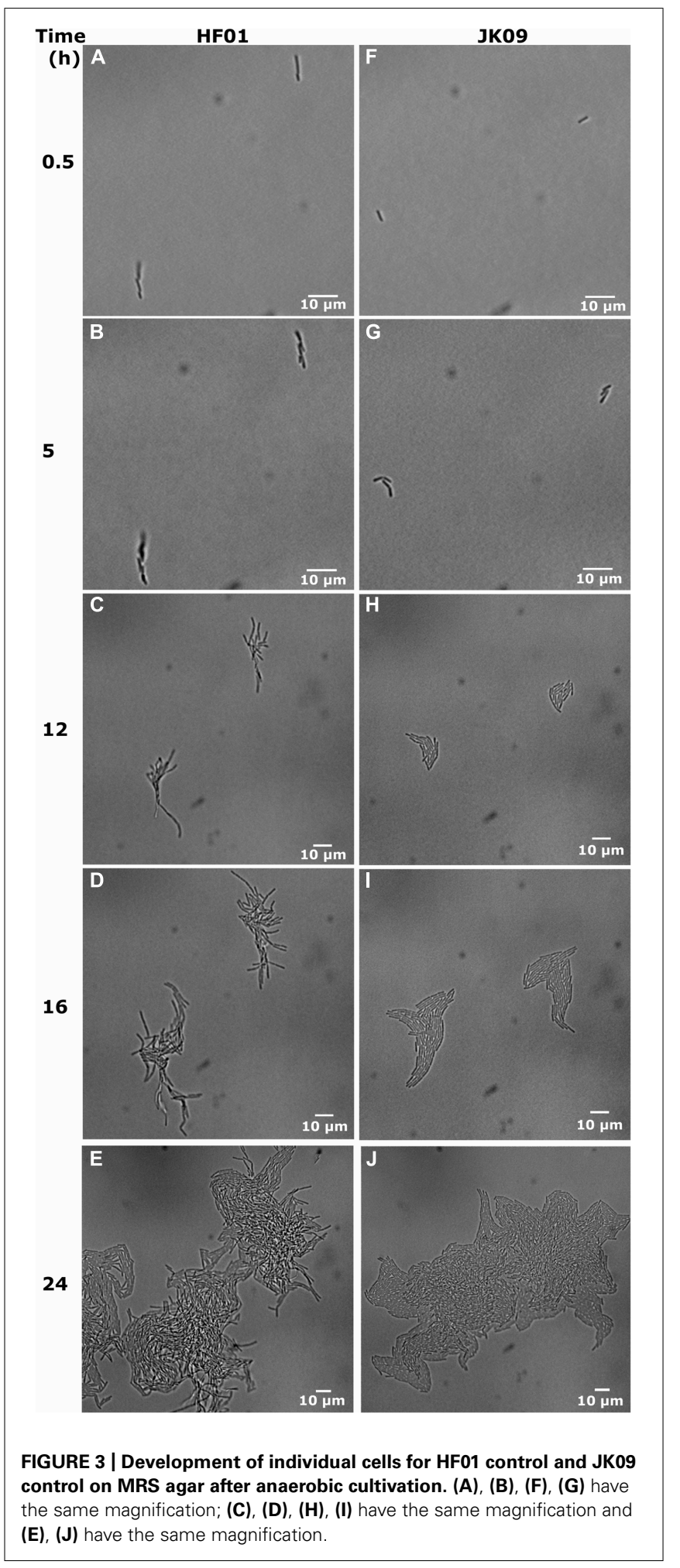

see from Table 2 that after exposure to PAA, the survival rate of HF01 was always higher than that of JK09 regardless of cultivation, and the survival rates of both HF01 and JK09 after aerobic cultivation were significantly higher than after anaerobic cultivation.
Table 2 | Comparison of survival rate measured by CFU and microscopic method.

\begin{tabular}{|c|c|c|c|c|c|}
\hline Strain & Cultivation & repetition & Treatment & $\begin{array}{l}\text { CFU } \\
\text { method \% }\end{array}$ & $\begin{array}{l}\text { Microscopic } \\
\text { method \% }\end{array}$ \\
\hline \multirow[t]{8}{*}{ HF01 } & Anaerobic & 1 & Control & 100.0 & 93.6 \\
\hline & & & PAA & 39.7 & 38.6 \\
\hline & & $2^{*}$ & Control & 100.0 & 92.9 \\
\hline & & & PAA & 47.4 & 51.4 \\
\hline & Aerobic & 1 & Control & 100.0 & 97.8 \\
\hline & & & PAA & 72.4 & 94.2 \\
\hline & & $2^{*}$ & Control & 100.0 & 97.1 \\
\hline & & & PAA & 85.7 & 96.5 \\
\hline \multirow[t]{8}{*}{ JK09 } & Anaerobic & 1 & Control & 100.0 & 97.5 \\
\hline & & & PAA & 1.9 & 1.3 \\
\hline & & $2^{*}$ & Control & 100.0 & 98.1 \\
\hline & & & PAA & 1.0 & 0.3 \\
\hline & Aerobic & 1 & Control & 100.0 & 97.8 \\
\hline & & & PAA & 32.1 & 58.0 \\
\hline & & $2^{*}$ & Control & 100.0 & 98.6 \\
\hline & & & PAA & 49.5 & 55.6 \\
\hline
\end{tabular}

* Experiments which were used to draw the growth curves of individual cells in Figure 5.

\section{MICROSCOPIC METHOD}

In Figure 3, we show the universal behavior of individual cells in the control experiments after anaerobic cultivation. Usually the cells elongate to two or three times the initial length, where after we observe the division into two or three cells. The cells continue to multiply, and eventually form a microcolony. We also observed a few cells that increased in cell length up to six times the initial length before division, while very few other cells prolonged a little but never started dividing (Figure 6).

Staining with PI did not by itself affect viability of Lactobacillus brevis (results not shown). Examples of corresponding brightfield and PI images of JK09 after anaerobic cultivation followed by PAA treatment are shown in Figure 4. At $44 \mathrm{~h}$, there are some red cells randomly distributed inside the microcolony (Figure 4B). But only two cells out of the initial seven non-growing cells were red. After $4 \mathrm{~h}$, the number of red cells increased within the extending microcolonies, and one additional individual cell turned red (Figure 4D).

The number of individual cells at time zero was from 0 to 14 in each image, and the total individual cell number in 49 images was between 200 and 500 . The growth curves of 50 dividing cells for each treatment are shown in Figures 5A-D (except for JK09 after anaerobic cultivation after PAA treatment, where only four cells divided). The experiments were repeated, and both strains showed good repeatability (results not shown). The experiment was stopped when the dividing microcolonies merged. It can be seen that for treatment with saline (control, blue lines), the growth of HF01 was similar to that of JK09 after the same cultivation, but with a certain variation in growth of individual cells. After 

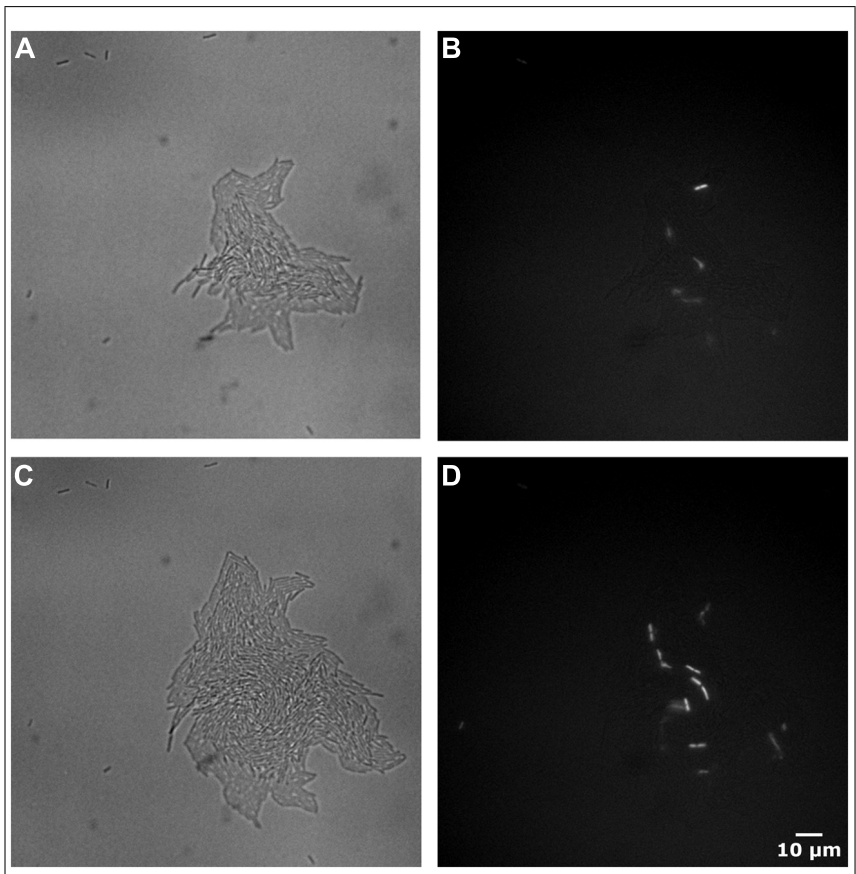

FIGURE 4 | Images of brightfield and PI fluorescent of JK09 after anaerobic cultivation followed by exposure to PAA. Brightfield at $44 \mathrm{~h}$ (A), propidium iodide (PI) fluorescent at $44 \mathrm{~h}$ (B), brightfield at $48 \mathrm{~h}$ (C), PI fluorescent at $48 \mathrm{~h}$ (D).

treatment with PAA (red lines), the individual growth curves were quite diverse for both strains.

The distributions of $\lambda$ and $\mu_{\max }$ values, estimated by the Baranyi and Roberts (1994) primary model for 340 microcolonies (14 microcolonies could not fit the model) originating from individual cells, are shown in Figures $\mathbf{5 a - d}$. After saline treatments (control, blue dots), the median $\lambda$ values of JK09 were a little bit smaller than that of HF01 regardless of cultivation. The median $\lambda$ values for both strains were also slightly smaller after aerobic conditions. The median $\mu_{\max }$ were almost similar for all control experiments. After PAA treatments (red dots), the median $\lambda$ values were distinctly bigger and the median $\mu_{\max }$ were clearly lower compared with the same strain and incubation in the control experiment. For HF01, the aerobic incubation gave smaller median $\lambda$ values and higher median $\mu_{\max }$ than the anaerobic incubation. It is impossible to draw conclusions about the influence of incubation on JK09, due to the limited number of dividing cells after anaerobic cultivation. We can also see that both $\lambda$ and $\mu_{\max }$ exhibit a significant variability for each experiment, especially after exposure to PAA. However, we could not find a clear correlation between $\lambda$ and $\mu_{\max }$. As an example, we show the calculated $\lambda$ and $\mu_{\max }$ from three neighbor colonies (Figure 6). The cells have almost similar distance to the other two cells, but with a distinct difference in $\lambda$ and $\mu_{\max }$.

\section{DISCUSSION}

As previously mentioned, there may be an inverse correlation between hop resistance and oxidation resistance. However, in this study a plasmid cured strain lacking the hop resistance gene horA had a slightly higher sensitivity toward oxidizing compounds compared to the wild type JK09 (Figure 1). This does not support the idea of an inverse correlation, but the plasmid cured strain also exhibited similar hop resistance to the wild type (results not shown), which suggests that hor A is neither important for hop resistance nor oxidation resistance.

It was previously found that Lactobacillus brevis ATCC 14869 exhibit Smooth(S)-type colonies when grown under anaerobic conditions, whereas the majority of colonies exhibit a Rough(R)type morphology under aerobic conditions (Jakava-Viljanen et al., 2002). In our study, the two Lactobacillus brevis strains HF01 and JK09 had distinctly different morphologies as JK09 exhibited S-type morphology and HF01 exhibited R-type morphology (Figure 2). However, their morphologies appeared to be less variable, as they retained the same morphology after PAA treatment, and the morphology was the same after aerobic and anaerobic cultivation. The study of Jakava-Viljanen et al. (2002) indicated that an oxidative environment promotes the formation of R-type colonies, which could suggest that strains with R-type morphology has an increased survival after exposure to oxidative compounds. This is consistent with our findings, where HF01 (R-phenotype) was more tolerant toward PAA.

In addition, our microscopic results suggest that already when microcolonies are formed, a distinct difference in colony morphology can be observed, which may predict the resulting morphology of macrocolonies (Figures 2A,B and 3).

It is clear that the microscopic method is more rapid than the CFU method for detection of dividing cells, (Asano et al., 2009). The traditional approach required at least 2 days before visible colonies (consisting of millions of cells) could be detected. In the present study, small colonies continued to appear until five days after the PAA treatment. The origin of these colonies is cells that can be considered 'hard-to-culture' (Suzuki, 2011), but our microscopic results suggests that this phenomenon can be attributed to the large variation in lag time of individual cells, after PAA treatment. This could be the reason why some survival rates using the CFU method were $20 \%$ lower than that using the microscopic method and with similar big differences between repetitions in the CFU method (Table 2). It is possible that some individual cells started division so late that the (macro)colonies were too small to be observed on the last day of the experiment. However, the microscopic method could observe cell elongation and division down to few hours after the beginning of the experiment. The microscopic method also has the potential to provide more details of the growth of individual cells into microcolonies. In our study, we can clearly see the growth dynamics of individual cells (Figure 3), where cells divided and eventually formed a microcolony. We also observed in our experiments that a few cells elongated, but then stopped dividing (Figure 6). This type of subpopulation cannot be observed with a CFU method, although the cells may possess some amount of biochemical activity. This might be another reason why the survival rates using the CFU method in some cases were $20 \%$ lower than the microscopic method.

Several studies have shown that individual cells exhibit heterogeneity in how they deal with stress in the same environment (Lianou et al., 2006; Métris et al., 2008; Muñoz-Cuevas et al., 2013). We observed that there are relatively small differences in the growth 

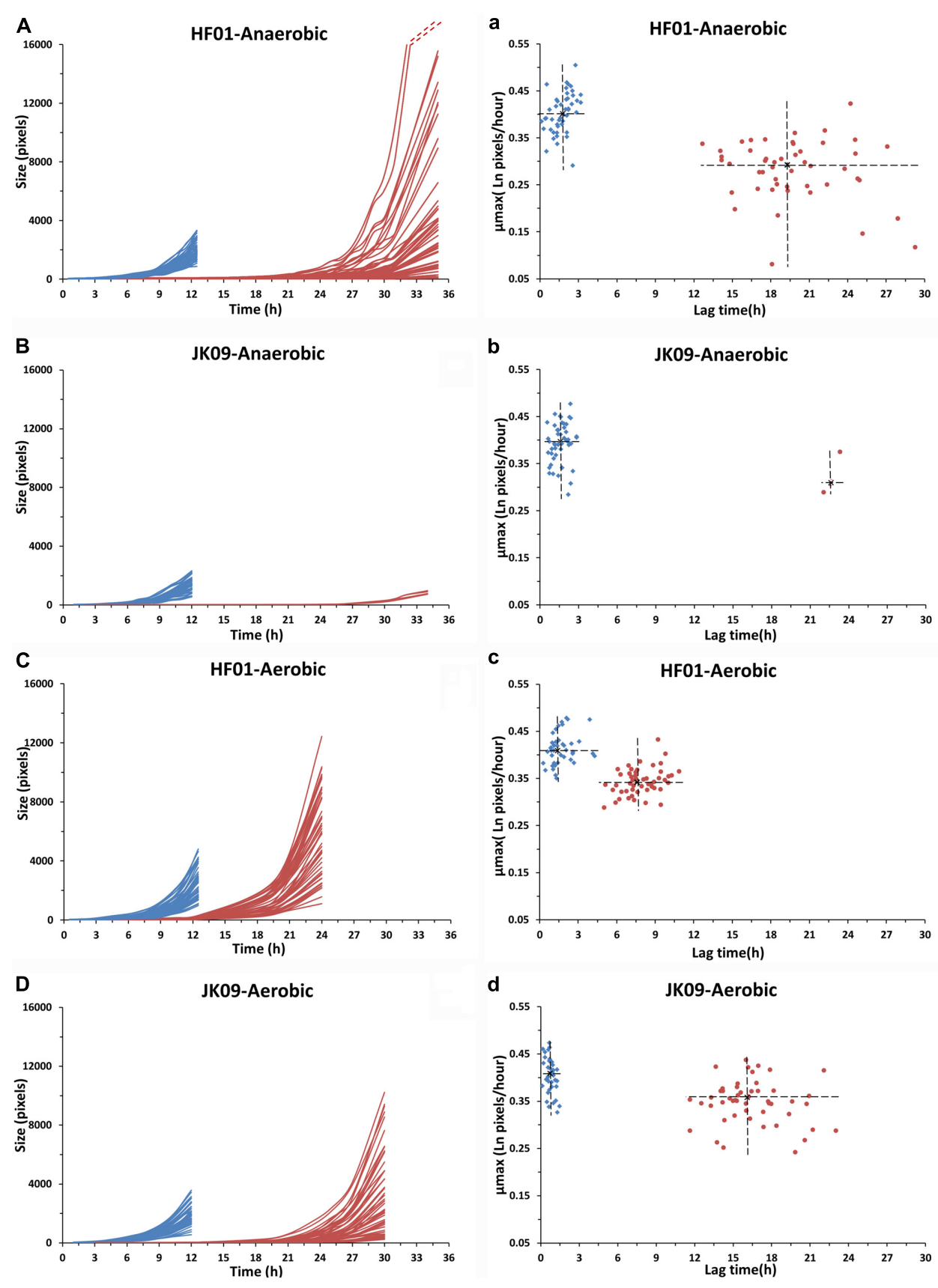

FIGURE 5 | Growth curves, lag times and $\mu$ max of individual cells of HF01 and JK09 after different treatments. Blue: after saline treatment (control); red: after PAA treatment. The dotted red lines indicate that the two fastest growing microcolonies reached approximately 31000 pixels at $35 \mathrm{~h}$. The center of the cross is the median of lag time and $\mu_{\max }(\mathbf{a}-\mathbf{d})$

curves and lag time of both strains in the control experiments (Figure 5), but extensive variation in growth behavior, survival, lag time and maximum growth rate was observed between the two strains after treatment with PAA (Table 2, Figure 5). This biological variability may be due to the genetic diversity between the strains, but the large variations in the resulting $\lambda$ and $\mu_{\max }$ of HF01 and JK09, especially after PAA treatments suggest that there is a phenotypic diversity that cannot be fully explained by presence of genes, as all of the individual cells of a strain can be considered clonal. After exposure to PAA, the lag time of the dividing cells of both strains increased pronouncedly. Interestingly, after the prolonged lag time, some cells of both strains exhibited the same $\mu_{\max }$ as in the control experiments, whereas other cells grow at a much slower rate. This is interesting, because it suggests that even after repair mechanisms have enabled the individual cells to divide, the resulting daughter cells in a microcolony continue to divide at a 

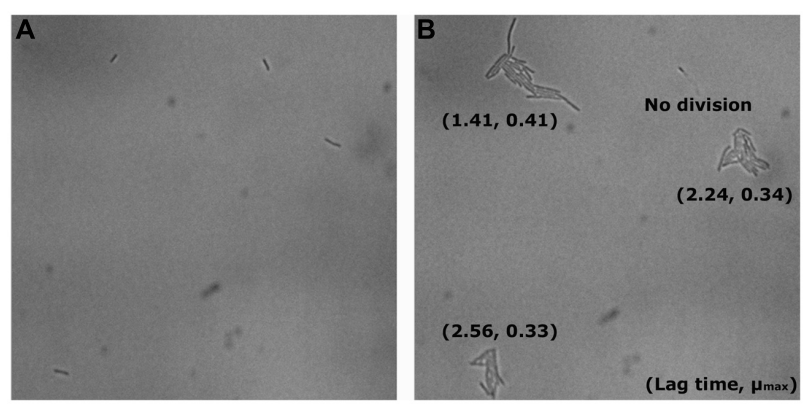

FIGURE 6 | Example of neighboring cells of JK09 in the control experiment after anaerobic incubation at $T=0$ (A) and $T=12 \mathrm{~h}$ (B).

rate that is predicted by the initial divisions, even though PAA has been removed. This result is inconsistent with the results from Kutalik et al. (2005), which points out that the extent of the lag phase only influence the first cell cycle and the subsequent division is uncorrelated to the cell history. In addition, we found that the lag time was significantly shorter and the $\mu$ max was distinctly higher after the aerobic cultivation for both strains (Figure 5). This result indicates that aerobic cultivation makes Lactobacillus brevis more tolerant to PAA. It may be because the bacteria have already built up some kind of defense mechanism or repair mechanism in order to protect them against oxygen during the aerobic cultivation. For example, high NADH oxidase activity and NADH peroxidase activity were found in Lactobacillus brevis after aerobic cultivation (Sakamoto and Komagata, 1996; Hummel and Riebel, 2003). In addition, there are marked differences in $\lambda$ and $\mu_{\max }$ of neighboring microcolonies (Figure 6). The differences are not caused by the proximity between the resulting microcolonies, because even after $12 \mathrm{~h}$ and several divisions, the resulting microcolonies still have similar distances to the neighboring colonies. It is likely that there would be an interaction between adjacent colonies when they are so close that they must share nutrients (propinquity effect), but it is unlikely that this is the case within the timeframe of our experiments.

The microscopic method used in this study can therefore provide quantitative data suitable for analysis of growth of individual cells. It should be noted that we only quantified the amount of pixels that are covered by microcolonies in two dimensions. When the microcolonies become very big (several hundred cells), the microcolony started to take on the traditional three-dimensional structure (Figures 4A,C), where a simple measure of area is no longer adequate. However, during many divisions, the cells are primarily growing on the surface of the agar, as our cells are enclosed between the coverslip that constitutes the bottom of the well and the agar. Additionally, if the microcolonies expand in less coordinated fashion such as HF01, where there can be visible holes inside the microcolony, it is important to subtract the area of the holes to obtain a valid estimate of cell growth.

Propidium iodide is a popular red-fluorescent DNA counterstain for estimating the amount of dead cells in a bacterial population (Bunthof etal., 2001; Rault et al., 2007). Due to its molecular weight and charge, it only penetrates cells with a damaged plasma membrane. As PI does not fluoresce without the presence of DNA, we incorporated PI into our growth matrix, in order to determine when and how, individual cells begin to die. Surprisingly, the large proportion of cells that failed to divide after treatment with PAA, did not exhibit red fluorescence (Figures 4B,D). In JK09, the survival after anaerobic cultivation after PAA treatment was around $1 \%$, so we would expect to observe 99\% red cells. This observation suggests that PAA does not compromise the membrane integrity of cells that fail to divide, and PI would therefore be a poor indicator of the efficacy of PAA. To rule out potential artifacts in our experiments, we determined that PAA treated cells that were afterward exposed to $70 \% \mathrm{EtOH}$ (which destroys the membrane) all stained red in our setup (results not shown). As microcolonies started to grow, we observed that a few individual cells turned red, which indicates that these cells have lost their membrane integrity (Figure 4). The cells appeared randomly within the microcolony, which does not suggest that they died from nutrition depletion or accumulation of toxic substances. If the cells should die from nutrient depletion or accumulation of toxic substances, it would be expected that the majority of the dead cells would be located in the center of the microcolony, this observation was previously reported by Ryssel et al. (2013).

In conclusion, the investigated beer spoilage LAB after different cultivations exhibit different sensitivity toward PAA, but there is no indication that the tolerance toward PAA is inversely correlated to the potential to spoil beer. On the other hand, the present study demonstrates a novel approach to investigate the formation of microcolonies as an indicator of physiological fitness. The method provides results faster than CFU determination, but seems to correlate very well with CFU. Furthermore, the method provides a tool to investigate the phenotypic heterogeneity of a clonal population, which can be expanded to many interesting aspects.

\section{ACKNOWLEDGMENT}

The authors are grateful to China Scholarship Council for financial support.

\section{REFERENCES}

Asano, S., Iijima, K., Suzuki, K., Motoyama, Y., Ogata, T., and Kitagawa, Y. (2009). Rapid detection and identification of beer-spoilage lactic acid bacteria by microcolony method. J. Biosci. Bioeng. 108, 124-129. doi: 10.1016/j.jbiosc.2009.02.016 Baranyi, J., and Roberts, T. A. (1994). A dynamic approach to predicting bacterial growth in food. Int. J. Food Microbiol. 23, 277-294. doi: 10.1016/01681605(94)90157-0

Behr, J., and Vogel, R. F. (2010). Mechanisms of hop inhibition include the transmembrane redox reaction. Appl. Environ. Microbiol. 76, 142-149. doi: 10.1128/aem.01693-09

Bunthof, C. J., Bloemen, K., Breeuwer, P., Rombouts, F. M., and Abee, T. (2001). Flow cytometric assessment of viability of lactic acid bacteria. Appl. Environ. Microbiol. 67, 2326-2335. doi: 10.1128/aem.67.5.2326-2335.2001

Elfwing, A., Lemarc, Y., Baranyi, J., and Ballagi, A. (2004). Observing growth and division of large numbers of individual bacteria by image analysis. Appl. Environ. Microbiol. 70, 675-678. doi: 10.1128/aem.70.2.675-678.2004

Grönholm, L., Wirtanen, G., Ahlgren, K., Nordström, K., and Sjöberg, A. M. (1999). Screening of antimicrobial activities of disinfectants and cleaning agents against foodborne spoilage microbes. Z. Lebensm. Forsch. A 208, 289-298. doi: 10.1007/s002170050419

Hollerová, I., and Kubizniaková, P. (2001). Monitoring Gram positive bacterial contamination in Czech breweries. J. Inst. Brew. 107, 355-358. doi: 10.1002/j.2050-0416.2001.tb00104.x

Hummel, W., and Riebel, B. (2003). Isolation and biochemical characterization of a new NADH oxidase from Lactobacillus brevis. Biotechnol. Lett. 25, 51-54. doi: 10.1023/A:1021730131633 
Jakava-Viljanen, M., Åvall-Jääskeläinen, S., Messner, P., Sleytr, U. B., and Palva, A. (2002). Isolation of three new surface layer protein genes (slp) from Lactobacillus brevis ATCC 14869 and characterization of the change in their expression under aerated and anaerobic conditions. J. Bacteriol. 184, 6786-6795. doi: 10.1128/jb.184.24.6786-6795.2002

Kitis, M. (2004). Disinfection of wastewater with peracetic acid: a review. Environ. Int. 30, 47-55. doi: 10.1016/S0160-4120(03)00147-8

Koutsoumanis, K. P., and Lianou, A. (2013). Stochasticity in colonial growth dynamics of individual bacterial cells. Appl. Environ. Microbiol. 79, 2294-2301. doi: 10.1128/aem.03629-12

Kutalik, Z., Razaz, M., Elfwing, A., Ballagi, A., and Baranyi, J. (2005). Stochastic modelling of individual cell growth using flow chamber microscopy images. Int. J. Food Microbiol. 105, 177-190. doi: 10.1016/j.ijfoodmicro.2005. 04.026

Lianou, A., Stopforth, J. D., Yoon, Y., Wiedmann, M., and Sofos, J. N. (2006). Growth and stress resistance variation in culture broth among Listeria monocytogenes strains of various serotypes and origins. J. Food Prot. 69, 2640-2647.

Menz, G., Andrighetto, C., Lombardi, A., Corich, V., Aldred, P., and Vriesekoop, F. (2010). Isolation, identification, and characterisation of beer-spoilage lactic acid bacteria from microbrewed beer from victoria, Australia. J. Inst. Brew. 116, 14-22. doi: 10.1002/j.2050-0416.2010.tb00393.x

Mertens, L., Van Derlinden, E., and Van Impe, J. F. (2012). A novel method for high-throughput data collection in predictive microbiology: optical density monitoring of colony growth as a function of time. Food Microbiol. 32, 196-201. doi: 10.1016/j.fm.2012.04.001

Métris, A., George, S. M., Mackey, B. M., and Baranyi, J. (2008). Modeling the variability of single-cell lag times for Listeria innocua populations after sublethal and lethal heat treatments. Appl. Environ. Microbiol. 74, 6949-6955. doi: 10.1128/aem.01237-08

Muñoz-Cuevas, M., Guevara, L., Aznar, A., Martínez, A., Periago, P. M., and Fernández, P. S. (2013). Characterisation of the resistance and the growth variability of Listeria monocytogenes after high hydrostatic pressure treatments. Food Control 29, 409-415. doi: 10.1016/j.foodcont.2012. 05.047

Niven, G. W., Fuks, T., Morton, J. S., Rua, S. A. C. G., and Mackey, B. M. (2006). A novel method for measuring lag times in division of individual bacterial cells using image analysis. J. Microbiol. Methods 65, 311-317. doi: 10.1016/j.mimet.2005.08.006

Rault, A., Béal, C., Ghorbal, S., Ogier, J.-C., and Bouix, M. (2007). Multiparametric flow cytometry allows rapid assessment and comparison of lactic acid bacteria viability after freezing and during frozen storage. Cryobiology 55, 35-43. doi: 10.1016/j.cryobiol.2007.04.005
Rossoni, E. M. M., and Gaylarde, C. C. (2000). Comparison of sodium hypochlorite and peracetic acid as sanitising agents for stainless steel food processing surfaces using epifluorescence microscopy. Int. J. Food Microbiol. 61, 81-85. doi: 10.1016/S0168-1605(00)00369-X

Ryssel, M., Duan, Z., and Siegumfeldt, H. (2013). In situ examination of cell growth and death of Lactococcus lactis. FEMS Microbiol. Lett. 343, 82-88. doi: 10.1111/1574-6968.12134

Sakamoto, M., and Komagata, K. (1996). Aerobic growth of and activities of NADH oxidase and NADH peroxidase in lactic acid bacteria. J. Ferment. Bioeng. 82, 210-216. doi: 10.1016/0922-338X(96)88810-6

Suzuki, K. (2011). 125th Anniversary review: microbiological instability of beer caused by spoilage bacteria. J. Inst. Brew. 117, 131-155. doi: 10.1002/j.20500416.2011.tb00454.x

Suzuki, K., Asano, S., Iijima, K., and Kitamoto, K. (2008a). Sake and beer spoilage lactic acid bacteria — a review. J. Inst. Brew. 114, 209-223. doi: 10.1002/j.20500416.2008.tb00331.x

Suzuki, K., Asano, S., Iijima, K., Kuriyama, H., and Kitagawa, Y. (2008b). Development of detection medium for hard-to-culture beer-spoilage lactic acid bacteria. J. Appl. Microbiol. 104, 1458-1470. doi: 10.1111/j.1365-2672.2007.03669.x

Suzuki, K., Iijima, K., Sakamoto, K., Sami, M., and Yamashita, H. (2006). A review of hop resistance in beer spoilage lactic acid bacteria. J. Inst. Brew. 112, 173-191. doi: 10.1002/j.2050-0416.2006.tb00247.x

Vaughan, A., O’Sullivan, T., and Van Sinderen, D. (2005). Enhancing the microbiological stability of malt and beer - a review. J. Inst. Brew. 111, 355-371. doi: 10.1002/j.2050-0416.2005.tb00221.x

Conflict of Interest Statement: The authors declare that the research was conducted in the absence of any commercial or financial relationships that could be construed as a potential conflict of interest.

Received: 29 August 2014; accepted: 31 October 2014; published online: 26 November 2014.

Citation: Zhao Y, Knøchel S and Siegumfeldt H (2014) In situ examination of Lactobacillus brevis after exposure to an oxidizing disinfectant. Front. Microbiol. 5:623. doi: 10.3389/fmicb.2014.00623

This article was submitted to Food Microbiology, a section of the journal Frontiers in Microbiology.

Copyright (c) 2014 Zhao, Knøchel and Siegumfeldt. This is an open-access article distributed under the terms of the Creative Commons Attribution License (CC BY). The use, distribution or reproduction in other forums is permitted, provided the original author(s) or licensor are credited and that the original publication in this journal is cited, in accordance with accepted academic practice. No use, distribution or reproduction is permitted which does not comply with these terms. 


\title{
Laser-induced speckle scatter patterns in Bacillus colonies
}

\author{
Huisung Kim ${ }^{1}$, Atul K. Singh ${ }^{2}$, Arun K. Bhunia ${ }^{2}$ and Euiwon Bae ${ }^{1 *}$ \\ ${ }^{1}$ Applied Optics Laboratory, School of Mechanical Engineering, Purdue University, West Lafayette, IN, USA \\ ${ }^{2}$ Molecular Food Microbiology Laboratory, Department of Food Science, Purdue University, West Lafayette, IN, USA
}

\section{Edited by:}

Sophie Jeanson, Institut National de la Recherche Agronomique - INRA, France

Reviewed by:

Folarin Anthony Oguntoyinbo, University of Lagos, Nigeria

Stella Maris Reginensi Rivera,

Universidad de la República Oriental

del Uruguay, Uruguay

*Correspondence:

Euiwon Bae, School of Mechanical

Engineering, Purdue University,

585 Purdue Mall, West Lafayette,

IN 47907, USA

e-mail: ebae@purdue.edu
Label-free bacterial colony phenotyping technology called BARDOT (Bacterial Rapid Detection using Optical scattering Technology) provided successful classification of several different bacteria at the genus, species, and serovar level. Recent experiments with colonies of Bacillus species provided strikingly different characteristics of elastic light scatter (ELS) patterns, which were comprised of random speckles compared to other bacteria, which are dominated by concentric rings and spokes. Since this laser-based optical sensor interrogates the whole volume of the colony, 3-D information of micro- and macro-structures are all encoded in the far-field scatter patterns. Here, we present a theoretical model explaining the underlying mechanism of the speckle formation by the colonies from Bacillus species. Except for Bacillus polymyxa, all Bacillus spp. produced random bright spots on the imaging plane, which presumably dependent on the cellular and molecular organization and content within the colony. Our scatter model-based analysis revealed that colony spread resulting in variable surface roughness can modify the wavefront of the scatter field. As the center diameter of the Bacillus spp. colony grew from 500 to $900 \mu \mathrm{m}$, average speckles area decreased two-fold and the number of small speckles increased seven-fold. In conclusion, as Bacillus colony grows, the average speckle size in the scatter pattern decreases and the number of smaller speckle increases due to the swarming growth characteristics of bacteria within the colony.

Keywords: bacterial colony, diffraction, speckle, phase modulation, Bacillus

\section{INTRODUCTION}

Bacterial colonies consist of millions of individual cells interacting with each other in complex ways (Shapiro, 1992; Wimpenny, 1992; Shimada et al., 1995; Ben-Jacob et al., 1998). It is important to understand the mechanism of colony formation and the resulting morphology as it is a key to understand organizations and interactions among bacterial cells within a colony. The colony morphology is highly influenced by the shape of individual cells, cell wall components (peptidoglycan, teichoic acid, lipopolysaccharide), the extracellular components and appendages (flagella, fimbriae, curli), and cellular response to the environmental cues including nutrient availability, oxygen and other gases, salt, acidity, alkalinity, temperature, etc. (Nagai et al., 1971; Granek and Magwene, 2010). Therefore, the colony morphology of different bacteria could be used as a foundation for differential characterization using an interrogating tool.

Historically, bacterial colony pattern formation has drawn significant interest among diverse research fields. Microbiologists were more interested in determining how bacterial response to the environmental factors such as nutrient and agar hardness affect colony formation, while the physicist were aiming to recreate the colony formation scenario by using theoretical models. Shapiro and Wimpenny first observed bacterial colony formation in time and space (Shapiro, 1992; Wimpenny, 1992) and since then, many other researchers employed both experimental (Shimada et al., 1995; Ben-Jacob et al., 1998; Bees et al., 2000; Stecchini et al., 2001; Kaito and Sekimizu, 2007; Pipe and Grimson, 2008) and theoretical models (Kawasaki et al., 1997; Cohen et al., 1999; Kozlovsky et al., 1999; Lega and Passot, 2003) to explain such phenomenon. Among the various organisms tested, a majority of the study used Bacillus spp. as a model due to their swarming growth characteristics. Swarming colonies can generate diverse spatio-temporal patterns due to their reproduction and spreading mechanisms (Harshey, 2003). Therefore, we also studied Bacillus as a model organism to correlate the swarming colony morphology to the optical light scattering and speckle effect.

Since the first reports (Bae et al., 2007; Banada et al., 2007), optical light scattering of colony have expanded to differentiate diverse bacterial genera. The remarkable resolving power of the BARDOT originates from the accumulation and amplification of both microscopic structural and biochemical differences that exist among different bacterial colonies through an interrogating laser beam. When the laser beam passes through the bacterial colony, both 3D morphological and optical characteristics are integrated into 2D outgoing wave and encoding it on the coherent optical wavefront. The wavefront then propagates through near- and far-fields governed by the diffraction integral to form distinctive forward scattering pattern which serves as an optical "fingerprint."

Elastic light scatter (ELS) has been used by our group for bacterial colony differentiation and identification (Bae et al., 2007, 2011; Banada et al., 2009; Huff et al., 2012; Singh et al., 2014). Recently we have used this technology for differentiating Bacillus cereus (an important food poisoning species) and compared 
its colony scattering pattern with another ubiquitous species, Escherichia coli (Singh et al., submitted). Bacillus is highly motile and considered a swarming bacterium, which shows greater mobility on solid agar. Among the Bacillus spp., surprisingly, only B. polymyxa expressed typical ELS patterns of concentric rings and spokes consistent with other bacterial genera: Listeria, Salmonella, Vibrio, Escherichia, and Staphylococcus (Bae et al., 2007; Banada et al., 2009; Huff et al., 2012; Singh et al., 2014). While all other Bacillus species tested showed random speckles overlaid with some circular ring patterns (Singh et al., submitted). The scattering patterns of B. subtilis and B. polymyxa have shown significant differentiating characteristics. In this paper, we investigated the different optical properties of these two Bacillus species; (i) to understand how B. subtilis and B. polymyxa construct their colony using recently developed Integrated Colony Morphology Analyzer (ICMA) (Kim et al., 2013); (ii) to compare the theoretical prediction of ELS patterns with experimental data; and (iii) to calculate the speckle statistics to quantitatively correlate the optical phase modulation into the structure of the bacterial colony.

\section{MATERIALS AND METHODS SAMPLE PREPARATION}

Bacillus subtilis ATCC 6633 (B. subtilis), Bacillus polymyxa B719W (B. polymyxa), Bacillus cereus ATCC 14579 (B. cereus), and Bacillus thuringiensis DUP6044 (B. thuringiensis) cultures were used in this study. Cultures were inoculated in brain heart infusion (BHI) broth (Difco, MD, USA) and incubated at $37^{\circ} \mathrm{C}$ for overnight $(16 \mathrm{~h}$ ) with $130 \mathrm{rpm}$ shaker, 10 -fold serially diluted in $0.2 \mathrm{mM}$ phosphate buffered saline, $\mathrm{pH} 7.0$ (PBS) and diluents were plated on BHI and phenol red mannitol (PRM) agar plates (Becton Dickinson, NJ, USA) to obtain 50-100 colonies. Optical scatter patterns of the colonies were captured using the BARDOT instrument at 6-8 $\mathrm{h}$ that corresponds to colony diameter of about 550,750 , and $900 \mu \mathrm{m}$, respectively.

\section{FORWARD SCATTEROMETER}

Scatterometer (Figure 1A), consists of laser diode (LD) with $635 \mathrm{~nm}$ wavelength that was installed as a light source, and the light source directly illuminates a single bacterial colony grown on semi-solid agar plate. The diffracted light is captured using detector (CMOS camera, PL-B741, ON, Canada) with $1280(\mathrm{H}) \times$ $1024(\mathrm{~V})$ pixels, $6.7 \times 6.7 \mu \mathrm{m}$ pixel pitch which is located after the semi-solid agar plate with distance z. Motorized 2 axis lateral stage was integrated to the system, and the semi-solid agar plate was put on the 2 axis lateral stage for a full automatic measurement of optical scatter pattern. The Bacillus colonies were then picked from the plate, grown briefly $(4 \mathrm{~h})$ in $\mathrm{BHI}$ broth at $37^{\circ} \mathrm{C}$ and analyzed by multiplex PCR (mPCR) assay.

\section{DNA EXTRACTION AND mPCR}

Total DNA was extracted by boiling cultures as described earlier (Ngamwongsatit et al., 2008). mPCR was performed using gyrB gene-specific primers (BcF: 5' GTTTCTGGTGGT TTACATGG3'; BcR: 5'TTTTGAGCGATTTAAATGC $3^{\prime}$ ) and cry gene-specific primers (K5F: 5'AGG ACCAGGATTTACAGGAGG 3'; K3R: 5' GCTGTGACACGAAGGATATAGCCAC 3') (Kuo and Chak, 1996;

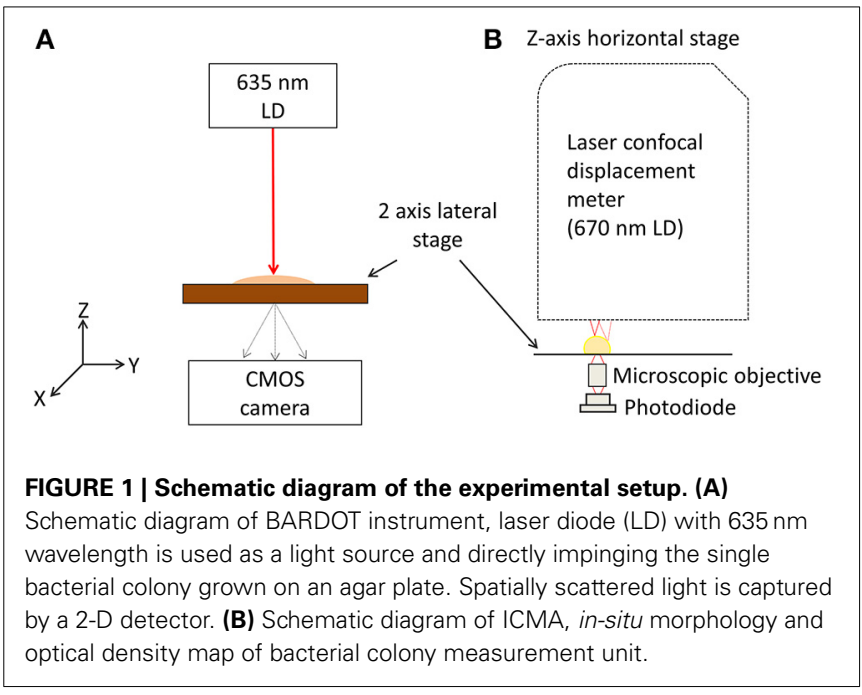

Manzano et al., 2003). The mPCR reaction mixture contained $200 \mu \mathrm{M}$ of each dNTP, $2.5 \mathrm{mM}$ of $\mathrm{MgCl}_{2}, 0.8 \mathrm{X}$ GoTaq Flexi buffer, $1 \mathrm{U}$ of GoTaq Flexi DNA polymerase (Promega), $0.2-0.3 \mu \mathrm{M}$ of primers, $60-90 \mathrm{ng}$ of template DNA and the reaction condition was optimized (Henegariu et al., 1997; Lorenz, 2012). Ultra-pure sterile water was used as negative control. PCR reaction was only considered valid when control reaction was either positive or negative as appropriate and $16 \mathrm{~S}$ rRNA gene was positively amplified with all DNA templates. All amplicons were analyzed on 1.2\% agarose gel.

\section{INTEGRATED COLONY MORPHOLOGY ANALYZER (ICMA)}

As shown in Figure 1B, the laser confocal displacement meter (CDM) (Keyence, LT9010M) is located at the top and attached to a linear horizontal translation stage (Edmund Optics, NJ, USA) in order to position the probing laser beam $(\lambda=670 \mathrm{~nm})$ along the vertical $\mathrm{z}$ axis and to focus it on the surface of colony. A motorized 2D lateral stage translated the Petri dish in the $x-y$ plane to align the diagnostic laser beam with each selected bacterial colony to obtain their 3-D profiles. Two linear motors (850GHS) connected to the ESP 300 multi-axis closed-loop controller (Newport, NY, USA) with the specification of a $42 \mathrm{~mm}$ maximum stroke and a $0.1 \mathrm{~mm}$ minimum step size control the movement of the $x-y$ stage. This instrument implements a high accuracy surface scanning method by using a laser light source with a Gaussian beam spot of approximately $2 \mu \mathrm{m}$. The position of the doublet lenses is controlled by a tuning fork moving up and down at high frequency. When the beam was focused on the sample (colony) surface, the reflected light traveled back along the original optical path and was directed toward the pinhole to produce the highest level of intensity on the light receiving element. If the laser is unfocused, some of the reflected light would be occluded at the pinhole. Therefore, the relative height of the measured surface was registered by the sensor positioned at the bottom of the tuning fork, and the values are calculated and converted to an absolute height value (Keyence-Corporation, 2006).

A micro-objective lens (Bausch \& Lomb, 20x, N.A. 0.4) is placed just below the Petri dish to focus the transmitted light 
onto the active area of the photodiode (Hamamatsu, S1087) (Figure 1B). This photodiode offered a low background noise (10 pA) with a wide dynamic range and provided a spectral response from 320 to $730 \mathrm{~nm}$ with the peak sensitivity at $560 \mathrm{~nm}$. The active area for this photodiode is $1.3 \times 1.3 \mathrm{~mm}^{2}$, providing a photo sensitivity of $0.19 \mathrm{~A} / \mathrm{W}$ for the incoming laser from the CDM sensor head on which a custom built active components driven I-V converter and preamp were installed at a preamp section. A custom built micro controller unit (MCU) (AVR128, Atmel) was used as a data acquisition unit. Through use of the MCU's internal 10 bit A/D conversion, all signals from the photodiode circuit and CDM were captured and transferred simultaneously to the PC by means of serial communication. All sequences were controlled by a custom built graphic user interface (GUI) at the PC, which was developed using Microsoft Visual Studio 2008 and both were analyzed and visualized in MATLAB.

\section{MODELING SPECKLE EFFECT FROM ELASTIC LIGHT SCATTERING}

The bacterial colony and a semi-solid media are positioned at the aperture plane, and the forward scattering pattern is captured at the image plane, defined as $\left(\mathrm{x}_{\mathrm{a}}, \mathrm{y}_{\mathrm{a}}\right)$ and $\left(\mathrm{x}_{\mathrm{i}}, \mathrm{y}_{\mathrm{i}}\right)$, respectively. A bacterial colony is modeled as a bell curve shape with tailing edge (Gaussian-like profile) where colony center height and radius is defined as $H_{0}$ and $r_{c}$, respectively. Based on Rayleigh and Sommerfeld formation of diffraction, and Fresnel diffraction approximation (Bae et al., 2007, 2010), intensity of electric field at aperture and image plane is derived as Equations (1) and (2), respectively, where distance between aperture plane to a point at image plane, $r_{a i}$ is assumed as Equation (3).

$$
\begin{aligned}
E_{a}\left(x_{a}, y_{a}, z\right)= & E_{0} \exp \left[-\frac{\left(x_{a}^{2}+y_{a}^{2}\right)}{\omega^{2}(z)}\right] \exp (i k z) \\
& \exp \left[i k \frac{\left(x_{a}^{2}+y_{a}^{2}\right)}{2 R(z)}\right] \\
E_{i}\left(x_{i}, y_{i}\right)= & \frac{1}{i \lambda} \iint t\left(x_{a}, y_{a}\right) E_{a}\left(x_{a}, y_{a}\right) \exp \left[i k \Phi\left(x_{a}, y_{a}\right)\right] \\
& \frac{\exp \left[i k r_{a i}\right]}{r_{a i}} \cos \theta \mathrm{d} x_{a} \mathrm{~d} y_{a} \\
r_{a i}= & {\left[z_{i}^{2}+\left(x_{a}-x_{i}\right)^{2}+\left(y_{a}-y_{i}\right)^{2}\right]^{\frac{1}{2}} } \\
\cong & z_{i}\left[1+\frac{1}{2}\left(\frac{x_{a}-x_{i}}{z_{i}}\right)^{2}+\frac{1}{2}\left(\frac{y_{a}-y_{i}}{z_{i}}\right)^{2}\right]
\end{aligned}
$$

Since previous researchers focused on structural morphology of bacterial colony such as elevation and colony diameter, the colony was modeled as smooth curve with Gaussian profile function without considering surface roughness. In reality, a cross section of bacterial colony showed the accumulation of densely packed multiple layers of bacterial cells (Banada et al., 2009; Bae et al., 2010; Suchwalko et al., 2013; Marcoux et al., 2014). Furthermore, Bacillus colonies also showed some degree of surface roughness (Figures 2, 3). To simplify the modeling, surface roughness is generated by random signal with colony area, and superposed to
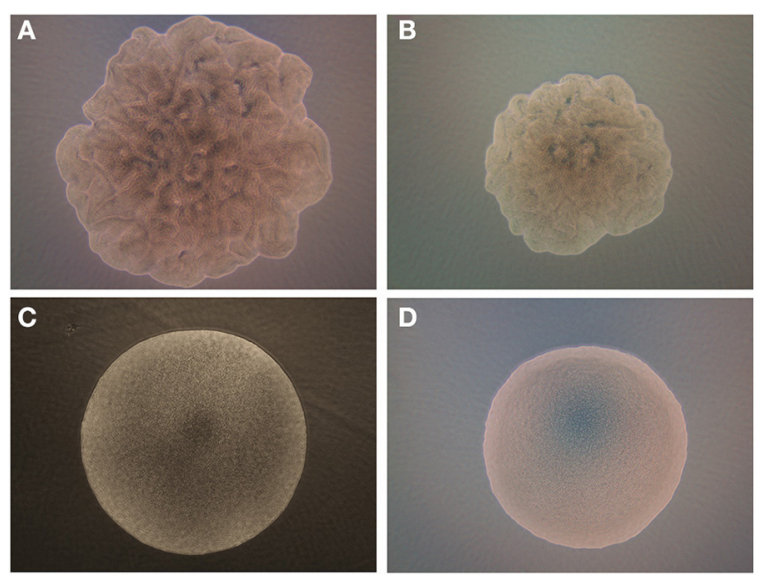

FIGURE 2 | Phase contrast images of the $B$. subtilis on (A) $\mathrm{BHI}$ and (B) PRM where colony shows swarming growth characteristics. For comparison, B. polymyxa on (C) BHI and (D) PRM are shown where colony forms a circular shape.

the colony morphology. Considering the surface roughness of the colony, intensity of electric field at image plane is simplified as Equation (4).

$$
\begin{aligned}
E_{i}\left(x_{i}, y_{i}\right)= & C \iint \mathrm{T}\left(x_{a}, y_{a}\right) \exp \left[i \Phi_{r}\right] \exp \left[i \Phi_{c}\right] \exp \left[i\left(\Phi_{g}+\Phi_{s}\right)\right] \\
& \exp \left[-2 \pi i\left(f_{x} x_{a}+f_{y} y_{a}\right)\right] \mathrm{d} x_{a} \mathrm{~d} y_{a}
\end{aligned}
$$

where $\mathrm{T}$ is amplitude modulator; $f_{x}$ and $f_{y}$ is defined as $x i /\left(\lambda z_{2}\right)$ and $y i /\left(\lambda z_{2}\right)$, known as a spatial Fourier frequency; $\Phi_{r}, \Phi_{q}, \Phi_{g}$, and $\Phi_{s}$ is radial, quadratic, Gaussian, and surface roughness phase component respectively, and defined as:

$$
\begin{aligned}
& \Phi_{r}\left(x_{a}, y_{a}\right)=\frac{k\left(x_{a}^{2}+y_{a}^{2}\right)}{2 R} \\
& \Phi_{q}\left(x_{a}, y_{a}\right)=\frac{k\left(x_{a}^{2}+y_{a}^{2}\right)}{2 z_{i}} \\
& \Phi_{g}\left(x_{a}, y_{a}\right)=k\left(n_{b a c}-1\right) H_{0} \exp \left[-\frac{\left(x_{a}^{2}+y_{a}^{2}\right)}{r_{c}^{2}}\right] \\
& \Phi_{s}\left(x_{a}, y_{a}\right)=k\left(\operatorname{random}\left(x_{a}, y_{a}\right)\right) \text { where }\left(x_{a}^{2}+y_{a}^{2}<r_{c}^{2}\right)
\end{aligned}
$$

The summation of four phase components, $\Phi_{\text {overall }}$ is working as phase modulator for the propagating light. The amplitude modulator $\mathrm{T}$ is derived as:

$$
\begin{aligned}
T\left(x_{a}, y_{a}\right)= & \exp \left[-\frac{\left(x_{a}^{2}+y_{a}^{2}\right)}{\omega^{2}(z)}\right] \frac{E_{\text {out }}}{E_{0}} \\
= & \exp \left[-\frac{\left(x_{a}^{2}+y_{a}^{2}\right)}{\omega^{2}(z)}\right]\left(1-r_{\text {air-bac }}\right)\left(1-r_{k}\right)^{2 l} \\
& \left(1-r_{\text {bac-agar }}\right) \\
\Phi_{\text {overall }}= & \Phi_{r}+\Phi_{q}+\left(\Phi_{g}+\Phi_{s}\right)
\end{aligned}
$$




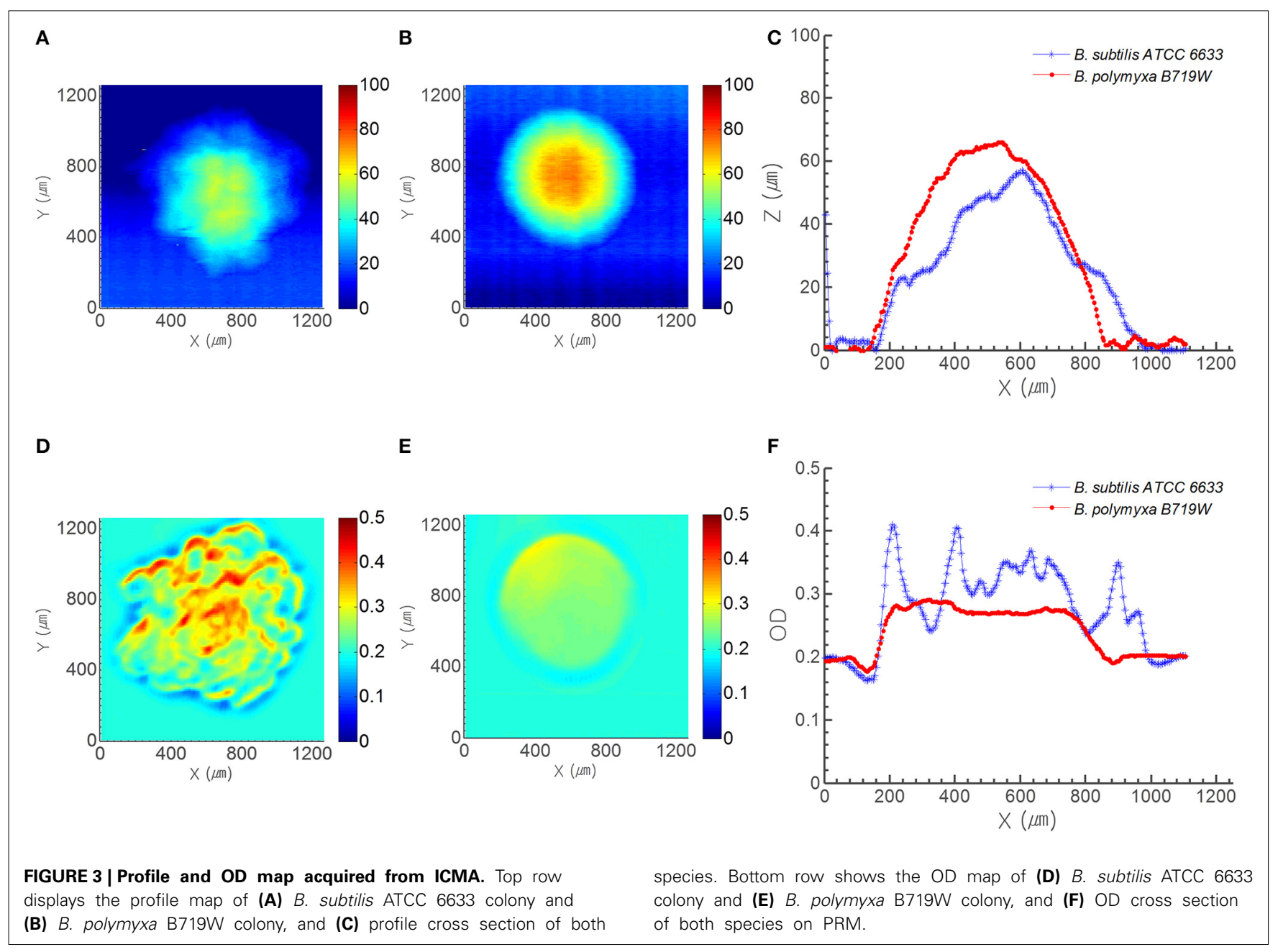

Coefficient of Equation (4), $\mathrm{C}$ is derived as:

$$
C=\frac{E_{0} \exp \left(i k n_{\text {agar }} \Delta_{\text {agar }}\right) \exp \left(i k H_{0}\right) \exp \left[i k\left(z+z_{i}\right)\right]}{\exp \left[i k\left(x_{i}^{2}+y_{i}^{2}\right) / 2 z_{i}\right]}
$$

where $\Delta_{\text {agar }}$ and $n_{\text {agar }}$ is defined as thickness of agar and refractive index of agar respectively.

\section{SPECKLE ANALYSIS}

To provide quantitative speckle analysis, a MATLAB code was constructed utilizing an image processing toolbox V2.6 (Vliet, 2014). A radial Gaussian blur was applied to the raw image to apply a low pass filter that eliminates the objects while keeps the slow change in the background, and the blurred image was subtracted from the raw image to remove the background noise. The subtracted image was binarized with locally adaptive threshold using approximation of gradient of background intensity to eliminate the external lighting and local CMOS sensor sensitivity characteristics effect on the image. Then, each speckle was segmented, grouped, and labeled. Number of pixels (size of the speckle) and feret diameter (Merkus, 2009) of each segmented group was computed, and analyzed.

\section{RESULTS}

Here, we report how colony morphology correlates with its speckle patterns using both theoretical and experimental approaches. Thus, each colony was first interrogated by ICMA and phase contrast microscopy to record height, transmittance and morphology.

\section{COLONY DEVELOPMENT PROFILES}

The phase contrast microscopic (PCM) images of colonies of $B$. subtilis and B. polymyxa on both BHI and PRM were contrasting (Figure 2). B. subtilis colony has an irregular boundary with bumpy and uneven surface structure on both media (Figures 2A,B). Due to the limitation of phase contrast microscopic setup, the 3-D elevation cannot be interpreted with current image but B. subtilis shows a representative swarming colony characteristics, where spatially localized variations of bacterial cell densities are observed. Meanwhile, B. polymyxa had symmetrical circular boundary with relatively smooth surface curvature (Figures 2C,D). As the PCM result showed, the colony forming characteristics of B. polymyxa was drastically different from the majority of other Bacillus species including B. subtilis, B. cereus, and B. thuringiensis. 


\section{COMPARISON OF TRANSMITTANCE AND ELEVATION PROFILE}

To compensate the phase contrast microscope image, ICAM was utilized to acquire spatially resolved optical characteristics from a single colony. Figures 3A,B show the morphology map of B. subtilis and B. polymyxa colony on PRM, respectively from morphology channel of ICMA; whereas panel (d) and (e) show their corresponding spatially resolved transmittance in optical density (OD) units with $670 \mathrm{~nm}$ light source. Colonies of both species on PRM agar were captured after $7 \mathrm{~h}$ of growth, when the center diameter of $B$. polymyxa colony reached to approximately $800 \mu \mathrm{m}$. Similar to the PCM result, morphology map by ICMA revealed that boundary of $B$. subtilis colony was irregular, while, B. polymyxa colony had near circular symmetrical boundary (Figures 3A,B). Comparison of morphology cross section for the both species at their center area showed that B. subtilis colony had random bumpy profile with rough surface, while $B$. polymyxa colony had bell curve-like profile with relatively smooth surface (Figure 3C). B. subtilis colony had approximate $890 \mu \mathrm{m}$ cross sectional diameter and $57 \mu \mathrm{m}$ of center peak height, while, B. polymyxa colony had $814 \mu \mathrm{m}$ diameter and $66 \mu \mathrm{m}$ of colony center height. Considering the colony aspect ratio (colony center height to diameter ratio) of each species, B. subtilis $(1: 15.6)$ colony had more flat cross sectional profile than B. polymyxa (1:12.4) at the same diameter. Furthermore, the 2-D transmittance map (cross-sectional OD profile) of both species shows more distinctive characteristics, which can be attributed to colony morphology and opacity (Figures 3C,F). Since the OD of BHI agar plate alone at $670 \mathrm{~nm}$ was approximately 0.2 , cross section of the OD result had 0.2 offset. Cross section of $B$. subtilis colony OD fluctuates from 0.24 to 0.41 , while $B$. polymyxa colony had OD with maxima 0.29 . Assuming that each species of bacterial cell had identical optical characteristic, this irregularity and randomness of OD can be ascribed to different spatial cell density that is translated into variation in light absorption. In addition, surface roughness of the $B$. subtilis colony can be modeled as variation in reflected and transmitted light intensity (Figure 3).

\section{ELS MEASUREMENT}

In previous studies, optical forward scattering pattern or ELS of Salmonella, E. coli, and Staphylococcus showed characteristics symmetric or concentric circle with some radial spokes (Banada et al., 2009). However, forward scattering pattern of Bacillus species (except B. polymyxa) showed speckle pattern (Figures 4A, 5). Diffraction pattern of $B$. subtilis colony is consisted of only small sized random speckle, while, $B$. polymyxa shows concentric circular pattern (Figure 4). To verify the time resolved speckle development of Bacillus species on their forward scattering pattern, B. subtilis, B. cereus, and B. thuringiensis were selected and their diffraction patterns were measured after $6-8 \mathrm{~h}$ of growth using BARDOT (Figure 5). As the incubation time increased from 6 to $8 \mathrm{~h}$, the diffraction patterns also evolved to fully developed speckles, i.e., structured patterns progressively became unstructured random speckles. All three Bacillus species displayed similar trend as the incubation time increased where average speckle numbers decreased.
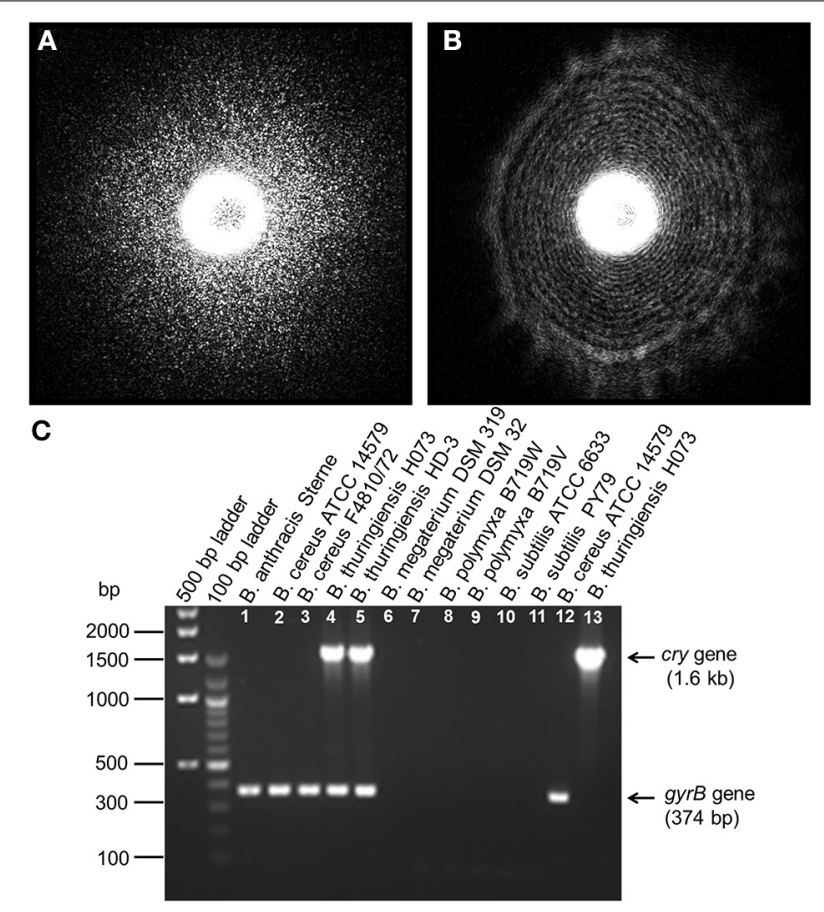

FIGURE 4 | Elastic light scatter (ELS) patterns on PRM agar for (A) B. subtilis ATCC 6633, and (B) B. polymyxa B719W; (C) Multiplex PCR (mPCR) for differentiation of Bacillus cereus-group strains (Lane 1-5) from other Bacillus species (Lane 6-11). mPCR resulted a 374 bp PCR product for all Bacillus cereus-group strains and a $1.6 \mathrm{~kb}$ size PCR product only for $B$. thuringiensis. Lane 12 and 13 represents single gene specific amplification for gyraseB gene in Bacillus cereus and cry gene in Bacillus thuringiensis. mPCR was performed with gyrase $B$ gene-specific and cry protein gene-specific primers.

\section{COMPARATIVE ANALYSIS OF EXPERIMENT WITH SIMULATION}

To investigate the effect of surface roughness on forward scattering pattern, other components of models are kept constant for Equations (5)-(11). The effect of random surface phase component $\left(\Phi_{s}\right)$ on forward scattering pattern was determined (Figure 6). Figure 6A shows the forward scattering pattern prediction when the surface phase component was equal to zero which means, the colony surface was devoid of any random structures. The predicted scatter image contained concentric ring patterns with small bright ring at the center and the thickness of rings increased as they moved outwardly. To mimic the random surface roughness effect, Equation (8) was adopted which is a random function multiplied by the wave number $k$, and is only influenced by bacterial colony region. The maximum amplitude of the random function for Equation (8) was chosen as $1 / 150,1 / 100$, and $1 / 80$ of colony center height for fine, medium, and coarse surface roughness, respectively. The surface roughness component $\left(\Phi_{s}\right)$ is added to Gaussian phase component $\left(\Phi_{g}\right)$, and worked as a part of phase modulator, which can deform the incident wave front. Predicted forward scatter patterns are shown for fine (Figure 6B), medium (Figure 6C), and coarse surface roughness (Figure 6D). For a diffraction pattern prediction with fine surface roughness effect, relatively larger size speckles and less 


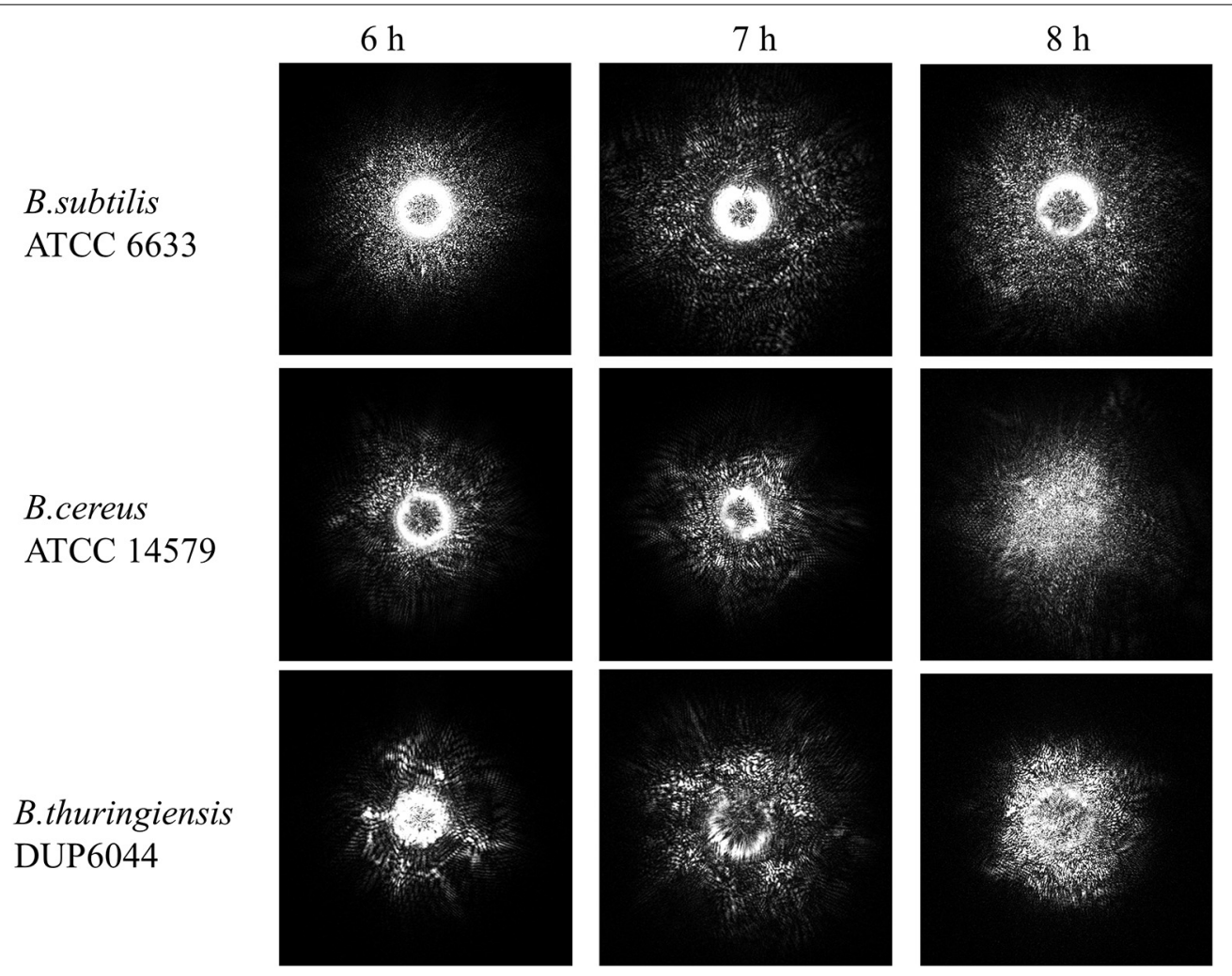

FIGURE 5 | Time-resolve ELS patterns for three different Bacillus species: $\boldsymbol{B}$. subtilis, $\boldsymbol{B}$. cereus, and $\boldsymbol{B}$. thuringiensis on PRM agar. As the incubation time increases from 6 to $8 \mathrm{~h}$, ELS pattern evolves to a fully developed speckle when an average speckle diameter decreases.

number of speckles were generated, and still had concentric circle pattern. As the surface roughness increased, the size of the speckle decreased and higher number of speckles were generated, and formed a random speckle pattern rather than concentric circle pattern as predicted.

Figure 7 shows the comparison of theoretical speckle development on diffraction pattern by surface roughness and that from experiment. The results were plotted for speckle area vs. the number of speckles. For the prediction, the maximum amplitude of the random function for $\Phi_{s}$ were set as $1 / 200,1 / 150$, and $1 / 100$ of $B$. cereus colony center height for fine, medium, and coarse roughness, respectively. As the theoretical model moved from coarse $(\square)$ to fine $(\triangleright)$ surface roughness, the model predicts decreasing average speckle size with increasing numbers. Similar trend was observed in experiment with $B$. cereus ATCC14579 incubated from $6 \mathrm{~h}(\boxminus)$ to $8 \mathrm{~h}(\triangle)$ (Figure 7A). For B. thuringiensis DUP6044, $\Phi_{s}$ was changed to $1 / 180,1 / 130$, and $1 / 100$ of colony center height and an excellent agreement between theory and experiment was evident (Figure 7B). For example, coarse surface roughness $(\boxminus)$ and $9 \mathrm{~h}$ incubation $(\triangle)$ showed almost similar trend in average speckle size and the numbers.

\section{DISCUSSION}

Understanding bacterial growth on a solid surface is important for biophysicist, microbial ecologist, and food microbiologist for better insight of colony development and its ecological and virulence properties. In context to virulence attributes of colony, small colony variants (SCVs) of different bacteria are often considered more virulent facilitating persistence and recurrent infections (Proctor et al., 2006). Harshey (2003) wrote an excellent review on how bacteria form colony in four different ways (swarming, gliding, twitching, and sliding). Our motivation to this study was to correlate the unique growth characteristics of Bacillus species to the optical speckle theory and help understand colony growth dynamics. Bacillus has long been used as a model to study the environment dependent variations in growth patterns (Shimada et al., 1995; Ben-Jacob et al., 1998; Bees et al., 2000; Stecchini et al., 2001; Kaito and Sekimizu, 2007; Pipe and Grimson, 2008). However, recent application of optical scattering-based bacterial colony interrogation requires different perspective for fundamental understanding of scatter pattern generation and colony morphology.

Previous studies have shown that availability of nutrients and agar concentration affect the distribution/propagation of cells within a colony (Bae et al., 2011). However, the published studies on colony scatter pattern did not report theoretical interpretation of speckle pattern formation by a colony. To the best of our knowledge, this is the first report of theoretical interpretation of speckle pattern formation by Bacillus colony, which is largely attributed to the roughness of bacterial colony. The surface roughness is believed to be arisen from the swarming behavior of the Bacillus cells during colony formation. ICMA and PCM measurements 
revealed that $B$. subtilis generates more rough surface morphology than the B. polymyxa (Figures 2, 3).

For theoretical comparison, ICMA measurement was critical since the scalar diffraction theory required accurate description of the amplitude and phase modulation component of Equations (1)-(11). Both qualitative trend and quantitative measurement showed good agreement (Figure 7). Both theoretical model and the experimental evidence indicate that as bacteria grow, the speckle size decreases and the number increases. Furthermore, the quantitative comparison also revealed that as surface roughness changed from fine to coarse, the number of speckle increased 7
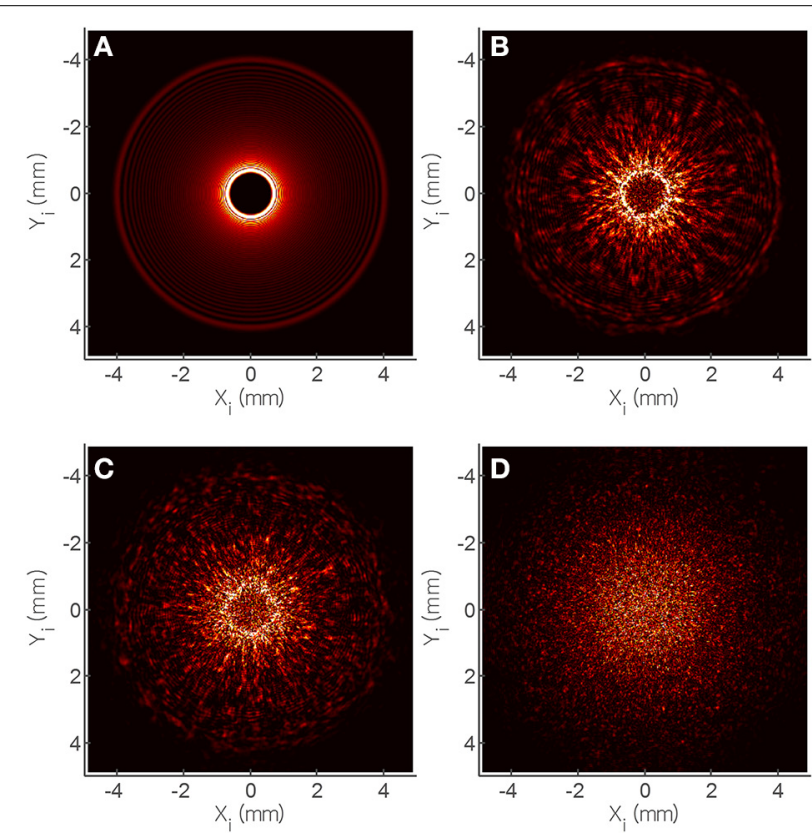

FIGURE 6 | Theoretical analysis of speckle development based on the random phase shift $\left(\varphi_{\mathrm{s}}\right)$ of scalar diffraction theory. Phase shift was modified as (A) $1 / 200$, (B) $1 / 150$, (C) $1 / 100$, and (D) $1 / 80$ and their simulated scatter patterns show similar trend with the experiment.
(B. cereus) to 10 (B. thuringiensis) -fold. Similar quantitative trend was observed for two other bacterial species: $B$. cereus and $B$. thuringiensis. In future, this information could be used to provide fundamental understanding of inverse scattering method for analyzing bacterial colony formation since size and the number of speckle correlate with incubation time and surface roughness.

The light scattering sensor discussed in this study was successfully used for detection of bacterial pathogens including Listeria monocytogenes (Banada et al., 2007, 2009), Vibrio spp. (Huff et al., 2012), Salmonella serovars (Singh et al., 2014), E. coli O157:H7 (Tang et al., 2014), and Bacillus spp. (Singh et al., submitted). However, these studies did not investigate if mathematical modeling could be used to explain resulting scatter patterns of colonies. Here we focused on developing and comparing the computational model for B. subtitis ATCC 6633 and B. polymyxa B719W for speckle formation and its resultant effect on the experimental scatter pattern. Such in silco study would be of great significance for extrapolating the colonial properties of highly virulent Bacillus species such as Bacillus anthracis, a causative agent of anthrax that requires a high containment facility for handling.

Rather than utilizing specific labeling reagents, proposed method adopted the combination of a laser light and a 2D CMOS sensor to transform the both 3-D macroscopic and microscopic structures (morphology) and material characteristics (refractive indices) effect into a single 2-D scatter image which can be further analyzed by chemometric methods. The proposed method does not disrupt the colony structure while capturing the scatter pattern, thus can be further used in biochemical, molecular, immunological and mass-spectroscopic methods for confirmation of the cultures.

As the modeling of forward scattering pattern for the bacteria colony shows, the morphology and optical characteristics play major roles into formation of diffraction patterns. Previous studies have shown that availability of nutrients and agar concentration (Bae et al., 2011), and storage condition of the agar plate (Mialon et al., 2012) possibly affect the distribution/propagation of cells within a colony and the resulting diffraction pattern. Furthermore, previous studies (Bae et al., 2007, 2010; Kim et al., 2013) indicate that the forward scattering patterns produced by

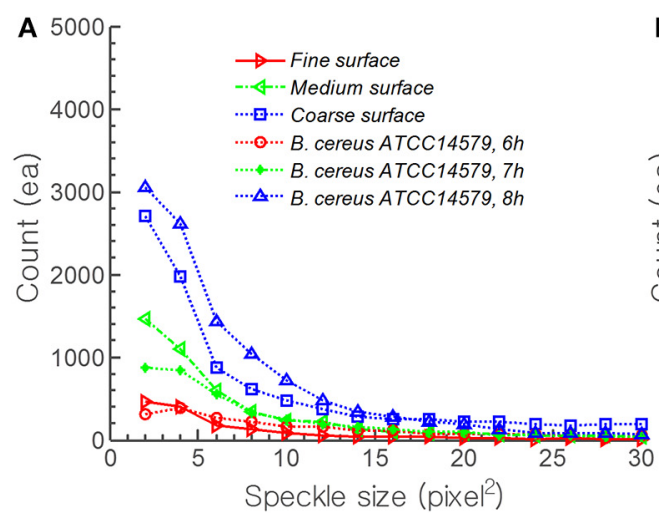

FIGURE 7 | Comparison of theoretical speckle development on diffraction pattern by surface roughness and that of experimental time resolved result for $\boldsymbol{B}$. cereus and $\boldsymbol{B}$. thuringiensis. For prediction, the

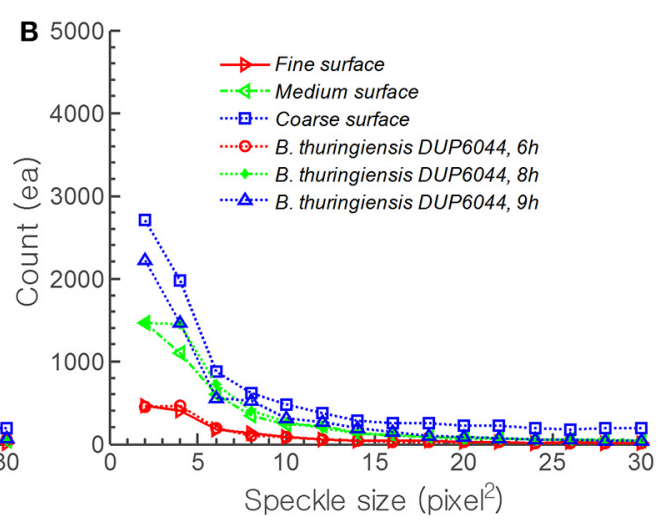

maximum amplitude of random function of surface roughness was adopted as (A) $1 / 200,1 / 150$, and $1 / 100$ (B) $1 / 180,1 / 130$, and $1 / 100$ of colony center height for fine, medium, and coarse surface roughness, respectively. 
BARDOT are circular symmetric or concentric circle with some radial spokes for most of the microorganisms (E. coli, Listeria, Salmonella, and Staphylococcus) studied.

The outcome of this study would be helpful in analyzing the growth characteristic and understanding of the colonial behavior on solid agar surface using visual, physical, mathematical and empirical models. The results will also help in development of better scatter signatures based classification algorithm for microbial detection, especially the pathogens and spoilage microbes relevant to food safety, food quality and food security.

\section{CONCLUSION}

Here we report a theoretical modeling and experimental verification of the swarming growth characteristics of Bacillus species using optical scattering technology. To quantify the growth characteristics, PCM and ICMA along with BARDOT were utilized. Scalar diffraction theory coupled with new random phase component provided good agreement with experimental speckle pattern formation. The results indicate that as bacteria grow, their average speckle size decrease and the number of smaller speckle increases due to the swarming growth characteristics of bacteria within the colony.

\section{ACKNOWLEDGMENT}

This research was supported through a cooperative agreement with the Agricultural Research Service of the US Department of Agriculture project number 1935-42000-035 and the Center for Food Safety Engineering at Purdue University.

\section{REFERENCES}

Bae, E., Aroonnual, A., Bhunia, A. K., and Hirleman, E. D. (2011). On the sensitivity of forward scattering patterns from bacterial colonies to media composition. J. Biophotonics 4, 236-243. doi: 10.1002/jbio.201000051

Bae, E., Bai, N., Aroonnual, A., Robinson, J. P., Bhunia, A. K., and Hirleman, E. D. (2010). Modeling light propagation through bacterial colonies and its correlation with forward scattering patterns. J. Biomed. Opt. 15:045001. doi: $10.1117 / 1.3463003$

Bae, E., Banada, P. P., Huff, K., Bhunia, A. K., Robinson, J. P., and Hirleman, E. D. (2007). Biophysical modeling of forward scattering from bacterial colonies using scalar diffraction theory. Appl. Opt. 46, 3639-3648. doi: 10.1364/AO.46.003639

Banada, P. P., Guo, S. L., Bayraktar, B., Bae, E., Rajwa, B., Robinson, J. P., et al. (2007). Optical forward-scattering for detection of Listeria monocytogenes and other Listeria species. Biosens. Bioelectron. 22, 1664-1671. doi: 10.1016/j.bios.2006.07.028

Banada, P. P., Huff, K., Bae, E., Rajwa, B., Aroonnual, A., Bayraktar, B., et al. (2009). Label-free detection of multiple bacterial pathogens using light-scattering sensor. Biosens. Bioelectron. 24, 1685-1692. doi: 10.1016/j.bios.2008.08.053

Bees, M. A., Andresen, P., Mosekilde, E., and Givskov, M. (2000). The interaction of thin-film flow, bacterial swarming and cell differentiation in colonies of Serratia liquefaciens. J. Math. Biol. 40, 27-63. doi: 10.1007/s002850050004

Ben-Jacob, E., Cohen, I., and Gutnick, D. L. (1998). Cooperative organization of bacterial colonies: from genotype to morphotype. Annu. Rev. Microbiol. 52, 779-806. doi: 10.1146/annurev.micro.52.1.779

Cohen, I., Golding, I., Kozlovsky, Y., Ben-Jacob, E., and Ron, I. G. (1999). Continuous and discrete models of cooperation in complex bacterial colonies. Fractals 7, 235-247. doi: 10.1142/S0218348X99000244

Granek, J. A., and Magwene, P. M. (2010). Environmental and Genetic determinants of colony morphology in Yeast. PLoS Genet. 6:e1000823. doi: 10.1371/journal.pgen.1000823

Harshey, R. M. (2003). Bacterial motility on a surface: many ways to a common goal. Annu. Rev. Microbiol. 57, 249-273. doi: 10.1146/annurev.micro.57.030502. 091014
Henegariu, O., Heerema, N. A., Dlouhy, S. R., Vance, G. H., and Vogt, P. H. (1997). Multiplex PCR: critical parameters and step-by-step protocol. Biotechniques 23, 504-511.

Huff, K., Aroonnual, A., Littlejohn, A. E. F., Rajwa, B., Bae, E., Banada, P. P., et al. (2012). Light-scattering sensor for real-time identification of Vibrio parahaemolyticus, Vibrio vulnificus and Vibrio cholerae colonies on solid agar plate. Microb. Biotechnol. 5, 607-620. doi: 10.1111/j.1751-7915.2012. 00349.x

Kaito, C., and Sekimizu, K. (2007). Colony spreading in Staphylococcus aureus. J. Bacteriol. 189, 2553-2557. doi: 10.1128/JB.01635-06

Kawasaki, K., Mochizuki, A., Matsushita, M., Umeda, T., and Shigesada, N. (1997). Modeling spatio-temporal patterns generated by Bacillus subtilis. J. Theor. Biol. 188, 177-185. doi: 10.1006/jtbi.1997.0462

Keyence-Corporation. (2006). Surface Scanning Laser Confocal Displacement Meter LT-9001 Series. Osaka: Keyence Corporation.

Kim, H., Bai, N., Bhunia, A. K., King, G. B., Hirleman, E. D., and Bae, E. (2013). Development of an integrated optical analyzer for characterization of growth dynamics of bacterial colonies. J. Biophotonics 6, 929-937. doi: 10.1002/jbio.201200224

Kozlovsky, Y., Cohen, I., Golding, I., and Ben-Jacob, E. (1999). Lubricating bacteria model for branching growth of bacterial colonies. Phys. Rev. E 59, 7025-7035. doi: 10.1103/PhysRevE.59.7025

Kuo, W. S., and Chak, K. F. (1996). Identification of novel cry-type genes from Bacillus thuringiensis strains on the basis of restriction fragment length polymorphism of the PCR-amplified DNA. Appl. Environ. Microbiol. 62, 1369-1377.

Lega, J., and Passot, T. (2003). Hydrodynamics of bacterial colonies: a model. Phys. Rev. E 67, (3 Pt 1):031906. doi: 10.1103/PhysRevE.67.031906

Lorenz, T. C. (2012). Polymerase chain reaction: basic protocol plus troubleshooting and optimization strategies. J. Vis. Exp. e3998. doi: 10.3791/3998.

Manzano, M., Giusto, C., Iacumin, L., Cantoni, C., and Comi, G. (2003). A molecular method to detect Bacillus cereus from a coffee concentrate sample used in industrial preparations. J. Appl. Microbiol. 95, 1361-1366. doi: 10.1046/j.1365-2672.2003.02120.x

Marcoux, P., Dupoy, M., Cuer, A., Kodja, J.-L., Lefebvre, A., Licari, F., et al. (2014). Optical forward-scattering for identification of bacteria within microcolonies. Appl. Microbiol. Biotechnol. 98, 2243-2254. doi: 10.1007/s00253-013-5495-4

Merkus, H. G. (2009). Particle Size Measurements: Fundamentals, Practice, Quality. Osaka: Springer Science \& Business Media. ISBN: 9781402090165. doi: 10.1007/978-1-4020-9016-5

Mialon, M., Tang, Y., Singh, A. K., Bae, E., and Bhunia, A. K. (2012). Effects of preparation and storage of agar media on the sensitivity of bacterial forward scattering patterns. Open J. Appl. Biosens. 1, 26-35. doi: 10.4236/ojab.2012. 13004

Nagai, S., Nishizawa, Y., Onodera, M., and Aiba, S. (1971). Effect of dissolved oxygen on growth yield and aldolase activity in chemostat culture of Azotobacter vinelandii. J. Gen. Microbiol. 66, 197-203. doi: 10.1099/00221287-66-2-197

Ngamwongsatit, P., Banada, P. P., Panbangred, W., and Bhunia, A. K. (2008). WST1-based cell cytotoxicity assay as a substitute for MTT-based assay for rapid detection of toxigenic Bacillus species using CHO cell line. J. Microbiol. Methods 73, 211-215. doi: 10.1016/j.mimet.2008.03.002

Pipe, L. Z., and Grimson, M. J. (2008). Spatial-temporal modelling of bacterial colony growth on solid media. Mol. Biosyst. 4, 192-198. doi: 10.1039/ b708241j

Proctor, R. A., von Eiff, C., Kahl, B. C., Becker, K., McNamara, P., Herrmann, M., et al. (2006). Small colony variants: a pathogenic form of bacteria that facilitates persistent and recurrent infections. Nat. Rev. Microbiol. 4, 295-305. doi: 10.1038/nrmicro1384

Shapiro, J. A. (1992). Pattern and control in bacterial colony development. Sci. Prog. 76, 399-424.

Shimada, Y., Nakahara, A., Matsushita, M., and Matsuyama, T. (1995). Spatiotemporal patterns produced by bacteria. J. Phys. Soc. Jpn. 64, 1896-1899. doi: 10.1143/JPSJ.64.1896

Singh, A. K., Bettasso, A. M., Bae, E., Rajwa, B., Dundar, M. M., Forster, M. D., et al. (2014). Laser optical sensor, a label-free on-plate Salmonella enterica colony detection tool. mBio 5:e01019-13. doi: 10.1128/mBio.01019-13

Stecchini, M. L., Del Torre, M., Donda, S., Maltini, E., and Pacor, S. (2001). Influence of agar content on the growth parameters of Bacillus cereus. Int. J. Food Microbiol. 64, 81-88. doi: 10.1016/S0168-1605(00)00436-0 
Suchwalko, A., Buzalewicz, I., Wieliczko, A., and Podbielska, H. (2013). Bacteria species identification by the statistical analysis of bacterial colonies Fresnel patterns. Opt. Express 21, 11322-11337. doi: 10.1364/OE.21.011322

Tang, Y., Kim, H., Singh, A. K., Aronnual, A., Bae, E., Rajwa, R., et al. (2014). Light scattering sensor for direct identification of colonies of Escherichia coli serogroups O26, O45, O103, O111, O121, O145 and O157. PLoS ONE 9:e105272. doi: 10.1371/journal.pone.0105272

Vliet, L. J. V. (2014). DIPimage and DIPlib Ver. 2.6. [Online]. Available online at: http://www.diplib.org/main

Wimpenny, J. W. T. (1992). Microbial systems - patterns in time and space. $A d v$. Microb. Ecol. 12, 469-522. doi: 10.1007/978-1-4684-7609-5_10

Conflict of Interest Statement: Euiwon Bae and Arun K. Bhunia are inventors of "System and method for rapid detection and characterization of bacterial colonies using forward light scattering" (US Patent No. 7465560), designated BARDOT, described in this review. The authors declare that the research was conducted in the absence of any commercial or financial relationships that could be construed as a potential conflict of interest.

Received: 02 August 2014; paper pending published: 01 September 2014; accepted: 26 September 2014; published online: 14 October 2014.

Citation: Kim H, Singh AK, Bhunia AK and Bae E (2014) Laser-induced speckle scatter patterns in Bacillus colonies. Front. Microbiol. 5:537. doi: 10.3389/fmicb. 2014.00537

This article was submitted to Food Microbiology, a section of the journal Frontiers in Microbiology.

Copyright (c) 2014 Kim, Singh, Bhunia and Bae. This is an open-access article distributed under the terms of the Creative Commons Attribution License (CC BY). The use, distribution or reproduction in other forums is permitted, provided the original author(s) or licensor are credited and that the original publication in this journal is cited, in accordance with accepted academic practice. No use, distribution or reproduction is permitted which does not comply with these terms. 


\title{
Microcalorimetric study of the growth of Streptococcus thermophilus in renneted milk
}

\author{
Irina Stulova ${ }^{1,2}{ }^{*}$, Natalja Kabanova $^{2}$, Tiina Kriščiunaite ${ }^{2}$, Kaarel Adamberg ${ }^{1,2}$, Tiiu-Maie Laht ${ }^{1}$ and \\ Raivo Vilu ${ }^{1,2}$
}

${ }^{1}$ Department of Chemistry, Tallinn University of Technology, Tallinn, Estonia

${ }^{2}$ Competence Centre of Food and Fermentation Technologies, Tallinn, Estonia

\section{Edited by:}

Michael Gänzle, Alberta Veterinary

Research Institute, Canada

Reviewed by:

Louis Coroller, Université de Brest,

France

Laurent Guillier, Agence Nationale de Sécurité Sanitaire de

I'Alimentation, de l'Environnement et du Travail, France

*Correspondence:

Irina Stulova, Department of Chemistry, Tallinn University of

Technology, Akadeemia Tee 15,

Tallinn 12618, Estonia

e-mail: irina.stulova@tftak.eu
The growth of Streptococcus thermophilus ST12 (ST12) in liquid milk, reconstituted from low-heat skim milk powder reconstituted skim milk (RSM) and in RSM with rennet addition ( $r-R S M)$ at $40^{\circ} \mathrm{C}$ was monitored by microcalorimetry. It was shown that the growth rate of bacteria decreased in renneted samples in comparison with liquid RSM starting from certain sizes of the colonies ("deviation moments"), which depended on the inoculation rates. The hydrolysis of lactose was delayed for about $1 \mathrm{~h}$ in the r-RSM in comparison with RSM but otherwise the metabolism of carbohydrates in the renneted and non-renneted milks was similar. The total free amino acids (TFAA) content by the end of fermentations was higher in r-RSM than in RSM presumably due to the enzymatic hydrolytic activity of rennet. The quantitatively dominating amino acids were remarkably different in the r-RSM and RSM indicating that the hydrolysis cascade of caseins and/or metabolism of amino acids by the bacteria functioned differently in the two cases. The data obtained showed potential of microcalorimetry to characterize quantitative differences of growth and metabolism of the bacteria in renneted and liquid samples of milk.

Keywords: Streptococcus thermophilus, growth and metabolism of the bacteria, milk, renneted milk, microcalorimetry

\section{INTRODUCTION}

Studies of growth of LAB in the liquid milk (Favrot and Maubois, 1994, 1996; Letort et al., 2002) and solid cheese are complicated because traditional microbiological methods are not well suited for the enumeration of bacteria in opaque and especially in solid media. Peculiarities of colonial growth of bacteria in solid transparent matrices (agar, gelatine) have been described, however, in several papers (Brocklehurst et al., 1995; McKay et al., 1997; Malakar et al., 2002; Wilson et al., 2002; Kabanova et al., 2009, 2012; Velliou et al., 2013). Studies of growth of LAB in solid coagulated milk samples are clearly less numerous (Favrot and Maubois, 1996; Floury et al., 2010, 2013; Jeanson et al., 2011). One of the reasons of the noted scarcity is the lack of suitable methods for the studies.

The most noticeable advantage of the calorimetric method in comparison with the other mostly invasive techniques is the possibility to follow the bacterial growth in opaque and/or solid environments like various food matrices monitoring the heat evolution without destroying or perturbing samples studied (Lobete et al., in press). The calorimetric technique was first used in dairy research in seventies for milk grading (Berridge et al., 1974) and growth studies of pure LAB cultures under limiting substrate conditions (Fujita et al., 1978). The interactions of Streptococcus thermophilus and Lactobacillus bulgaricus were studied in milk using calorimetry in 1979 (Monk, 1979). Riva et al. (1997) investigated shelf life of fresh milk using isothermal calorimetry for continuous monitoring of microbial growth. Gardea et al. (2002) compared heat evolution by bacteria with the results of traditional plate counts assessing microbiological quality of milk undergone different treatments. Wadsö and Galindo (2009) compared the thermal power-time profiles of the fermentation of milk at two incubation temperatures using two different buttermilk cultures. Kriščiunaite et al. (2011) applied microcalorimetry to study the influence of $\mathrm{H}_{2} \mathrm{O}_{2}$ on the growth of thermophilic starter bacteria in UHT milk.

A considerable methodical improvement in use of microcalorimetry was introduced in Kabanova et al. (2009) and Kabanova et al. (2012). Two major advancements introduced were as follows: (a) a method of serial dilution was developed, which means that inoculation rates of the bacteria in the samples were changed from $10^{0}$ to $10^{6} \mathrm{CFU} \mathrm{mL} \mathrm{mL}^{-1}$ with the precisely ten-fold increment increment. This allows to study the growth of the populations and colonies of different numbers of cells, and (b) a number of additional samples prepared in parallel with the microcalorimetric samples are incubated at the same temperature, and they are used for the measurements of sugars, organic and amino acids etc. The data obtained from the study of these parallel samples is used together with the microcalorimetric data. The approach was applied for the characterization of peculiarities of the growth of Streptococcus thermophilus ST12 in differently pretreated milk samples (Stulova et al., 2011), and in milk reconstituted from non-irradiated and irradiated at $10 \mathrm{kGy}$ milk powder (Stulova et al., 2013). 
The approach developed was used also in this paper. The specific aim of the present study was to investigate the growth of Streptococcus thermophilus ST12 in renneted reconstituted skim milk (RSM) (in milk gel) and compare the peculiarities of the growth with those in liquid milk samples.

\section{MATERIALS AND METHODS PREPARATION OF RECONSTITUTED SKIM MILK (RSM)}

Low heat skim milk powder (LHSMP) was obtained from Valio Ltd. (Helsinki, Finland). LHSMP was suspended in distilled water to yield a final concentration of $10 \%\left(\mathrm{w} \mathrm{v}^{-1}\right)$ milk solids, with addition of $\mathrm{CaCl}_{2}$ in final concentration of $10 \mathrm{mM}$. The samples with LHSMP were mixed thoroughly for $1 \mathrm{~h}$ at room temperature, heated at $90^{\circ} \mathrm{C}$ for $30 \mathrm{~min}$ and cooled to $30^{\circ} \mathrm{C}$ immediately before the experiments and used as liquid RSM samples.

\section{BACTERIAL CULTURES AND PREPARATION OF INOCULUM}

The strain of Streptococcus thermophilus ST12 (further St. thermophilus ST12) was kindly provided by Chr. Hansen (Hørsholm, Denmark). Deep-frozen cultures of St. thermophilus ST12 were thawed and pre-grown on M17 agar (LAB M, Lancashire, UK) for $24 \mathrm{~h}$ at $40^{\circ} \mathrm{C}$. One colony from a M17 agar plate was inoculated into $10 \mathrm{~mL}$ of RSM and left at $40^{\circ} \mathrm{C}$ until coagulation $(16 \mathrm{~h})$. One per cent of the culture was used for inoculation of the $10 \mathrm{~mL}$ of RSM samples, left until coagulation $(12 \mathrm{~h})$ and further used for inoculation of the microcalorimetry samples. The number of viable cells in the inocula was determined by plating on M17 agar followed by incubation for $72 \mathrm{~h}$ at $40^{\circ} \mathrm{C}$ to be $(1.15 \pm 0.19) \times$ $10^{9} \mathrm{CFU} \mathrm{mL}^{-1}$.

\section{PREPARATION OF SAMPLES FOR GROWTH EXPERIMENTS}

RSM as growth media were prepared in $50 \mathrm{~mL}$ Erlenmeyer flasks. Milk samples were inoculated with $1 \%\left(\mathrm{v} \mathrm{v}^{-1}\right)$ of inoculum and stirred thoroughly. The concentrations of inocula in milk samples for microcalorimetry were varied from $10^{1}$ to $10^{6} \mathrm{CFU} \mathrm{mL} \mathrm{m}^{-1}$ with the 10-fold increment. Milk gels were prepared by addition of $100 \mu \mathrm{l}$ of a $10 \mathrm{~g} \mathrm{~L}^{-1}$ chymosin (CHY-MAX Powder Extra, Hørsholm, Denmark) aqueous solution to $10 \mathrm{~mL}$ of RSM. The enzyme (chymosin) was added immediately after the inoculation. Milk samples for the microcalorimetric experiments, $2 \mathrm{~mL}$ samples of RSM and r-RSM were transferred into the autoclaved microcalorimetric ampoules, inserted into microcalorimeter and maintained at $40^{\circ} \mathrm{C}$. Monitoring of the growth of microorganisms was started in microcalorimeter about $1 \mathrm{~h}$ after the inoculation, after the temperature of the samples was in perfect equilibrium with the set temperature of the thermostat $\left(40^{\circ} \mathrm{C}\right)$. Taking into account that the coagulation of the liquid milk samples was over in $40 \mathrm{~min}$ after the inoculation, the growth of the bacteria in case of the r-RSM samples was studied in practice in solid gel matrices from the beginning of the microcalorimetric monitoring.

The remaining after the preparing of the microcalorimetric samples inoculated and renneted milks were divided into $1 \mathrm{~mL}$ aliquots and incubated at $40^{\circ} \mathrm{C}$ in parallel with the samples in microcalorimeter and used for the determination of $\mathrm{pH}$ and the concentrations of carbohydrates, lactic acid and amino acids at appropriate time points, determined based on the peculiarities of power-time curves.

\section{MICROCALORIMETRY}

A 24 channel isothermal batch microcalorimeter TAM III Thermal Activity Monitor (TA Instruments, New Castle, DE, USA) was used for the monitoring of the growth of thermophilic starter St. thermophilus ST12. Data acquisition and analysis was carried out using TAM Assistant Program (v 0.9.1012.40, SciTech Software AB, Thermometric AB).

\section{Analysis of calorimetric power-time curves and calculation of growth characteristics}

The power-time curves, the output of microcalorimeter describe the heat evolution during the processes studied. A calorimetric

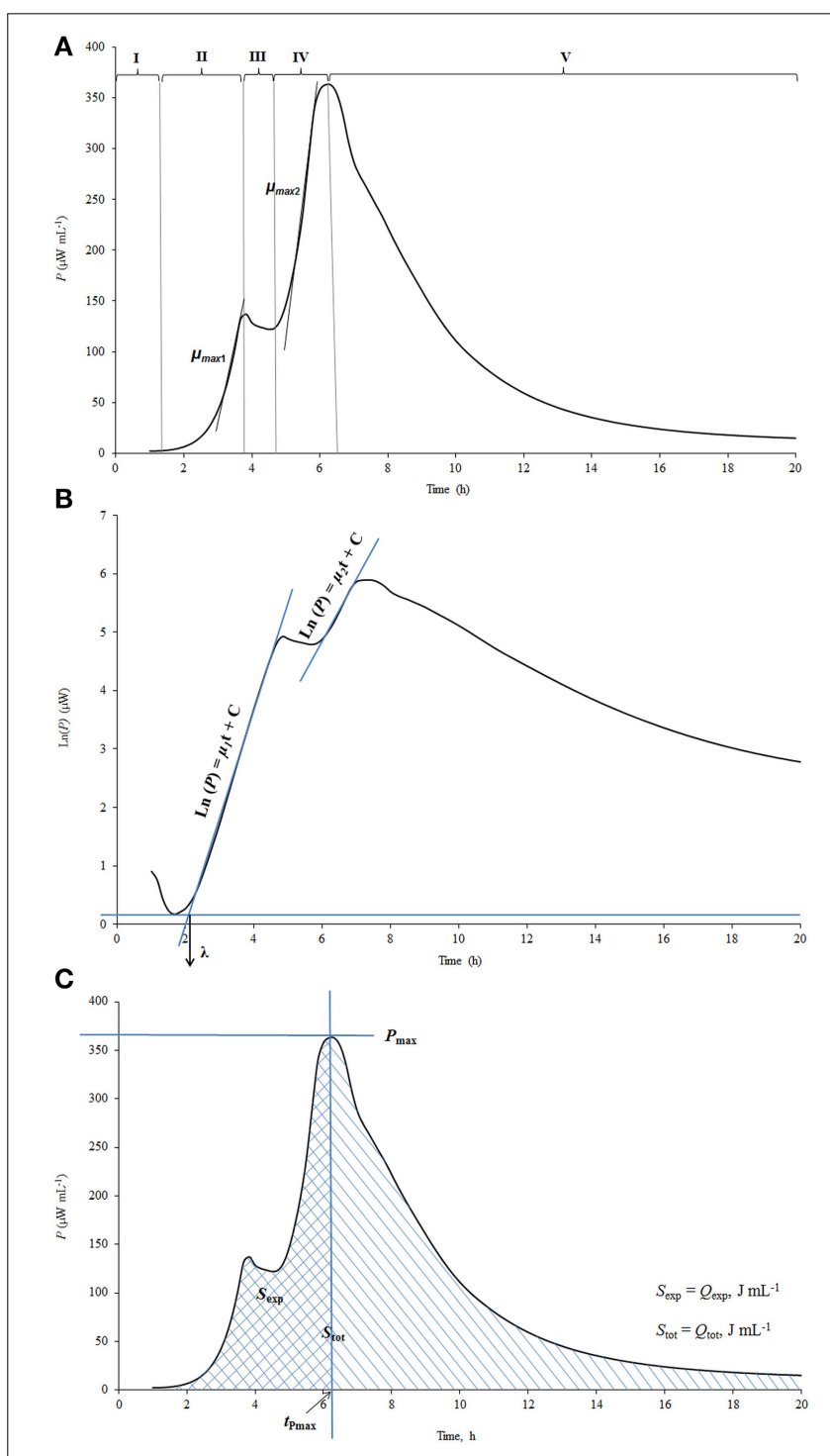

FIGURE 1 | The explanation of the processing of calorimetric power-time curves. (A) The division of calorimetric power-time curves into five phases; (B) Determination of the maximal calorimetric growth rates during the first $\left(\mu_{\max }, \mathrm{Wh}^{-1}\right)$ and the second exponential phase $\left(\mu_{\max 2}\right.$, $\left.\mathrm{Wh}^{-1}\right)$, and the duration of the lag-phase $(\lambda, \mathrm{h})$; (C) Determination of the heat evolution during combined exponential phases $\left(Q_{\text {exp }}\right)$, and during the growth of the cultures $\left(Q_{\text {tot }}\right)$. 
power-time curve of the growth of St. thermophilus ST12 in milk measured by us (Stulova et al., 2013) was divided into five different growth phases as shown in Figure 1A.

The pregrowth phase after the inoculation in the beginning of the graph is characterized as the lag-phase (I) during which bacterial cells adapt to the new environment. The length of the lag-phase was determined as shown on Figure 1B, where the power-time curve is presented in semilog scale. It is important to note that in measuring of the length of the lag-phase on the basis of calorimetric curves the sensitivity of the instrument TAM III $\left(7 \times 10^{-4} \mathrm{~J} / 0.5 \mu \mathrm{W}\right)$ as well as the time during which heat produced by the cells exceeds the level of sensitivity of the instrument should be taken into account (see Kabanova et al., $2009,2012)$. The second phase of the power-time curves following the lag-phase corresponds to the first exponential growth phase (II) during which the bacteria grow at the maximal calorimetric growth rate $\mu_{\max }$ possible in the experimental conditions studied. During the intermediate non-exponential growth phase (III) lactic acid bacteria presumably synthesize proteinases and metabolism of this bacteria switch from growth on free amino acids (FAA) present in milk to growth on amino acids and peptides released by the enzymes synthesized (Letort et al., 2002). The ends of the first as well as the second exponential phases (IV) of bacterial growth were defined by the corresponding peaks of the power-time curves. In case of analysis of certain metabolic characteristics of the bacteria during the growth (consumption patterns of amino acids etc.) the two exponential phases were considered as one-see below. After the second exponential growth phase the deceleration phase $(\mathrm{V})$ which combines the stationary phase of the growth together with the deceleration of the metabolism of lactic acid bacteria growing in milk was taking place.

Maximum specific growth rate $\left(\mu_{\max }\right)$, which could be considered also as maximal calorimetric growth rate during the first and second exponential growth phases was measured as shown in Figure 1B. Taking into account that during the exponential growth phase the relationship between biomass concentration

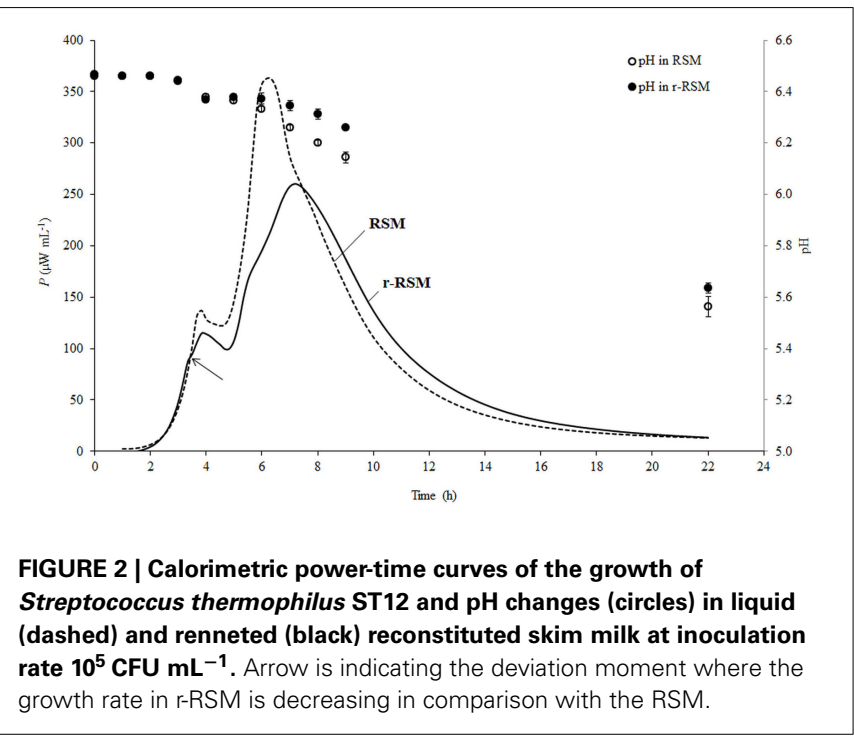

$(X)$ and specific growth rate $(\mu)$ may be described by the first order kinetics:

$$
\frac{d X}{d t}=\mu X
$$

and assuming that the rate of biomass formation $(\mathrm{d} X / \mathrm{d} t)$ was proportional to the rate of heat production $(\mathrm{dQ} / \mathrm{d} t)$, the maximum specific growth rate $\left(\mu_{\max }\right)$ was calculated from the power-time curves as a slope of $\ln \mathrm{d} Q / \mathrm{d} t$ over time $(t)$, as shown in Maskow and Babel (2003):

$$
\ln \frac{d Q}{d t_{t}}=\frac{d Q}{d t_{0}}+\mu_{\max } t
$$

The heat produced during the exponential growth phase $Q_{\exp }$ (area between the power-time curve and the vertical line that goes through the major peak of the power-time curve $\left(P_{\max }\right.$ value) and the baseline-see Figure $\mathbf{~ C}$ and the total heat produced during the whole process of growth $Q_{\text {tot }}$ (area between the power-time curve and baseline) were determined using the TAM Assistant program (v 0.9.1012.40, SciTech Software AB, Thermometric $\mathrm{AB}$, Järfälla, Sweden) and Microsoft Excel. The recording of the heat evolution was taking place with the frequency $1 \mathrm{~s}^{-1}$.

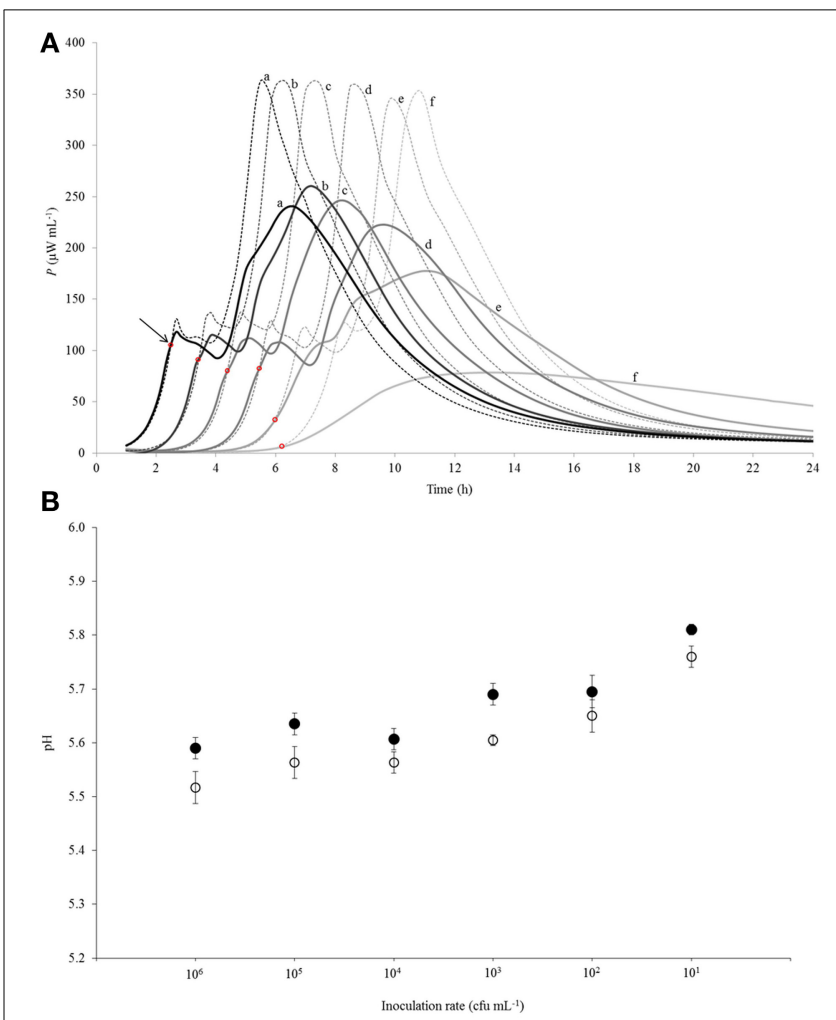

FIGURE 3 | (A) Calorimetric power-time curves describing growth of Streptococcus thermophilus ST12 in liquid (dashed line) and renneted (bold line) reconstituted skim milk at different inoculation rates (CFU $\mathrm{mL}^{-1}$ ): (a) $10^{6}$; (b) $10^{5}$; (c) $10^{4}$; (d) $10^{3}$; (e) $10^{2}$; (f) $10^{1}$. The arrow and the red circles mark the "deviation moments." (B) $\mathrm{pH}$ at the end of fermentation (at $22 \mathrm{~h}$ ) at all inoculation rates $\left(10^{6}-10^{1} \mathrm{CFU} \mathrm{mL}{ }^{-1}\right.$ ) in RSM (white circles) and in $\mathrm{r}$-RSM (black circles). Initial $\mathrm{pH}=6.47$ was the same in the samples. 
The time-points were resampled at equidistant intervals of $15 \mathrm{~min}$.

The value $P_{\max }$ is characterizing the maximum aggregate rate of heat production (see Figure 1C). After reaching the maximum, the decrease of heat production rate starts. The time point where $P_{\max }$ is measured is a convenient time for the end of the exponential growth phase $(\mathrm{s})$.

The change of the number of viable cells over time was calculated using the equation (Stulova et al., 2013):

$$
N=\frac{\Delta Q}{Y Q}
$$

where $N\left(\mathrm{CFU} \mathrm{mL} \mathrm{mL}^{-1} \mathrm{~h}^{-1}\right)$ is the number of bacteria grown during the selected time interval, $\Delta Q\left(\mathrm{~J} \mathrm{~mL}^{-1} \mathrm{~h}^{-1}\right)$ is the heat produced during the same time interval, and $Y_{Q}\left(\mathrm{~J} \mathrm{CFU}^{-1}\right)$ is the experimentally determined heat yield coefficient $\left(Y_{Q}, \mathrm{~J}\right.$ $\left.\mathrm{CFU}^{-1}\right)$. Heat yield coefficient $\left(Y_{Q}, \mathrm{~J} \mathrm{CFU}^{-1}\right)$ was calculated on the basis of the results obtained from the experiments of ST12 growth in liquid RSM. The plate count was carried out at the end of exponential growth phase $\left(6.31^{*} 10^{8} \mathrm{CFU} \mathrm{mL} \mathrm{m}^{-1}\right)$ and heat amount produced was calculated from the power-time curves $(2.81 \mathrm{~J})$. The value of $Y_{Q}\left(\mathrm{~J} \mathrm{CFU}^{-1}\right)$ was determined to be $Y_{Q}=2.81 \mathrm{~J} / 6.31^{*} 10^{8} \mathrm{CFU} \mathrm{mL}^{-1}=4.45^{*} 10^{-9} \mathrm{~J} \mathrm{CFU}^{-1}$. Our earler experiments (unpublished data) showed that the $Y_{Q}$ value depended remarkably on the temperature of the growth but not on the media composition or inoculation rate. The $Y_{Q}$ value indicated was used for the description of growth in the liquid cultures (RSM) as well as in the case of solid-state growth (in r-RSM).

The numbers of bacteria at the end of exponential growth

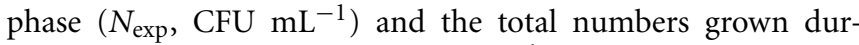
ing the whole process $\left(N_{\text {tot }}, \mathrm{CFU} \mathrm{mL} \mathrm{m}^{-1}\right)$, and also at "deviation moments" ( $\left.N^{*}, \mathrm{CFU} \mathrm{mL}^{-1}\right)$-see below, were calculated from heat evolution data $Q_{\exp }\left(\mathrm{J} \mathrm{mL}^{-1}\right)$ and $Q_{\text {tot }}\left(\mathrm{J} \mathrm{mL}^{-1}\right)$, and $Q^{*}$ $\left(\mathrm{J} \mathrm{mL}^{-1}\right)$ using the indicated above $Y_{Q}$ value (Kabanova et al., 2012).

The radii of average spherical model colonies $\left(R_{\text {col }}, \mu \mathrm{m}\right)$ were calculated from average volumes of colonies using Kepler's conjecture of packing of determined by heat measurements numbers of bacteria in colonies (Hsiang, 1993; Hales, 1994)—see a detailed discussion of the use of the conjecture in Kabanova et al. (2012).

Table 1 | Growth characteristics of Streptococcus thermophilus ST12 in RSM and r-RSM at $40^{\circ} \mathrm{C}^{\mathrm{a}}$.

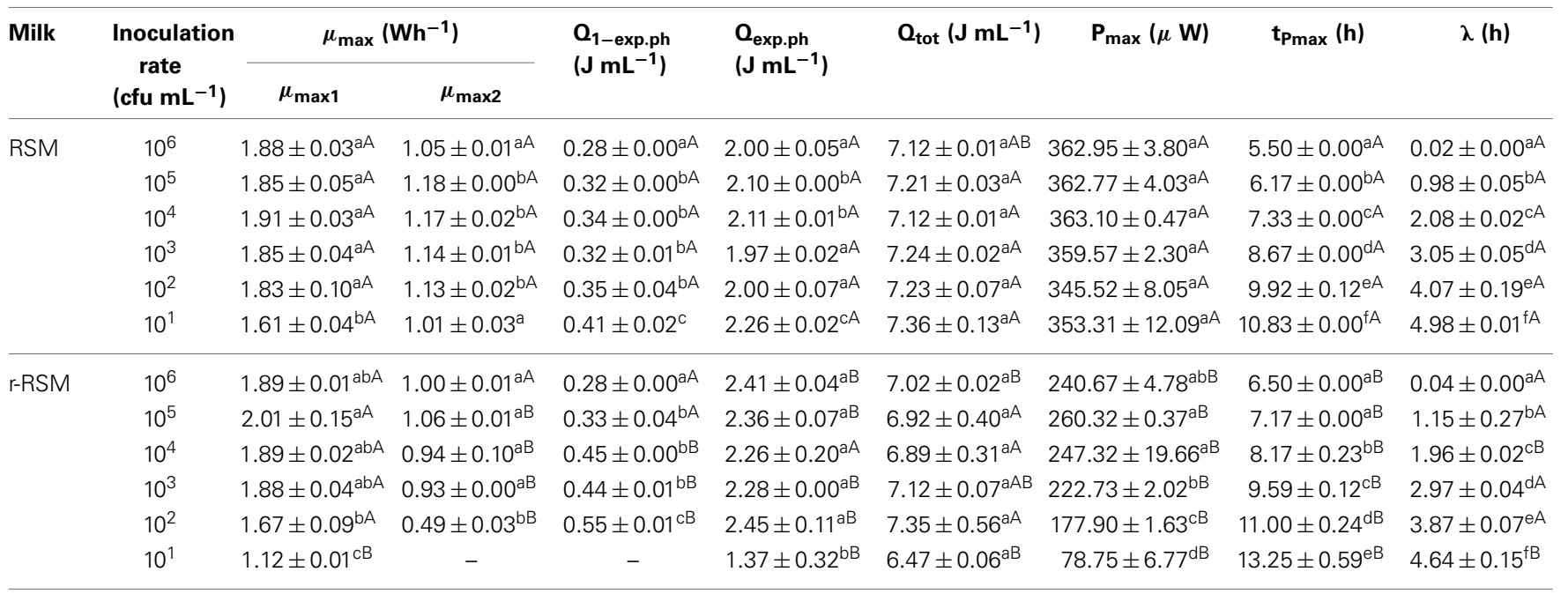

${ }^{a}$ Data are means $\pm S D$ of maximal calorimetric growth rate $\left(\mu_{\max }\right)$ in the first and second exponential growth phase, the heat evolved during the first and second exponential phase $\left(Q_{1 \text { stexp.ph }}\right.$ and $\left.Q_{\text {exp.ph }}\right)$, the total heat produced during the whole fermentation $\left(Q_{\text {tot }}\right)$, maximum heat flow $\left(P_{\text {max }}\right)$, the time of the maximum heat production rate ( $\left.t_{P \max }\right)$ and lag phase duration $(\lambda)$ obtained from microcalorimetric power-time curves.

Table 2 | The $t$-test analysis denoting the differences between liquid and renneted RSM samples.

\begin{tabular}{|c|c|c|c|c|c|c|c|c|c|c|}
\hline Milk & $\begin{array}{l}\text { Inoculation rate } \\
\text { (cfu } \mathrm{mL}^{-1} \text { ) }\end{array}$ & \multicolumn{2}{|c|}{$\mu_{\max }\left(\mathrm{Wh}^{-1}\right)$} & $\begin{array}{l}\mathbf{Q}_{1-\text { exp.ph }} \\
\left(\mathrm{J} \mathrm{mL}^{-1}\right)\end{array}$ & $\begin{array}{l}\mathbf{Q}_{\text {exp.ph }} \\
\left(\mathrm{J} \mathrm{mL}^{-1}\right)\end{array}$ & $\mathrm{Q}_{\text {tot }}\left(\mathrm{J} \mathrm{mL}^{-1}\right)$ & $\mathbf{N}_{\exp \left(\text { cfu } \mathrm{mL}^{-1} \text { ) }\right.}$ & $P_{\max }(\mu \mathrm{W})$ & $t_{P \max (h)}$ & $\lambda(h)$ \\
\hline RSM vs. r-RSM & $10^{6}$ & NS & * & NS & $*$ & * & * & ** & $* * *$ & NS \\
\hline & $10^{5}$ & NS & $* *$ & NS & * & NS & * & $* * *$ & $* * *$ & NS \\
\hline & $10^{4}$ & NS & $* *$ & $* *$ & NS & NS & * & $*$ & $*$ & $*$ \\
\hline & $10^{3}$ & NS & $* *$ & ** & $* *$ & NS & ** & $* * *$ & $* *$ & NS \\
\hline
\end{tabular}

NS-not significant, ${ }^{*} P<0.05,{ }^{* *} P<0.01,{ }^{* *} P<0.001$. 


\section{ANALYTICAL METHODS}

Incubated in parallel to the microcalorimetric samples milk samples were mixed at chosen times 1:1 with isopropanol for sedimentation of proteins. The precipitate was removed by centrifugation at $14,000 \times \mathrm{g}$ for $10 \mathrm{~min}$. The supernatant was filtered through a $13 \mathrm{~mm}$ diameter and $0.2-\mu \mathrm{m}$ pore-size regenerated cellulose (RC) membrane filter (Whatman, Maidstone, UK) and diluted with water before analysis. High-performance liquid chromatography (HPLC) system (Alliance 2695 system, Waters Corp., Milford, MA) with a Refractive Index Detector 2414 and column BioRad HPX-87H $300 \times 7.8 \mathrm{~mm}$ (Hercules, CA) was used for measuring lactose, glucose, galactose and lactate concentrations.

Analysis of FAA was performed on an ultra-performance liquid chromatography (UPLC) system (Acquity UPLC; Waters Corp.) including a binary solvent manager, a sample manager and photodiode array (PDA) detector, connected to Waters Empower $^{\mathrm{TM}} 2.0$ software. Separations were performed on a $2.1 \times$ $100 \mathrm{~mm}$ Waters Acquity UPLC AccQ•Tag Ultra Column operated at $55^{\circ} \mathrm{C}$, the running time being $12 \mathrm{~min}$. Sample derivatization procedure was as follows: $20 \mu \mathrm{L}$ AccQ•Fluor reagent was added to the mixed solution of $70 \mu \mathrm{L}$ AccQ $\bullet$ Fluor borate buffer and $10 \mu \mathrm{L}$ sample or standard. The mixed solution was vortexed immediately for $10 \mathrm{~s}$, transferred to an autosampler vial and allowed to stand at room temperature for $1 \mathrm{~min}$. Then the vials were placed in a heating block at $55^{\circ} \mathrm{C}$ for $10 \mathrm{~min}$, after which they were analyzed by the UPLC system. Empower software (Waters Corp.) was used for the data processing.

The $\mathrm{pH}$ of milk samples was measured with $\mathrm{pH}$ meter S20 Seven Easy equipped with InLab 413 electrode (Mettler-Toledo $\mathrm{GmbH}$, Greifensee, Switzerland).

\section{CALCULATION OF GROWTH PARAMETERS CHARACTERIZING METABOLISM OF THE GROWING CELLS}

The growth characteristics of the bacteria (yield coefficient values, growth rates etc.) during the growth in milk were calculated on the basis of the heat evolution converted to biomass concentrations or cell numbers and concentrations of organic acids etc. in the culture media as follows:

$$
\mu=\frac{\ln \left(N_{2} / N_{1}\right)}{t_{2}-t_{1}}
$$

$$
\begin{aligned}
& I_{\text {Lactose }}=\frac{d\left(C_{\text {Lactose }}\right)}{d(X)} \\
& O_{\text {Lactose }}=\frac{d\left(C_{\text {Lactose }}\right)}{d(X)} \\
& Y_{\text {Lactate Lactose }}=\frac{d\left(C_{\text {Lactate }}\right)}{d\left(C_{\text {Lactose }}\right)} \\
& Y_{X S}=\frac{d(X)}{\left(d\left(2 \cdot C_{\text {Lactose }}\right)-d\left(C_{G a l}\right)-d(G l c)\right) \times 180 / 1000} \\
& I_{A A}=\frac{d\left(C_{A A}\right)}{d(X)}
\end{aligned}
$$

where $\mu$ is the specific growth rate $\left(\mathrm{h}^{-1}\right), \mathrm{t}$ is time $(\mathrm{h}), N$ is the number of cells calculated assuming that heat produced per cell formation is constant $\left(Y_{Q}=4.45 \times 10^{-9} \mathrm{~J}\right.$ per cell $), X$ is the dry biomass ( $\mathrm{gdw} \mathrm{L}^{-1}$ ), assuming that the mass of one cell is $0.2 \times$ $10^{-12} \mathrm{~g}$ (unpublished data), $\mathrm{I}_{\text {Lactose }}$ is the lactose consumption per biomass produced ( $\mathrm{mmol} \mathrm{gdw}^{-1}$ ), $\mathrm{O}_{\text {Lactate }}$ is the lactate production per biomass produced ( $\left.\mathrm{mmol} \mathrm{gdw}^{-1}\right)$; $\mathrm{C}$ designates the concentration of corresponding compound, for example $C_{\text {Lactate }}$ $(\mathrm{mM}), Y_{\text {Lactate/Lactose }}$ shows the lactate yield per lactose consumed, $Y_{\mathrm{XS}}$ is the biomass yield per hexose consumed and $I_{\mathrm{AA}}$

Table 4 | Mean radii of the colonies of Streptococcus thermophilus ST12 in r-RSM at the "deviation moment," at the end of exponential growth phase and at the end of cultivations at different inoculation rates $10^{6}-10^{1} \mathrm{cfu} \mathrm{mL}^{-1}$.

\begin{tabular}{|c|c|c|c|c|c|c|}
\hline \multirow[t]{2}{*}{$\begin{array}{l}\text { Inoculation } \\
\text { rate, cfu } \mathrm{mL}^{-1}\end{array}$} & \multicolumn{2}{|c|}{$\begin{array}{l}\mathrm{N}^{*} \text { at power-time curves "deviation } \\
\text { moment" }\end{array}$} & \multicolumn{2}{|c|}{$\begin{array}{c}N_{\text {exp }} \text { at the end of exponential } \\
\text { phase }\end{array}$} & \multicolumn{2}{|c|}{$\begin{array}{l}\mathrm{N}_{\text {tot }} \text { at the end of fermentation } \\
\text { (at } 22 \mathrm{~h} \text { ) }\end{array}$} \\
\hline & cfu $\mathrm{mL}^{-1}, \times 10^{7}$ & cfu col-1 & cfu $\mathrm{mL}^{-1}, \times 10^{8}$ & cfu col-1 & cfu $\mathrm{mL}^{-1}, \times 10^{9}$ & cfu col-1 \\
\hline $10^{5}$ & $4.96 \pm 0.24$ & $(4.96 \pm 0.24) \times 10^{2}$ & $5.30 \pm 0.16$ & $(5.30 \pm 0.16) \times 10^{3}$ & $1.56 \pm 0.09$ & $(1.56 \pm 0.09) \times 10^{4}$ \\
\hline $10^{4}$ & $4.81 \pm 0.31$ & $(4.81 \pm 0.31) \times 10^{3}$ & $5.08 \pm 0.44$ & $(5.08 \pm 0.44) \times 10^{4}$ & $1.55 \pm 0.07$ & $(1.55 \pm 0.07) \times 10^{5}$ \\
\hline $10^{3}$ & $4.48 \pm 0.90$ & $(4.48 \pm 0.90) \times 10^{4}$ & $5.13 \pm 0.01$ & $(5.13 \pm 0.01) \times 10^{5}$ & $1.60 \pm 0.01$ & $(1.60 \pm 0.01) \times 10^{6}$ \\
\hline $10^{1}$ & $0.94 \pm 0.08$ & $(0.94 \pm 0.08) \times 10^{5}$ & $3.12 \pm 0.73$ & $(3.12 \pm 0.73) \times 10^{7}$ & $1.45 \pm 0.01$ & $(1.45 \pm 0.01) \times 10^{8}$ \\
\hline
\end{tabular}

\begin{tabular}{lccr}
\hline $\begin{array}{c}\text { Inoculation } \\
\text { rate, cfu } \mathbf{~ m L}^{-1}\end{array}$ & $\begin{array}{c}\text { Rcol at } \\
\text { power-time } \\
\text { curves "deviation } \\
\text { moment," } \boldsymbol{\mu ~} \mathbf{m}\end{array}$ & $\begin{array}{c}\text { Rcol at the } \\
\text { end of } \\
\text { exponential } \\
\text { phase, } \boldsymbol{\mu m}\end{array}$ & $\begin{array}{c}\text { Rcol at the } \\
\text { end of } \\
\text { fermentation } \\
\text { (at 22 h), } \boldsymbol{\mu ~ m}\end{array}$ \\
\hline $10^{6}$ & $2.55 \pm 0.01$ & $5.22 \pm 0.02$ & $7.33 \pm 0.01$ \\
$10^{5}$ & $4.94 \pm 0.33$ & $11.16 \pm 0.11$ & $15.95 \pm 0.31$ \\
$10^{4}$ & $10.14 \pm 0.10$ & $23.69 \pm 0.68$ & $33.85 \pm 0.51$ \\
$10^{3}$ & $22.29 \pm 0.36$ & $51.21 \pm 0.02$ & $73.72 \pm 0.22$ \\
$10^{2}$ & $38.33 \pm 0.92$ & $113.05 \pm 1.69$ & $160.40 \pm 4.13$ \\
$10^{1}$ & $64.68 \pm 3.07$ & $200.73 \pm 15.77$ & $331.12 \pm 1.08$ \\
\hline
\end{tabular}

Radii of average colonies were calculated from average volumes of the colony determined using Kepler's conjecture of bacterial packing of colonies.

Table 3 | The numbers of bacteria in the sample (cfu mL-1) and in average colonies (cfu col-1) at the "deviation moment," at the end of

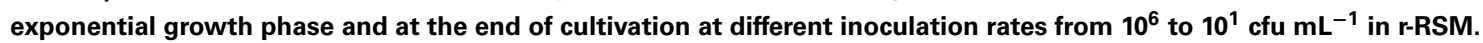

${ }^{*}$ Number of bacteria at the moment of deviation of the microcalorimetric power-time curves obtained in liquid and in renneted reconstituted skim milk samples. 
is the consumption of corresponding amino acid per biomass produced $\left(\mathrm{mmol} \mathrm{gdw}^{-1}\right)$.

\section{STATISTICAL ANALYSIS OF THE DATA}

All the microcalorimetric experiments were repeated twice and measurements were carried out with two or three parallel samples. The power-time curves presented on the Figures and used in the calculations are combined curves of two parallel experiments.

The other analyses (HPLC, UPLC, pH) were carried out in triplicate. All values of the parallel experimental points were averaged and reported along with the value of standard deviation (SD). The experimental data were submitted to single-factor analysis of variance (ANOVA), and the differences of the means were evaluated by Fisher's least significant difference (LSD) test. The difference of the mean values was accepted at the significance level $p<0.05$. The Student's $t$-test was performed to evaluate statistically significant differences between the mean calorimetric values obtained in RSM and r-RSM.

\section{RESULTS}

\section{PECULIARITIES OF THE GROWTH OF ST. THERMOPHILUS ST12 IN} r-RSM

Representative calorimetric power-time curves of the growth of ST 12 in RSM and r-RSM, at inoculation rate $10^{5} \mathrm{CFU} \mathrm{mL}^{-1}$, are presented in Figure 2.

As was previously described (Stulova et al., 2013), a typical microcalorimetric power-time curve of inoculated RSM contained two peaks corresponding to two distinctively separated growth phases (first and second exponential growth phases). As seen in Figure 2, the general pattern with two major exponential growth phases was observed also in r-RSM. The growth curves of St. thermophilus ST12 in r-RSM and RSM were practically coinciding in the beginning, but starting from a certain number of the bacteria in the sample $\left(N^{*}=0.63 \times 10^{7} \mathrm{CFU} \mathrm{mL} \mathrm{m}^{-1}\right.$ at $N_{0}=10^{5}$ $\left.\mathrm{CFU} \mathrm{mL} \mathrm{m}^{-1}\right)$, at the "deviation moment" marked by the arrow in Figure 2 the maximal calorimetric growth rate $\left(\mu_{\max 1}\right)$ in r-RSM

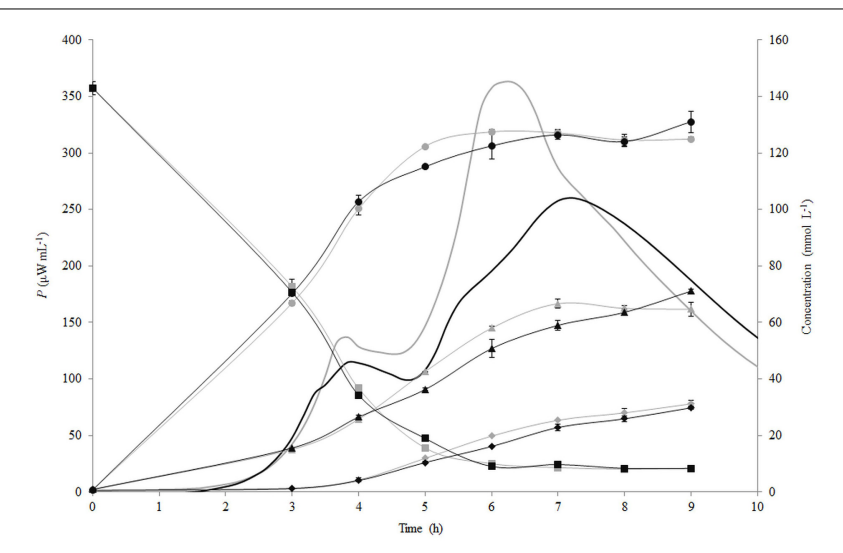

FIGURE 4 | Calorimetric power-time curves (bold line), lactose ( $\square$ ), glucose $(\bullet)$, galactose $(\Delta)$ and lactic acid $(\diamond)$ profiles during the growth of Streptococcus thermophilus ST12 in liquid (gray) and renneted (black) reconstituted milk. was decreased, branching from the curve of RSM, and the further growth in the coagulated matrix was clearly different from that in RSM. This led to the appearance of the second slower phase in the first exponential phase in comparison with the growth curve in milk (RSM). The splitting of the second exponential growth phase also into two was observed as well-see Figure 2.

As seen in Figure 2, acidification developed more slowly after $3.5 \mathrm{~h}$ of incubation in renneted milk compared to the liquid milk samples which was in a good correlation with the slower growth rate of the bacteria in r-RSM. Higher $\mathrm{pH}$ values in r-RSM samples accompanying the main part of the growth of the bacteria were observed in case of all inoculation rates studied (Figure 3B), in difference from a previous report (Favrot and Maubois, 1996) showing slower acidification in rennet curd only at lower level of inoculation $\left(10^{2} \mathrm{CFU} \mathrm{mL}^{-1}\right)$.

\section{Effect of inoculation rate on power-time curves of St. thermophilus ST12 in r-RSM}

Calorimetric power-time curves of the growth of ST 12 in RSM

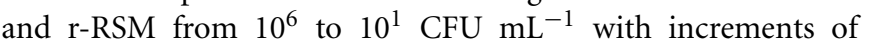
10 -fold, are presented in Figure 3 and processed results were summarized in Table 1. The $t$-test results denoting the differences between liquid and renneted RSM are presented in Table 2.

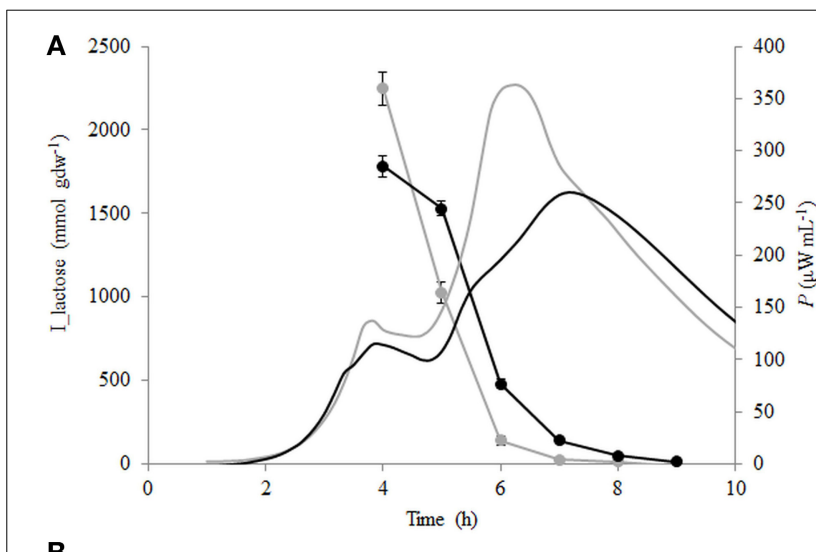

B

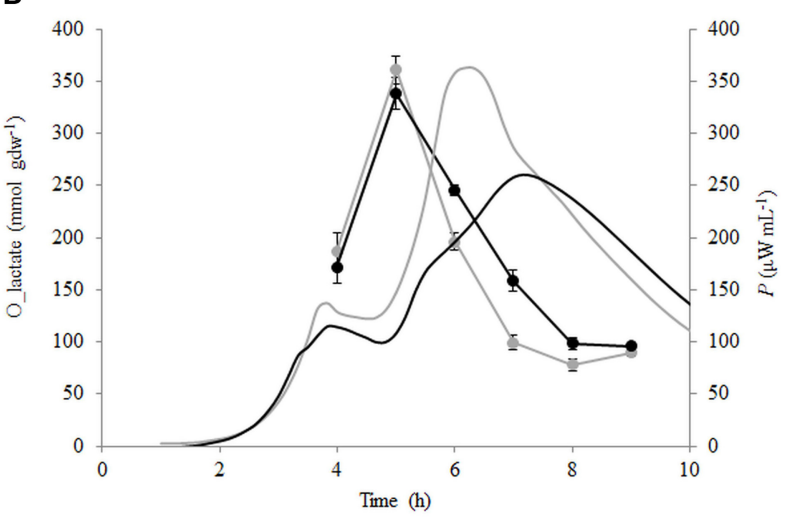

FIGURE 5 | Calorimetric power-time curves (bold line), lactose consumption (A) and lactate production (B) per biomass formed

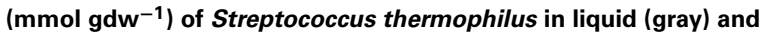
renneted (black) reconstituted milk. 
According to Table 2, the first exponential growth phases of St. thermophilus ST12 in r-RSM were shorter and the calculated $\mu_{\max }$ values were higher than in the second phase like in the case of RSM, however, the noted differences were much more strongly expressed (see also Stulova et al., 2013). As seen from the data presented in Tables 1, 2 the maximal calorimetric growth rates of the first exponential phase $\left(\mu_{\max 1}\right)$ were similar $(P>0.05)$ in RSM and r-RSM at different inoculation rates ranging from $10^{6}$ to $10^{2}$ $\mathrm{CFU} \mathrm{mL} \mathrm{mL}^{-1}$, except $10^{1} \mathrm{CFU} \mathrm{mL}^{-1}$. The values of maximal calorimetric growth rates of the second exponential phase $\left(\mu_{\max 2}\right)$ in r-RSM were significantly lower $(P<0.01)$ than in RSM. This is indicating that the growth of the bacteria in bigger colonies in case of r-RSM is most probably inhibited by the accumulating lactate.

The integrating of the power-time curves allows measure the heat produced by the growing bacteria, where $Q_{\exp }$ indicates the heat produced during the exponential growth phase and $Q_{\text {tot }}$ shows the total heat produced by the total biomass formed during fermentation. According to Table 1 the amounts of heat produced during the exponential phase ( $Q_{\text {exp }}$, average $)$ in r-RSM at inoculation rates ranging $10^{6}-10^{2} \mathrm{CFU} \mathrm{\textrm {mL } ^ { - 1 }}$ were practically the same with average $2.35 \mathrm{~J} \mathrm{~mL}^{-1}$ which corresponds to $5.30 \times 10^{8} \mathrm{CFU}$ $\mathrm{mL}^{-1}$ (calculated using heat yield coefficient $Y_{\mathrm{Q}}$ ). It was about $13 \%$ higher than the same value in $\operatorname{RSM}\left(2.04 \mathrm{~J} \mathrm{~mL}^{-1}\right)$. This fact showed that during the exponential growth phase more bacteria were produced in r-RSM. Noting that the amount of lactate produced during the exponential growth was practically the same in the samples this fact allows to assume that $\mathrm{pH}$ was most probably the factor determining the end of the exponential growth. The amount of heat produced during the total growth $\left(Q_{\text {tot }}\right)$ was the same in r-RSM and RSM at inoculation rates ranging $10^{5}-10^{2}$ $\mathrm{CFU} \mathrm{mL} \mathrm{m}^{-1}-7.14 \pm 0.09 \mathrm{~J} \mathrm{~mL}^{-1}$ that corresponds to the number of bacteria $1.62 \times 10^{9} \mathrm{CFU} \mathrm{mL}^{-1}$. The $Q_{\text {tot }}$ and $N_{\text {tot }}$ values were the lowest in $\mathrm{r}$-RSM at inoculation rate $10^{1} \mathrm{CFU} \mathrm{mL}^{-1}$ $-6.47 \pm 0.06 \mathrm{~J} \mathrm{~mL}^{-1}$ and $1.45 \times 10^{9} \mathrm{CFU} \mathrm{mL} \mathrm{mL}^{-1}$ respectively. This fact showed that growth of the large colonies was clearly inhibited in coagulated milk (but the same amount of bacteria grew without inhibition in RSM).

Clear differences in the $P_{\max }$ values between different inoculation rates in r-RSM are shown in Figure 3. The $P_{\max }$ values were clearly different also if r-RSM and RSM values were compared with each other. As shown in Stulova et al. (2013) the values of $P_{\max }$ were much higher (in average $350 \mu \mathrm{W} \mathrm{mL} \mathrm{m}^{-1}$ ) and very similar at different inoculation rates (see Figure 3 ). The time to reach the maximum heat effect $\left(t_{\mathrm{Pmax}}\right)$ increased with decreasing amounts of inocula (Table 1). As seen from Table 2, there were significant differences noted in $t_{\text {Pmax }}$ between r-RSM and RSM
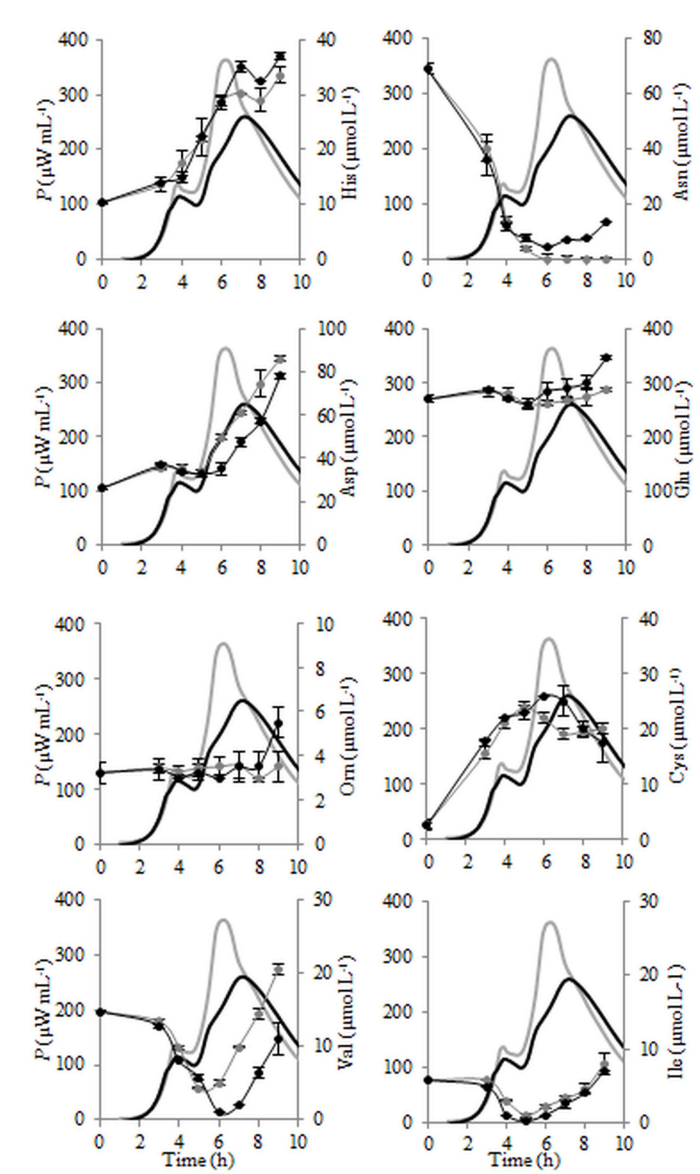
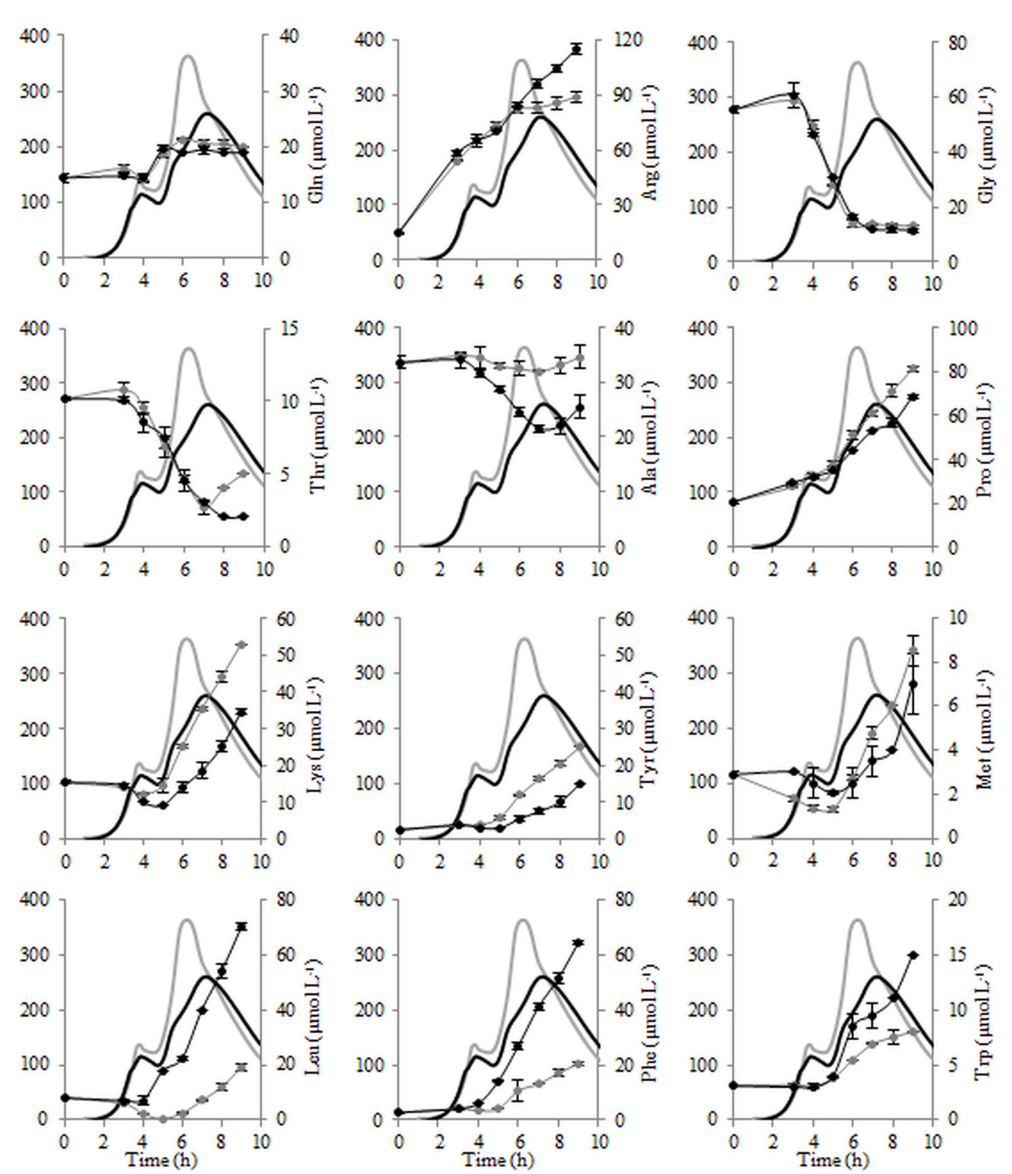

FIGURE 6 | The changes of the concentrations of the individual free amino acids (FAA) during the growth of Streptococcus thermophilus ST12 at inoculation rate $10^{5} \mathrm{CFU} \mathrm{mL} \mathrm{m}^{-1}$ in RSM (gray) and r-RSM (black). 
samples. Depending on the inoculation rate, $1-2.5 \mathrm{~h}$ more time was required to reach the maximum heat production rate $\left(t_{\mathrm{Pmax}}\right)$ in r-RSM compared with RSM (Table 1). These results showed that together with the decrease of the growth rate the length of the exponential phase was increased, which was the expected result.

The most likely explanation for the inhibition of St. thermophilus ST12 growth in r-RSM is that lactate accumulating in the colonies during growth in r-RSM was changing the $\mathrm{pH}$ locally and this led to the inhibiton of growth. The peculiarities of colonial growth of bacteria will be discussed in more detail below (in section Discussion).

Lengths of the lag-phases $(\lambda)$ in r-RSM, as in the case of RSM were changing with regular interval from one inoculation rate to another and became longer on decreasing of the initial inoculum. According to the statistical analysis (Table 2), no big differences were observed in lag-phase $(\lambda)$ durations at the same inoculation rates in both media studied.

\section{Characterization of growth of colonies}

Numbers of bacteria in average colonies in case of different inoculation rates were calculated using the data of the heat evolved during the different phases of the growth and $Y_{\mathrm{Q}}$ value. The radii of the average colonies were calculated using Kepler's conjecture of bacterial packing in colonies (Hsiang, 1993; Hales, 1994). The numbers of bacteria in average colonies (CFU/col) and mean sizes of the average colonies $\left(R_{\mathrm{col}}, \mu \mathrm{m}\right)$ at the "deviation moments," at the end of exponential growth phase and at the end of fermentation at different inoculation rates (from $10^{6}$ to $10^{1} \mathrm{CFU} \mathrm{mL} \mathrm{m}^{-1}$ ) in r-RSM are presented in Tables 3, 4, respectively.

It can be seen from the Table 3 that as expected from the measurements of the heat the total numbers of bacteria in samples at different inoculation rates were practically equal at the end of the cultivations, which automatically means that the grown colonies contained different number of cells. The same was true for the data from the end of the exponential growth.

In agreement with the same type of logic the radii of average colonies at the end of fermentation were varying from $7 \mu \mathrm{m}$ at the highest inoculation rate $10^{6} \mathrm{CFU} \mathrm{mL} \mathrm{m}^{-1}$ to $331 \mu \mathrm{m}$ at

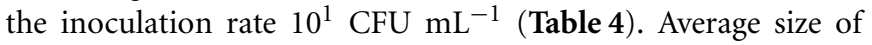
colonies at inoculation rate $10^{5} \mathrm{CFU} \mathrm{mL}^{-1}$ after $22 \mathrm{~h}$ of fermentation was similar to that measured in a model cheese by Floury et al. (2013)—average diameters of colonies being approximately $30 \mu \mathrm{m}$ and $30-50 \mu \mathrm{m}$, respectively.

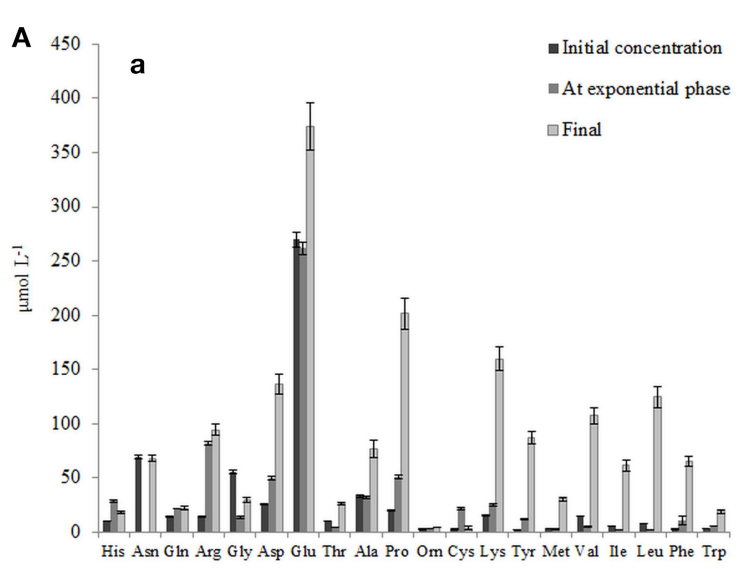

B

a

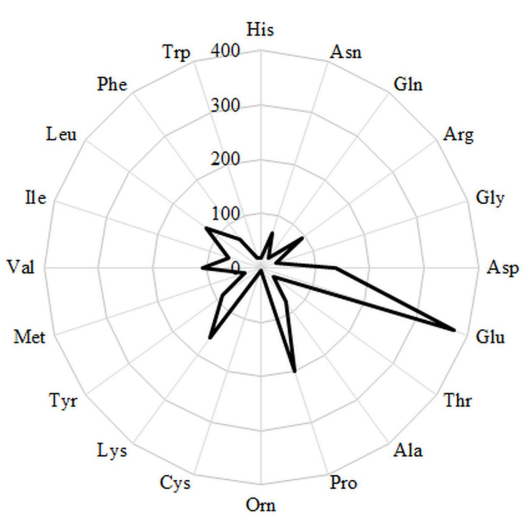

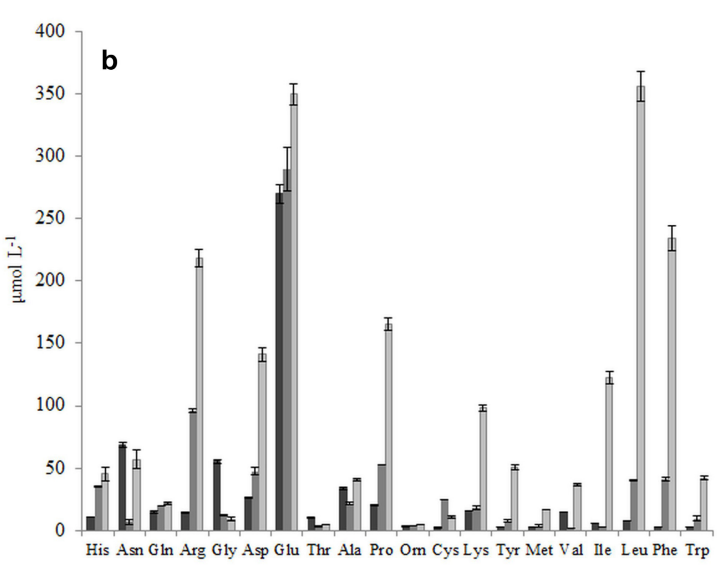

b

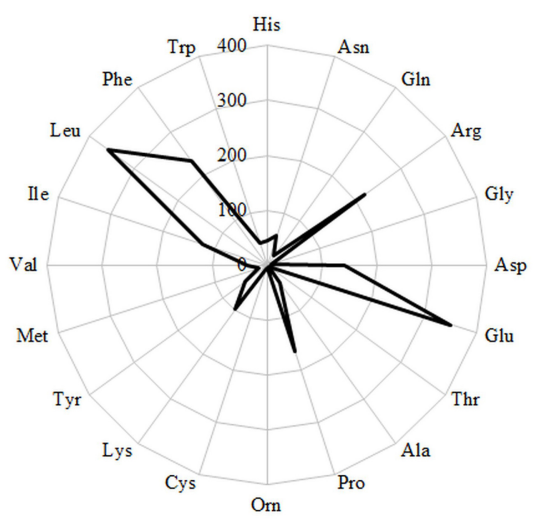

FIGURE 7 | (A) Initial concentrations of free amino acid (FAA) and concentrations of FAA at the end of the exponential phase and at the end of growth in RSM (a) and r-RSM(b). (B) Radar-diagram showing the concentrations of FAA at the end of growth (at $22 \mathrm{~h}$ ) in RSM (a) and r-RSM (b). 
It should be emphasized that only one-third of biomass was synthesized by the end of the second exponential growth phase in r-RSM whereas most of the biomass was synthesized during the post-exponential growth phase (Table 3 ). In contrast to that radii of the colonies at the ends of the second exponential phases reached approximately $70 \%$ of the final values.

If the data obtained showed that the mechanisms determining the end of the exponential phase and end of cultivation in our experiments at $22 \mathrm{~h}$ were not depending on the inoculation rates, then the mechanism leading to the "deviation moment" is more complicated. As seen from the Tables 3, 4 neither the total numbers of bacteria $\mathrm{N}^{*}$ nor the sizes of average colonies were equal at different inoculation rates at the moment when the growth rate of bacteria decreased in the r-RSM in comparison with that in RSM.

\section{UTILIZATION OF CARBOHYDRATES AND PRODUCTION OF LACTIC ACID BY THE BACTERIA IN r-RSM}

The studies of consumption of lactose and formation of organic acids were carried out in the case of inoculation rate of $10^{5} \mathrm{CFU}$ $\mathrm{mL}^{-1}$.

The changes of the concentration of carbohydrates (lactose, glucose, galactose) and lactic acid during fermentation of RSM and r-RSM with St. thermophilus ST12 at inoculation rate $10^{5}$ $\mathrm{CFU} \mathrm{mL} \mathrm{m}^{-1}$ are presented in Figure 4. As seen from this figure, the patterns of hydrolysis of lactose, release of glucose and galactose and formation of lactic acid were practically identical in both RSM and r-RSM (Figure 4). Approximately $130 \mathrm{mmol} \mathrm{L}^{-1}$ of lactose was hydrolysed at the end of the second exponential growth phase (during active growth), $127 \mathrm{mmol} \mathrm{L}^{-1}$ of glucose and $58 \mathrm{mmol} \mathrm{L}^{-1}$ of galactose was utilized by the bacteria, and about $20 \mathrm{mmol} \mathrm{L}^{-1}$ of lactic acid was produced.

Lactose consumption and lactate production per biomass formed $\left(\mathrm{mmol} \mathrm{gdw}^{-1}\right)$ were calculated (see Section Calculation of Growth Parameters Characterizing Metabolism of the Growing Cells) and are presented in Figures 5A,B. During the growth of St. thermophilus ST12, lactose was consumed very fast-up to 2200 and $1800 \mathrm{mmol} \mathrm{gdw}^{-1}$ in liquid and renneted RSM, respectively. Lactose consumption led to the accumulation of galactose, as well as glucose (Figure 5), due to low glycolytic activity of the bacteria, reflected by the low lactate yield per lactose below $0.2 \mathrm{~mol} \mathrm{~mol}^{-1}$ in RSM as well as in r-RSM (theoretical maximum $4 \mathrm{~mol} \mathrm{~mol}^{-1}$ ). It can be assumed that galactose was not metabolized by this strain; however, the accumulation of galactose was lower than predicted compared to the consumption of lactose. This indicated that either galactose was

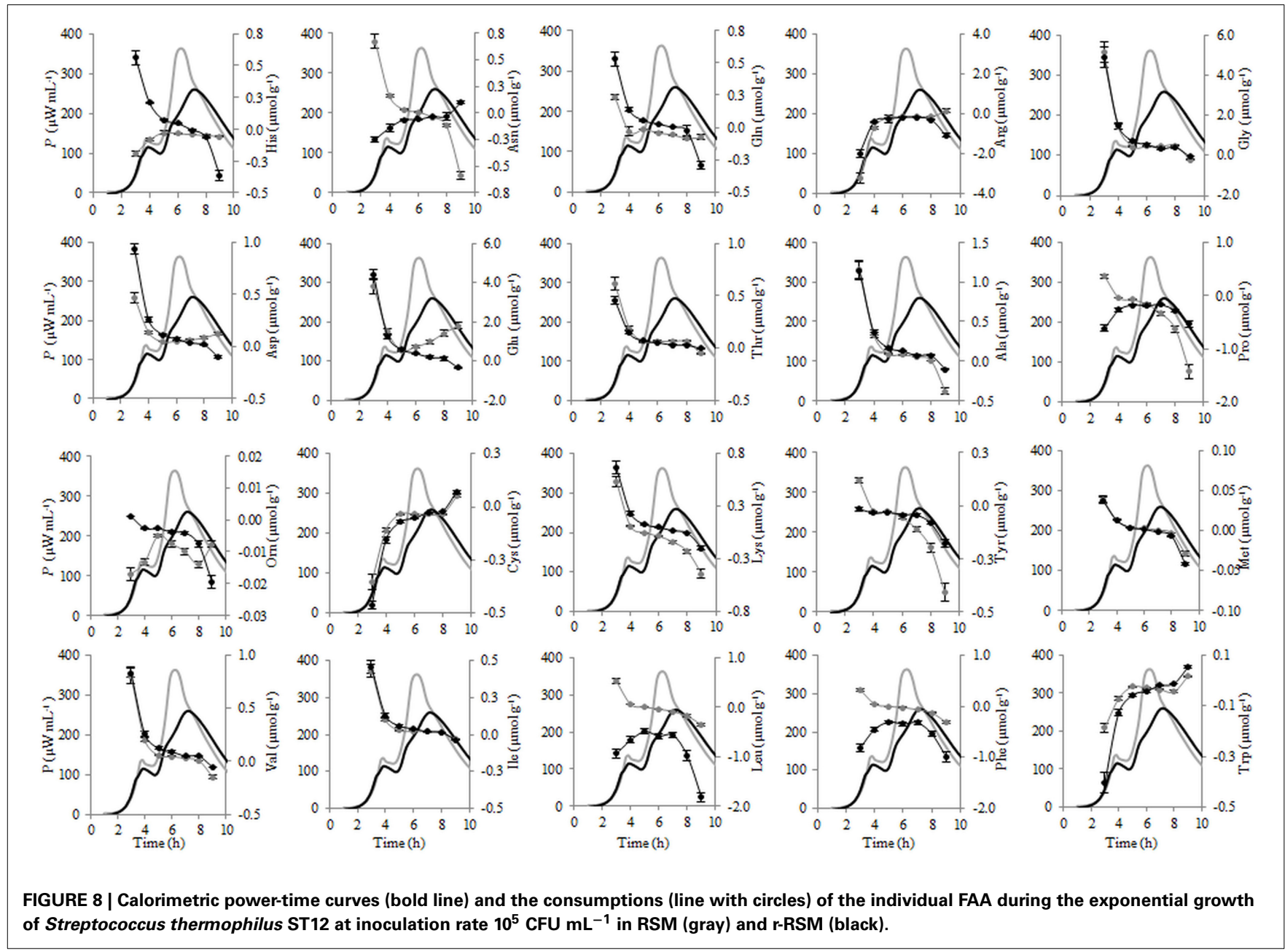


consumed through glycolysis, or it was used for polysaccharide production. Usually polysaccharides are produced in growthrestricted conditions to synthesize carbon storage substances for the future.

\section{CHANGE OF FREE AMINO ACIDS DURING FERMENTATION IN r-RSM}

The changes of the concentrations of the individual FAA during fermentation of liquid and renneted RSM with St. thermophilus ST12 at inoculation $10^{5} \mathrm{CFU} \mathrm{mL}{ }^{-1}$ were evaluated and presented in Figure 6. As seen from Figure 6, glutamic acid was initially the most abundant free amino acid in both, RSM and r-RSM, accounting for $46 \%$ of the total.

There was a dramatic reduction of asparagine, glycine and valine at the end of exponential phase in both RSM and r-RSM. Leucine showed a clear decline in RSM during fermentation, whereas in the case of r-RSM a larger increase of this amino acid as well as phenylalanine was observed. It can be seen from the data presented in Figure 7 that the intensive liberation of the majority of amino acids in RSM and r-RSM was observed during the period from the end of exponential phase till the end of fermentation (from $5 \mathrm{~h}$ till $22 \mathrm{~h}$ ).

The concentrations of the total free amino acids (TFAA) were $15 \%$ higher in r-RSM than those determined in RSM at the end of cultivations, 2024 and $1713 \mu \mathrm{mol} \mathrm{L}^{-1}$, respectively. Glutamic acid, proline, lysine and aspartic acid were the most predominant FAA in RSM at the end of fermentation (at $22 \mathrm{~h}$ ), accounting for $22,12,9$, and $8 \%$ of the total, respectively. In contrast, leucine, glutamic acid, phenylalanine, arginine and proline, accounting for $18,17,12,11$, and $8 \%$ of the total were the dominant amino acids in r-RSM-see Figure 8.

\section{METABOLISM OF AMINO ACIDS AND GROWTH OF THE BACTERIA}

Proteolysis in rennet curd is catalyzed by enzymes from coagulant and enzymes from the inoculated St. thermophilus The initial hydrolysis of caseins is caused by the chymosin which results in the formation of large (water-insoluble) and intermediate-sized (water-soluble) peptides which are degraded subsequently by the coagulant and enzymes from the starters (Sousa et al., 2001). The final products of proteolysis are FAA and their concentration in curd is the net result of the liberation of amino acids from casein, their degradation to catabolic products and also utilization and synthesis by the starter bacteria. Free amino acid concentrations changes were evaluated in liquid and renneted milk samples during the growth of St. thermophilus ST12-see Figures 6-8. Amino acid consumption during the 1 st and 2 nd exponential growth phases is presented in Table 5 Although St. thermophilus has not been shown to be auxotrophic for any single amino acid, growth is abolished when both Glu/Gln and Cys/Met are removed (Letort et al., 2002). In addition, the exhaustion of Leu and Val reduces the growth rate more than three times and only six amino acids (Asn, Ala, Ile, Gly, Ser, and Thr) can be individually omitted without any effect on the growth rate.

Most of the trends in consumption of FAA during the exponential growth of St. thermophilus ST12 in RSM and r-RSM were similar-see Tables 5, 6. The only amino acids consumed in amounts corresponding to the calculated requirements for

Table 5 | Relative consumption of free amino acids (r-I_FAA) compared to that required for the synthesis of biomass proteins (\%).

\begin{tabular}{|c|c|c|c|c|c|c|c|c|c|c|c|c|c|}
\hline \multicolumn{2}{|c|}{ Time of growth, $h$} & 3 & 4 & 5 & 6 & 7 & 8 & 3 & 4 & 5 & 6 & 7 & 8 \\
\hline \multicolumn{2}{|r|}{$\mu$} & 1.71 & 0.90 & 0.59 & 0.77 & 0.46 & 0.24 & 1.68 & 0.88 & 0.47 & 0.47 & 0.52 & 0.43 \\
\hline $\begin{array}{l}\text { r-I_FAA } \\
\text { mmol g }^{-1}\end{array}$ & $\begin{array}{c}\text { Content in } \\
\text { biomass, } \mathrm{mmol} \mathrm{g}^{-1}\end{array}$ & \multicolumn{6}{|c|}{ RSM } & \multicolumn{6}{|c|}{ r-RSM } \\
\hline r-I_Asn & 0.22 & 2014 & 521 & 305 & 82 & -1 & -9 & 402 & 555 & 231 & 119 & -22 & -24 \\
\hline r-I_GIn & 0.19 & 61 & -23 & -104 & -33 & -4 & 8 & 3 & -36 & -111 & -43 & 2 & 8 \\
\hline r-I_Asp & 0.22 & -249 & -85 & -89 & -125 & -107 & -134 & -38 & 2 & 31 & -65 & -148 & -128 \\
\hline r-I_Glu & 0.19 & -302 & 133 & 375 & 70 & -49 & -108 & 69 & 163 & 13 & -182 & -241 & -205 \\
\hline r-I_Thr & 0.30 & 9 & 27 & 51 & 23 & 6 & -2 & 9 & 33 & 40 & 50 & 19 & 8 \\
\hline r-I_Ala & 0.64 & -11 & 4 & 11 & 4 & 0 & -2 & 5 & 26 & 38 & 42 & 12 & 1 \\
\hline r-I_Pro & 0.18 & -498 & -216 & -241 & -160 & -102 & -121 & -76 & -162 & -200 & -244 & -140 & -85 \\
\hline r-I_Val & 0.36 & 109 & 49 & 64 & 4 & -19 & -32 & 26 & 70 & 79 & 59 & -7 & -19 \\
\hline r-I_Ile & 0.27 & 105 & 36 & 26 & 2 & -8 & -14 & 31 & 53 & 22 & -1 & -19 & -15 \\
\hline r-I_Leu & 0.39 & 122 & 32 & 19 & -7 & -19 & -30 & 1 & -58 & -125 & -155 & -115 & -81 \\
\hline r-I_Phe & 0.18 & -9 & -36 & -69 & -59 & -38 & -43 & -24 & -191 & -335 & -423 & -236 & -144 \\
\hline r-I_Trp & 0.07 & 34 & -29 & -76 & -54 & -31 & -27 & 10 & -93 & -207 & -234 & -99 & -59 \\
\hline
\end{tabular}

Green color shows that free amino acids were consumed more than required for biomass synthesis. Amino acid composition of biomass is taken from the literature (Adamberg et al., 2012). 
Table 6 | Milk composition, maximal amount of biomass that can be obtained from free amino acids (FAA) in milk and relative amount of FAA supporting the synthesis of biomass proteins.

\begin{tabular}{|c|c|c|c|c|c|c|c|c|c|}
\hline Aminoacid & \multicolumn{2}{|c|}{$\begin{array}{l}\text { Composition of } \\
\text { milk }\left(C_{F A A}\right), m M\end{array}$} & Biom. $\left(B_{A A}\right), \mathrm{mmol} \mathrm{g}^{-1}$ a & \multicolumn{2}{|c|}{$\begin{array}{c}\text { Theoretical amount of } \\
\text { biomass from } \\
\text { FAA (Xtheor), } \mathrm{g} \mathrm{L}^{-1} \mathrm{~b}\end{array}$} & \multicolumn{2}{|c|}{$\begin{array}{l}\text { Experimentally determined } \\
\text { biomass (Xexp) at } 8 \mathrm{~h}, \\
\mathrm{~g} \mathrm{~L}^{-1 \mathrm{c}}\end{array}$} & \multicolumn{2}{|c|}{$\begin{array}{l}\text { Max amount of FAA } \\
\text { (r-I_FAA max), \%d }\end{array}$} \\
\hline His & 10.4 & 10.5 & 0.08 & 0.12 & 0.12 & 0.12 & 0.18 & 100 & 70 \\
\hline Asn & 70.1 & 69.9 & 0.22 & 0.33 & 0.32 & 0.12 & 0.18 & 262 & 181 \\
\hline Gly & 55.7 & 56.0 & 0.45 & 0.12 & 0.12 & 0.12 & 0.18 & 99 & 69 \\
\hline Asp & 26.9 & 26.5 & 0.22 & 0.12 & 0.12 & 0.12 & 0.18 & 101 & 69 \\
\hline Glu & 270.0 & 271.0 & 0.19 & 1.46 & 1.46 & 0.12 & 0.18 & 1176 & 817 \\
\hline Thr & 10.2 & 10.1 & 0.3 & 0.03 & 0.03 & 0.12 & 0.18 & 27 & 19 \\
\hline Ala & 33.6 & 33.6 & 0.64 & 0.05 & 0.05 & 0.12 & 0.18 & 42 & 29 \\
\hline Met & 3.0 & 3.0 & 0.07 & 0.04 & 0.04 & 0.12 & 0.18 & 36 & 25 \\
\hline Val & 15.1 & 14.9 & 0.36 & 0.04 & 0.04 & 0.12 & 0.18 & 33 & 23 \\
\hline Ile & 6.0 & 6.0 & 0.27 & 0.02 & 0.02 & 0.12 & 0.18 & 18 & 12 \\
\hline Leu & 8.3 & 8.1 & 0.39 & 0.02 & 0.02 & 0.12 & 0.18 & 17 & 12 \\
\hline Phe & 3.0 & 2.9 & 0.18 & 0.02 & 0.02 & 0.12 & 0.18 & 13 & 9 \\
\hline Trp & 3.2 & 3.3 & 0.07 & 0.04 & 0.05 & 0.12 & 0.18 & 36 & 26 \\
\hline
\end{tabular}

a Biomass composition is taken from literature (Adamberg et al., 2012).

${ }^{b}$ Calculated as $X_{\text {theor }}=\left(C_{A A} / 1000\right) / B_{A A}$ indicating how much biomass can be produced from free amino a acid present in milk assuming that amino acid is used only for the synthesis of biomass proteins. AA refers to corresponding amino acid.

${ }^{c}$ Experimentally determined biomass concentration assuming that heat produced per cell is $4.45 \times 10^{-9} \mathrm{~J}$ per cell and cell mass is $0.2 \times 10^{-12} \mathrm{~g}$.

${ }^{d}$ Calculated as FI_FAA max $=X_{\text {theor }} \times 100 / X_{\text {exp }}$ indicating relative amount of amino acid in biomass proteins that can be supported by consumed free amino acids. Percentage below indicates that free amino acid was less consumed than required for synthesis of biomass proteins.

biomass synthesis in the 1st exponential phase were Gly, Glx $(\mathrm{Gln}+\mathrm{Glu})$ and Asx. These amino acids were also the only amino acids whose concentrations in milk exceeded the amount required for biomass synthesis (Table 6). The amount of free His was also sufficient to satisfy biomass requirements, but it should be noted that the amount of this amino acid did not decrease during the exponential growth of the bacteria. Most of the concentrations of other FAA could not support the growth of cells more than 30\%. Free Arg and Pro in milk could support amino acid requirements for over $40 \%$ but instead of decrease the concentrations of these amino acids increased during the 1st exponential growth phase. This pattern was quite general. Accumulation of amino acids in the media, especially in renneted milk samples due to their release from proteins, and most noticeably during the second exponential growth phase, made it impossible to analyse consumptions of amino acids in this growth phase.

Overconsumption of Glu 5-7 times exceeding the need for biomass synthesis indicated the potential of the synthesis of other amino acids from it. In total, $4.6 \mathrm{mmol} \mathrm{g} \mathrm{g}^{-1}$ of amino acids were incorporated into the biomass. Of the total, $30 \%$ was covered by the consumption of FAA (excluding Glu). If we take into account Glu overconsumption and amino group transfer to other amino acids, Glu can provide ammonia for $25 \%$ of amino acids of the biomass. The remaining $45 \%$ of amino acids must be have been derived from peptides.

\section{DISCUSSION}

During the last years, there has been a significant increase of interest in study of growth of bacterial colonies in dairy food matrices (especially in cheese matrices). It has been shown that location, distribution of the cells in different zones of the matrices as well as size of the colonies and distances between colonies are important factors determining peculiarities of cheese ripening processes, influencing utilization of the nutrients and diffusion of metabolites within the cheese matrices (Ercolini et al., 2003; Jeanson et al., 2011; Floury et al., 2013). However, the first systematic comparative study of quantitative peculiarities of growth and metabolism of a LAB St. thermophilus ST12 at different inoculation rates in liquid and renneted milk during the whole growth of the bacteria was carried out in this paper to our knowledge. A novel non-invasive method of microcalorimetry developed by us was used throughout the study. The method developed and used allowed obtain the first time quantitative data on the growth kinetics of colonies of different sizes in renneted milk.

It was shown that the growth patterns of St. thermophilus ST12 in r-RSM during fermentation at $40^{\circ} \mathrm{C}$ were clearly multiphase 
in comparison with diauxic growth of St. thermophilus ST12 in RSM. Decrease of the maximal calorimetric growth rates $\mu_{\max }$ during the first and the second exponential growth phases as well as remarkably lower values of maximum heat flows $P_{\max }$ $\left(\mathrm{W} \mathrm{mL} \mathrm{m}^{-1}\right.$ ) of the thermophilic starters growing in r-RSM were observed. The noted inhibition of growth rates in the r-RSM could be explained most probably by the accumulation of lactate in the colonies leading to the change of $\mathrm{pH}$. This conclusion was supported also by the fact that lactate inhibition was not observed in RSM where the buffering capacity of liquid milk was sufficient for alleviating of the influence of lactate (see Figure 3 ).

The data obtained showed that the numbers of bacteria in the end of the exponential phase (combined exponential phase, see above), as well as in the end of the cultivations were practically the same at different inoculation rates, see Table 3. This allowed introduce a very simple calculation scheme for the determination of the sizes of the colonies, including the radii, in these important points at different inoculation rates presented in Table 3. However, the total numbers of bacteria in the end of the exponential growth in r-RSM were higher than in RSM for about $13 \%$ at all except at low inoculation rates. This fact could be explained by the lower rate of decrease of $\mathrm{pH}$ in the r-RSM in comparison with RSM indicating that buffering capacity of the matrices is important in determining the end of the exponential growth of the bacteria in milk, not accumulation of lactate directly.

It should be noted that the total numbers of bacteria in the "deviation points" were not the same at different inoculation rates as expected from the data presented above. This was indicating that these points were determined not by a "uniform" mechanism like the end of exponential growth phases and the end of growth during the cultivation but in this case most probably also the sizes of colonies were playing more important role in determining the local buffering capacity in the colonies. Another factor derived from the size of colonies is the availability of nutrients (essential amino acids) for bacteria as proteases are cell bound and cannot freely move in the environment to deliberate peptides from casein.

An observation showing that the growth rates of bacteria in small colonies in the beginning of the cultivation at all inoculation rates were the same as in liquid milk indicated that diffusion rate of glucose was practically the same in these two types of samples.

\section{CONCLUSIONS}

The present study clearly showed potential of non-invasive method of microcalorimetry in combination with the other analytical methods in study of quantitative peculiarities of growth and metabolism of bacteria in opaque and solid state media-see a detailed comparison of the methods in Lobete et al. (in press). It was possible to elucidate the quantitatively characterized growth patterns of Streptococcus thermophilus ST12 in renneted milk at different inoculation rates and compare them with the patterns of growth of the bacteria in milk. Clear discriminative results of growth and metabolic patterns of cells in renneted and liquid milk encourage use of microcalorimetry more widely as a screening tool for strain selection in their natural environment (milk or curd).

\section{ACKNOWLEDGMENTS}

The financial support for this research was financially supported through the grant ETF7636 of Estonian Science Foundation, through the institutional research funding (IUT 1927) of Estonian Ministry of Education and Research, and by the EU Regional Development Fund projects EU28912, EU29994 and 3.2.0701.110018.

\section{REFERENCES}

Adamberg, K., Seiman, A., and Vilu, R. (2012). Increased biomass yield of Lactococcus lactis by reduced overconsumption of amino acids and increased catalytic activities of enzymes. PLOS ONE 7:e48223. doi: 10.1371/journal.pone.0048223

Berridge, N. J., Cousins, C. M., and Cliffe, A. J. (1974). Microcalorimetry applied to certain species of bacteria growing in sterilized separated milk. J. Dairy Res. 41, 203-215. doi: 10.1017/S0022029900019622

Brocklehurst, T. F., Mitchell, G. A., Ridge, Y. P., Seale, R., and Smith, A. C. (1995). The effect of transient temperatures on the growth of Salmonella typhimurium LT2 in gelatin gel. Int. J. Food Microbiol. 27, 45-60. doi: 10.1016/01681605(94)00152-V

Ercolini, D., Hill, P. J., Dodd, C. E. R. (2003). Bacterial community structure and location in Stilton cheese. Appl. Environ. Microbiol. 69, 3540-3548. doi: 10.1016/j.ijfoodmicro.2006.09.003

Favrot, C., and Maubois, J. L. (1994). Growth of Lactococcus lactis subsp lactis in reconstituted irradiated milk powder. Lait 74, 253-266. doi: 10.1051/lait:1994421

Favrot, C., and Maubois, J. L. (1996). Growth of Lactococcus lactis in milk and rennet curd: influence of the level of inoculation. Lait 76, 193-208. doi: 10.1051/lait:1996317

Floury, J., Jeanson, S., Aly, S., and Lortal, S. (2010). Determination of the diffusion coefficients of small solutes in cheese: a review. Dairy Sci. Technol. 90, 477-508. doi: $10.1051 / \mathrm{dst} / 2010011$

Floury, J., Jeanson, S., Madec, M.-N., and Lortal, S. (2013). Porosity of Lactococcus lactis subsp. lactis LD61 colonies in model cheese. Int. J. Food Microbiol. 163, 64-70. doi: 10.1016/j.ijfoodmicro.2013.02.014

Fujita, T., Monk, P. R., and Wadsö, I. (1978). Calorimetric identification of several strains of lactic acid bacteria. J. Dairy Res. 45, 457-463.

Gardea, A. A., Carvajal-Millán, E., Higuera-Ciapara, I., Figueroa, C., MolinaCorral, J., Rascón, A., et al. (2002). Calorimetric assessment of microbial growth in milk as affected by different conditions. Thermochim. Acta 394, 179-184. doi: 10.1016/S0040-6031(02)00248-4

Hales, T. C. (1994). The status of the Kepler conjecture. Math. Intell. 16, 47-58.

Hsiang, W.-I. (1993). On the sphere packing problem and the proof of Kepler's conjecture. Int. J. Math. 4, 739-831.

Jeanson, S., Chadœuf, J., Madec, M. N., Aly, S., Floury, J., Brocklehurst, T. F., et al. (2011). Spatial distribution of bacterial colonies in a model cheese. Appl. Environ. Microbiol. 77, 1493-1500. doi: 10.1128/AEM.02233-10

Kabanova, N., Kazarjan, A., Stulova, I., and Vilu, R. (2009). Microcalorimetric study of growth of Lactococcus lactis IL1403 at different glucose concentrations in broth. Thermochim. Acta 496, 87-92. doi: 10.1016/j.tca.2009.07.003

Kabanova, N., Stulova, I., and Vilu, R. (2012). Microcalorimetric study of the growth of bacterial colonies of Lactococcus lactis IL1403 in agar gels. Food Microbiol. 29, 67-79. doi: 10.1016/j.fm.2011.08.018

Kriščiunaite, T., Stulova, I., Kabanova, N., Laht, T.-M., and Vilu, R. (2011). The effect of hydrogen peroxide on the growth of thermophilic lactic starter and acid gelation of UHT milk. Int. Dairy J. 21, 239-246. doi: 10.1016/j.idairyj.2010.11.012

Letort, C., Nardi, M., Garault, P., Monnet, V., and Juillard, V. (2002). Casein utilization by Streptococcus thermophilus results in a diauxic growth in milk. Appl. Environ. Microbiol. 68, 3162-3165. doi: 10.1128/AEM.68.6.31623165.2002

Lobete, M. M., Fernandes, E. N., and Van Impe, J. F. M. (in press). Recent trends in non-invasive in situ techniques to monitor bacterial colonies in solid (model) food. Front. Microbiol.

Malakar, P. K., Martens, D. E., van Breukelen, W., Boom, R. M., Zwietering, M. H., and van't Riet, K. (2002). Modeling the interactions of Lactobacillus curvatus colonies in solid medium: consequences for food quality and 
safety. Appl. Environ. Microbiol. 68, 3432-3441. doi: 10.1128/AEM.68.7.34323441.2002

Maskow, T., and Babel, W. (2003). Thermokinetic description of anaerobic growth of Halomonas halodenitrificans using a static microcalorimetric ampoule technique. J. Biotech. 101, 267-274. doi: 10.1016/S0168-1656(02) 00341-3

McKay, A. L., Peters, A. C., and Wimpenny, J. W. T. (1997). Determining specific growth rates in different regions of Salmonella typhimurium colonies. Lett. Appl. Microbiol. 24, 74-76. doi: 10.1046/j.1472-765X.1997.00354.x

Monk, P. R. (1979). Thermograms of Streptococcus thermophilus and Lactobacillus bulgaricus in single and mixed culture in milk medium. J. Dairy Res. 46, 485-496. doi: 10.1017/S0022029900017519

Riva, M., Franzetti, L., Galli, A., and Schiraldi, A. (1997). Growth and fermentation activity of Streptococcus thermophilus and Lactobacillus delbrueckii subsp. bulgaricus in milk: a calorimetric investigation. Ann. Microbiol. Enzimol. 47, 199-211.

Sousa, M. J., Ardö, Y., and McSweeny, P. L. H. (2001). Advances in the study of proteolysis during cheese ripening. Int. Dairy J. 11, 327-345. doi: 10.1016/S09586946(01)00062-0

Stulova, I., Kabanova, N., Kriščiunaite, T., Laht, T.-M., and Vilu, R. (2011). The effect of milk heat treatment on the growth characteristics of lactic acid bacteria. Agron. Res. 9, 473-478.

Stulova, I., Kabanova, N., Kriščiunaite, T., Taivosalo, A., Laht, T.-M., and Vilu, R. (2013). Fermentation of reconstituted milk by Streptococcus thermophilus: effect of irradiation on skim milk powder. Int. Dairy J. 31, 139-149. doi: 10.1016/j.idairyj.2013.02.004

Velliou, E. G., Noriega, E., Van Derlinden, E., Mertens, L., Boons, K., Geeraerd, A. H., et al. (2013). The effect of colony formation on the heat inactivation dynamics of Escherichia coli K12 and Samonella typhimurium. Food Res. Int. 54, 1746-1752. doi: 10.1016/j.foodres.2013.09.009

Wadsö, L., and Galindo, F. G. (2009). Isothermal calorimetry for biological applications in food science and technology. Food Control 20, 956-961. doi: 10.1016/j.foodcont.2008.11.008

Wilson, P. D. G., Brocklehurst, T. F., Arino, S., Thuault, D., Jakobsen, M., Lange, M., et al. (2002). Modelling microbial growth in structured foods: towards a unified approach. Int. J. Food Microbiol. 73, 275-289. doi: 10.1016/S01681605(01)00660-2

Conflict of Interest Statement: The authors declare that the research was conducted in the absence of any commercial or financial relationships that could be construed as a potential conflict of interest.

Received: 06 October 2014; accepted: 22 January 2015; published online: 10 February 2015.

Citation: Stulova I, Kabanova N, Kriščiunaite T, Adamberg K, Laht T-M and Vilu $R$ (2015) Microcalorimetric study of the growth of Streptococcus thermophilus in renneted milk. Front. Microbiol. 6:79. doi: 10.3389/fmicb.2015.00079

This article was submitted to Food Microbiology, a section of the journal Frontiers in Microbiology.

Copyright (ㅇ) 2015 Stulova, Kabanova, Kriščiunaite, Adamberg, Laht and Vilu. This is an open-access article distributed under the terms of the Creative Commons Attribution License (CC BY). The use, distribution or reproduction in other forums is permitted, provided the original author(s) or licensor are credited and that the original publication in this journal is cited, in accordance with accepted academic practice. No use, distribution or reproduction is permitted which does not comply with these terms. 


\section{Measurement of $\mathrm{pH}$ micro-heterogeneity in natural cheese matrices by fluorescence lifetime imaging}

OPEN ACCESS

Edited by:

Sophie Jeanson,

Institut National de la Recherche

Agronomique, France

Reviewed by:

Carmen Wacher

Universidad Nacional Autónoma de

México, Mexico

Christelle Lopez,

Institut National de la Recherche

Agronomique, France

*Correspondence:

Zuzana Burdikova,

Teagasc Food Research Centre

Moorepark, Fermoy, Co. Cork, Ireland

zuzana.burdikova@teagasc.ie

Specialty section:

This article was submitted to Food Microbiology, a section of the journal

Frontiers in Microbiology

Received: 28 September 2014

Accepted: 19 February 2015

Published: 06 March 2015

Citation:

Burdikova Z, Svindrych Z, Pala J,

Hickey CD, Wikinson MG, Panek J, Auty MAE, Periasamy A and Sheehan

JJ (2015) Measurement of $\mathrm{pH}$

micro-heterogeneity in natural cheese

matrices by fluorescence lifetime

imaging. Front. Microbiol. 6:183.

doi: 10.3389/fmicb.2015.00183

\author{
Zuzana Burdikova ${ }^{1 *}, Z_{\text {denek Svindrych }}^{2}$, Jan Pala ${ }^{3}$, Cian D. Hickey ${ }^{1}$, \\ Martin G. Wilkinson ${ }^{4}$, Jiri Panek ${ }^{5}$, Mark A. E. Auty ${ }^{1}$, Ammasi Periasamy ${ }^{2}$ and \\ Jeremiah J. Sheehan ${ }^{1}$
}

${ }^{1}$ Teagasc Food Research Centre, Moorepark, Fermoy, Ireland, ${ }^{2}$ Department of Biology, W. M. Keck Center for Cellular Imaging, University of Virginia, Charlottesville, VA, USA, ${ }^{3}$ Department of Sport Medicine, Third Faculty of Medicine, Charles University, Prague, Czech Republic, ${ }^{4}$ Department of Life Sciences, Faculty of Science and Engineering, University of Limerick, Limerick, Ireland, ${ }^{5}$ Institute of Macromolecular Chemistry, Academy of Sciences of the Czech Republic, Prague,

Czech Republic

Cheese, a product of microbial fermentation may be defined as a protein matrix entrapping fat, moisture, minerals and solutes as well as dispersed bacterial colonies. The growth and physiology of bacterial cells in these colonies may be influenced by the microenvironment around the colony, or alternatively the cells within the colony may modify the microenvironment (e.g., pH, redox potential) due to their metabolic activity. While cheese $\mathrm{pH}$ may be measured at macro level there remains a significant knowledge gap relating to the degree of micro-heterogeneity of $\mathrm{pH}$ within the cheese matrix and its relationship with microbial, enzymatic and physiochemical parameters and ultimately with cheese quality, consistency and ripening patterns. The $\mathrm{pH}$ of cheese samples was monitored both at macroscopic scale and at microscopic scale, using a non-destructive microscopic technique employing C-SNARF-4 and Oregon Green 488 fluorescent probes. The objectives of this work were to evaluate the suitability of these dyes for microscale $\mathrm{pH}$ measurements in natural cheese matrices and to enhance the sensitivity and extend the useful $\mathrm{pH}$ range of these probes using fluorescence lifetime imaging (FLIM). In particular, fluorescence lifetime of Oregon Green 488 proved to be sensitive probe to map $\mathrm{pH}$ micro heterogeneity within cheese matrices. Good agreement was observed between macroscopic scale $\mathrm{pH}$ measurement by FLIM and by traditional $\mathrm{pH}$ methods, but in addition considerable localized microheterogeneity in $\mathrm{pH}$ was evident within the curd matrix with $\mathrm{pH}$ range between 4.0 and 5.5. This technique provides significant potential to further investigate the relationship between cheese matrix physico-chemistry and bacterial metabolism during cheese manufacture and ripening.

Keywords: cheese matrix, C-SNARF-4, Oregon Green, pH micro-heterogeneity, fluorescence lifetime, FLIM, natural cheese 


\section{Introduction}

Cheese is a complex physiochemical and microbial system containing many interacting components. It may be defined as a protein matrix entrapping fat, moisture, minerals and other solutes as well as dispersed bacterial colonies. Irrespective of cheese type, both starter and non-starter bacteria are immobilized and isolated within the curd matrix during the coagulation step of cheese manufacture (Fox et al., 2000; McSweeney, 2004b; Jeanson et al., 2011). Each inoculated bacterial cell is assumed to grow, generating colonies which are dispersed within the cheese curd. Bacterial colonies within the cheese matrix have been shown to be located on or within milk fat globular membrane material (MFGM) and at fat-protein interfaces with the curd (Laloy et al., 1996; Lopez et al., 2006) and are considered to interact with the cheese matrix during ripening (Feeney et al., 2002; McSweeney, 2004a). The growth and physiology of bacterial cells in these colonies may be influenced by the microenvironment around the colony, or alternatively the cells within the colony may modify the microenvironment (e.g., $\mathrm{pH}$, redox potential) due to their metabolic activity (McSweeney, 2004a).

The $\mathrm{pH}$ of cheese curd is determined by the extent of acidification during manufacture, by the availability of substrate for fermentation, principally lactose, by the buffering capacity of the cheese curd and, in some cases, by the degree of deacidification during ripening. Cheese $\mathrm{pH}$ affects the texture of curd directly by influencing the degree of casein hydration, which in turn influences the visco-elastic behavior of the protein matrix (Euston et al., 2002; Kilcast and Angus, 2007). pH also affects texture and flavor indirectly by influencing the activity of enzymes important to ripening, e.g., plasmin (Grufferty and Fox, 1988) and, in the case of the coagulant, both the retention of and the activity of the enzyme in the curd during manufacture and subsequent ripening (Holmes et al., 1977; Stadhouders et al., 1977; Visser, 1977; Creamer et al., 1985; Garnot et al., 1987). The pH also influences the metabolic activity of lactic acid bacteria (Meldrum et al., 2003; Kajfasz and Quivey, 2011; Jeanson et al., 2013). The pH is a key factor influencing amino acid decarboxylase activity (Gardini et al., 2001) and bacteriocin production (Foulquié Moreno et al., 2003).

Development of a method capable of precisely measuring $\mathrm{pH}$ at localized level within the cheese matrix will facilitate a new understanding of the relationship between cheese matrix physico-chemistry and bacterial metabolism during cheese manufacture and ripening.

Until now different techniques have been used to monitor $\mathrm{pH}$ in and around colonies. Microelectrodes were first used to measure $\mathrm{pH}$ in and around submerged colonies of S. typhimurium (Wimpenny et al., 1995; Jeanson et al., 2013), however, these cannot map the spatial variation of $\mathrm{pH}$ to a resolution of a micrometer. Standard methodologies to measure the $\mathrm{pH}$ in cell biology include the use of $\mathrm{pH}$ sensitive fluorescent dyes-fluorescein based $\mathrm{pH}$ indicator, benzoxanthene dyes, cyanine-based $\mathrm{pH}$ indicators, etc. (Han and Burgess, 2010). In the vast majority of these experiments Confocal Laser Scanning Fluorescence Microscopy (CLSM) is employed, mainly due to its ability to perform optical sectioning and provide accurate three-dimensional representation of the fluorophore distribution (Inoue, 1995).

Seminaphthorhodafluor-4F 5-(and-6) carboxylic acid (C-SNARF-4) is a long wavelength fluorescent $\mathrm{pH}$ indicator (Haugland, 2002). The dye exhibits two emission peaks whose intensities display different $\mathrm{pH}$ dependencies, thus it is suitable as a ratiometric fluorescent $\mathrm{pH}$ probe. The results of the fluorescence intensity ratio of these two peaks have been confirmed as a reliable indicator of $\mathrm{pH}$ at values 5.0 and above (Hunter and Beveridge, 2005). However, in more acidic environments the $\mathrm{pH}$ sensitivity of the fluorescence ratio decreases rapidly.

Oregon Green 488 is a fluorinated analog of fluorescein. It exhibits higher photostability and lower $\mathrm{pK}_{\mathrm{a}}\left(\mathrm{pK}_{\mathrm{a}}=4.7\right.$ vs. 6.4 for fluorescein), making it a useful $\mathrm{pH}$ indicator in the weakly acidic range ( $\mathrm{pH} 4-6)$. However, to use Oregon Green as a ratiometric $\mathrm{pH}$ probe, two excitation wavelengths are necessary (Whitaker et al., 1991). Oregon Green carboxylic acid has been widely employed to measure lysosomal and endosomal $\mathrm{pH}$ (Dunn et al., 1991). Bioconjugate prepared from Oregon Green 488 , dextran, has the advantage of high stability and low affinity to cheese matrix components (proteins).

Besides fluorescence intensity, fluorescence lifetime, a probabilistic timescale of fluorescence emission (Sun et al., 2011), may provide additional information about the chemical environment (e.g., $\mathrm{pH}$ ) of the probe. The most common example is fluorescein, whose fluorescence lifetime changes from 4 ns at $\mathrm{pH} 10$ to $2 \mathrm{~ns}$ at $\mathrm{pH} 7$ (Lakowicz, 2006), and many other fluorophores were probed for lifetime-pH dependence, including some of the SNARF family (Szmacinski and Lakowicz, 1993). Oregon Green dyes were also probed for lifetime- $\mathrm{Ca}^{2+}$ sensitivity (Agronskaia et al., 2004).

\section{Fluorescence Lifetime Imaging (FLIM)}

Fluorescence of a fluorophore is characterized by its absorption spectrum (a probability that a photon of given wavelength is absorbed by the molecule), quantum yield (a probability that the excited molecule emits a photon during transition to the ground state), its emission spectrum (a distribution of wavelengths of the emitted photon) and the fluorescence lifetime (a characteristic time the molecule spends in the excited state before emitting a photon). Both excitation and emission spectrum are used extensively to distinguish different fluorophores and to sense the local environment of a fluorophore (local ion concentration, electric potential, $\mathrm{pH}$, etc., Lakowicz, 2006). However, only a limited number of fluorophores, often specifically designed, display useful changes in excitation and emission spectra. Here we note that changes in fluorescence intensity alone cannot be exploited in most cases, because the fluorescence intensity depends predominantly on the concentration of the fluorescent molecules. When such fluorophores are not available or do not perform optimally in specific experimental conditions, one may explore the dependence of fluorescence lifetime on the local environment.

The fluorescence lifetime has only recently been widely adopted, mainly because of the technical challenges associated with the measurement of such fast phenomena (typically in the nanosecond range). One of the most efficient precise and 
reliable methods used in conjunction with confocal microscopy is Time Correlated Single Photon Counting (TCSPC). This method relies on sub-nanosecond pulsed illumination and fast photoncounting detectors to measure the delay between the excitation pulse and the photon detection event (Becker, 2005). The intensity of fluorescence following a short excitation pulse (usually much shorter than $1 \mathrm{~ns}$ ) decays exponentially on a nanosecond timescale (Figure 1A). This decay, however, cannot be digitized directly with current instrumentation; moreover, in a typical confocal microscopy experiment there is less than one photon detected in a hundred of laser pulses. Instead, the time delay between the laser pulse and photon detection event is measured (Figure 1B) for each photon and a histogram (Figure 1C) is built from these delays in each pixel of a confocal image. By fitting an exponential model (Equation 1) to these decays, the information about the lifetime of the constituents is recovered.

In simple cases (single molecular species with single ground and excited state configuration, or with configurations changing in sub-nanosecond timescale) single exponential decay completely describes the experimental data. However, often more exponential components are needed to achieve acceptable fit between the model (Equation 1) and the recorded decay. Although the detailed mechanism is often poorly understood, multi-exponential decay suggest the existence of more distinct fluorescent species or several stable configurations of the fluorophore on long timescales (relatively to the fluorescence lifetime). Despite the inherent difficulties and numerical instability of multi-exponential fitting, when performed cautiously, the individual lifetime components can provide valuable information regarding local environment of the fluorophore (Becker, 2005).

The objectives of this study were (i) to assess the potential of C-SNARF-4 and Oregon Green 488 fluorescent dyes to perform as ratiometric $\mathrm{pH}$ indicators in cheese matrices at microscale, (ii) to extend the useful $\mathrm{pH}$ range of the probes using lifetime measurements, and (iii) to determine the level of $\mathrm{pH}$ microheterogeneity within a natural cheese matrix. To our knowledge it is the first time that advanced microscopic technique fluorescent lifetime imaging microscopy (FLIM) of C-SNARF-4 and Oregon Green 488 has been applied to determine localized $\mathrm{pH}$ of different types of natural cheese.

\section{Materials And Methods}

\section{Cheese Preparation And Characterization Starter Strains}

Thermophilic starter cultures typically used in Swiss-type cheese manufacture (Scott, 1981), i.e., S. thermophilus TH3 (ST) and L. helveticus LHB02 (LH) were purchased from Chr. Hansen Ltd. (Little Island, Co. Cork, Ireland) as individual frozen concentrates and stored at $-80^{\circ} \mathrm{C}$ until cheese manufacture.

\section{Cheese Manufacture}

Two cheese making trials consisting of four vats of $454 \mathrm{~kg}$ cheese milk were undertaken over a 6 month period. Raw milk was obtained from a local dairy company, standardized to a protein to fat ratio of $1.01: 1$, held overnight at $4^{\circ} \mathrm{C}$, pasteurized at $72^{\circ} \mathrm{C}$ for $15 \mathrm{~s}$, and pumped at $32^{\circ} \mathrm{C}$ into cylindrical, jacketed, stainless steel vats $(500 \cdot 1)$ with automated variable speed cutting and stirring equipment (APV) Schweiz AG, Worb, Switzerland). Cheesemilk was heated to $34^{\circ} \mathrm{C}$ and inoculated with $0.1 \mathrm{~g} / 1 \mathrm{ST}\left(10^{6} \mathrm{cfu} / \mathrm{ml}\right)$ and $0.05 \mathrm{~g} / \mathrm{LH}\left(5 \cdot 10^{5} \mathrm{cfu} / \mathrm{ml}\right)$. After a $60 \mathrm{~min}$ ripening period, chymosin (Chymax plus, Chr. Hansens Ltd.), diluted 1:6 with deionized water, was added at a level of $18 \mathrm{ml}$ per $100 \mathrm{~kg}$ milk. A coagulation time of $35 \mathrm{~min}$ was allowed prior to the cutting of the coagulum. After a $10 \mathrm{~min}$ healing period, the curd/whey mixture was cooked by steam injection into the jacket of the vat with constant stirring. Maximum scalds employed were 50 or $40^{\circ} \mathrm{C}$
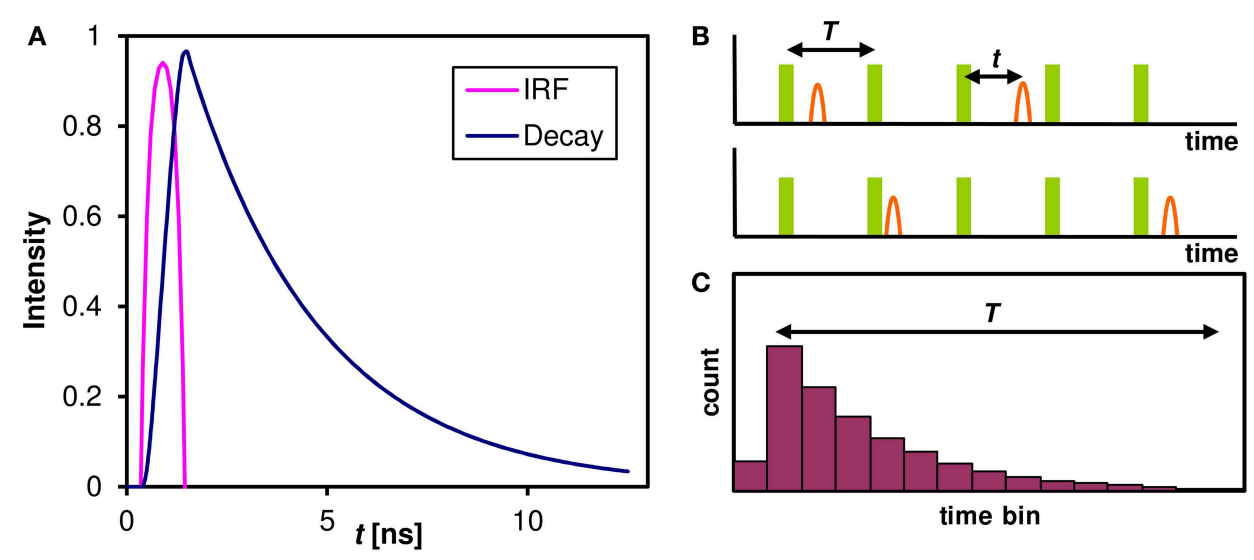

FIGURE 1 | The Fluorescence decay and its measurement. (A) The fluorescence decay of an ensemble of identical molecules after a laser pulse. The Instrumental Response Function (IRF) combines the excitation pulse duration and the time resolution of the detector and an associated hardware. The intensity of a single fluorescent species that can only assume single ground and excited state follows an exponential decay with a characteristic lifetime $\tau$. (B) In a practical TCSPC experiment there is typically much less than one photon detected for every laser pulse. The time of photon arrival $t$ within the laser pulse period $T$ is measured for every photon. In our case of $80 \mathrm{MHz}$ laser pulse repetition rate the period $T=12.5 \mathrm{~ns}$. (C) A histogram of photon arrival times that is built from the single photon events approaches the theoretical ensemble decay curve of (A). This histogram can be built in each pixel of a confocal image and the lifetime information at each pixel is then extracted by fitting an exponential model (Equation 1). 
TABLE 1 | Manufacture parameters used during cheese making trials.

\begin{tabular}{|c|c|c|c|c|}
\hline Treatment & $50^{\circ} \mathrm{C}, \mathrm{BS}$ & $50^{\circ} \mathrm{C}, \mathrm{DS}$ & $40^{\circ} \mathrm{C}, \mathrm{BS}$ & $40^{\circ} \mathrm{C}, \mathrm{DS}$ \\
\hline \multirow[t]{2}{*}{ Starter cultures } & S. thermophilus & S. thermophilus & S. thermophilus & S. thermophilus \\
\hline & L. helveticus & L. helveticus & L. he/veticus & L. helveticus \\
\hline Max scald & $50^{\circ} \mathrm{C}$ & $50^{\circ} \mathrm{C}$ & $40^{\circ} \mathrm{C}$ & $40^{\circ} \mathrm{C}$ \\
\hline Curd handling & Pre-press and mold & Cheddaring of curd & Pre-press and mold & Cheddaring of curd \\
\hline Salting method & Brine & Dry salt at $1.45 \%(\mathrm{w} / \mathrm{w})$ & Brine & Dry salt at $1.45 \%(\mathrm{w} / \mathrm{w})$ \\
\hline
\end{tabular}

(Table 1). Curds were cooked at a rate of $0.5^{\circ} \mathrm{C} / \mathrm{min}$ from 34 to $45^{\circ} \mathrm{C}$ and at $1^{\circ} \mathrm{C} / \mathrm{min}$ from $45^{\circ} \mathrm{C}$ to maximum scald $\left(50^{\circ} \mathrm{C}\right)$ where appropriate. Curds were pitched at $\mathrm{pH}$ 6.3.

Brine salted (BS) cheeses were pre-pressed under whey at $5.4 \mathrm{kPa}$ for $10 \mathrm{~min}$ after which the curd was placed in $10 \mathrm{~kg}$ round molds and pressed under increasing pressure up to $35.2 \mathrm{kPa}$. At $\mathrm{pH} 5.3$, the cheeses were de-molded and placed for $21 \mathrm{~h}$ in a saturated brine solution $[23 \%(\mathrm{w} / \mathrm{w}) \mathrm{NaCl}, 0.2 \%(\mathrm{w} / \mathrm{w}) \mathrm{Ca}, \mathrm{pH}$ $\left.5.2,10^{\circ} \mathrm{C}\right]$.

Dry salted (DS) cheeses were drained and the curd cheddared, milled at $\mathrm{pH} 5.3$, salted at a rate of $1.45 \%(\mathrm{w} / \mathrm{w})$ and pressed overnight on a horizontal press at $264.6 \mathrm{kPa}$ in $20 \mathrm{~kg}$ blocks. All cheeses were vacuum packed post brine/pressing and ripened at $8^{\circ} \mathrm{C}$ for up to 230 days.

\section{Macroscopic pH Measurement}

A standard procedure to measure the $\mathrm{pH}$ of cheese (BS 7705:1976) was used. Briefly, a slurry was created from a cheese sample using distilled water at $20^{\circ} \mathrm{C}$ and the $\mathrm{pH}$ of the cheese suspension was recorded with a standard laboratory electrode-based $\mathrm{pH}$ meter. All samples were measured in duplicate and the mean values are provided.

\section{Fluorescence Microscopy Sample Preparation}

C-SNARF-4 calibration samples were prepared by mixing $10 \mu \mathrm{l}$ of $1 \mathrm{mM}$ C-SNARF-4 in DMSO stock solution with $90 \mu \mathrm{l}$ of $100 \mathrm{mM}$ sodium citrate buffer adjusted to $\mathrm{pH} 3.0-7.0$. A $20 \mu \mathrm{l}$ drop of the solution was placed on a glass coverslip and imaged with an inverted confocal microscope.

C-SNARF-4 stained cheese samples were prepared by cutting a small piece (about $5 \times 5 \times 0.5 \mathrm{~mm}$ ) from the bulk of the cheese, the piece was then placed on a glass coverslip with a $20 \mu \mathrm{l}$ drop of fluorophore solution ( $10 \mu \mathrm{l}$ of $1 \mathrm{mM} \mathrm{C-SNARF-4} \mathrm{in} \mathrm{DMSO}$ stock solution mixed with $90 \mu \mathrm{l}$ of deionized water). Additionally, cheese samples without the fluorescent probe were prepared to assess the amount of cheese autofluorescence.

Oregon Green 488 (Molecular Probes, D-7172) stained calibration samples were prepared by immersing a small piece of cheese in a $40 \mu \mathrm{l}$ drop of Oregon Green 488 buffered solution $(20 \mu \mathrm{l}$ of $50 \mu \mathrm{M}$ dye in deionized water, mixed with $20 \mu \mathrm{l}$ of $100 \mathrm{mM}$ sodium citrate buffer adjusted to $\mathrm{pH} 3.0-6.0$ ) on a glass coverslip and imaged with an inverted microscope.

For cheese $\mathrm{pH}$ measurements the same protocol was followed, but the citrate buffer was replaced by an equal amount of deionized water to maintain the same dye concentration as in the calibration samples. To assess the cheese autofluorescence, cheese samples without the fluorescent probe were also prepared.

\section{Fluorescence Image Acquisition}

Spectrally resolved fluorescence measurements and excitationemission spectra measurements of C-SNARF-4 were performed with Leica TCS SP8 X confocal laser scanning microscope based on Leica DMI6000 inverted fluorescent microscope (Leica Microsystems CMS GmbH) equipped with White Light Laser (freely tunable excitation in the spectral range 470$670 \mathrm{~nm}$ ), acousto-optical beam splitter and hybrid detectors with a LightGate option. The Acousto-Optical Beam Modulator (AOBM) was set in the mode of constant output power over the whole working interval that allows the constant intensity of the excitation laser beam for all excitation wavelengths impinging on the sample. The emission spectra were collected sequentially with internal spectral detector, with spectral bandwidth of $20 \mathrm{~nm}$ and step size $20 \mathrm{~nm}$ in the range $500-720 \mathrm{~nm}$ with the starting offset $10 \mathrm{~nm}$ from the excitation wavelength. We did not take into account cross-excitation and possible fluorescence emitted in the range less than $10 \mathrm{~nm}$ from the excitation wavelength. The image acquisition and analysis were performed in software LAS AF 3.1.2 (Leica Microsystems $\mathrm{GmbH}$ ). Data were averaged from $200 \times 200 \mu \mathrm{m}$ area, approximately $30 \mu \mathrm{m}$ deep in the calibration solution with a $20 \times / 0.75$ immersion objective lens.

\section{Fluorescence Lifetime Measurements}

Lifetime measurements of C-SNARF-4 calibration samples and C-SNARF-4 stained cheese samples were performed with Olympus FV1200 confocal laser scanning microscope based on Olympus IX83 inverted fluorescence microscope (Olympus Europe $\mathrm{GmbH}$ ) equipped with two single photon counting hybrid detectors, two channel time correlator and $485 \mathrm{~nm}$ pulsed picosecond laser (PicoQuant $\mathrm{GmbH}$ ). The laser repetition rate was set to 40 $\mathrm{MHz}$ and the laser power was adjusted to keep the mean detected photon rate below $2 \%$ of the laser repetition rate. Two spectral ranges were measured simultaneously (channel 1 corresponding to $570-620 \mathrm{~nm}$, channel 2 corresponding to $650-700 \mathrm{~nm}$ ). Typically, calibration data were averaged from $100 \times 100 \mu \mathrm{m}$ area, approximately $30 \mu \mathrm{m}$ deep in the calibration solution with a $60 \times / 1.2$ water immersion objective lens. Typically, $120 \mathrm{~s}$ acquisition was necessary to accumulate $10^{5}$ detection events. When measuring cheese samples the focus was set to the cheese surface, i.e., the plane of the strongest fluorescence. The measured fluorescence decay curves were fitted to a two- or three-component 
exponential model (a sum of two or three exponential decays) according to

$$
I(t)=\sum_{n=1}^{N} a_{n} e^{-t / \tau_{n}}+b
$$

using the PicoQuant SymPhoTime64 TCSPC fitting software. Here $I(t)$ is the model fluorescence intensity, $N$ is the number of components of the model, $I_{n}$ and $\tau_{n}$ are the intensity and the lifetime of the $n$-th component of the model respectively ( $n=1,2,3$; lifetimes $\tau_{n}$ arranged in ascending order) and $b$ is the mean background. The fluorescence images were acquired at $512 \times 512$ pixels that were binned to $256 \times 256$ pixels for lifetime calculations.

Lifetime measurements of Oregon Green 488 stained cheese samples were performed with Zeiss LSM780 NLO confocal microscope based on Axio Observer. Z1 inverted microscope (Carl Zeiss MicroImaging $\mathrm{GmbH}$ ) equipped with Chameleon Vision femtosecond pulsed IR laser (Coherent Inc.) tuned to $860 \mathrm{~nm}$ and Becker and Hickl hybrid detector (HPM-100-40) and TCSPC hardware and software (SPC-150, Becker \& Hickl $\mathrm{GmbH})$. The repetition rate of the pulsed laser was fixed to 80 $\mathrm{MHz}$. Fluorescence was collected through an IR blocking filter and 580-640 nm emission filter with a Plan Apo $63 \times / 1.4$ oil IR objective lens. Fluorescence lifetimes were calculated from $512 \times$ 512 pixel images binned to $256 \times 256$ pixels. The measured fluorescence decay curves were analyzed in terms of two-exponential model according to Equation (1) and the mean lifetime was then calculated according to

TABLE 2 | Macroscopic pH values of cheese trials at day 223 of ripening.

\begin{tabular}{lcccc}
\hline Sample & vat 1 & vat 2 & vat 3 & vat 4 \\
\hline Treatment & $50^{\circ} \mathrm{C}, \mathrm{BS}$ & $50^{\circ} \mathrm{C}, \mathrm{DS}$ & $40^{\circ} \mathrm{C}, \mathrm{BS}$ & $40^{\circ} \mathrm{C}, \mathrm{DS}$ \\
$\mathrm{pH}$ & $5.20 \pm 0.01$ & $5.31 \pm 0.01$ & $4.99 \pm 0.01$ & $5.09 \pm 0.01$
\end{tabular}

$$
\tau_{m}=\frac{\sum_{n=1}^{N} a_{n} \tau_{n}}{\sum_{n=1}^{N} a_{n}}
$$

using the Beker \& Hickl SPCImage software. Here $\tau_{m}$ is the mean lifetime, other symbols are defined in Equation (1).

\section{Results}

\section{Macroscopic pH Of Natural Cheese Samples}

The results obtained through the standard measurement with a $\mathrm{pH}$ electrode of homogenized slurry samples are summarized in Table 2. It is evident that the cheese pHs vary depending on the cheese manufacture treatments but all are within the range 4.99-5.31 and are typical of the $\mathrm{pH}$ of many natural cheese types However, what this method does not measure is the degree and pattern of variability in $\mathrm{pH}$ within each cheese matrix. It is possible to get approximate measurement by direct measurement with a $\mathrm{pH}$ electrode of points within the cheese matrix but this is not sufficiently accurate to create a full $\mathrm{pH}$ map of the matrix and to relate the $\mathrm{pH}$ map to localized compositional, biochemical and microbial differences within the cheese matrix.

\section{C-SNARF-4 Emission Spectra}

The $\mathrm{pH}$ dependence of the probe's emission spectrum is summarized in Figure 2 for two excitation wavelengths ( $480 \mathrm{~nm}$ - the wavelength used for subsequent lifetime measurements; and $530 \mathrm{~nm}$ - the wavelength of most efficient excitation) in the $\mathrm{pH}$ range 3.0-7.0. The $\mathrm{pH}$ dependence of the ratio of the intensities of the two emission peaks (centered at 590 and $660 \mathrm{~nm}$, respectively) is shown in Figure 3.

\section{C-SNARF-4 Excitation-Emission Spectra}

The excitation-emission scans (Figure 4) indicate almost no $\mathrm{pH}$ sensitivity of the dye in the $\mathrm{pH}$ range of 3.7-5.0 (the Figures 4A-F show the same spectral footprint regardless of $\mathrm{pH}$ ). At $\mathrm{pH} 5$ and above (Figures $\mathbf{4 F}-\mathbf{J}$ ) there is an apparent trend
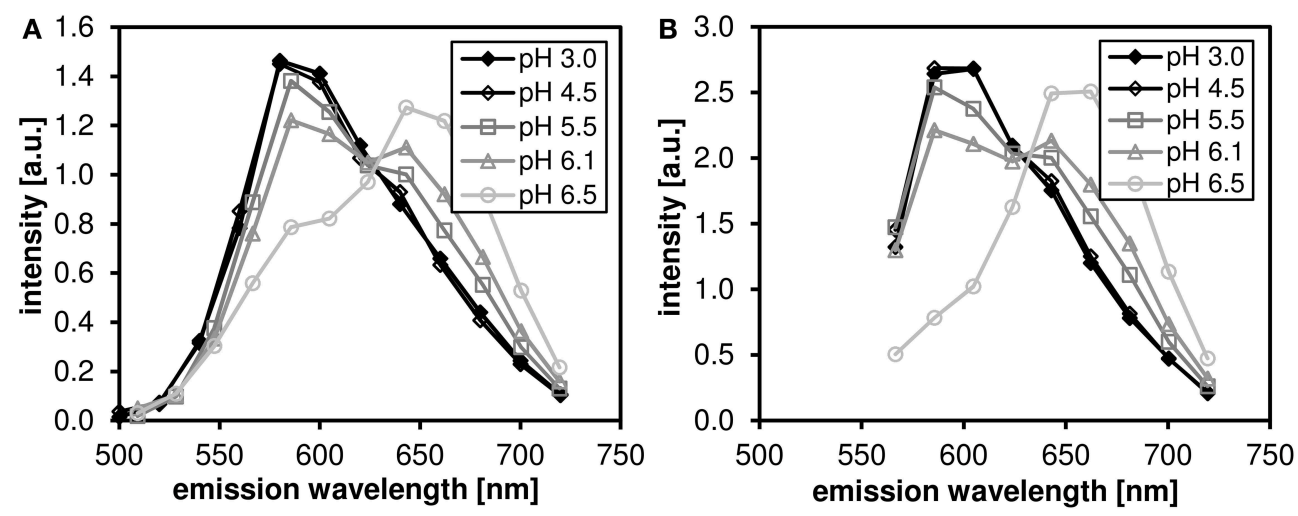

FIGURE 2 | The pH-dependent emission spectra of C-SNARF-4 in water. Measured in the pH range $3.0-6.5$ at two excitation wavelengths: (A) 488 nm, (B) $530 \mathrm{~nm}$. Note that the spectra do not change significantly below pH 4.5 . 
in the spectra that manifests the $\mathrm{pH}$ sensitivity of the probe. However, introducing time gating (discarding photons that arrive within $1 \mathrm{~ns}$ of the excitation pulse) modifies the spectral footprint of the (Figure 5) suggesting that lifetime imaging (FLIM) may enhance $\mathrm{pH}$ sensitivity at lower $\mathrm{pH}$.

\section{C-SNARF-4 Fluorescence Lifetimes In Aqueous Solution}

We performed calibration measurements of C-SNARF-4 dye in aqueous $\mathrm{pH}$ buffers in the range of $\mathrm{pH} 3.0-7.0$ and measured the fluorescence lifetimes in two spectral bands: channel 1 corresponds to $570-620 \mathrm{~nm}$, and channel 2 corresponds to

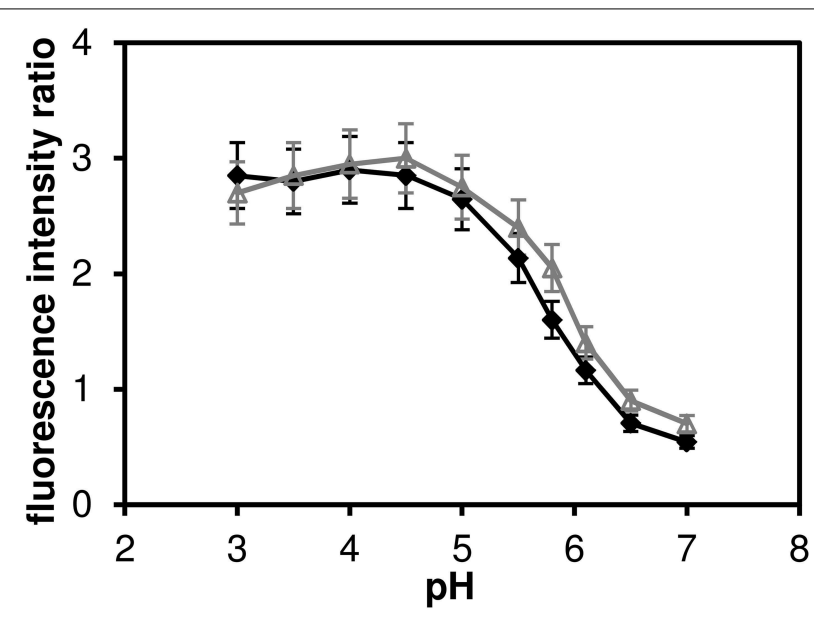

FIGURE 3 | The ratio of fluorescence intensities $I_{590} I_{660}$ of C-SNARF-4 ratiometric $\mathbf{~} \mathrm{H}$ probe in water. Measured in the $\mathrm{pH}$ range of 3.0-7.0, excited at $480 \mathrm{~nm}$ (solid symbols) ad at $530 \mathrm{~nm}$ (open symbols). The widths of the two emission bands (centered at 590 and $660 \mathrm{~nm}$, respectively) are $60 \mathrm{~nm}$.
650-700 nm. These two spectral bands were chosen according to the two emission peaks of the dye used for ratiometric $\mathrm{pH}$ imaging.

While a two-exponential model fits sufficiently well the decay curves of channel 2 (far red fluorescence), three-exponential model is necessary for analyzing channel 1 (orange fluorescence). The results of the multi-exponential fitting procedure, i.e., $\mathrm{pH}$ dependence of the individual lifetime components are shown in Figure 6A (channel 1) and Figure 6B (channel 2). While the microscopic origin of the individual components is unknown, they can be used empirically to measure local $\mathrm{pH}$, as long as they display sufficient sensitivity (the lifetime changes monotonically with $\mathrm{pH}$ and this change is bigger than the statistical error of the measurement). None of the channel 1 lifetime components displayed pronounced sensitivity toward $\mathrm{pH}$. On the other hand, the long lifetime component of the channel 2 decay curves shows
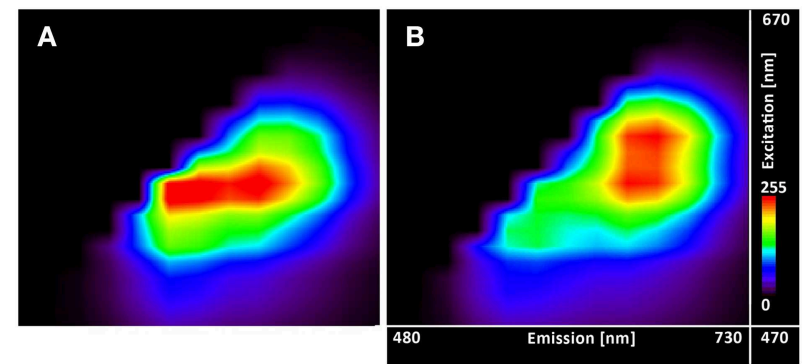

FIGURE 5 | Excitation-emission lambda scans of the C-SNARF-4 in water. Measured at $\mathrm{pH} 5.5$ (A) without timegate and (B) with timegate 1 ns, i.e., all photons arriving within the first nanosecond after the excitation pulse are discarded. Vertical axis shows excitation wavelengths of White Light Laser (470-670 nm), horizontal axis displays emission spectra $(480-730 \mathrm{~nm})$
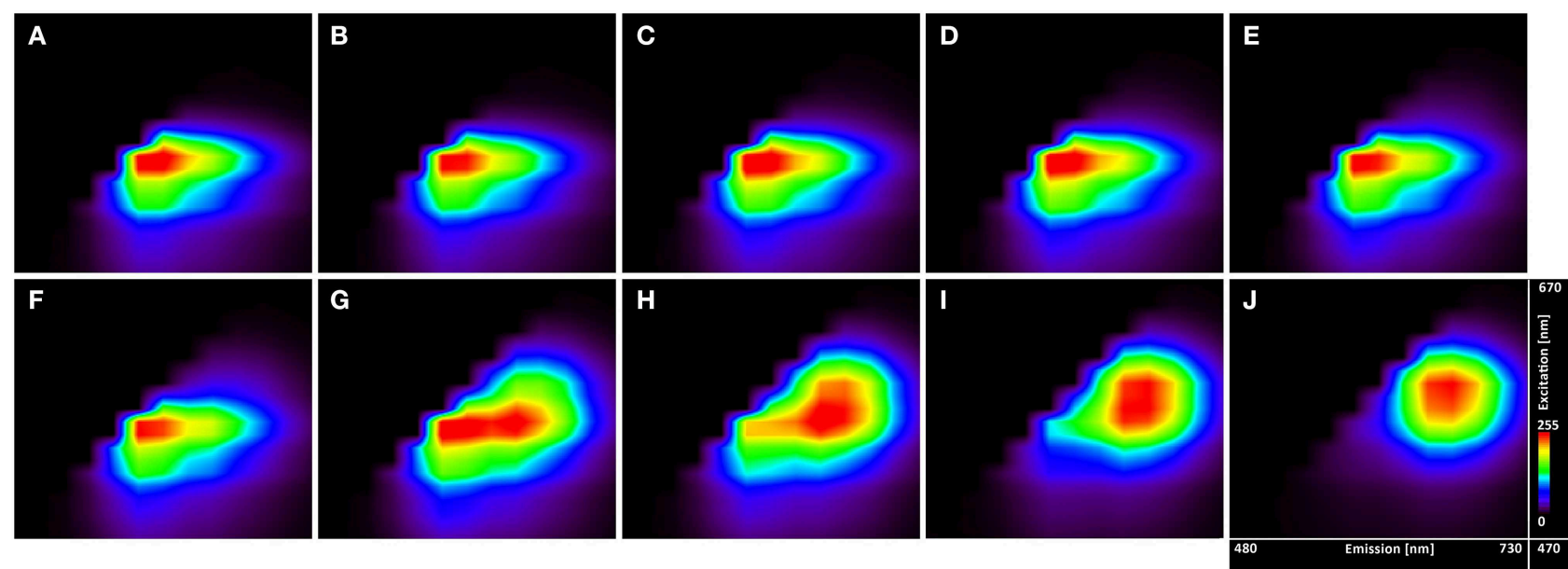

FIGURE 4 | Excitation-emission lambda scans of the C-SNARF-4 in water buffered to different $\mathbf{p H}$. Vertical axis shows excitation wavelengths of White Light Laser (470-670 nm), whereas horizontal axis displays emission spectra (480-730 nm) of C-SNARF-4 in different pH: (A) 3.7, (B) 4.0, (C) 4.3,
(D) 4.5, (E) 4.8, (F) 5.0, (G) 5.5, (H) 5.8, (I) 6.1, and (J) 6.5. Increase of pH value results in moving maximum emission from the blue part of spectra toward the red part of spectra with forming two peaks horizontally for $\mathrm{pH} 5.5$ and quasi-symmetrical emission for $\mathrm{pH} 6.1$ and 6.5. 


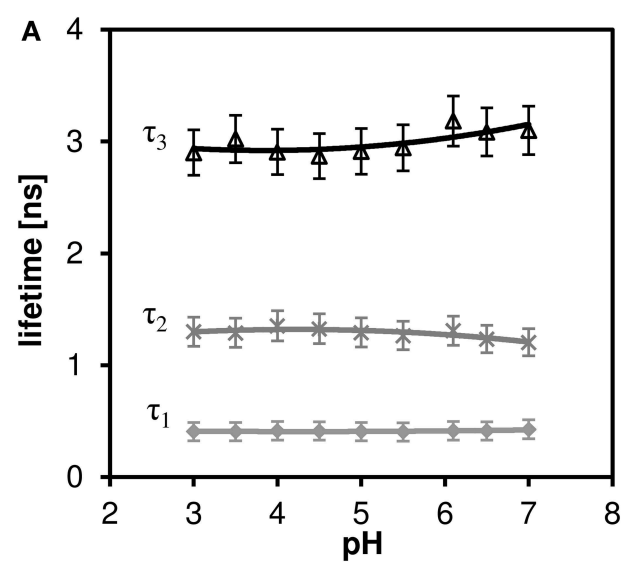

FIGURE 6 | The effect of $\mathrm{pH}$ on the fluorescence lifetime of C-SNARF-4 in water. (A) Channel 1 (570-620 nm emission), three-component model, none of the lifetime components shows

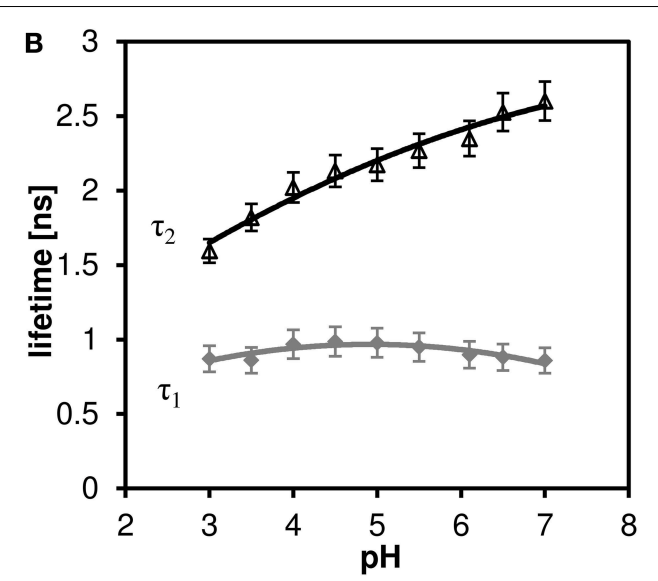

pronounced dependence. (B) Channel 2 (650-700 nm emission), two-component model, the longer lifetime component $\tau_{2}$ shows pronounced $\mathrm{pH}$ dependence.
TABLE 3 | The fluorescence lifetimes of cheese matrix stained with C-SNARF-4 fluorescent dye, channel 2, three-component exponential model.

\begin{tabular}{cccc}
\hline & $\boldsymbol{\tau}_{\mathbf{1}}$ [ns] & $\boldsymbol{\tau}_{\mathbf{2}}$ [ns] & $\boldsymbol{\tau}_{\mathbf{3}}$ [ns] \\
\hline vat 1 & $0.62 \pm 0.05$ & $1.65 \pm 0.09$ & $3.71 \pm 0.18$ \\
vat 2 & $0.61 \pm 0.02$ & $1.63 \pm 0.03$ & $3.69 \pm 0.16$ \\
vat 3 & $0.56 \pm 0.01$ & $1.59 \pm 0.02$ & $3.65 \pm 0.08$ \\
vat 4 & $0.59 \pm 0.03$ & $1.60 \pm 0.04$ & $3.62 \pm 0.09$ \\
\hline
\end{tabular}

Average lifetime \pm standard deviation calculated from 6 to 8 measurements.

pronounced $\mathrm{pH}$ dependence in the whole measured $\mathrm{pH}$ range (the upper curve in Figure 6B). Mean fluorescence lifetime $\tau_{m}$ calculated according to Equation (2) does not show pronounced $\mathrm{pH}$ dependence, thus two-exponential fitting is necessary to extract the $\mathrm{pH}$ information.

\section{Microscopic pH Of Cheese Samples Measured With C-SNARF-4}

Based on calibration data we tried to utilize the $\mathrm{pH}$ dependence of C-SNARF-4 fluorescence in the red spectral channel for cheese $\mathrm{pH}$ measurements on the microscopic scale. However, both the fluorescence intensity and the fluorescence lifetime distribution were essentially homogeneous across the sample. We have also observed a strong affinity of the dye toward the cheese surface.

We measured a total of 4 cheeses (designated vat 1 to vat 4), each multiple times. Surprisingly, a new decay component with relatively long lifetime $\left(\tau_{3} \geq 3.5 \mathrm{~ns}\right)$ appeared (Table 3). Moreover, the $\tau_{2}$ component, which is assumed to correspond to the $\mathrm{pH}$ dependent component of the calibration data, has significantly shorter lifetime than the value deduced from calibration (expected value is about $2.2 \mathrm{~ns}$ for $\mathrm{pH} 4.5$ ). Also the fastest component ( $\tau_{1} \approx 0.7 \mathrm{~ns}$ ) is significantly shorter, than the corresponding lifetime from calibration (about $0.9 \mathrm{~ns}$ ). Thus, in our scenario the C-SNARF-4 probe did not prove itself as a useful probe of the local $\mathrm{pH}$.

Control experiments performed on cheese samples without fluorescent probe revealed, that the cheese autofluorescence is almost two orders of magnitude weaker than C-SNARF-4 fluorescence, and its lifetime is significantly longer (over $6 \mathrm{~ns}$ ) than that of the fluorescent probe, so that the autofluorescence does not interfere with our measurements.

\section{Oregon Green 488 Fluorescence Lifetimes And Microscopic pH Measurements}

Oregon Green 488 displays single emission peak whose position is independent of local environment. Thus fluorescence lifetime (FLIM) must be employed in order to measure the local $\mathrm{pH}$ variation. The mean fluorescence lifetime of the Oregon Green 488 excited by $860 \mathrm{~nm}$ pulsed laser at $80 \mathrm{MHz}$ and detected in the red emission band was determined to be $3.9 \pm 0.1 \mathrm{~ns}$ in aqueous buffer at $\mathrm{pH} 7$. When the dye is applied to the cheese matrix, the lifetime shortens significantly, but the $\mathrm{pH}$ sensitivity of the dye is preserved. Figure 7A shows representative FLIM images of cheese matrix buffered to $\mathrm{pH}$ 3.0-6.0 and stained with Oregon Green 488 . The $\mathrm{pH}$ sensitivity of the dye is immediately apparent from the change of the pseudocolor encoding local fluorescence lifetime. The mean lifetime $\tau_{m}$ calculated as a mean value from several fields of view is plotted against the buffer $\mathrm{pH}$ in Figure $7 \mathbf{B}$ (solid symbols and a linear least-squares fit). The mean lifetime of the dye changes by more than $1 \mathrm{~ns}$ in the studied $\mathrm{pH}$ range.

To measure the local $\mathrm{pH}$ of cheese the dye is applied to the cheese matrix without a $\mathrm{pH}$ buffer and the same procedure is followed. From the acquired FLIM image and the linear fit of the calibration series the average $\mathrm{pH}$ of vat 2 cheese sample after 223 days of ripening was determined to $5.2 \pm 0.2$ (Figure 7B, open symbol). The local variations of the fluorescence lifetime (and thus the $\mathrm{pH}$ ) in a natural cheese sample are shown in greater detail in Figure 8. We note that application of the dye to the non-buffered cheese produced no measurable macroscopic 

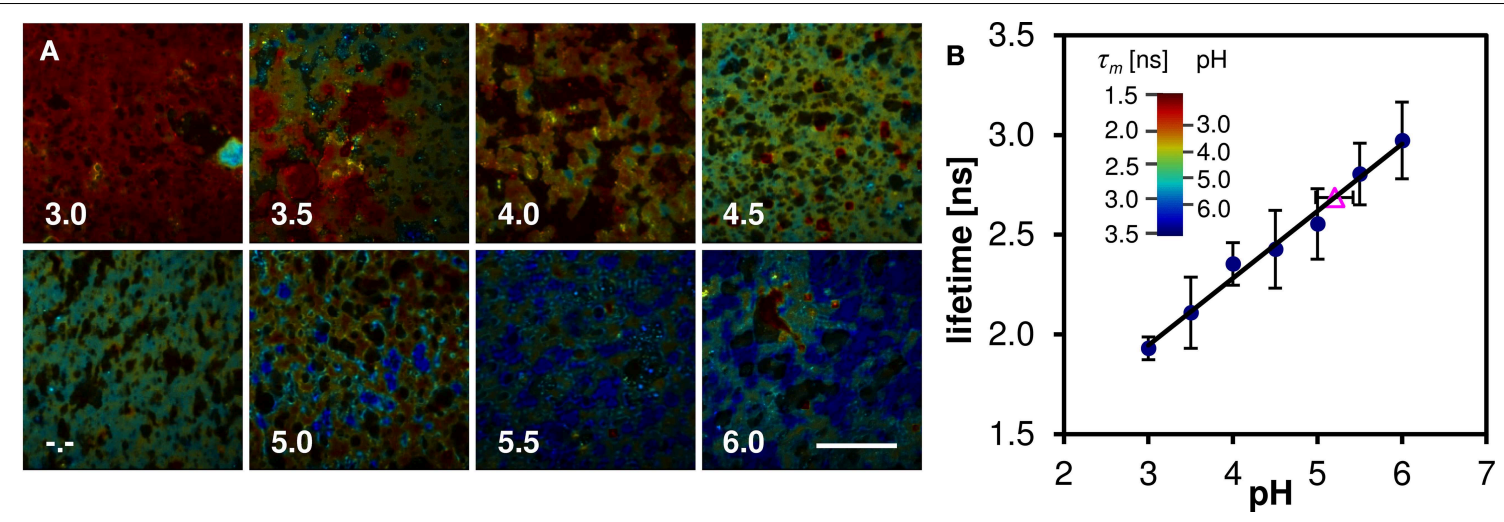

FIGURE 7 | Lifetime measurements of cheese stained with Oregon Green 488 buffered to $\mathbf{p H}$ 3.0-6.0. (A) FLIM images, the pseudocolor represents the mean lifetime $\tau_{m}$, intensity represents photon count, captions denote the $\mathrm{pH}$ of the buffer solution (-.denotes unbuffered sample). Scalebar $50 \mu \mathrm{m}$. (B) The $\mathrm{pH}$ dependence

of mean lifetime $\tau_{m}$ calculated from the FLIM images (solid symbols), and the mean lifetime of stained cheese without $\mathrm{pH}$ buffer (open triangle). Error bars represent the standard deviation, mainly caused by sample inhomogeneity. The inset shows the pseudocolor scale of the FLIM images calibrated both in lifetime and $\mathrm{pH}$ values.

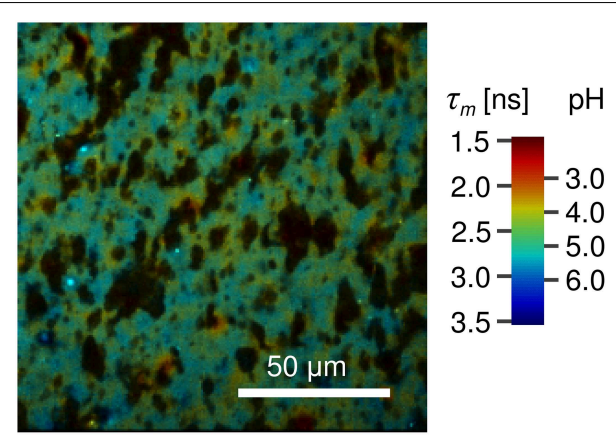

FIGURE 8 | FLIM image of natural cheese sample stained with Oregon Green 488. Shown are apparent local variations of fluorescence lifetime and thus the $\mathrm{pH}$. Localized spots with $\mathrm{pH}$ as low as 4.0 are observed. The dark areas most likely represent fat within the cheese matrix. The pseudocolor scale of the FLIM images is calibrated both in lifetime and $\mathrm{pH}$ values.

$\mathrm{pH}$ change, as measured by a macroscopic $\mathrm{pH}$ electrode method.

\section{Discussion}

We have confirmed that the C-SNARF-4 fluorescent $\mathrm{pH}$ probe may be used as a reliable ratiometric $\mathrm{pH}$ indicator at $\mathrm{pH} 5.5$ and above (Figure 3). Essentially, the emission spectra do not depend on the excitation wavelength in the range $480-530 \mathrm{~nm}$, as illustrated in Figure 2. However, the range of interest in cheese research is usually at lower $\mathrm{pH}$ values, generally around 4.8-6.0 (with some exceptions). In this range the traditional ratiometric approach does not provide sufficient sensitivity.

Excitation-emissions scans (Figure 4) show that the results of ratiometric $\mathrm{pH}$ measurements are largely insensitive to the excitation wavelength, but utilizing the lifetime information may extend the useful range of the dye toward lower pH's (Figure 5).
We have observed and measured the $\mathrm{pH}$ dependence of fluorescence lifetime of C-SNARF-4 dye, which extends through the $\mathrm{pH}$ range that can be sensed toward more acidic values (down to $\mathrm{pH} \approx 3$ ), part of this range which may be encountered in cheese research. From the calibration curve $\tau_{2}$ in Figure 6B) one can clearly see the relationship between $\mathrm{pH}$ and fluorescence lifetime. But the calibrated lifetime- $\mathrm{pH}$ dependence performed in aqueous solutions is not directly applicable to cheese matrix results, as a new long-lifetime component appears $\tau_{3}$ in Table 3 ) and the lifetimes of the other two components are significantly shortened in such a protein-rich environment. More specifically, the $\tau_{2}$ component measured on cheese samples (about $1.7 \mathrm{~ns}$, Table 3) would correspond to $\mathrm{pH}$ below 4.0 (according to the calibration curve $\tau_{2}$ in Figure 6B), which is much lower than the reference macroscopic value (about $\mathrm{pH}$ 5.0, Table 2). A similar interaction of SNARF-based dye with proteins was described previously (Srivastava and Krishnamoorthy, 1997). We also note that we observed strong affinity of the fluorescent probe toward the protein-rich cheese surface, which changes the dye's photophysical properties significantly and alter its $\mathrm{pH}$ response. Both the spectral and lifetime signatures of the stained protein matrix do not correspond to the calibration values at any $\mathrm{pH}$.

Moreover, we observed that both the fluorescence intensity and lifetimes are essentially homogeneous across the sample surface (as shown by the small standard deviations in Table 3). The expected $\mathrm{pH}$ variation between samples is less than 0.5 and cannot be reliably detected by the C-SNARF-4 dye.

Similarly, we also applied the same methodology to other $\mathrm{pH}$ sensitive fluorescent dyes, namely BCECF-AM, FITC, and Acridine Orange, but again we observed little to no lifetime-pH sensitivity when applied to cheese matrix (data not shown).

On the other hand, our results suggest that applying the FLIM microscopic technique to the Oregon Green 488 fluorescent dye resulted in an accurate method of determination of $\mathrm{pH}$ in the cheese samples (the main results are summarized in Figure 7). The average value of $\mathrm{pH} 5.2 \pm 0.2$ is in good agreement with the 
macroscopic pH 5.3 (Table 2). In addition micro-heterogeneity in $\mathrm{pH}$ is apparent in the cheese matrix as show in Figure 8 with $\mathrm{pH}$ ranging between 4.0 and 5.5 depending on location. This is particularly interesting as it shows the $\mathrm{pH}$ of cheese matrix is not homogenous but contains localized variation of $\mathrm{pH}$. This may be due to localized differences in the aqueous phase or concentrations of constituents of the aqueous phase including lactose, lactate, minerals or salt. It may also be influenced by variations in buffering capacity of the surrounding cheese matrix. It also poses questions regarding the influence of microbial colonies on pH micro heterogeneity. Other authors (Lopez et al., 2006, 2007; Pereira et al., 2009) reported bacterial colonies to be located at the protein fat interface, with the bacterial cells always being in contact with the fat globule membrane. Although the study of (Jeanson et al., 2013) showed no differences in $\mathrm{pH}$ around microbial colonies, that study differed in that it was conducted on an unripened non-fat UF model cheese system rather than on a

\section{References}

Agronskaia, A. V., Tertoolen, L., and Gerritsen, H. C. (2004). Fast fluorescence lifetime imaging of calcium in living cells. J. Biomed. Opt. 9, 1230-1237. doi: $10.1117 / 1.1806472$

Becker, W. (2005). The bh TCSPC Handbook. Berlin: Becker \& Hickl GmbH.

Creamer, L. K., Lawrence, R. C., and Gilles, J. (1985). Effect of acidification of cheese milk on the resultant Cheddar cheese. N. Z. J. Dairy Sci. Technol. 20, 185-203.

Dunn, K. W., Maxfield, F. R., Whitaker, J. W., and Haugland, R. P. (1991). Fluorescence excitation ratio $\mathrm{pH}$ measurements of lysosomal ph using laser scanning confocal microscopy. Biophys. J. 59, 345a.

Euston, S. R., Piska, I., Wium, H., and Qvist, K. B. (2002). Controlling the structure and rheological properties of model cheese systems. Aust. J. Dairy Technol. 57, 91-98. Available online at: https:// www.diaa.asn.au/publications/australian-journal-of-dairy-technology/articles/ article/australian-journal-of-dairy-technology/23-adjt-articles/640-controllingthe-structure-and-rheological-properties-of-model-cheese-systems

Feeney, E. P., Guinee, T. P., and Fox, P. F. (2002). Effect of pH and calcium concentration on proteolysis in mozzarella cheese. J. Dairy Sci. 85, 1646-1654. doi: 10.3168/jds.S0022-0302(02)74237-9

Foulquié Moreno, M. R., Rea, M. C., Cogan, T. M., and De Vuyst, L. (2003). Applicability of a bacteriocin-producing Enterococcus faecium as a co-culture in Cheddar cheese manufacture. Int. J. Food Microbiol. 81, 73-84. doi: 10.1016/S0168-1605(02)00167-8

Fox, P. F., McSweeney, P. L. H., Cogan, T. M., and Guinee, T. P. (2000). Fundamentals of Cheese Science. Berlin: Springer Science \& Business.

Gardini, F., Martuscelli, M., Caruso, M. C., Galgano, F., Crudele, M. A., Favati, F., et al. (2001). Effects of $\mathrm{pH}$, temperature and $\mathrm{NaCl}$ concentration on the growth kinetics, proteolytic activity and biogenic amine production of Enterococcus faecalis. Int. J. Food Microbiol. 64, 105-117. doi: 10.1016/S0168-1605(00) 00445-1

Garnot, P., Molle, D., and Piot, M. (1987). Influence of pH, type of enzyme and ultrafiltration on the retention of milk clotting enzymes in Camembert cheese. J. Dairy Res. 54, 315-320.

Grufferty, M. B., and Fox, P. F. (1988). Milk alkaline proteinase. J. Dairy Res. 55, 609-630.

Han, J., and Burgess, K. (2010). Fluorescent indicators for intracellular pH. Chem. Rev. 110, 2709-2728. doi: 10.1021/cr900249z

Haugland, R. P. (2002). Handbook of Fluorescent Probes and Research Chemicals, 9th Edn. Eugene: Molecular Probes, Inc.

Holmes, D. G., Duersch, J. W., and Ernstrom, C. A. (1977). Distribution of milk clotting enzymes between curd and whey and their survival during ripened natural cheese matrix, and the microbial colonies were lactococci rather than thermophillic species.

The current study has focused on applying the developed method to determining the micro heterogeneity within one of the cheese types (vat 2). It is envisaged that future work would focus on using the developed method to determine whether manufacture processes influence $\mathrm{pH}$ at local level within different cheese matrices and whether different cheese types may have different patterns of micro heterogeneity. We also expect that this methodology can be employed to examine also wide range of different types of food products with sufficient moisture content.

\section{Acknowledgments}

We acknowledge funding from the Dairy Levy Trust and the Czech Science Foundation (grant no. 14-15272P).

cheddar cheese making. J. Dairy Sci. 60, 862-869. doi: 10.3168/jds.S00220302(77)83955-6

Hunter, R. C., and Beveridge, T. J. (2005). Application of a pH-sensitive fluoroprobe (C-SNARF-4) for $\mathrm{pH}$ microenvironment analysis in Pseudomonas aeruginosa. Biofilms 71, 2501-2510. doi: 10.1128/AEM.71. 5.2501

Inoue, S. (1995). "Foundations of confocal scanned imaging in light microscopy," in Handbook of Biological Confocal Microscopy, 2nd Edn., ed J. B. Pawley (New york, NY: Plenum Press), 1.

Jeanson, S., Chadœuf, J., Madec, M. N., Aly, S., Floury, J., Brocklehurst, T. F., et al. (2011). Spatial distribution of bacterial colonies in a model cheese. Appl. Environ. Microbiol. 77, 1493-1500. doi: 10.1128/AEM.02233-10

Jeanson, S., Floury, J., Issulahi, A., Madec, M. N., Thierry, A., and Lortal, S. (2013). Microgradients of $\mathrm{pH}$ do not occur around Lactococcus colonies in a model cheese. Appl. Environ. Microbiol. 79, 6516-6518. doi: 10.1128/AEM. 01678-13

Kajfasz, J. K., and Quivey, R. G. Jr. (2011). "Responses of lactic acid bacteria to acid stress," in Stress Responses of Lactic Acid Bacteria SE - 2 Food Microbiology and Food Safety, eds E. Tsakalidou and K. Papadimitriou (New York, NY: Springer US), 23-53.

Kilcast, D., and Angus, F. (eds.). (2007). Reducing Salt in Foods: Practical Strategies, $1 s t$ Edn. Cambridge, UK: Woodhead Publishing Ltd.

Lakowicz, J. R. (2006). Principles of Fluorescence Spectroscopy, 3rd Edn. Berlin: Springer Science and Bussiness.

Laloy, E., Vuillemard, J. C., El Soda, M. E., and Simard, R. E. (1996). Influence of the fat content of Cheddar cheese on retention and localization of starters. Int. Dairy J. 6, 729-740. doi: 10.1016/0958-6946(95)00068-2

Lopez, C., Camier, B., and Gassi, J.-Y. (2007). Development of the milk fat microstructure during the manufacture and ripening of Emmental cheese observed by confocal laser scanning microscopy. Int. Dairy J. 17, 235-247. doi: 10.1016/j.idairyj.2005.12.015

Lopez, C., Maillard, M. B., Briard-Bion, V., Camier, B., and Hannon, J. A. (2006). Lipolysis during ripening of emmental cheese considering organization of fat and preferential localization of bacteria. J. Agric. Food Chem. 54, 5855-5867. doi: $10.1021 /$ jf060214l

McSweeney, P. L. H. (2004a). Biochemistry of cheese ripening. Int. J. Dairy Technol. 57, 127-144. doi: 10.1111/j.1471-0307.2004.00147.x

McSweeney, P. L. H. (2004b). "Biochemistry of cheese ripening: introduction and overview," in Cheese: Chemistry, Physics and Microbiology Cheese: Chemistry, Physics and Microbiology, eds P. F. Fox, P. L. H. McSweeney, T. M. Cogan, and T. P. Guinee (Amsterdam: Elsevier), 347-360.

Meldrum, R. J., Brocklehurst, T. F., Wilson, D. R., and Wilson, P. D. G. (2003). The effects of cell immobilization, $\mathrm{pH}$ and sucrose on the growth 
of Listeria monocytogenes Scott A at $10^{\circ}$ C. Food Microbiol. 20, 97-103. doi: 10.1016/S0740-0020(02)00083-7

Pereira, C. I., Gomes, A. M. P., and Xavier Malcata, F. (2009). Microstructure of cheese: processing, technological and microbiological considerations. Trends Food Sci. Technol. 20, 213-219. doi: 10.1016/j.tifs.2009.02.006

Scott, R. (1981). Cheesemaking Practice. London; New York: Elsevier Applied Science Publishers.

Srivastava, A., and Krishnamoorthy, G. (1997). Time-resolved fluorescence microscopy could correct for probe binding while estimating intracellular $\mathrm{pH}$. Anal. Biochem. 249, 140-146. doi: 10.1006/abio.1997.2164

Stadhouders, J., Hup, G., and van der Waals, C. B. (1977). Determination of calfrennet in cheese. Neth. Milk Dairy J. 31, 3-15.

Sun, Y., Day, R. N., and Periasamy, A. (2011). Investigating protein-protein interactions in living cells using fluorescence lifetime imaging microscopy. Nat. Protoc. 6, 1324-1340. doi: 10.1038/nprot.2011.364

Szmacinski, H., and Lakowicz, J. R. (1993). Optical measurements of pH using fluorescence lifetimes and phase-modulation fluorometry. Anal. Chem. 65, 1668-1674.

Visser, E. M. W. (1977). Contribution of enzymes from rennet starter bacteria and milk to proteolysis and flavor development in Gouda cheese. 1. Description of cheese and aseptic cheesemaking technique. Neth. Milk Dairy J. 30, 120-133.
Whitaker, J. E., Haugland, R. P., Ryan, D., Dunn, K., and Maxfield, F. R. (1991). Dual excitation ph sensitive conjugates of dextran and transferrin for $\mathrm{ph}$ measurement during endocytosis utilizing $514 \mathrm{~nm}$ to $488 \mathrm{~nm}$ excitation ratios. Biophys. J. 59, 358a.

Wimpenny, J. W. T., Leistner, L., Thomas, L. V., Mitchell, A. J., Katsaras, K., and Peetz, P. (1995). Submerged bacterial colonies within food and model systems: their growth, distribution and interactions. Int. J. Food Microbiol. 28, 299-315. doi: 10.1016/0168-1605(95)00065-8

Conflict of Interest Statement: The authors declare that the research was conducted in the absence of any commercial or financial relationships that could be construed as a potential conflict of interest.

Copyright (๑) 2015 Burdikova, Svindrych, Pala, Hickey, Wilkinson, Panek, Auty, Periasamy and Sheehan. This is an open-access article distributed under the terms of the Creative Commons Attribution License (CC BY). The use, distribution or reproduction in other forums is permitted, provided the original author(s) or licensor are credited and that the original publication in this journal is cited, in accordance with accepted academic practice. No use, distribution or reproduction is permitted which does not comply with these terms. 


\section{Diffusion of solutes inside bacterial colonies immobilized in model cheese depends on their physicochemical properties: a time-lapse microscopy study}

OPEN ACCESS

Edited by:

Jean-christophe Augustin,

Ecole Nationale

Vétérinaire d'Alfort, France

Reviewed by:

Antonio Valero,

University of Cordoba, Spain

Louis Coroller,

Université de Brest, France

${ }^{*}$ Correspondence:

Juliane Floury,

UMR Science and Technology of Milk and Eggs, INRA/Agrocampus Ouest,

65 rue de Saint Brieuc,

35042 Rennes Cx, France juliane.floury@agrocampus-ouest.fr

Specialty section:

This article was submitted to

Food Microbiology,

a section of the journal

Frontiers in Microbiology

Received: 05 February 2015

Paper pending published: 18 March 2015

Accepted: 10 April 2015

Published: 30 April 2015

Citation:

Floury J, El Mourdi I, Silva JVC, Lortal S, Thierry A and Jeanson S (2015) Diffusion of solutes inside bacterial colonies immobilized in model cheese depends on their physicochemical properties: a time-lapse microscopy

study. Front. Microbiol. 6:366.

doi: 10.3389/fmicb.2015.00366
Juliane Floury ${ }^{1,2 *}$, Ilham El Mourdi ${ }^{1,2}$, Juliana V. C. Silva ${ }^{1,2}$, Sylvie Lortal ${ }^{1,2}$, Anne Thierry ${ }^{1,2}$ and Sophie Jeanson ${ }^{1,2}$

${ }^{1}$ INRA, UMR1253 Science and Technology of Milk and Eggs, Rennes, France, ${ }^{2}$ Agrocampus Ouest, UMR1253 Science and Technology of Milk and Eggs, Rennes, France

During cheese processing and ripening, bacteria develop as colonies. Substrates and metabolites must then diffuse either from or into the colonies. Exploring how the inner cells of the colony access the substrates or get rid of the products leads to study the diffusion of solutes inside bacterial colonies immobilized in cheese. Diffusion limitations of substrates within the bacterial colony could lead to starvation for the cells in the center of the colony. This study aimed at better understands ripening at the colony level, by investigating how diffusion phenomena inside colonies vary depending on both the physicochemical properties of the solutes and Lactococcus lactis strain. Dextrans $(4,70$, and $155 \mathrm{kDa}$ ) and milk proteins (BSA, lactoferrin and $\alpha_{\mathrm{S} 1}$-casein) of different sizes and physicochemical properties were chosen as model of diffusing solutes, and two L. lactis strains presenting different surface properties were immobilized as colonies in a model cheese. Diffusion of solutes inside and around colonies was experimentally followed by time-lapse confocal microscopy. Dextran solutes diffused inside both lactococci colonies with a non-significantly different effective diffusion coefficient, which depended mainly on size of the solute. However, whereas flexible and neutral hydrophilic polymers such as dextran can diffuse inside colonies whatever its size, none of the three proteins investigated in this study could penetrate inside lactococci colonies. Therefore, the diffusion behavior of macromolecules through bacterial colonies immobilized in a model cheese did not only depends on the size of the diffusing solutes, but also and mainly on their physicochemical properties. Milk caseins are probably first hydrolyzed by the cell wall proteases of $L$. lactis and/or other proteases present in the cheese, and then the generated peptides diffuse inside colonies to be further metabolized into smaller peptides and amino acids by all the cells located inside the colonies.

Keywords: bacterial colony, Lactoccocus lactis, cheese, confocal microscopy, diffusion, milk protein 


\section{Introduction}

During cheese making, regardless of the cheese type, bacteria are immobilized in the curd during the coagulation step, and then grow as colonies spread within the cheese curd. Jeanson et al. (2011) showed that the distribution of Lactococcus lactis colonies was random in a non-fat model cheese. Lactococci are the most used starters in the cheese industry. They are a major actor of ripening which gives the cheese its final sensorial properties. During ripening, they are responsible for the proteolysis, the milk protein breakdown, leading to peptides and amino acids. It is then obvious that the access to nitrogen sources, i.e., proteins and derivates, is of major importance for the proteolysis activity and the bacterial metabolism of the cells within the colony. However, the way the bacteria interact with dairy components is still poorly understood (Burgain et al., 2014). It is highly probable that, on one hand, milk proteins have to diffuse from the cheese matrix (a fat-protein network) into the colony to reach the bacterial cells in the center of the colony. Indeed, nutrients have to reach the center cells of the colony; otherwise these center cells may be starved. On the other hand, proteolysis end-products (small peptides and amino acids) have to diffuse from the bacterial colonies into the cheese matrix. If diffusion limitations occur inside the bacterial colony, gradients of concentration of both nitrogen sources (low concentrations in the center of the colony) and nitrogen end-products (high concentrations in the center of the colony) may be generated and may affect the metabolic activity of microbial cells, and thus the kinetics of the ripening process. The mean diameters of colonies and the mean distance between them in a model cheese were shown to be strongly influenced by the initial inoculation level. The lower the inoculation level was, the larger the colonies were, and then the further away they were from each other (Jeanson et al., 2011). It has previously been observed that low concentration of substrates could generate different physiological states or different growth rates in pathogenic bacterial colonies when colonies were bigger than $400 \mu \mathrm{m}$ diameter (McKay et al., 1997; Kreft et al., 1998). The main hypothesis for these observations was that the diffusion limitations of substrates within the bacterial colony lead to starvation for the cells in the center of the colony. As a consequence, lysis could be higher for the center cells in Vibrio cholera colonies (Wimpenny, 1992). If lysis occurs at the center of the colony, it is also very important to know if bacterial enzymes could diffuse out of the colony to determine how far from the colony proteolytic enzymes could diffuse outside the colony, in the cheese matrix.

However, Floury et al. (2010) reported a strong lack of data about the diffusion properties of key molecules like sugars, organic acids, proteins, and peptides in cheese. The first effective diffusion coefficient was determined for nisin in model cheeses (Aly et al., 2011). Using fluorescently labeled solutes, Silva et al. (2013) showed that dextran macromolecules up to $2 \mathrm{MDa}$, and different dairy proteins, were able to diffuse through the model cheese. Quite interestingly, the proteins tested (rigid and negatively charged molecules) were hindered to a greater degree than the dextrans (flexible and neutral molecules) in the model cheese, due to specific interactions between the protein matrix and the diffusing proteins. So far, only our previous study (Floury et al., 2013) has investigated the diffusion of molecules within bacterial colonies. Even if it has been demonstrated that viral particles such as bacteriophages $(\approx 100 \mathrm{~nm}$ head $)$ could diffuse inside biofilms (Lacroix-Gueu et al., 2005; Briandet et al., 2008), we consider that the structure of biofilms (with exopolysaccharide matrix) and colonies is not really comparable. It was then very important to understand ripening at the colony level by investigating the diffusion of model nutrient macromolecules, such as polysaccharides and milk proteins, inside lactococci colonies.

In our previous study (Floury et al., 2013), we developed a specific experimental design and we demonstrated for the first time that model solutes of different sizes (dextran macromolecules from 4.4 to $155 \mathrm{kDa}$ ) were able to diffuse inside bacterial colonies of $L$. lactis, immobilized in two different models of solid food matrices (model cheese and agar). The principle of this static design was to deposit a solution of the fluorescentlylabeled diffusing solute on the upper side of a gel cassette (Brocklehurst, 1995) filled with the solid medium, previously inoculated with $L$. lactis. The gel cassettes were then directly observed by confocal laser microscopy and the corresponding relative fluorescence intensity profiles within the colony vs. in the surrounding media were quantified after $3 \mathrm{~h}$ of diffusion of the fluorescent solutes at $19^{\circ} \mathrm{C}$. It was concluded that colonies of $L$. lactis LD61 immobilized in the model cheese were porous to all dextrans from 4 to $155 \mathrm{kDa}$ after this delay of migration, but no kinetic aspect of diffusion could be assessed.

The objective of the present work was to determine how diffusion phenomena inside colonies vary depending on both the properties of solutes and L. lactis strain. We quantified the diffusion rates of solutes of different sizes and physico-chemical properties both around and inside colonies immobilized in a model cheese, for two Lactococcus lactis strains presenting different surface properties. Our experimental device was improved by adapting the time-lapse microscopy method described in Rani et al. (2005), originally developed to determine the effective diffusion coefficients of fluorescent tracers into biofilm cell clusters of Staphylococcus epidermis and their surrounding solution.

\section{Materials and Methods}

\section{Bacterial Strains and Growth Conditions}

Lactococcus lactis subsp. lactis biovar diacetylactis LD61 was used (collection of the Centre International de Resources Microbiennes-Bactéries d'Intérêt Alimentaire (CIRM-BIA), INRA, Rennes, France) and was routinely grown under static conditions in M17 lactose broth (Difco, Becton Dickinson, Le Pont de Claix, France) at $30^{\circ} \mathrm{C}$.

Lactococcus lactis subsp. lactis TIL1230 was kindly given M-P Chapot-Chartier and obtained from the parental strain NCDO2110 (Giaouris et al., 2009). This strain was lactose and protease negative and was then grown under static conditions in M17 lactose broth supplemented with $0.5 \%$ glucose (Sigma) at $30^{\circ} \mathrm{C}$. Therefore, to ensure its optimal growth in milk, the milk 
cultures of TIL1230 were supplemented with $1 \%$ glucose (Sigma) and $0.3 \%$ peptone casein $(\mathrm{BD})$.

\section{Bacterial Surface Characterization Cell Surface Hydrophobicity}

Net surface charge of the bacteria and the presence of lipophilic compounds affect partitioning between two immiscible liquids (Burgain et al., 2014). The microbial adhesion to solvents (MATS) method was employed for the evaluation of the hydrophobic/hydrophilic character of the cell surface of L. lactis strains and for their Lewis acid-base characteristics. On this basis, we selected chloroform, a monopolar and acidic solvent (electron acceptor) and hexadecane, an apolar alkane using the protocol fully described in Giaouris et al. (2009). The values of MATS obtained with the chloroform were regarded as a measure of electron donor/basic characteristics of bacteria. Adhesion ability of the bacteria to the solvent is expressed as a percentage (\%) according to the following relation:

$$
\% \text { adhesion }=\frac{O D_{400}^{\text {initial aqueous phase }}-O D_{400}^{\text {aqueous phase after mixing }}}{O D_{400}^{\text {initial aqueous phase }}}
$$

With OD the optical density of the bacterial suspension measured at $400 \mathrm{~nm}$. Each measurement was performed in triplicate and the experiment was repeated twice with independent bacterial cultures.

\section{Cell Surface Charge}

The electrophoretic mobility (EM) was measured to determine the cell surface net charge of the two bacteria according to the protocol described in Boonaert and Rouxhet (2000). EM of the bacteria with the appropriate $\mathrm{pH}$ values were measured at room temperature on a Zetameter model (Zeta Sizer Nano Series, Malvern Instruments Ltd, Malvern, UK). Experiments were made twice with independent culture with triplicate measurements. EM was expressed in $10^{-8} \mathrm{~m}^{2} / \mathrm{V}$.s.

\section{Preparation of the Model Cheese in Imaging Chambers}

A fat-free cheese made from renneted concentrated skim milk was used as model cheese, as previously described in Floury et al. (2013). This non-fat model cheese has the great advantage over traditional cheese technology to be a repeatable and homogeneous cheese matrix. Moulded after renneting, it is coagulated without further syneresis of the gel, thus exhibiting highly reproducible micro- and macro- structural properties. The concentrated milk was inoculated for a final concentration of $10^{5} \mathrm{CFU} / \mathrm{ml}$, and coagulant agent (Maxiren 180; DSM Food Specialities, Seclin, France) was added at a final concentration of $300 \mu \mathrm{l} / \mathrm{l}$. After homogenization, $400 \mu \mathrm{l}$ of the mixture was slowly poured into several CoverWell imaging chambers (SigmaAldrich, Saint-Quentin Fallavier, France) that allow direct observation under the confocal microscope (Floury et al., 2012). The imaging chambers with the model cheese were then vertically incubated at $30^{\circ} \mathrm{C}$ for $15 \mathrm{~h}$ for coagulation and growth of the $L$. lactis LD61. For the L. lactis TIL1230, imaging chambers were also vertically incubated, for $8 \mathrm{~h}$ at $30^{\circ} \mathrm{C}$ and then for $15 \mathrm{~h}$ at $19^{\circ} \mathrm{C}$.
In parallel, the same media were also inoculated in $30 \mathrm{ml}$-bottles to measure the $\mathrm{pH}$ during acidification by L. lactis. The $\mathrm{pH}$ of the model cheeses were $5.05 \pm 0.06$, and $5.32 \pm 0.10$ for LD61 and TIL1230, respectively, after $15 \mathrm{~h}$ of incubation.

\section{Fluorescent Dyes and Labeled Solutes}

SYTO ${ }^{\mathrm{TM}}$ was added before coagulation of the model cheese to a final concentration of $1.2 \mu \mathrm{mol} / \mathrm{l}$, in order to dye the bacterial cells and to visualize colonies within the opaque matrix of cheese. SYTO9 ${ }^{\mathrm{TM}}$ penetrates all bacterial membranes and dies all the bacterial cells, alive, and damaged (Boulos et al., 1999).

Three Rhodamine B isothiocyanate (RITC) conjugated dextrans of 10,70 , and $155 \mathrm{kDa}$ were chosen as model of flexible and neutral polymers of anhydroglucose of different sizes (Table 1), labeled with an extent of labeling from 0.002 to 0.015 mol RITC per mol glucose (Sigma-Aldrich, Saint-Quentin Falavier, France). RITC-dextrans were dissolved to $50 \mathrm{mg} / \mathrm{ml}$ in distilled water.

The studied set of solutes was completed with three milk proteins, one random coil milk protein, the $\alpha_{S 1}$-casein (INRA, Rennes, France), and two globular dairy proteins, bovine serum albumin (BSA, Sigma-Aldrich, Saint-Quentin Falavier, France) and lactoferrin (LF, Fonterra Boulogne-Billancourt, France). The proteins were labeled with free RITC (Sigma) using the protocol described in Silva et al. (2013). The three solutions were lyophilized and labeling efficiencies were determined by mass spectroscopy. BSA, LF, and $\alpha_{S 1}$-casein were mainly mono-labeled with RITC. Finally, the RITC-labeled solutes were either dissolved to $50 \mathrm{mg} / \mathrm{ml}$ in water for the dextrans and the lactoferrin, in a permeate solution obtained from the ultrafiltration of skimmed milk for $\alpha_{S 1}$-casein, and in a $0.1 \mathrm{M}$ BisTris buffer at $\mathrm{pH} 6.8$ for the BSA.

The solutions of labeled solutes were stored at $-20^{\circ} \mathrm{C}$, protected from light before and during fluorescence measurements.

Physicochemical properties of the solutes are summarized in Table 1.

TABLE 1 | Physicochemical properties of the fluorescently-labeled solutes.

\begin{tabular}{|c|c|c|c|c|c|c|}
\hline & \multicolumn{3}{|c|}{ Dextran } & \multirow[t]{2}{*}{ BSA } & \multirow[t]{2}{*}{ Lactoferrin } & \multirow[t]{2}{*}{$\alpha_{\mathbf{S 1}}$-casein } \\
\hline & 10 & 70 & 155 & & & \\
\hline $\begin{array}{l}\text { Molecular } \\
\text { weight } \\
(\mathrm{kDa})\end{array}$ & 10 & 70 & 155 & $66.4^{a}$ & $77^{b}$ & $23.6^{c}$ \\
\hline $\begin{array}{l}\text { Isolectrical } \\
\text { point }\end{array}$ & - & - & - & $\approx 5^{a}$ & $8-9^{b}$ & $4.94^{d}$ \\
\hline $\begin{array}{l}\text { Hydrodynamic } \\
\text { radius }(\mathrm{nm})\end{array}$ & $2.3^{e}$ & $6^{e}$ & $8.5^{\mathrm{e}}$ & 3.65 & $2.2^{f}$ & $2.9^{\mathrm{C}}$ \\
\hline Flexibility & & flexible & & & rigid & flexible \\
\hline Hydrophobicity & & hydrophilic & & & drophobic & amphiphile \\
\hline
\end{tabular}




\section{Experimental Device for Solute Diffusion Experimental Set-up}

After the incubation time necessary for bacterial growth, a concentration gradient of the fluorescently-labeled solutes between the surface of the imaging chamber and the model cheese was triggered in order to induce the diffusion phenomenon. Five microliter of the fluorescently-labeled dextran or protein solution was dropped off at the surface of the coagulated model cheese and left to diffuse for $5 \mathrm{~min}$ throughout the surrounding medium, in the dark and in an air-conditioned room at $19^{\circ} \mathrm{C}$. Solute diffusion into the model cheeses began as soon as the fluorescent solution was left in contact with the surface of the cheese $(t=0)$. The fluorescent solutes diffuse into the gel by a plane one-dimensional diffusion mechanism (Figure 1).

\section{Time-Lapse Confocal Laser Scanning Microscopy}

Model cheese samples were imaged on an inverted NIKON Eclipse-TE2000-C1si microscope allowing confocal laser scanning microscopy (NIKON-France, Champigny sur Marne, France), with an oil-immersion $40 \times$ objective at $512 \times 512$ pixel resolution. Ten minutes after the deposit of the fluorescent solution containing the diffusing solutes, the first step was to localize a fluorescently (SYTO9 ${ }^{\mathrm{TM}}$ ) labeled colony, that had grown both in a focal plane at $10-15 \mu \mathrm{m}$ depth from the coverslip and at a quite close distance from the surface of the gel, in order to visualize the diffusion front of fluorescence of the RITC-labeled solutes in a reasonable time-scale. SYTO ${ }^{\mathrm{TM}}$ fluorescence was excited with the $488 \mathrm{~nm}$ laser and detected between 500 and $530 \mathrm{~nm}$. The second step was to obtain the kinetic of the diffusion process of the RITC labeled solutes thanks to the acquisition of images of the fluorescent front of diffusion around and into the target bacterial colony every 5-15 min for at least $2 \mathrm{~h}$. Fluorescently RITC-labeled dextrans and proteins were excited at $543 \mathrm{~nm}$ wavelength, and fluorescence emission was detected between 565 and $615 \mathrm{~nm}$. All experiments were performed at $19^{\circ} \mathrm{C}$ using a temperature-regulated platform and air-conditioned room and were performed at least in triplicate.

\section{Estimation of Diffusion Coefficients}

Images obtained after a time series of acquisition were analyzed using ImageJ software. A line measuring 512 pixels long and 10 pixels wide was drawn through the axial diameter of the colony in

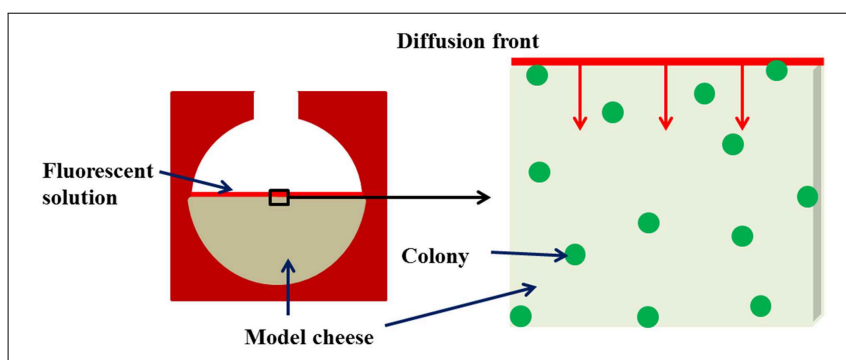

FIGURE 1 | Diagram of the experimental device for monitoring the kinetics of diffusion of solutes around and inside colonies immobilized in the model cheese. order to quantify the profile of RITC fluorescence intensity (also named gray-level value) along the direction of the diffusion front and at each acquisition time. The resulting fluorescence intensity profiles vs. time were then exported into a single spreadsheet.

According to Crank (1975), in the case of a plane one-dimensional diffusion, induced by an instantaneous source and after a short time $t$ of diffusion, the concentration gradient of the investigated solute is given by the following relationship:

$$
C(x, t)=\frac{M}{8 \sqrt{\pi D t}} \exp \left(-\frac{x^{2}}{4 D t}\right)
$$

where $C$ is the concentration expressed as the amount of diffusing solute per unit area of surface, $x$ is the perpendicular axis to the surface of the model cheese in $\mu \mathrm{m}$ ( $x=0$ corresponded to the surface), $t$ is the time, $D$ is the diffusion coefficient and $M$ is the surface concentration of the diffusing solute, which corresponds to the total amount of the investigated fluorescently labeled solute in the gel related to the gel surface unit. This concentration $M$ was considered as constant since it was in great excess compared to its concentration inside the cheese and as there was no reaction between the investigated component and the model cheese.

Equation (2) can be linearized:

$$
\operatorname{Ln}(C(x))=\operatorname{Ln}\left(\frac{M}{8 \sqrt{\pi D t}}\right)-\frac{x^{2}}{4 D t}
$$

The diffusion coefficient $D$ was estimated from the slope of the straight line $\operatorname{Ln}(C(x))$ vs. $x^{2}$, equal to $-1 /(4 D t)$. The slopes of the lines inside and outside the colonies were obtained by performing a linear regression with the best-fit linear trend function in Microsoft Excel using the least-squares method. Two effective diffusion coefficients $D_{\text {out }}$ and $D_{\text {in }}$ were quantified from the concentration profiles around the bacterial colony $\left(D_{\text {out }}\right)$ and inside the bacterial colony $\left(D_{\text {in }}\right)$. Both $D_{\text {out }}$ and $D_{\text {in }}$ were determined from the concentration profiles (gray values or fluorescence intensity profiles) obtained at a short time of diffusion, i.e., $30 \mathrm{~min}$ after the deposit, of the fluorescent solution at the surface of the model cheese.

\section{Statistical Analysis}

One-Way analysis of variance (ANOVA) and Tukey's paired comparison test were applied to the diffusion coefficient data in order to determine which mean values were significantly different from one another at the $95 \%$ confidence level using the R software package (version R i386 3.0.2).

\section{Results and Discussion}

\section{Surface of Both Lactoccoci Strains Are Hydrophilic, but TIL1230 is More Electronegative than LD61 at the pH of the Model Cheese}

The first step of the strategy of this study was to determine the hydrophobic/hydrophilic character, Lewis acid-base interactions, and electrostatic cell surface properties. The MATS method and EM measurements gave us information on the potential ability of 
TABLE 2 | Results of the microbial adhesion to solvents (MATS) method*.

\begin{tabular}{lrr}
\hline \multirow{2}{*}{ L. lactis strain } & \multicolumn{2}{c}{$\%$ of adhesion to } \\
\cline { 2 - 3 } & \multicolumn{1}{c}{ Hexadecane } & Chloroform \\
\hline LD61 & $8.6 \pm 5.2(n=4)$ & $11.3 \pm 9.8(n=3)$ \\
TIL1230 & $12.8 \pm 5.3(n=6)$ & $13.7 \pm 2.5(n=5)$ \\
\hline
\end{tabular}

${ }^{*}$ The results are expressed as the mean \pm one standard deviation of $n$ independent measurements.

the two L. lactis strains to generate physicochemical interactions between both the cheese matrix and the diffusing solutes.

Results of the MATS method are reported in Table 2.

The partitioning of cells between aqueous and hexadecane is a direct measurement of the cell surface hydrophobicity or hydrophilicity. The surface property of a cell can be considered as hydrophilic if its affinity for apolar hexadecane is below 40\% (Giaouris et al., 2009). As shown in Table 2, the percentage of adherent cells to hexadecane was slightly higher (not significantly) for TIL1230 than for LD61, with values largely inferior to $20 \%$ for both $L$. lactis strains, demonstrating a clear hydrophilic character of their surface. The hydrophilic character of bacteria is largely due to the nature of the compounds present on the surface, useful for adhesion (Burgain et al., 2014).

The percentages of bacterial adhesion to the chloroform, an acidic solvent and electron acceptor, were not significantly different between the two L. lactis strains, with values also inferior to $20 \%$ (Table 2). These results are in agreement with values of adhesion to chloroform obtained on various L. lactis strains by Ly et al. (2006) and Giaouris et al. (2009).

The electrophoretic mobilities (EM) of the two L. lactis strains at different $\mathrm{pH}$ values indicated that the isoelectric points were around $\mathrm{pH} 2.5$ and 4.5 for TIL1230 and LD61 strains, respectively (Figure 2). Between pH 2 and 6, the EM of L. lactis TIL1230 drastically decreased by about $4 \times 10^{-8}$, whereas it decreased only $0.4 \times 10^{-8} \mathrm{~m}^{2} /$ V.s for LD61. $L$. lactis TIL1230 has a greater EM above $\mathrm{pH} 3$ than LD61. Interestingly, LD61 strain presented EM very close to zero at all $\mathrm{pH}$ values tested, revealing very low electronegative cell surface in those conditions. In contrast, L. lactis TIL1230 was found to be highly negatively charged at $\mathrm{pH}$ between 4 and 6 , as previously observed for most of L. lactis strains, with same order of magnitude for EM values, ranging from -2 to $-5 \times 10^{-8} \mathrm{~m}^{2} / \mathrm{V} . \mathrm{s}$ (Ly et al., 2006; Habimana et al., 2007; Giaouris et al., 2009). Giaouris et al. (2009) reported for the first time that some lactic acid bacteria possess a very low surface electronegativity around neutral $\mathrm{pHs}$, as observed here for $L$. lactis LD61. This diversity in the global charge of lactococcal cell surface may be linked to the variability of the molecules containing ionized groups in the cell envelope. Three types of ionized groups are considered to determine the surface electrical properties of L. lactis: phosphate groups present in teichoic and lipoteichoic acids, and carboxylate and protonated amino groups of proteins (Boonaert and Rouxhet, 2000). It has been previously shown that the expression of the major cell wallanchored protease was responsible for altering L. lactis surface physicochemical properties, shifting the cell envelope from a

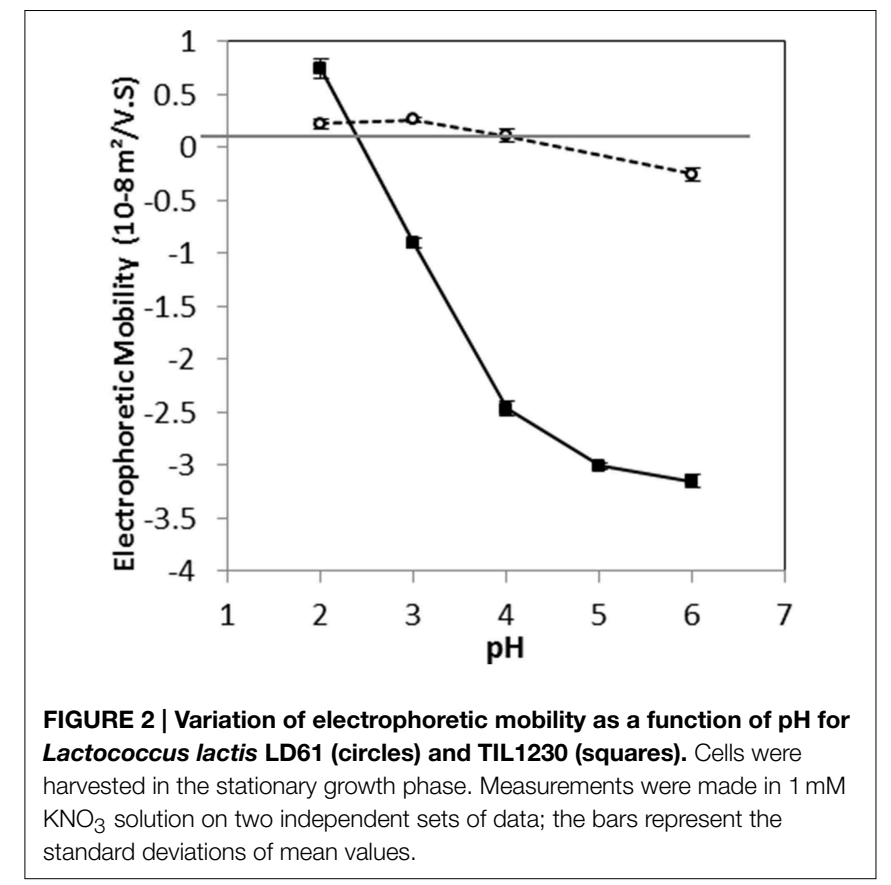

hydrophilic surface to an extremely hydrophobic one, going along with an increase of negative charges at the cell surface (Habimana et al., 2007). In the present study, the two strains of L. lactis LD61 and TIL1230 were thus supposed to present greater differences in their cell surface properties as TIL1230 does not possess the cell wall protease and LD61 does. Obviously, the global property of the cell surface is multi-causal and then difficult to predict.

Bacterial cell wall properties were shown to affect diffusion particles of nanoparticles inside biofilm matrices of L. lactis (Habimana et al., 2011). They measured that the diffusion of $50-\mathrm{nm}$ radius particles of anionic carboxylate-modified fluorescent polystyrene beads was more hindered in biofilm matrix of L. lactis which possessed the anchored protease. Based on these results, the present study aimed at comparing the diffusion of solutes of different charge, flexibility, and hydrophobicity, inside immobilized colonies (in cheese) of two different L. lactis strains presenting (LD61) or not (TIL1230) the anchored protease in their cell walls.

\section{Diffusion Coefficients of Dextrans Inside the Colonies Depend on the Solute Size but Not on the Lactococci Strains}

Figure 3A shows typical confocal microscopic observations of L. lactis colonies, immobilized in the model cheese, and visualized at different times after the deposit of the $70 \mathrm{kDa}$ fluorescently-labeled dextran solution. The images obtained with L. lactis TIL1230 and the two other fluorescently-labeled dextrans (10 and $155 \mathrm{kDa}$ ) were similar and are thus not shown here. Both lactococci strains grew in this model cheese as perfect spheres with diameters around $30-40 \mu \mathrm{m}$, as previously reported in Jeanson et al. (2011) and Floury et al. (2013). Figure $\mathbf{3 A}$ also clearly shows the progressive increase of the red 


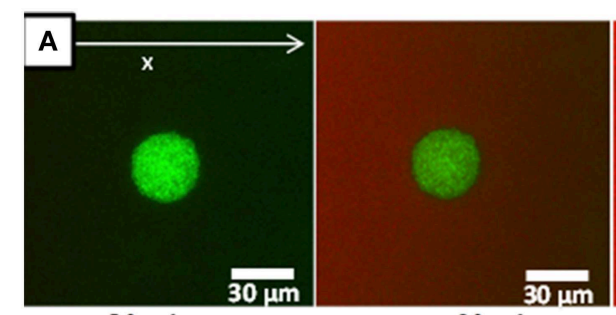

$10 \mathrm{~min}$
$30 \mathrm{~min}$

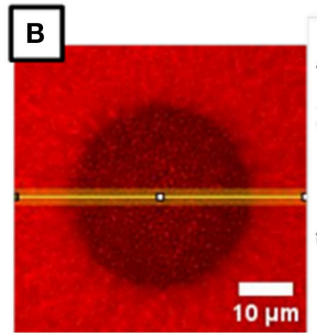

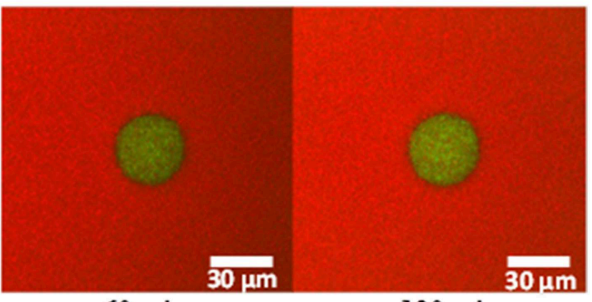

$60 \mathrm{~min}$

$120 \mathrm{~min}$

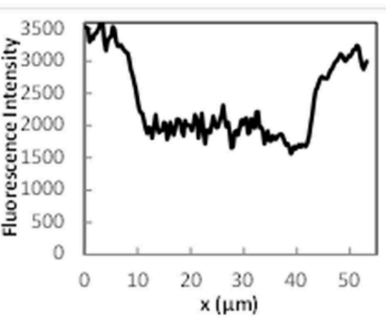

FIGURE 3 | (A) Example of a time-lapse microscopic observations of a Lactococcus lactis LD61 colony immobilized in a model cheese after $15 \mathrm{~h}$ growth at a temperature of $30^{\circ} \mathrm{C}$ during diffusion of a RITC-labeled dextran (here RITC-Dextran $70 \mathrm{kDa}$ ). L. lactis cells are colored in green, RITC-dextran in red. (B) Focus on the microscopic observation of the fluorescence intensity of RITC-dextran inside and around a $L$. lactis LD61 colony immobilized in a model cheese at $t=120$ min of diffusion and the corresponding fluorescence intensity profile along a 10 pixels wide line vs. position $x$. Black zones in the colony corresponds to the L. lactis cells. fluorescence both along the $\mathrm{x}$-axis and through time, proving that the fluorescent solute progressively moved inward toward a Lactococcus colony because of the concentration gradient between the surface and the interior of the model cheese. After $2 \mathrm{~h}$ of diffusion (Figure 3B), the red color was uniform in all the directions around the colony, meaning that the concentration of the fluorescently-labeled solute had reached a plateau. The diffusing process ended because there was no more concentration gradient in this area. The simple observation of these time series of images also suggests that the hypothesis of unidirectional diffusion of solute is valid. Therefore, image analysis of the intensity profiles of red fluorescence along this $\mathrm{x}$ axis allowed to directly quantifying the diffusive penetration of the solutes as a function of time (Figure 3B). Typical fluorescence intensity profiles were obtained (Figures 4 A-C) at different times (between 20 and $150 \mathrm{~min}$ ). Only an example of the fluorescence intensity profiles obtained with TIL1230 strain was shown on Figure 4D because the profiles were very similar to those obtained with LD61.

From all these fluorescence intensity data as a function of the position and the time of diffusion, we calculated the corresponding relative fluorescence intensity by dividing the fluorescence intensity at each position $\mathrm{x}$ by the average fluorescence intensity obtained on the region upstream the colony. An example is given on Figure $4 \mathrm{E}$ for the diffusion of the $70 \mathrm{kDa}$ dextran in a colony of L. lactis LD61. The relative fluorescence intensity profiles obtained with the other dextrans and with the other strain were very similar (data not shown). The purpose of this graphical representation of the results was to observe the evolution of the ratio of the fluorescence intensity in the colony vs. outside the colony as a function of time.
As shown on Figure 4E, whatever the time of diffusion considered, the fluorescence intensity inside the colony of both L. lactis strains drastically dropped compared to the fluorescence intensity in the surrounding cheese matrix, with a quite constant ratio around $0.4-0.6$ depending on the $\mathrm{x}$-axis position inside the colony. We previously showed that even the fluorescentlylabeled solutes diffuse inside a bacterial colony (Floury et al., 2013), but do not penetrate into the bacterial cells. Furthermore, dextrans are not metabolized by lactococci cells. Thus, the only way to explain this drop of relative fluorescence intensity inside the colony is because on the line of 10-pixel wide (Figure 3B), the volume filled with the fluorescent solution is lower inside the colony than outside the colony due to the presence of the bacterial cells (corresponding to black zones with no fluorescence intensity). So even if the experimental fluorescence intensity is different, the real concentration of the fluorescent solute is effectively the same both inside and around the colony. The calculated average value represented by the relative fluorescence intensity profiles was quite stable along the $\mathrm{X}$-axis inside the colonies for the fluorescently-labeled dextran solutes (Figure 3B), and this was also true whatever the time of diffusion considered (Figure 4E).

The concentration of the diffusing solute cannot be calculated from experimental data. The fluorescent intensity is, however, proportional to the diffusing solute concentration. Then, assuming a one-dimensional Fickian diffusion induced by an instantaneous source, the effective diffusion coefficients both inside the colony and in the surrounding matrix could be estimated from the slope of the linearization of the experimental fluorescence intensity profiles obtained a short time after the beginning of the diffusive process, with $D=-\frac{1}{4 . t . s l o p e}$ from Equation (3). A typical linearized curve and the corresponding 

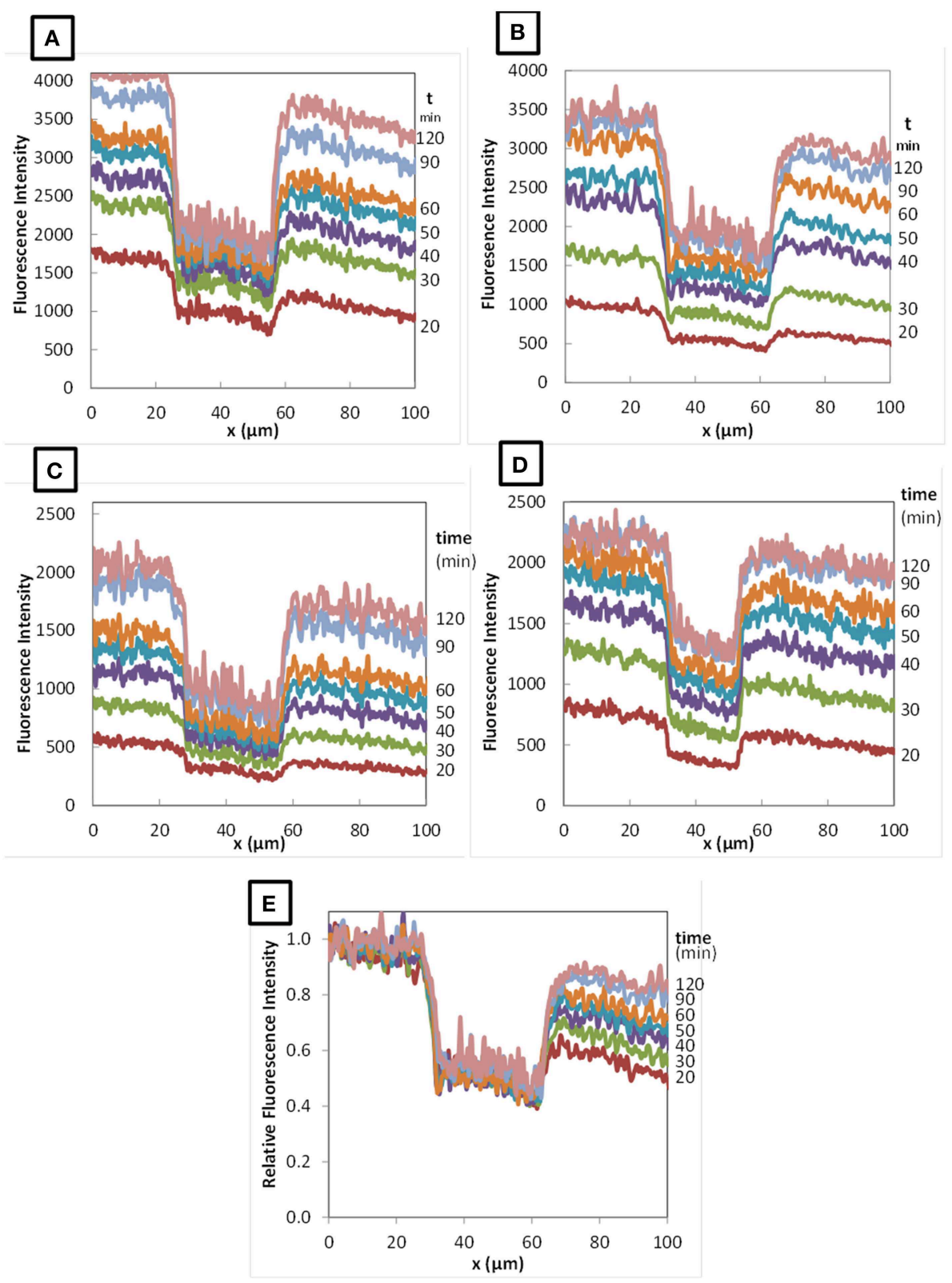

FIGURE 4 | Profiles of fluorescence intensity during diffusion of RITC-dextran $10 \mathrm{kDa}$ (A), $70 \mathrm{kDa}$ (B), and $155 \mathrm{kDa}$ (C) in colonies of Lactococcus lactis LD61 and of RITC-dextran $70 \mathrm{kDa}$ in
TIL1230 (D) as function of diffusion time (from 20 to $120 \mathrm{~min}$ ). (E) Typical relative fluorescence intensity profile obtained with RITC-dextran $70 \mathrm{kDa}$ in LD61. fitted equations obtained both outside and inside the colony are shown on Figure 5. Regression coefficients of the linear models were generally higher than 0.9 in the surrounding matrix, and slightly lower inside the colony with values around 0.7 because of a lower signal to noise ratio inside colonies. The slope of the line was clearly higher inside than outside the colony (Figure 5), suggesting that the diffusion of solutes was slower inside the colony. The diffusion of the fluorescent solute was probably more hindered inside the colony, most likely because of the high volume filled with the bacterial cells, than in the protein-network of the surrounding matrix.
The mean effective diffusion coefficients $\left(D_{\text {eff }}\right)$ of the different RITC-dextrans were obtained using this modeling approach, both inside and outside the colonies of the two bacterial strains (Figure 6). $D_{\text {eff }}$ were significantly $(p<0.05)$ lower inside the colonies than in the surrounding matrix. When $D_{\text {eff }}$ were plotted against the hydrodynamic radius, linear relationships $\left(R^{2}>0.8\right)$ were obtained over the molecular weight range of $10-155 \mathrm{kDa}$, both inside and outside colonies. The statistical analysis (ANOVA) performed on the estimated values revealed that the values of $D_{\text {eff }}$ were not significantly different $(p<0.001)$ between the two bacterial strains, meaning that dextran solutes 


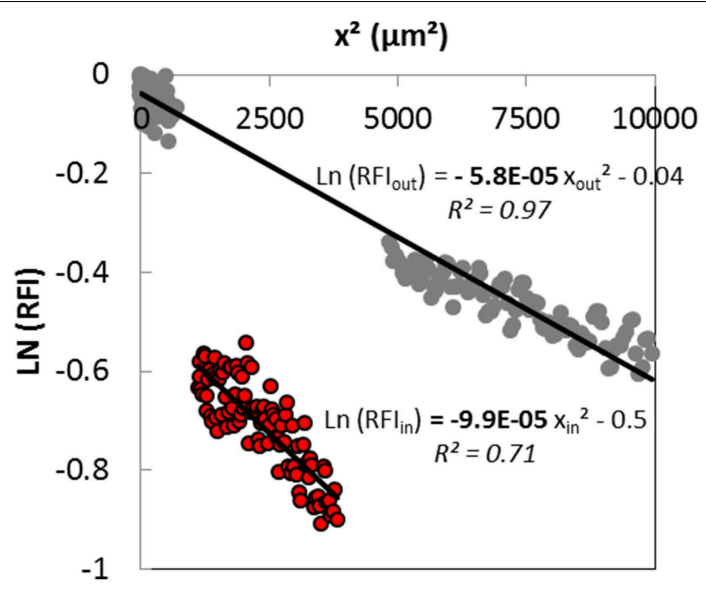

FIGURE 5 | Typical linearized curve obtained $30 \mathrm{~min}$ after the deposit of the fluorescent solution containing a $70 \mathrm{kDa}$ dextran at the surface of the model cheese inoculated with Lactococcus lactis LD61. Lines correspond to the linear fit of experimental data both outside (gray circles) and inside the colony (red circles).

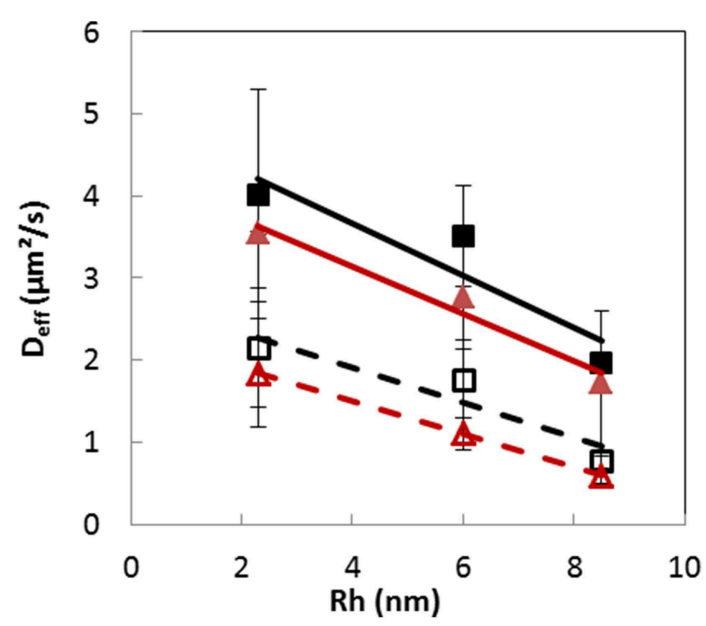

FIGURE 6 | Plots of the mean effective diffusion coefficients $\left(D_{\text {eff }}\right)$ of dextrans 10,70 , and $155 \mathrm{kDa}$ vs. their respective hydrodynamic radius, estimated from experimental data obtained after $30 \mathrm{~min}$, both inside (open symbols) and outside (plain symbols) colonies of Lactoccus lactis LD61 (squares) and TIL1230 (triangles) in a model cheese. Lines, linear regression.

diffused inside both Lactocci colonies with a similar diffusion coefficient, which depended mainly on size of the solute. The absence of significant difference between both $L$. lactis strains was expected because dextrans are known to be hydrophilic macromolecules and the surface properties of the two different cells were shown to be also both very hydrophilic.

In the present study, we went further than the previous study (Floury et al., 2013) by visualizing the kinetic of the diffusion process inside the colonies. Moreover, we were able to estimate diffusion coefficients of the dextran solutes inside the microbial colonies from image analysis of the data. As shown in Table 3, whatever their size between 10 and $155 \mathrm{kDa}$ and the Lactoccocus
TABLE 3 | Calculated ratios of effective diffusion coefficients of dextrans obtained inside and outside $L$. lactis colonies $\left(D_{\text {in }} / D_{\text {out }}\right)$, of diffusion coefficients of dextrans in water* vs. inside colonies $\left(D_{\mathrm{w}} / D_{\text {in }}\right)$, and corresponding tortuosity indices, calculated as square root of $D_{\mathrm{w}} / D_{\mathrm{in}}$.

\begin{tabular}{lcccccc}
\hline $\begin{array}{l}\text { Dextran } \\
\text { MW (kDa) }\end{array}$ & \multicolumn{2}{c}{$\boldsymbol{D}_{\text {in }} / \boldsymbol{D}_{\text {out }}$} & \multicolumn{2}{c}{$\boldsymbol{D}_{\mathbf{w}}{ }^{*} / \boldsymbol{D}_{\text {in }}$} & \multicolumn{2}{c}{ Tortuosity index } \\
\cline { 2 - 7 } & \multicolumn{5}{c}{ L. lactis strain } \\
\cline { 2 - 7 } & LD61 & TIL1230 & LD61 & TIL1230 & LD61 & TIL1230 \\
\hline 10 & 0.54 & 0.52 & 43 & 50 & 6.6 & 7.1 \\
70 & 0.50 & 0.40 & 20 & 32 & 4.5 & 5.7 \\
155 & 0.40 & 0.34 & 32 & 43 & 5.7 & 6.5 \\
\hline
\end{tabular}

${ }^{*} D w$ values at $20^{\circ} \mathrm{C}$ calculated by using the Stokes-Einstein relationship.

strain, the effective diffusion coefficients of dextrans obtained inside the colonies were around twice lower than their respective diffusion coefficients in the surrounding cheese matrix around the colonies, and up to 50 times smaller than those in water $D_{\mathrm{w}}$ (calculated from the Stokes-Einstein equation). Silva et al. (2013) also found that effective diffusion coefficient values of fluorescein isothiocyanate (FITC)-dextrans (from 4 to $2000 \mathrm{kDa}$ ) in the same model cheese, but not inoculated, were smaller than those in water due to the hindrance of the protein network. However, their values of effective diffusion coefficients were between 4 and 9 times larger than in the present study, depending of the size of the dextran.

The variability observed has two major causes. The first source of variability is the experimental approaches used to estimate diffusion coefficients (Floury et al., 2010). Effective diffusion coefficients estimated thanks to the concentration profiles method in the present method are then difficult to compared to the so called "self-diffusion" coefficients obtained using the FRAP technique in Silva et al. (2013).

The second source of variability is the model cheese. Indeed, even if the model cheese had the same initial composition in both studies, the metabolism of the lactococci inoculated in the present study induced a strong decrease of cheese $\mathrm{pH}$ from 6.6 to 5.1-5.3, depending on the L. lactis strain, which probably modified to some extent the microstructure of the protein network and then the diffusion behavior of the solutes in both cheeses.

In agreement with our results, Guiot et al. (2002) and Thurnheer et al. (2003) observed that the diffusion coefficients of fluorescently-labeled dextrans from 3 to $70 \mathrm{kDa}$ also decreased linearly with hydrodynamic radius in different mono- and polyspecies biofilms, and were up to 150 times smaller than those in bulk water. However, the direct comparison of their results with our study is difficult because the microbial cell distribution is rather different in a biofilm and in a food matrix such as cheese. To our knowledge, only two studies are realistically comparable to our system (Rani et al., 2005 and Takenaka et al., 2009). They focused the analysis of the solute diffusion exclusively within identified clusters of microbial cells inside different model oral biofilms. The diffusive penetration of two tracer molecules (rhodamine B and fluorescein, $\mathrm{MW} \sim 400 \mathrm{Da}$, chosen as model of antibiotic for their similar size), into staphylococcal cell clusters was directly visualized by confocal 
scanning laser microscopy (Rani et al., 2005). The effective diffusion coefficients of the two fluorescent tracers were around 10 times lower than the corresponding solute diffusion coefficient in water. The difference with the present study could be due to a denser population of cells in our colonies. The diffusive penetration of fluorescently-labeled dextrans of various molecular weights (from 3 to $70 \mathrm{kDa}$ ) was visualized into three different species of cell clusters formed by oral bacteria grown in a flow cell (Takenaka et al., 2009). Like in the present study, the effective diffusion coefficient of dextrans strongly decreased with their molecular weights. However, their order of magnitude was different from our results, with effective diffusion coefficients only twice smaller than those in water. For Thurnheer et al. (2003), analysis of diffusion phenomena within biofilms suggested tortuosity as the most probable factor responsible for retarded diffusion compared to water. They defined a tortuosity index, as the square root of $D_{\mathrm{w}} / D_{\text {in }}$, representing an indicator of solute diffusion through interstitial space between bacterial cells. A molecule going through a highly convoluted threedimensional route in a matrix will be delayed in comparison with free diffusion in water. As shown in Table 3, the tortuosity indexes estimated from our experimental data were of the same order of magnitude regardless of the L. lactis strain and the size of the diffusing dextran solutes. In our conditions, the extracellular space between the lactococci cells within the colony is filled with an aqueous phase composed of water, lactose, and minerals (Floury et al., 2013), thus explaining why the tortuosity index did not depend on the size of the dextrans.

In conclusion about the diffusion of dextrans within bacterial colony, we confirmed that macromolecules as large as dextrans of $155 \mathrm{kDa}$ diffused into lactococci colonies in a model cheese (Floury et al., 2013). We demonstrated that their diffusion was similar for two different strains of lactoccoci whatever the size of dextrans up to $155 \mathrm{kDa}$.

\section{Milk Proteins, Such as BSA, Lactoferrin and $\alpha_{S 1}$-Casein, Do Not Diffuse Inside neither LD61 nor TIL1230 Lactococci Colonies}

The typical fluorescence intensity profiles for three fluorescentlylabeled proteins, and typical images of the corresponding colonies at the end of the experiments are shown on Figures 7-9. Whereas, the fluorescence intensity of the three proteins outside the colonies increased with time, the fluorescence intensity measured inside the colonies was very low all along the duration of the experiments. The increase of the fluorescence intensity throughout time outside the colonies shows that the three

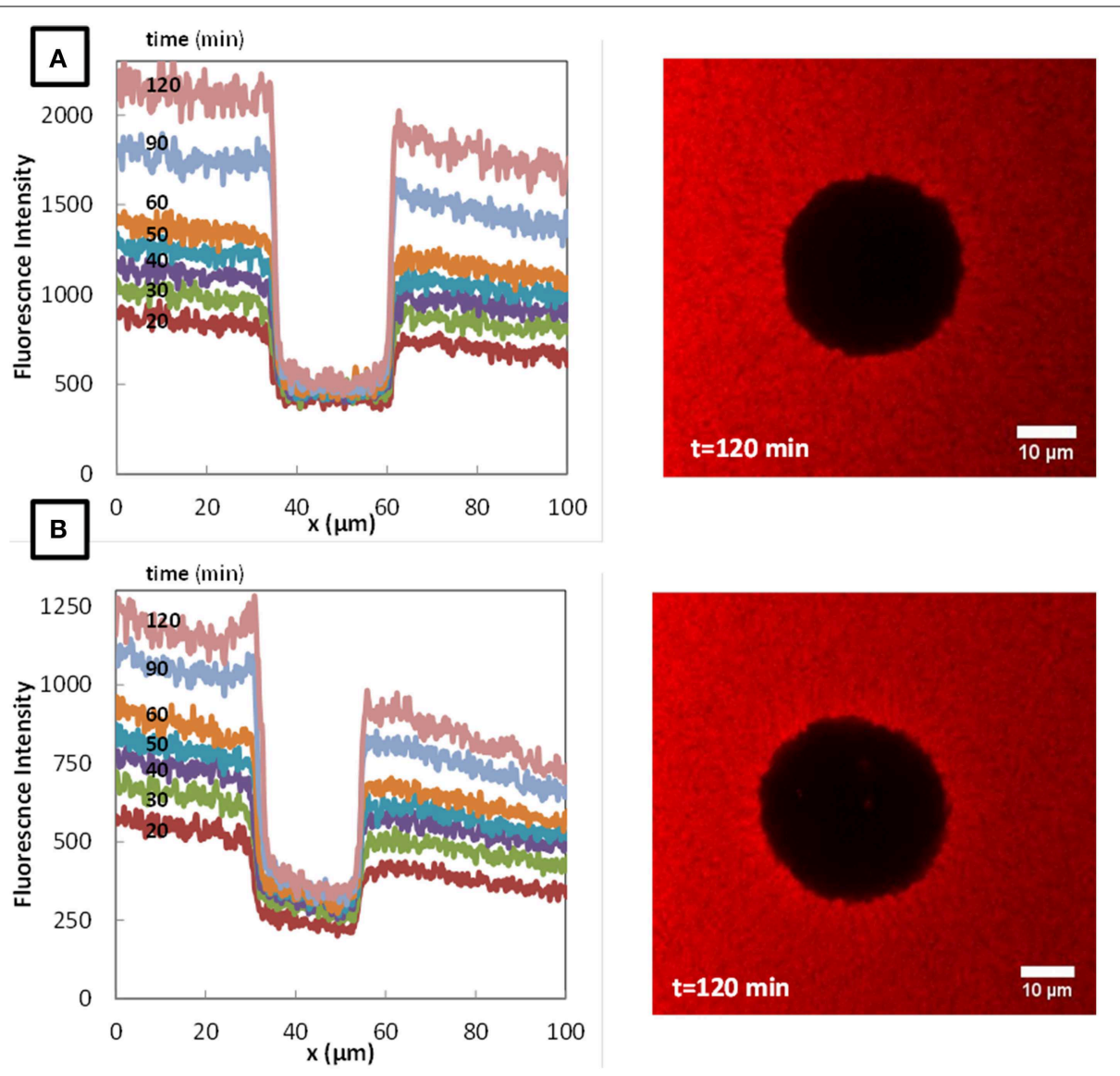

FIGURE 7 | Fluorescence profiles of fluorescently-labeled Bovine Serum Albumin (BSA), in colonies of Lactococcus lactis LD61 (A) and TIL 1230 (B) at different times from 20 to $120 \mathrm{~min}$ after the deposit at the surface of a model cheese and the corresponding microscopic observations of the colony after $120 \mathrm{~min}$ of diffusion. 

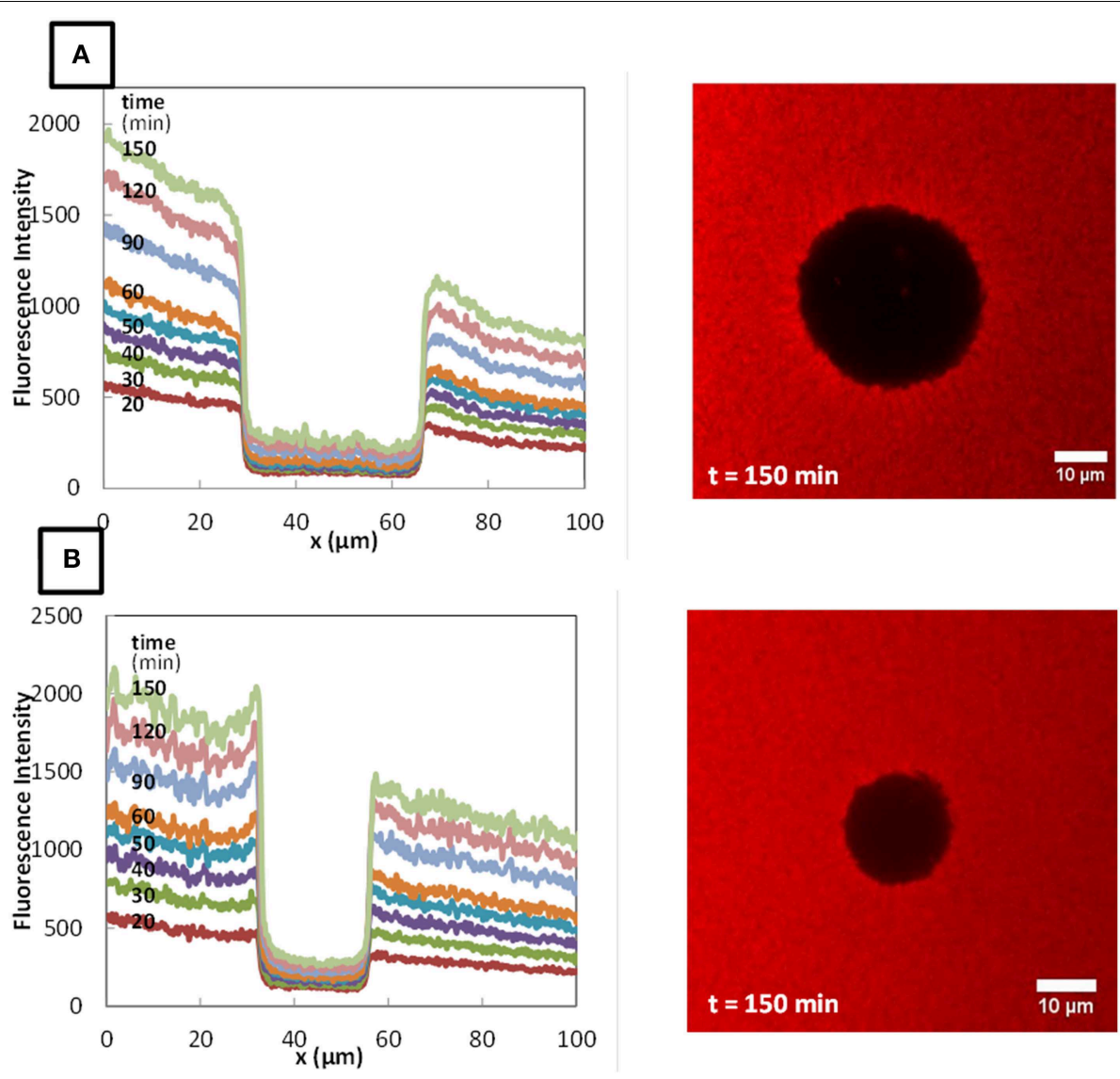

FIGURE 8 | Fluorescence profiles of RITC-LF in Lactococcus lactis LD61 (A) and TIL1230 (B) from 20 to 150 min and corresponding microscopic observations of the colony after $150 \mathrm{~min}$ of diffusion.

labeled-proteins effectively diffused in the surrounding cheese matrix. Whereas, the fluorescence outside the colonies was finally intense, the observation of the micrographs clearly confirmed the intensity profiles by the absence of fluorescence inside the colonies. This means that, surprisingly, none of the three proteins could diffuse inside the bacterial colonies, although their hydrodynamic radii were much smaller than the radius of the largest dextran (Table 1). Concerning the diffusion of proteins in cell clusters, to our knowledge the only published study is from Takenaka et al. (2009).Contrary to our results, they clearly visualized by time-lapse confocal microscopic observations that fluorescently-labeled proteins, even the largest like ConA (MW $104 \mathrm{kDa}$ ) and IgG (MW $150 \mathrm{kDa})$, diffused inside microbial cell clusters that were approximately a few hundred micrometers in diameter and reached the center of these cell clusters in less than $3 \mathrm{~min}$.

These results highlighted that the size of the diffusing solute was not the sole factor conditioning its ability to enter inside colonies of $L$. lactis immobilized in cheese. Other physicochemical factors such as flexibility, charge and/or hydrophobicity of the solute can also be of involved, by generating bacteria-solute interactions of different nature, especially for diffusing solutes such as milk proteins. Moreover, the impact of these factors can also depend on the surface properties of the strain (Habimana et al., 2011).
When diffusing through a non-inoculated model cheese, the rigid, and globally negatively charged BSA protein was hindered more than dextrans with a similar hydrodynamic radius, because of the existence of solute-matrix interactions (Silva et al., 2013). In the same way, deBeer et al. (1997) and Takenaka et al. (2009) reported that diffusion coefficients of solutes in different kinds of cell clusters were conditioned on the network structure in the interstitial space, but also mainly depended on the size and the charge of the diffusing solute. Unlike dextrans that are flexible, neutral, and hydrophilic polymers, proteins possess different shapes, hydrophobicity, and charges (Table 1). Moreover, even if the surface of the cells of the two L. lactis strains were shown to be both hydrophilic, L. lactis TIL1230 was more electronegative than LD61 at the $\mathrm{pH}$ of the model cheeses. Therefore, we could have expected the surface properties of bacterial cells to influence the ability of solutes to diffuse or not inside the colony by generating either repulsive or attractive interactions like electrostatic forces, depending on the charge of the solute (Burgain et al., 2014). The $\mathrm{pH}$ of the model cheeses were around 5.1 and 5.3 for LD61 and TIL1230, respectively. Therefore, according to the isoelectric point of the proteins (Table 1), the net charges of BSA and $\alpha$-s1 casein were slightly negative, whereas LF was globally positively charged in both cheese matrices. Electrostatic repulsions could have then occurred between the outer bacterial cells of the colony and the negatively charged solutes, preventing their diffusion 


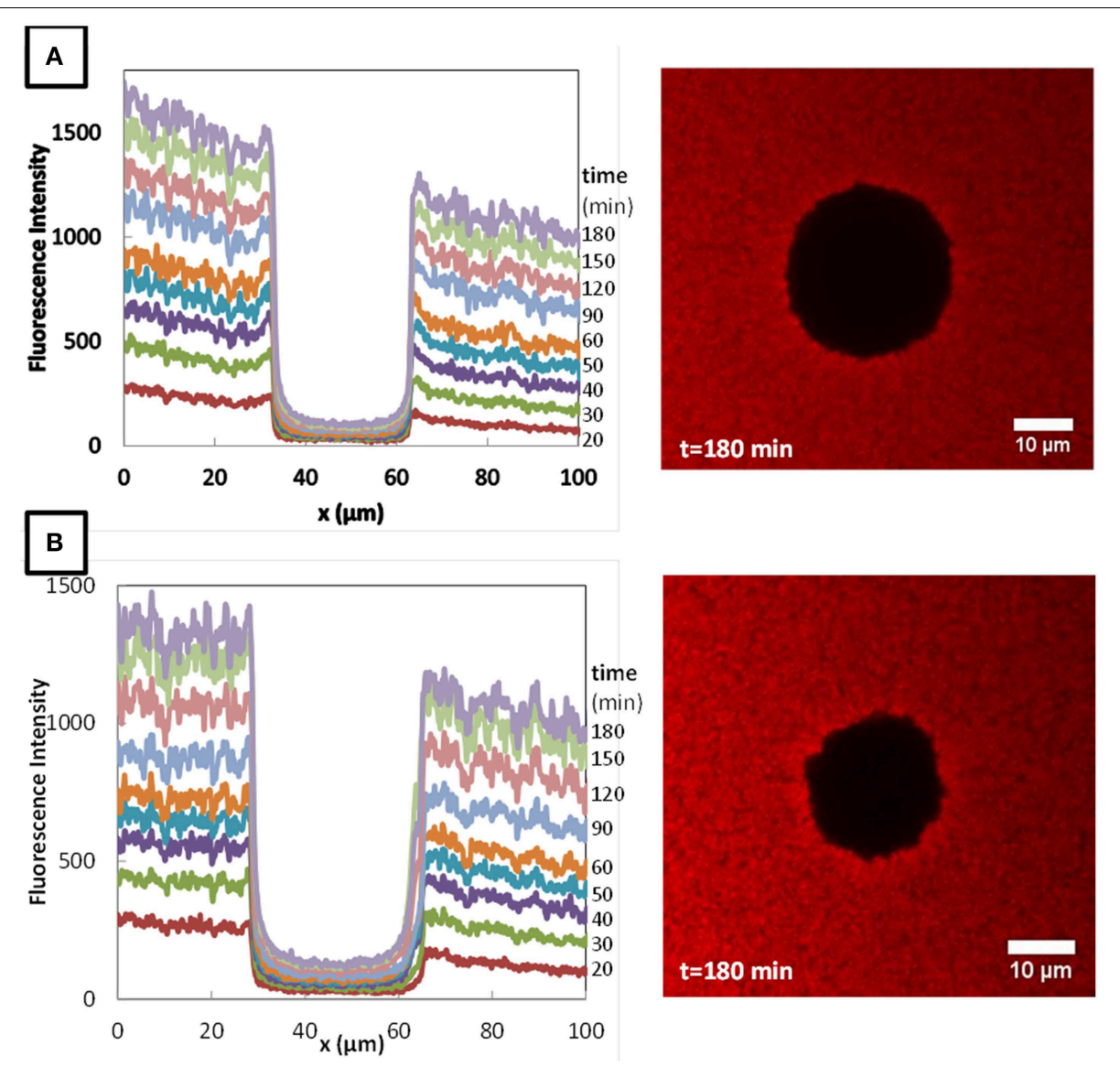

FIGURE 9 | Fluorescence profiles of RITC- $\alpha_{\mathrm{S} 1}$-casein in Lactococcus lactis LD61 (A) and TIL1230 (B) from 20 to 180 min and corresponding microscopic observations of the colony after $180 \mathrm{~min}$ of diffusion.

inside colony. However, it is largely known that cheese is a medium presenting a high ionic strength (around $100 \mathrm{mM}$ ). In that case, the Debye length is very small (around $2 \mathrm{~nm}$ ) and then the energy barrier due to repulsive forces is very low, meaning that the electrostatic contribution is strongly suppressed (Burgain et al., 2011). Interactions of electrostatic nature were not explaining the non-ability of the three milk proteins to penetrate inside L. lactis colonies immobilized in cheese. Other kind of repulsive forces such as hydrophobic interactions can be involved, especially between the hydrophobic BSA and LF proteins and the hydrophilic surfaces of the cells of both L. lactis strains. However, for the amphiphilic $\alpha$-s1 casein, there was no reason for repulsions of hydrophobic origin with the bacterial surfaces.

We were finally not able to explain why $\alpha$-s1 casein proteins could not diffuse inside the lactococci colonies. However, it is well-known that milk caseins, especially the $\alpha$-s1 casein, are hydrolyzed by the cell wall proteases of $L$. lactis in cheese and/or other proteases present in the cheese. We can thus hypothesize that some of the generated peptides can diffuse inside colonies, and are further metabolized into smaller peptides and amino acids by all the cells located inside the colonies, as strongly suggested by the results obtained in the same model cheese by Le Boucher et al. (personal communication).

\section{Conclusion}

Effective diffusion coefficients of dextran macromolecules were quantified for the first time inside colonies of two different $L$. lactis strains immobilized in a model cheese. We clearly showed that the diffusion behavior of macromolecules through bacterial colonies immobilized in a model cheese not only depends on the size of the diffusing solutes, but also and mainly on their physicochemical properties. Whereas, a flexible and neutral hydrophilic polymer such as a dextran can diffuse inside colonies whatever its size, none of the three proteins investigated in this study could penetrate inside lactococci colonies. These original results remain unexplained because both the surface of the two bacterial strains and the three diffusing proteins presented various physicochemical properties, from rigid to flexible shapes, and from negatively to neutral and positively charged. Our results finally show that the choice of the fluorescently-labeled molecule as a model of diffusing solute is crucial.

\section{Acknowledgments}

This study was part of the CheeseOmic Project co-funded by the regions Bretagne and Pays-de-la-Loire and supported by Bretagne Biotechnologie Alimentaire (BBA) association. 


\section{References}

Aly, S., Floury, J., Famelart, M. H., Madec, M. N., Dupont, D., Le Gouar, Y., et al. (2011). Nisin quantification by ELISA allows the modeling of its apparent diffusion coefficient in model cheeses. J. Agric. Food Chem. 59, 9484-9490. doi: $10.1021 /$ jf2008474

Böhme, U., and Scheler, U. (2007). Effective charge of bovine serum albumin determined by electrophoresis NMR. Chem. Phys. Lett. 435, 342-345. doi: 10.1016/j.cplett.2006.12.068

Bokkhim, H., Bansal, N., GrØndahl, L., and Bhandari, B. (2013). Physico-chemical properties of different forms of bovine lactoferrin. Food Chem. 141, 3007-3013. doi: 10.1016/j.foodchem.2013.05.139

Boonaert, C. J., and Rouxhet, P. G. (2000). Surface of lactic acid bacteria: relationships between chemical composition and physicochemical properties. Appl. Environ. Microbiol. 66, 2548-2554. doi: 10.1128/AEM.66.6.25482554.2000

Boulos, L., Prévost, M., Barbeau, B., Coallier, J., and Desjardins, R. (1999). LIVE/DEAD ${ }^{\circledR}$ BacLight ${ }^{\mathrm{TM}}$ : application of a new rapid staining method for direct enumeration of viable and total bacteria in drinking water. J. Microbiol. Methods 37, 77-86. doi: 10.1016/S0167-7012(99)00048-2

Briandet, R., Lacroix-Gueu, P., Renault, M., Lecart, S., Meylheuc, T., Bidnenko, E., et al. (2008). Fluorescence correlation spectroscopy to study diffusion and reaction of bacteriophages inside biofilms. Appl. Environ. Microbiol. 74, 2135-2143. doi: 10.1128/AEM.02304-07

Brocklehurst, T. (1995). The effect of transient temperatures on the growth of Salmonella typhimurium LT2 in gelatin gel. Int. J. Food Microbiol. 27, 45-60. doi: 10.1016/0168-1605(94)00152-V

Burgain, J., Gaiani, C., Linder, M., and Scher, J. (2011). Encapsulation of probiotic living cells: from laboratory scale to industrial applications. J. Food Eng. 104, 467-483. doi: 10.1016/j.jfoodeng.2010.12.031

Burgain, J., Scher, J., Lebeer, S., Vanderleyden, J., Cailliez-Grimal, C., Corgneau, M., et al. (2014). Significance of bacterial surface molecules interactions with milk proteins to enhance microencapsulation of Lactobacillus rhamnosus GG. Food Hydrocoll. 41, 60-70. doi: 10.1016/j.foodhyd.2014.03.029

Chaufer, B., Rabiller-Baudry, M., Lucas, D., Michel, F., and Timmer, M. (2000). Selective extraction of lysozyme from a mixture with lactoferrin by ultrafiltration. Role of the physico-chemical environment. Le Lait 80, 197-203. doi: 10.1051/lait:2000119

Crank, J. (1975). The Mathematics of Diffusion, 2nd Edn. Oxford: Clarendon Press.

deBeer, D., Stoodley, P., and Lewandowski, Z. (1997). Measurement of local diffusion coefficients in biofilms by microinjection and confocal microscopy. Biotechnol. Bioeng. 53, 151-158.

Floury, J., Jeanson, S., Aly, S., and Lortal, S. (2010). Determination of the diffusion coefficients of small solutes in cheese: a review. Dairy Sci. Technol. 90, 477-508. doi: $10.1051 / \mathrm{dst} / 2010011$

Floury, J., Jeanson, S., Madec, M.-N., and Lortal, S. (2013). Porosity of Lactococcus lactis subsp. lactis LD61 colonies immobilised in model cheese. Int. J. Food Microbiol. 163, 64-70. doi: 10.1016/j.ijfoodmicro.2013.02.014

Floury, J. M. N., Madec, F., Waharte, S., Jeanson, S., and, S., Lortal (2012). First assessment of diffusion coefficients in model cheese by fluorescence recovery after photobleaching (FRAP). Food Chem. 133, 551-556. doi: 10.1016/j.foodchem.2012.01.030

Giaouris, E., Chapot-Chartier, M.-P., and Briandet, R. (2009). Surface physicochemical analysis of natural Lactococcus lactis strains reveals the existence of hydrophobic and low charged strains with altered adhesive properties. Int. J. Food Microbiol. 131, 2-9. doi: 10.1016/j.ijfoodmicro.2008.09.006

Guiot, E., Georges, P., Brun, A., Fontaine-Aupart, M. P., Bellon-Fontaine, M. N., and Briandet, R. (2002). Heterogeneity of diffusion inside microbial biofilms determined by fluorescence correlation spectroscopy under two-photon excitation. Photochem. Photobiol. 75, 570-578. doi: 10.1562/0031-8655(2002)075<0570:HODIMB > 2.0.CO;2

Habimana, O., Le Goff, C., Juillard, V., Bellon-Fontaine, M.-N., Buist, G., Kulakauskas, S., et al. (2007). Positive role of cell wall anchored proteinase PrtP in adhesion of lactococci. BMC Microbiol. 7:36. doi: 10.1186/1471-2180-7-36

Habimana, O., Steenkeste, K., Fontaine-Aupart, M.-P., Bellon-Fontaine, M.-N., Kulakauskas, S., and Briandet, R. (2011). Diffusion of nanoparticles in biofilms is altered by bacterial cell wall hydrophobicity. Appl. Environ. Microbiol. 77, 367-368. doi: 10.1128/AEM.02163-10

Jeanson, S., Chad uf, J., Madec, M. N., Aly, S., Floury, J., Brocklehurst, T. F., et al. (2011). Spatial distribution of bacterial colonies in a model cheese. Appl. Environ. Microbiol. 77, 1493-1500. doi: 10.1128/AEM.02233-10

Kreft, J. U., Booth, G., and Wimpenny, J. W. (1998). BacSim, a simulator for individual-based modelling of bacterial colony growth. Microbiol. Read. Engl. 144(Pt. 12), 3275-3287. doi: 10.1099/00221287-144-12-3275

Lacroix-Gueu, P., Briandet, R., Leveque-Fort, S., Bellon-Fontaine, M. N., and Fontaine-Aupart, M. P. (2005). In situ measurements of viral particles diffusion inside mucoid biofilms. C. R. Biol. 328, 1065-1072. doi: 10.1016/j.crvi.2005.09.010

Ly, M. H., Naïtali-Bouchez, M., Meylheuc, T., Bellon-Fontaine, M.-N., Le, T. M., Belin, J.-M., et al. (2006). Importance of bacterial surface properties to control the stability of emulsions. Int. J. Food Microbiol. 112, 26-34. doi: 10.1016/j.ijfoodmicro.2006.05.022

Marchin, S., Putaux, J.-L., Pignon, F., and Léonil, J. (2007). Effects of the environmental factors on the casein micelle structure studied by cryo transmission electron microscopy and small-angle $\mathrm{x}$-ray scattering/ultrasmallangle X-ray scattering. J. Chem. Phys. 126, 045101. doi: 10.1063/1.2409933

McKay, A. L., Peters, A. C., and Wimpenny, J. W. T. (1997). Determining specific growth rates in different regions of Salmonella typhimurium colonies. Lett. Appl. Microbiol. 24, 74-76. doi: 10.1046/j.1472-765X.1997.00354.x

Rani, S. A., Pitts, B., and Stewart, P. S. (2005). Rapid diffusion of fluorescent tracers into Staphylococcus epidermidis biofilms visualized by time lapse microscopy. Antimicrob. Agents Chemother. 49, 728-732. doi: 10.1128/AAC.49.2.728732.2005

Silva, J. V. C., Peixoto, P. D. S., Lortal, S., and Floury, J. (2013). Transport phenomena in a model cheese: the influence of the charge and shape of solutes on diffusion. J. Dairy Sci. 96, 6186-6198. doi: 10.3168/jds.2013-6552

Takenaka, S., Pitts, B., Trivedi, H. M., and Stewart, P. S. (2009). Diffusion of macromolecules in model oral biofilms. Appl. Environ. Microbiol. 75, 1750-1753. doi: 10.1128/AEM.02279-08

Thurnheer, T., Gmur, R., Shapiro, S., and Guggenheim, B. (2003). Mass transport of macromolecules within an in vitro model of supragingival plaque. Appl. Environ. Microbiol. 69, 1702-1709. doi: 10.1128/AEM.69.3.17021709.2003

Wimpenny, J. W. T. (1992). "Microbial systems," in Advances in Microbial Ecology, ed K. C. Marshall (Boston, MA: Springer US), 469-522. Available online at: http://link.springer.com/10.1007/978-1-4684-7609-5_10 (Accessed February 2, 2015).

Conflict of Interest Statement: The authors declare that the research was conducted in the absence of any commercial or financial relationships that could be construed as a potential conflict of interest.

Copyright (C) 2015 Floury, El Mourdi, Silva, Lortal, Thierry and Jeanson. This is an open-access article distributed under the terms of the Creative Commons Attribution License (CC BY). The use, distribution or reproduction in other forums is permitted, provided the original author(s) or licensor are credited and that the original publication in this journal is cited, in accordance with accepted academic practice. No use, distribution or reproduction is permitted which does not comply with these terms. 


\section{ADVANTAGES OF PUBLISHING IN FRONTIERS}
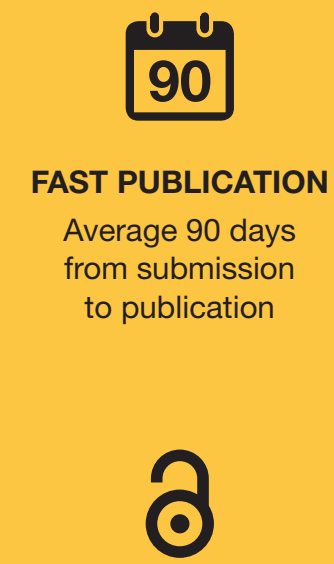

OPEN ACCESS

Articles are free to read, for greatest visibility

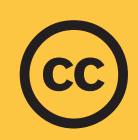

\section{COPYRIGHT TO AUTHORS}

No limit to article distribution and re-use

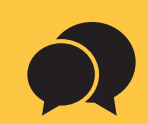

COLLABORATIVE

PEER-REVIEW

Designed to be rigorous yet also collaborative, fair and constructive

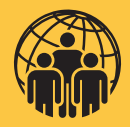

TRANSPARENT

Editors and reviewers acknowledged by name on published articles

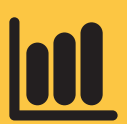

IMPACT METRICS

Advanced metrics track your article's impact

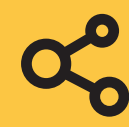

\section{RESEARCH NETWORK}

Our network increases readership

for your article

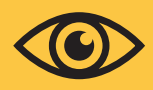

\section{GLOBAL SPREAD}

Six million monthly page views worldwide

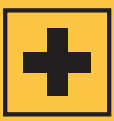

SUPPORT

By our Swiss-based editorial team 